\title{
Investigating Geometric Uncertainties in Prostate Cancer Radiotherapy
}

\author{
by \\ Zhanrong Gao \\ Master of Science (Carleton University, 2001) \\ A thesis submitted to the \\ Faculty of Graduate Studies and Research \\ in partial fulfilment of \\ the degree requirements of \\ Doctor of Philosophy \\ Ottawa-Carleton Institute for Physics \\ Department of Physics \\ Carleton University \\ Ottawa, Ontario, Canada \\ March 2006 \\ Copyright $(2006$ - Zhanrong Gao
}




$\begin{array}{ll}\begin{array}{l}\text { Library and } \\ \text { Archives Canada }\end{array} & \begin{array}{l}\text { Bibliothèque et } \\ \text { Archives Canada }\end{array} \\ \begin{array}{l}\text { Published Heritage } \\ \text { Branch }\end{array} & \begin{array}{l}\text { Direction du } \\ \text { Patrimoine de l'édition }\end{array} \\ \begin{array}{l}\text { 395 Wellington Street } \\ \text { Ottawa ON K1A ON4 }\end{array} & \begin{array}{l}\text { 395, rue Wellington } \\ \text { Ottawa ON K1A ON4 } \\ \text { Canada }\end{array}\end{array}$

Your file Votre référence ISBN: 978-0-494-18219-2 Our file Notre référence ISBN: 978-0-494-18219-2

NOTICE:

The author has granted a nonexclusive license allowing Library and Archives Canada to reproduce, publish, archive, preserve, conserve, communicate to the public by telecommunication or on the Internet, loan, distribute and sell theses worldwide, for commercial or noncommercial purposes, in microform, paper, electronic and/or any other formats.

The author retains copyright ownership and moral rights in this thesis. Neither the thesis nor substantial extracts from it may be printed or otherwise reproduced without the author's permission.
AVIS:

L'auteur a accordé une licence non exclusive permettant à la Bibliothèque et Archives Canada de reproduire, publier, archiver, sauvegarder, conserver, transmettre au public par télécommunication ou par l'Internet, prêter, distribuer et vendre des thèses partout dans le monde, à des fins commerciales ou autres, sur support microforme, papier, électronique et/ou autres formats.

L'auteur conserve la propriété du droit d'auteur et des droits moraux qui protège cette thèse. $\mathrm{Ni}$ la thèse ni des extraits substantiels de celle-ci ne doivent être imprimés ou autrement reproduits sans son autorisation.
In compliance with the Canadian

Privacy Act some supporting forms may have been removed from this thesis.

While these forms may be included in the document page count, their removal does not represent any loss of content from the thesis.
Conformément à la loi canadienne sur la protection de la vie privée, quelques formulaires secondaires ont été enlevés de cette thèse.

Bien que ces formulaires aient inclus dans la pagination, il n'y aura aucun contenu manquant.

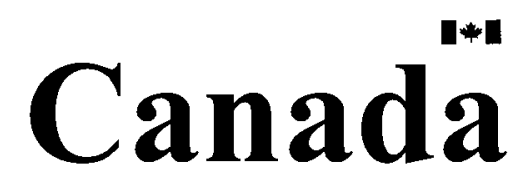




\section{Abstract}

Geometric errors in prostate cancer radiotherapy can affect patient outcome. Target delineation error, patient positioning error and internal target motion are three major sources contributing to the overall geometric uncertainty. In this thesis the quality of prostate target delineation, the global geometric error (vector sum of systematic error, organ motion, positioning error and collimator calibration error) and the intra-fraction organ motion during radiation delivery are studied. The effect of internal target motion and patient setup error on the tumour control probability and the normal tissue complication probability are calculated.

Anatomical images from the Visible Human Project were examined by an expert group who then segmented a gold standard volume for the subject prostate. On the corresponding CT images, six radiation oncologists were asked to each delineate the entire prostate organ twenty times, producing a total of 120 organ delineations. It was found that the physician delineated prostate volume is in general $30 \%$ larger than the "true" prostate volume, but this overestimate of volume only included $84 \%$ of the true prostate volume. It was also observed that there was a systematic error in the observer segmentations, such that none of the 120 segmentations ever included all of the posterior portion of the prostate, but all of the delineations extended past the anterior surface of the prostate.

Global geometric error and intra-fraction prostate organ motion were measured using data from 1028 portal images acquired over 257 fractions delivered to a total of 21 patients. In each patient, fudcial markers implanted into the prostate prior to treatment surrogated the position of the prostate. The overall geometric uncertainty was found to have a standard deviation of $5.3 \mathrm{~mm}$ for all observed directions. If both target delineation and target deformation uncertainties are included, the overall geometric uncertainty would be $6.3 \mathrm{~mm}$. The intra-fraction organ motion was found to have a standard deviation of $1 \mathrm{~mm}$. The effect of these geometric uncertainties on tumour control probability is simulated for the combinations of two treatment margins and three treatment techniques. 
To my wife Li Mei, my daughter Gao Xing and my son Gao Yi-shan for their loves 


\section{Acknowledgements}

I would like to thank my thesis advisor, Dr. Lee H. Gerig. His ideas, support and guidance kept me focused and always challenged me to see where is the limit and what else can be accomplished. Without his insightful suggestions and consistent encouragement, I would not have been able to complete this work. I am also grateful to Dr. Libni Eapen, Dr. Janos Szanto and Dr. David Wilkins for their valuable advices and useful discussions during the course of the project. I would like to thank my committee members Dr. Paul Johns and Dr. Gary Slater for all of their efforts in reviewing my thesis work.

Most importantly, I would like to thank my wife, Mei Li for everything she has sacrificed for me to make it this far in my life. For so many years of friendship and marriage, she has shown me extreme patience as well as offered unconditional love and support when I needed it most. I would also like to thank my daughter Xing and my son Yi-Shan for their love and understanding.

Last, certainly not the least, I would like to thank my parents and in-laws who supported me and my family throughout the years during my study. 


\section{Table of Contents}

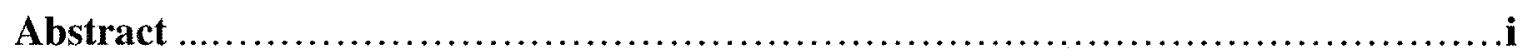

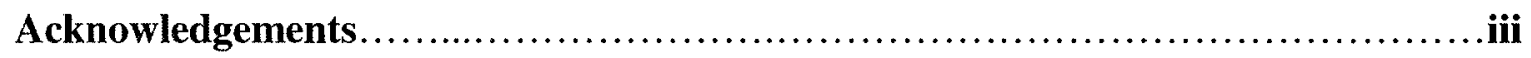

Table of Contents................................................................................................

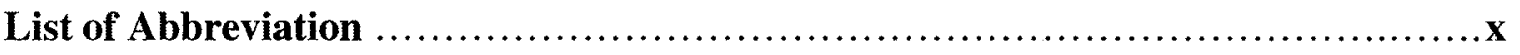

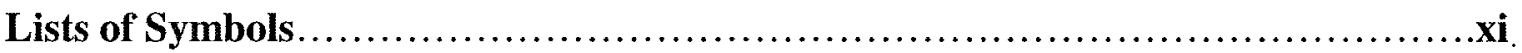

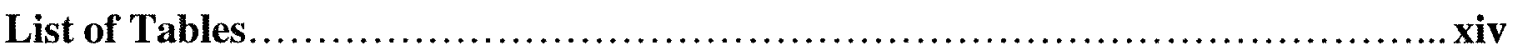

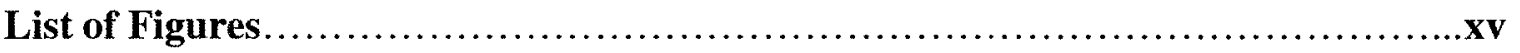




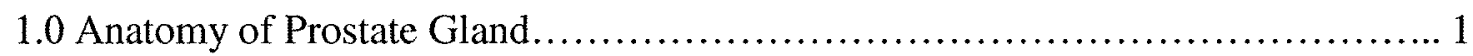

1.1 Uncertainties in Prostate Cancer Radiotherapy. .................................................. 2

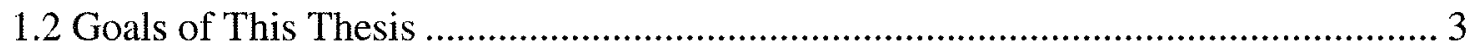

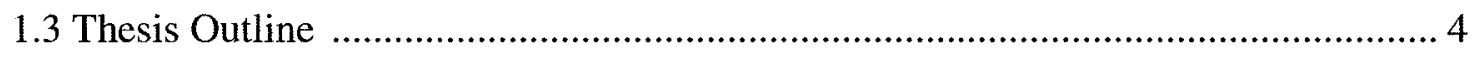

Chapter 2: Overview of Geometric Uncertainties in the Radiotherapy Process .....6

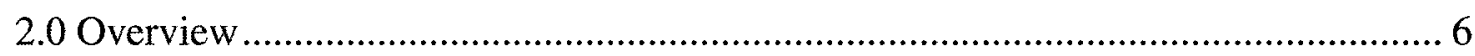

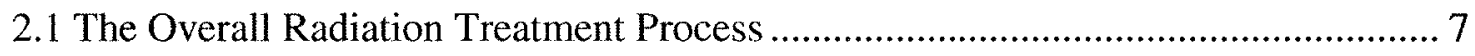

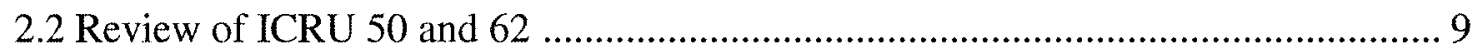

2.3 Review of Geometric Errors in Prostate Target Definition ............................... 13

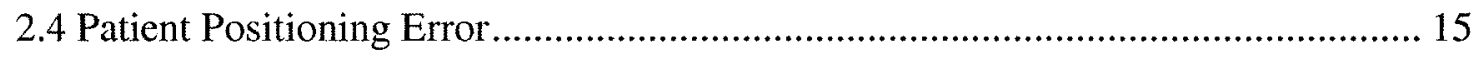

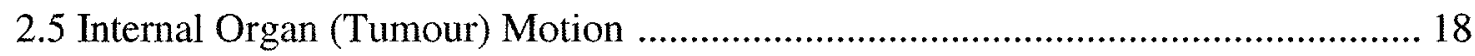

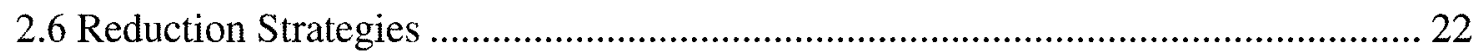

2.6.1 Conventional Target Motion Intervention ............................................. 22

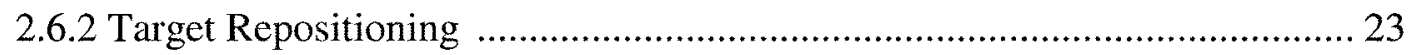

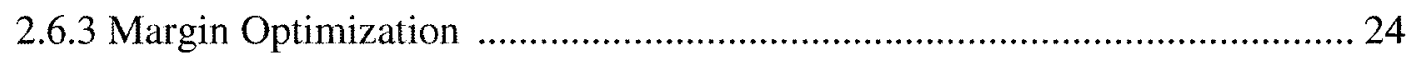

2.6.4 Intensity Modulated Radiation Therapy (IMRT) .................................. 27

2.6.5 Image Guided Radiation Therapy (IGRT) ........................................... 30

2.6.6 Helical Tomotherapy System ............................................................. 30

2.6.7 4D Conformal Radiotherapy and Radiation Gating ............................... 32

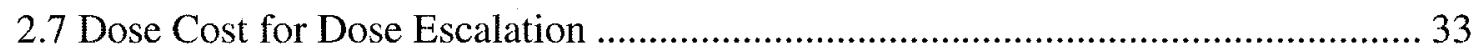

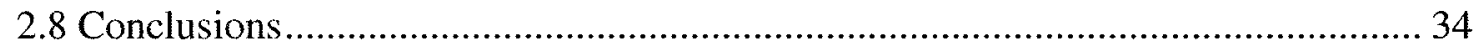

Chapter 3: Electronic Portal Images and Their Analysis Techniques 35

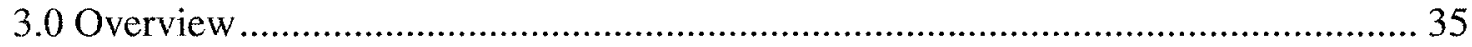

3.1 Introduction to the Linac-MLC_EPID Imaging System................................... 36

3.1.1 Linac Design and Geometry ............................................................. 36 


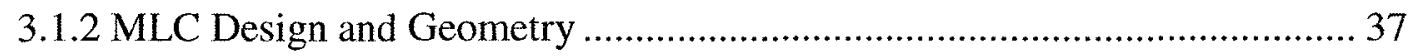

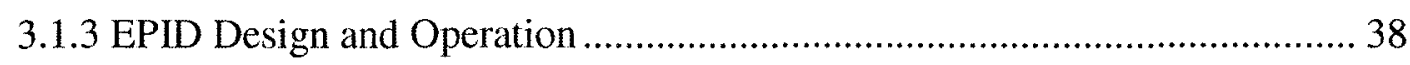

3.1.4 Information Regions in a EPID Image ............................................. 40

3.2 Image Enhancement and Process Tools Used in This Work .............................. 41

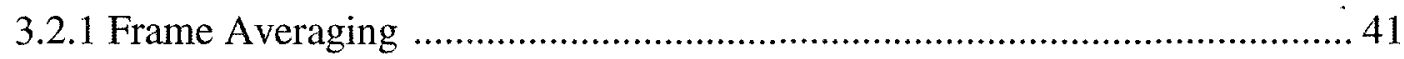

3.2.2 Contrast Limited Adaptive Histogram Equalization ............................... 42

3.2.3 Wavelet Based Contrast Enhancement................................................... 43

3.3 Algorithms for determining beam isocentre and field orientation........................ 47

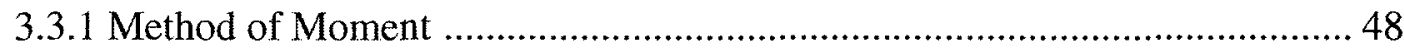

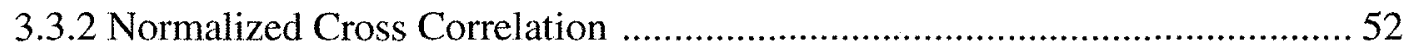

3.3.3 Line Pattern Detection Using the Radon Transform(Extraction of Interleaf

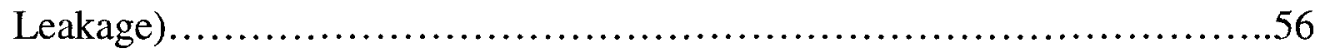

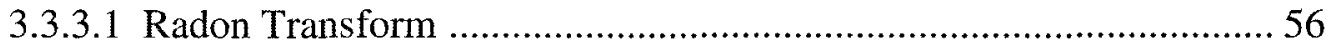

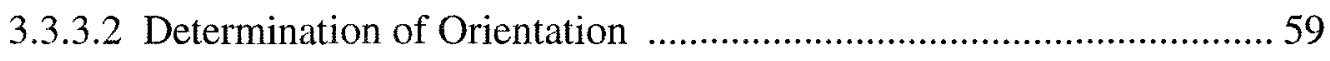

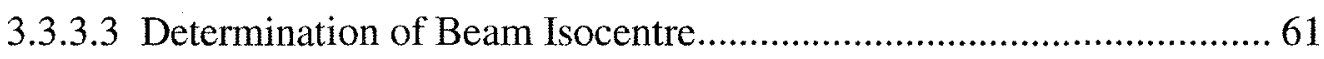

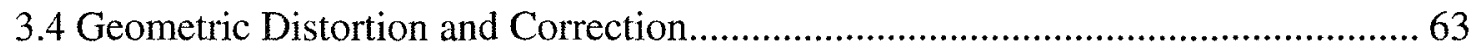

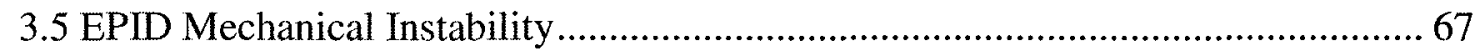

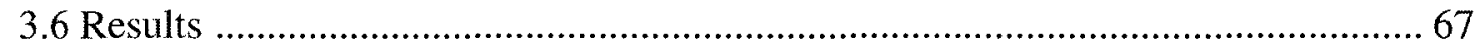

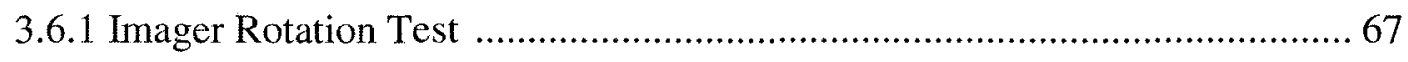

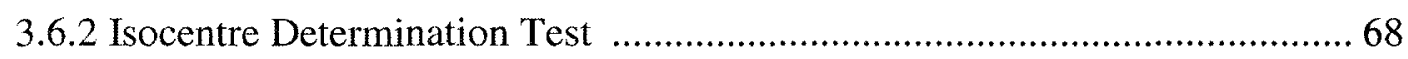

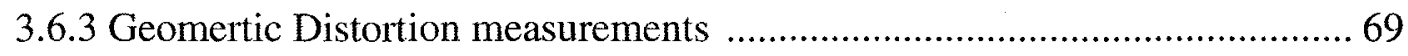

3.6.4 EPID Mechanical Stability Measurements.............................................. 71

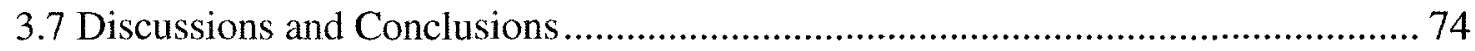

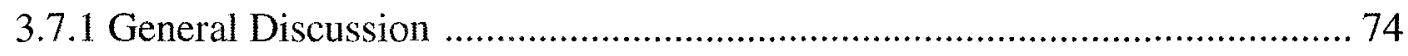

3.7.2 Effect of Distortion and Mechanical Stability on Beam Isocentre.............. 75

3.7.3 Effect of Distortion on Marker Positioning ............................................. 76

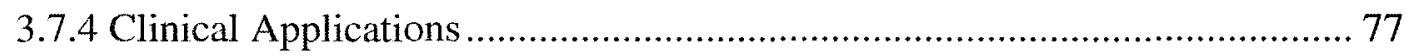

\section{Chapter 4: A Study of Prostate Delineation Referenced Against a Gold Standard} Created from the Visible Human ................................................. 78

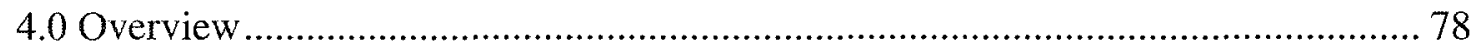




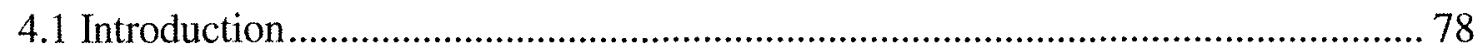

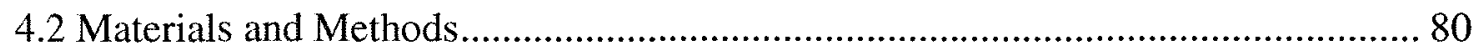

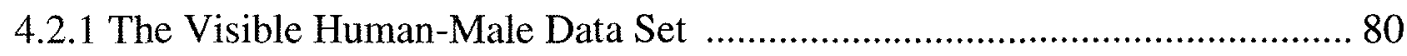

4.2.2 Observers and Gold Standard Group............................................................. 82

4.2.3 Determination of the Prostate Gold Standard Volume.................................... 82

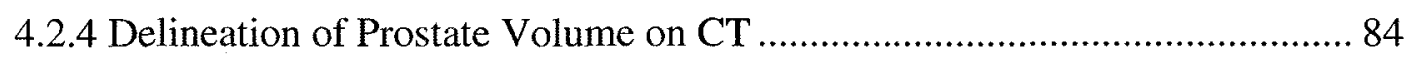

4.2.5 Image Registration .................................................................................. 84

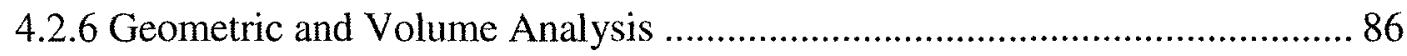

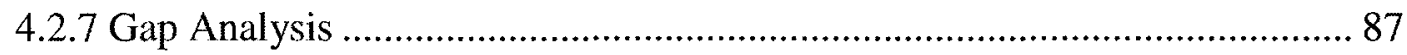

4.2.8 Quadratic Based Probability Volume Histograms ........................................ 88

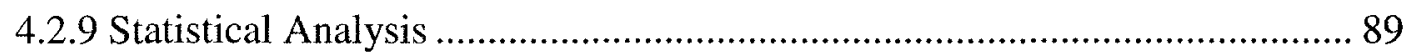

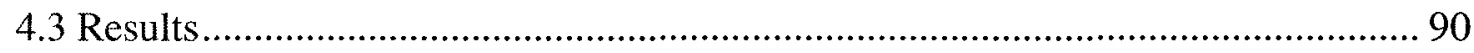

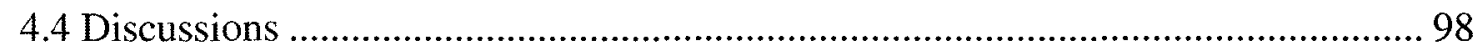

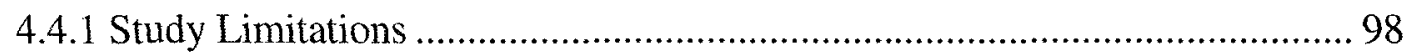

4.4.2 Comparisons with Other Studies ............................................................... 99

4.4.3 Margin Optimization ................................................................................ 100

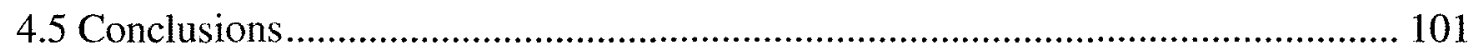

\section{Chapter 5: Quantifying Independent Geometric Uncertainties in Prostate Cancer}

Radiotherapy ............................................... 103

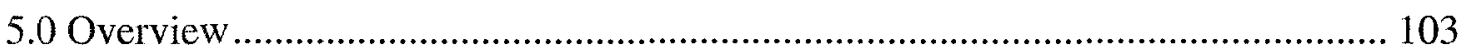

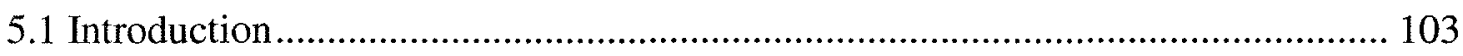

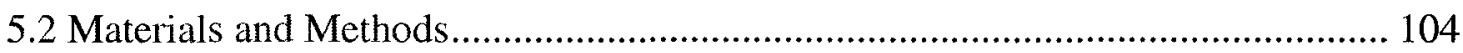

5.2.1 Study Population, Treatment Planning and Delivery ................................. 104

5.2.2 Image Enhancement and Correction for spatial distortion.......................... 105

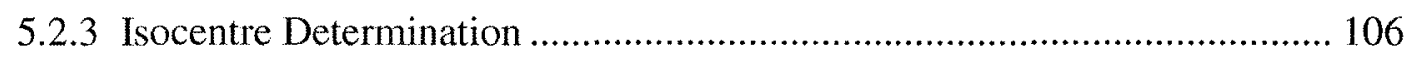

5.2.4 Extraction of Intra-fraction Organ Motion ................................................. 106

5.2.5 Determination of Global Geometric Error ................................................ 109

5.2.6 Determination of Patient Positioning Error ................................................ 110

5.2.7 Determination of Inter-fraction Organ Motion .......................................... 111

5.2.8 Determination of Collimator Calibration Error............................................ 111 
5.2.9 Statistics 112

5.3 Results and Analysis 113

5.3.1 An Example of Positioning and Global Geometric Error for a Specific Patient 116

5.3.2 Data Analysis for the Patient Population 116

5.3.2.1 Global Systematic Error and Random Uncertainty 116

5.3.2.2 Treatment Preparation and Treatment Delivery Uncertainties ....... 118

5.3.2.3 Intra-fraction Organ Motion 120

5.3.2.4 Inter-fraction Organ Motion and Inter-fraction Positioning Uncertainties 122

5.3.2.5 Examination of Supine and Prone Subpopulation........................ 122

5.3.3 Uncertainty in Collimator Calibration Error ........................................... 124

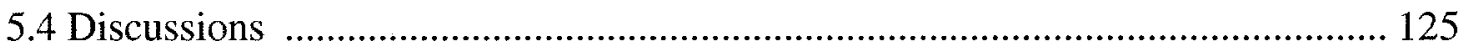

5.4.1 Oblique and Conventional Frame ........................................................ 125

5.4.2 Method Assumptions and Limitations ............................................. 126

5.4.3 Summary of Independent Geometric Uncertainties ............................... 127

5.4.4 Discussion of Systematic Error and Target Positioning Error .................. 128

5.4.5 Check or Seed Films ........................................................................ 129

5.4.6 Strategies to Reduce Individual Systematic Error................................... 131

5.4.7 Uncertainties in Preparation and Delivery Stages .................................. 132

5.4.8 Intra-fraction Organ Motion ............................................................... 133

5.4.9 Treatment Margin Optimization for Oblique Fields ................................ 134

5.4.10 Supine and Prone Treatment Position $\quad$..............137

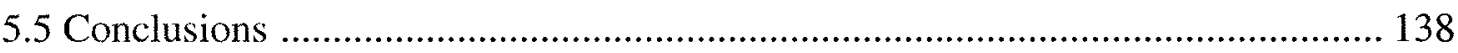

Chapter 6: Modeling the Biological Effect of Geometric Errors in EBRT ..........140

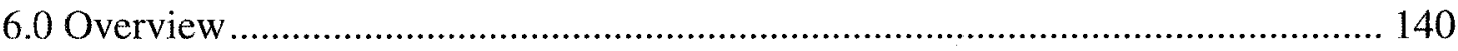

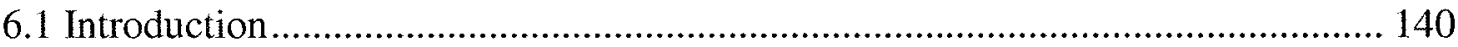

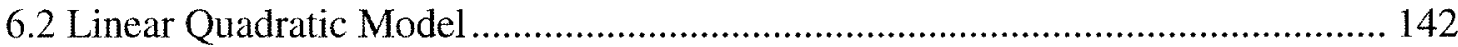

6.2.1 Linear Quadratic Model with Dose Protraction ...................................... 143

6.2.2 Fractionated Linear Quadratic Model ........................................... 143

viii 
6.2.3 Biological Effective Dose …………………………………………... 145

6.2.4 Equivalent Uniform Dose....................................................................... 146

6.2.5 Tumour Control Probability ………………………................................ 147

6.2.6 Normal Tissue Complication Probability ................................................... 148

6.3 Effect of Geometric Errors on Local Tumour Control ....................................... 152

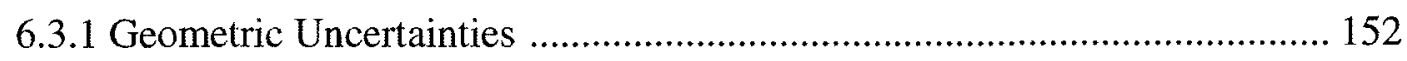

6.3.2 Radiobiological Parameters .................................................................. 153

6.3.3 Dosimetric Consequence of Geometric Error ……………………............ 155

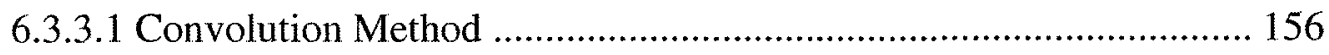

6.3.3.2 Monte Carlo Technique ………………………………………….... 157

6.3.4 Biological Effect of Geometric Error ...................................................... 158

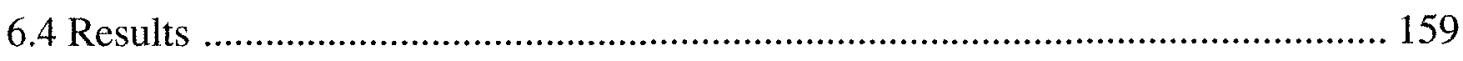

6.4.1 Patient and Treatment Planning ………………………………………... 159

6.4.2 Effect of Geometric Error ...................................................................... 163

6.4.3 Effect of Systematic Error .................................................................... 167

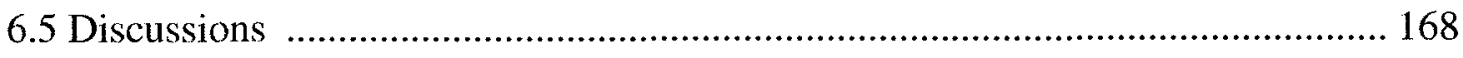

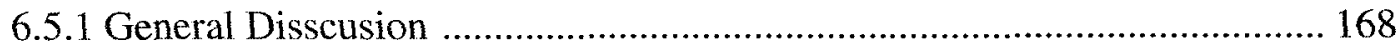

6.5.2 Alpha-Beta Ratio and Hypofractionation ……………............................ 168

6.5.3 Adaptive Radiotherapy ...................................................................... 171

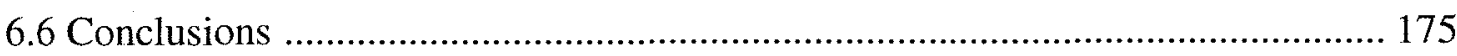

Chapter 7: General Conclusions and Scope for Future Work ....................176

7.1 General Conclusions ..................................................................................... 176

7.1.1 Conclusions from Geometric Uncertainty Measurements …....................... 176

7.1.2 Conclusions from Prostate Delineation Study............................................. 177

7.1.3 Conclusions from modeling of geometric uncertainties for prostate cancer 179

7.2 Scope for Future Work............................................................................ 180

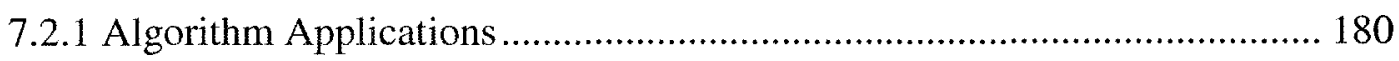

7.2.2 Target Positioning ................................................................................... 181

7.2.3 Future Research on Target Delineation ................................................. 181

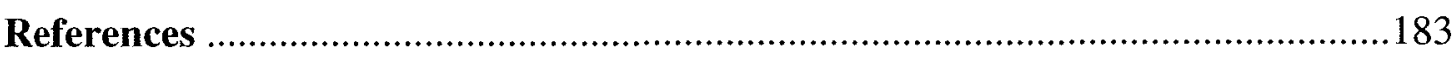

ix 


\section{Lists of Abbreviations}

$\begin{array}{ll}\text { 3DCRT } & \text { 3 Dimensional Conformal Radiation Therapy } \\ \text { 4DRT } & \text { 4 Dimensional Radiation Therapy } \\ \text { AP } & \text { Anterior-Posterior direction } \\ \text { BED } & \text { Biological Effective Dose } \\ \text { CLAHE } & \text { Contrast Limited Adaptive Histogram Equalization } \\ \text { COM } & \text { Centre of Mass } \\ \text { CT } & \text { Computed Tomography } \\ \text { CTV } & \text { Clinical Target Volume } \\ \text { DNA } & \text { Deoxyribose Nucleic Acid } \\ \text { DVH } & \text { Dose Volume Histogram } \\ \text { EBRT } & \text { External Beam Radiation Therapy } \\ \text { EPID } & \text { Electronic Portal Image Device } \\ \text { EUD } & \text { Equivalent Uniform Dose } \\ \text { FSU } & \text { Functional Sub-Unit } \\ \text { GC } & \text { Geometric Center } \\ \text { GTV } & \text { Gross Tumour Volume } \\ \text { ICRU } & \text { International Commission on Radiation Units } \\ \text { IM } & \text { Internal Margin } \\ \text { IMRT } & \text { Intensity Modulated Radiation Therapy } \\ \text { IGRT } & \text { Image Guided Radiation Therapy } \\ \text { LQ } & \text { Linear Quadratic } \\ \text { LR } & \text { Left-Right direction } \\ \text { LO } & \text { Left Oblique direction } \\ \text { MC } & \text { Monte Carlo } \\ \text { MHF } & \text { Mexican Hat Function } \\ \text { MLC } & \text { MultiLeaf Collimator } \\ \text { MRI } & \text { Magnetic Resonance Imaging } \\ \text { NTCP } & \text { Normal Tissue Complication Probability } \\ \text { OAR } & \text { Organ At Risk } \\ \text { PET } & \text { Positron Emission Tomography } \\ \text { PDF } & \text { Probability Density Function } \\ \text { PTV } & \text { Planning Target Volume } \\ \text { SD } & \text { Standard Deviation } \\ \text { SI } & \text { Superior-Inferior direction } \\ \text { SM } & \text { Setup Margin } \\ \text { SNR } & \text { Signal-Noise ratio } \\ \text { TCP } & \text { Tumour Control Probability } \\ \text { TPS } & \text { Treatment Planning System } \\ \text { TOHRCC The Ottawa Hospital Regional Cancer Center } \\ \text { UTCP } & \text { Uncomplicated Tumour Control Probability } \\ \text { VHP } & \text { Visible Human Project } \\ & \end{array}$




\section{List of Symbols}

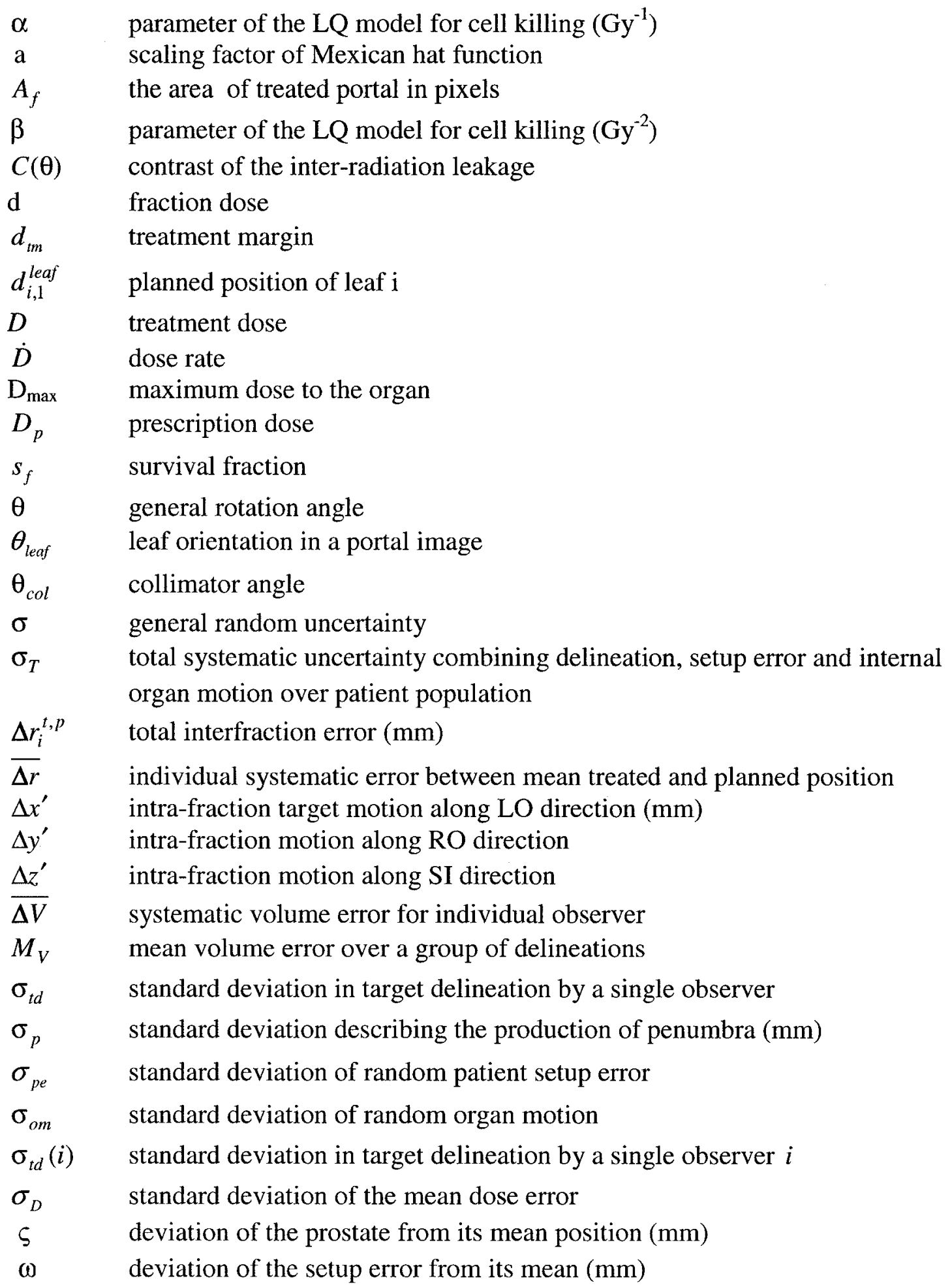




\begin{tabular}{ll}
$\xi_{i}^{t}$ & seed deviation from its mean position (mm) \\
$\lambda$, & a parameter describing effective half-time for cell repair $\tau=0.693 / \lambda$. \\
$\Sigma$ & general systematic uncertainty for a group \\
$\Sigma_{T}$ & total systematic uncertainty combining delineation, setup error and motion \\
$\Sigma_{t d}$ & Inter-observer uncertainty in volume delineation \\
$\rho_{c}(x, y, z)$ & tumour cell density at coordinate $(\mathrm{x}, \mathrm{y}, \mathrm{z})$ \\
$\Psi$ & Mexican hat function in frequency space \\
$\Psi$ & Mexican hat function in position space \\
$f_{T I}$ & percentage of "true" target included in an individual delineation \\
$f(x, y)$ & intensity map of a portal image at location $(i, j)$ \\
$g_{\theta}(s)$ & Random transform of an image along $\theta$ direction \\
$g(i, j, k)$ & number of times that a voxel $(i, j, k)$ is being included in physician-delineated \\
$M_{c t}$ & volume \\
$M_{f}$ & Ratio of the spatial resolution between two CT and photographic modalities \\
$m_{n t}$ & magnification factor for a portal image \\
$m_{x, y}, n_{y}$ & the slope parameter of the dose-response curve for normal tissue \\
$M_{V}$ & overall volume difference between the mean delineated and gold standard \\
$N_{0}$ & number of initial tumour cells \\
$n_{f}$ & number of fractions \\
$\mathrm{K}$ & map of cross correlation between two functions \\
$\Theta$ & mean geometric distortion \\
$\mathrm{x}$ & position along LR axis (mm) \\
$x^{\prime}$ & position along LO axis (mm) \\
$x_{c t}$ & position along LR axis in CT frame \\
$x_{p h}$ & position along LR axis in anatomical photography frame \\
$x_{i, 1}^{l e a f}$ & position of leaf i in the film coordinate system \\
$\mathrm{y}$ & position along AP axis (mm) \\
$y^{\prime}$ & position along RO axis (mm) \\
$y_{c t}$ & position along AP axis in CT frame \\
$y_{p h}$ & position along AP axis in anatomical photography frame \\
$\mathrm{z}$ & position along SI axis (mm) \\
$z^{\prime}$ & position along SI axis (mm) \\
$\mathrm{V}$ & general volume (cm ${ }^{3}$ ) \\
$V_{G S}^{C T}$ & common agreed volume between a CT delineated target and the "true" gold \\
$V_{G S}$ & standard prostate volumes \\
$v_{i}$ & volume defined by the expert group \\
& subvolume of tumour \\
\hline
\end{tabular}


$v_{\text {eff }} \quad$ effective volume

$w \quad$ width of a leaf in $\mathrm{mm}$

$\mathrm{TD}_{50}$ dose delivered to entire volume of the normal tissue leading to $50 \%$ kill rate 


\section{List of Tables}

Table 2.1: Summary of delineation uncertaqinty studies:. ........................................ 15

Table 2.2: Summary of patient setup errors in pelvic sites....................................... 17

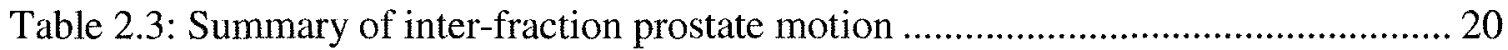

Table 2.4: The radiation dose required for image guided radiotherapy .......................... 34

Table 3.1: Comparison of different measures for inter-leaf leakage analysis ................. 68

Table 3.2: Deviations from true isocentre for three methods ......................................69

Table 3.3: Geometric distortion means and ranges $(\mathrm{mm})$ for different gantry angles...... 70

Table 3.4: Distribution of the beam isocentre for different gantry angles ...................... 74

Table 4.1: Mean prostate volume, encompassing volume and common agreed volumes 91

Table 4.2: Geometric displacements along LR,AP and SI directions............................93

Table 4.3: Gaps ( $\mathrm{mm}$ ) between delineations and gold standard in four quadrants.......... 95

Table 5.1: Summary of global geometric errors in three observed directions................ 117

Table 5.2: Treatment preparation and treatment delivery uncertainties ........................ 118

Table 5.3: Intra-fraction organ motion: results of 257 fractions............................. 120

Table 5.4: Uncertainties in inter-fraction organ motion and patient positioning

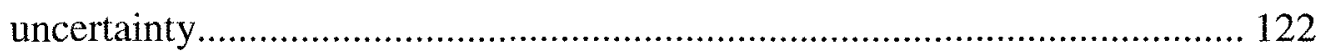

Table 5.5: MLC/Collimator calibration uncertainties .............................................. 125

Table 5.6: Summary of the individual uncertainties ............................................ 127

Table 6.1: Parameter values for NTCP calculation ............................................... 150

Table 6.2: Summary of geometric uncertainties in overall treatment process .............. 152

Table 6.3: Parameter sets used in the calculations of tumour control probability for external beam radiotherapy ...............................................................153

Table 6.4: Summary of NTCP for different fractionated schemes...................171

Table 6.5: Summary of TCP for different fractioned schemes ......................171 


\section{List of Figures}

Figure 1.1: The anatomy of prostate gland ...............................................................

Figure 2.1: Flowchart of the overall radotherapy process for prostate cancer................. 6

Figure 2.2: Graphic representations of the GTV, CTV, PTV and OAR and their

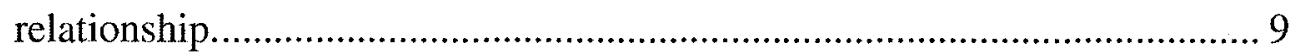

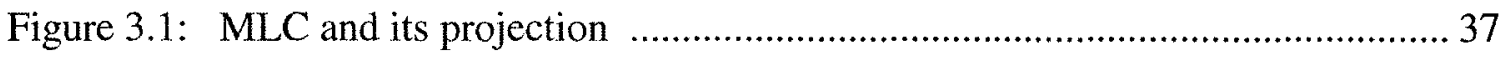

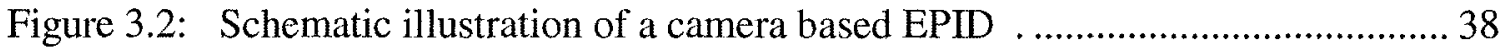

Figure 3.3: Linear attenuation coefficients as a function of photon energy for

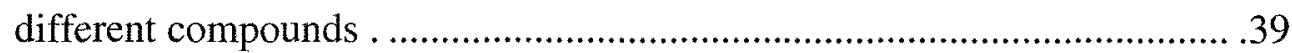

Figure 3.4: Diagram of Information segmentations in an EPID portal image ............. 41

Figure 3.5: Surface and contour plots of the Mexican Hat function in position space .. 43

Figure 3.6: Surface and contour plots of the Mexcian hat function in frequency space 44

Figure 3.7: Enhancement of fiducial markers using three techniques................. 46

Figure 3.8: Histogram of a portal image (a) and its cumulative distribution (b) ............ 51

Figure 3.9: (a) original portal image (b) binary format after thresholding ..................52

Figure 3.10: Normalized cross correlation between a templete and a portal image........ 56

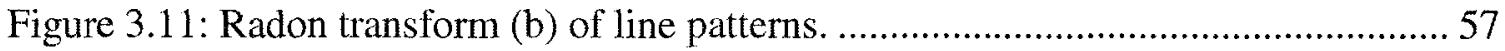

Figure 3.12: (a) Portal image contianing inter-leaf leakage and (b) the corresponding sinograph plot from the Radon transform............................................. 58

Figure 3.13: Radon transform of inter-leaf leakage at $0^{\circ}$ and $90^{\circ}$...............................59

Figure 3.14: Contrast of inter-leaf leakage as a function of projection angle................... 61

Figure 3.15: The Superposition of 18 images of leaf $15^{\text {th }}$ at 18 collimator angles 64

Figure 3.16: Composite image of an MLC configuration taken at collimator $0^{\circ}$ and $90^{\circ}$

Figure 3.17: Superposition of two opposed portal images without (a) and with (b) correction for spatial distortion 
Figure 3.18: The isocentre displacement as a function of gantry angle 72

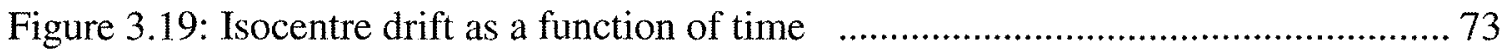

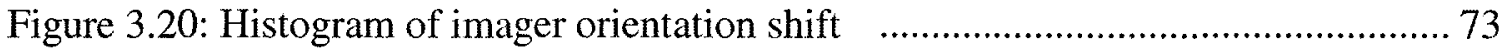

Figure 4.1: (a)An axial photo image of plevic region and (b)corresponding CT ......... 81

Figure 4.2: Rendering of the gold standard prostate and rectum ................................ 83

Figure 4.3: Rendering of the gold standard prostate after smoothing.......................... 83

Figure 4.4: Checkerboard of the CT and photo images following $2 \mathrm{D}$ fusion................ 85

Figure 4.5: Definitions of the encompassing and common agreed volumes ................. 86

Figure 4.6: Gap representation between gold standard and corresponding delineated

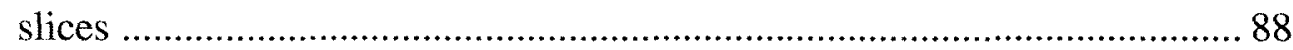

Figure 4.7: Histogram of the delineated prostate volumes ........................................ 92

Figure 4.8: Histogram of the percentage of true volume included in each prostate

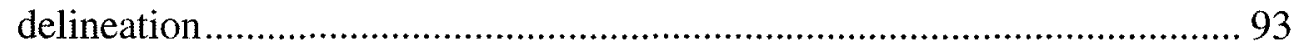

Figure 4.9: Centroid displacements in three orthognal directions ............................. 94

Figure 4.10: The mean and standard deviation of the gap as a function of prostate slices from base to apex for four quadrants ................................................ 95

Figure 4.11: Volume definition histogram for prostate volume for four quadrants ....... 96

Figure 4.12: Volume definition histogram for non-target volume in four quadrants ...... 97

Figure 4.13: Probability volume histograms for different margins ........................... 97

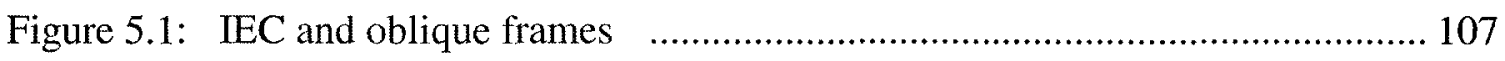

Figure 5.2: Opposed image pairs used to determine intra-fraction prostate motion $\quad . .108$

Figure 5.3: Global geometric error and positioning error in orthoganal directions..... 114

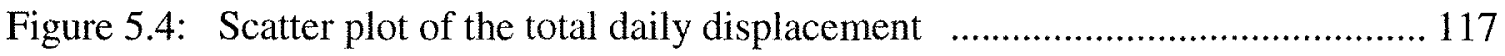

Figure 5.5: Individual systematic error in three observered directions ..................... 118

Figure 5.6: Intra-fraction organ motions in three observed fractions ...................... 120

Figure 5.7: Scatter plot of two types of errors for supine (a) and prone (b) patients 123

Figure 5.8: Systematic error verus random error ............................................. 128

Figure 5.9: Percent of systematic error removed as a function of number of simulations

Figure 6.1: Surviving fraction as a function of dose for normal and tumour tissues ... 145

Figure 6.2: Relationship among TCP, NTCP and UTCP .................................... 151 
Figure 6.3: TCP as a function of dose.

Figure 6.4: Flowchart of the treatment simulation in the presence of geometric error 159

Figure 6.5: Planned dose distribution in the pelvic region, The white represents the prostate CTV while gray represents the rectal wall ............................. 160

Figure 6.6: Dose-volume histograms for the rectal wall......................................... 161

Figure 6.7: Dose-volume histograms for prostate CTV ........................................ 162

Figure 6.8: TCP and NTCP as a function of prescription dose for two typical margins (No clinical intervention applied) ..................................................... 164

Figure 6.9: TCP and NTCP as a function of prescription dose for two typical margins (Positioning error corrected)

Figure 6.10: TCP and NTCP as a function of prescription dose for two typical margins

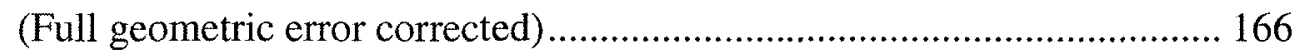

Figure 6.11: TCP as a function of systematic uncertainty ...................................... 167

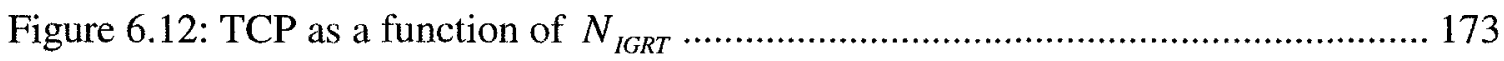

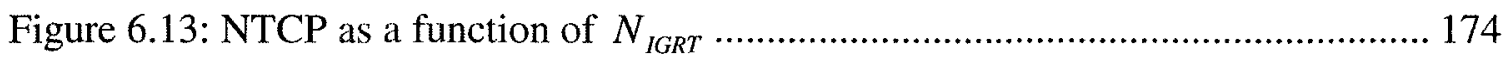




\section{CHAPTER 1 \\ General Introduction}

\subsection{Anatomy of Prostate Gland}

The prostate gland, illustrated in Figure 1.1, lies deep in the pelvis. It surrounds the urethra where the urine tube runs from the bladder, through the prostate. In healthy men it is usually the size of a walnut, but may grow larger with age. The weight of the normal prostate gland is about 15 20 grams and it is partly muscular and partly glandular, with ducts opening into the prostatic portion of the urethra. The primary function of prostate gland is to secrete a slightly alkaline fluid that forms the seminal fluid that carries sperm. Prostate cancer occurs when cells of the prostate mutate and begin to multiply out of control. Prostate cancer may cause pain, difficulty in urinating, erectile dysfunction and other symptoms.

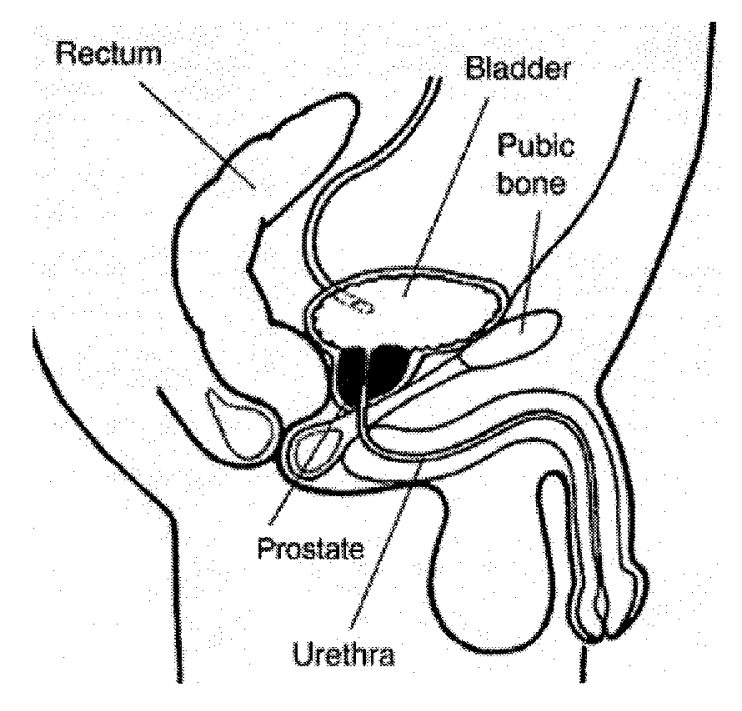

Figure 1.1 The anatomy of prostate gland ${ }^{[1]}$ 


\subsection{Uncertainties in Prostate Cancer Radiotherapy}

Prostate cancer is the most commonly diagnosed male cancer in Canada and similarly in United States. Cancer Statistics Canada ${ }^{[2]}$ reported that $26.8 \%$ of all male cancers were prostate cancer in 2003. Prostate was the third most lethal cancer after lung and colorectal cancer in Canadian males. Currently there are three major treatment methods for prostate cancer: radiotherapy, surgery and chemotherapy. Radiotherapy is an effective approach to treating cancer using high-energy ionizing radiation. In this method, ionizing radiation such as high-energy photons produced by medical linear accelerators, deposit energy that destroys cancerous cells within a tissue volume. The lethal damage to a cell is on the genetic material, making it impossible to reproduce (biological cell death). However, this damage occurs to both cancerous cells and healthy cells. In cancer research, Tumour Control Probability (TCP) and Normal Tissue Complication Probability (NTCP) are used to quantify the damage to tumour and normal tissues, respectively.

It is commonly accepted that the individual sub-processes that constitute external beam radiation therapy can affect patient outcome, and specifically geometric uncertainties play a major role in tumor control and normal tissue complications. These uncertainties span the entire treatment process include tumour definition, inter- and intra-fraction patient motion, organ motion, patient positioning variation and linac geometric uncertainties, etc. Many strategies have been developed to reduce these uncertainties and to ensure full target coverage over the course of treatment. Some strategies emphasize improved target definition by $\mathrm{CT}$, MR and recently functional imaging. ${ }^{[3-5]}$ Others suggest the improvement in dosimetric accuracies and treatment planning algorithms ${ }^{[6-8]}$ and advances in dosimetric protocols such as TG51. ${ }^{[9]}$ Linac based uncertainties are addressed through improved linac design and control systems and through enhanced clinical quality assurance programs. ${ }^{[10]}$ Positioning uncertainties have been addressed through a variety of treatment techniques including fiducial markers, ${ }^{[11-13]}$ patient immobilization, ${ }^{[14,15]}$ and electronic portal imaging ${ }^{[16-18]}$ and image guided techniques. In spite of these advances geometric uncertainties are still mitigated by adding a treatment margin ${ }^{[19,20]}$ to ensure a full dose coverage to the tumour target. 
For low risk prostate cancer where tumour cells are confined to the prostate itself, target delineation, patient positioning variation, and internal target motion are three major sources contributing to the global geometric uncertainty. However, the knowledge of these uncertainties and their effect on tumour control are not well known. Investigating these issues is the aim of this work.

In this thesis, "error" and "uncertainty" are generally used to describe the geometric factors in the radiotherapy process. The error is defined as the difference between a measured value for one variable and its true value or its group mean, while the uncertainty describes the standard deviation of the variable. Please note the uncertainty sometimes may be interchanged with error to describe the source of the examined error or uncertainty.

\subsection{Goals of This Thesis}

The goals of this thesis are:

- To investigate intra- and inter- observer variability in prostate organ delineation, and importantly to measure systematic difference between CT delineated prostate volume and the true prostate volume using the data from the Visible Human Project (VHP); ${ }^{[21]}$

- To measure and quantify day-to-day prostate organ motion using portal imaging of implanted fiducial markers;

- To model the effect of individual geometric errors on local tumour control probability and normal tissue complication probability;

- To compare and recommend strategies to reduce the geometric uncertainty in prostate radiotherapy. 


\subsection{Thesis outline}

This thesis describes our research work, performed at The Ottawa Hospital Regional Cancer Centre (TOHRCC), on geometric uncertainty in prostate cancer radiotherapy.

Chapter two is an overview of the entire radiation therapy treatment process for prostate cancer and associated geometric uncertainties. Target delineation, patient positioning error and internal target motion are emphasized. The cause, nature and magnitude of these errors are reviewed separately. This chapter also summarizes current treatment techniques for prostate cancer.

Much of this work uses medical images to measure and quantify the geometric error. These images include electronic portal images acquired during radiation delivery and CT images acquired during treatment simulation. However, the contrast of the CT and portal images is very low and required significant image enhancement and correction before the useful information could be extracted. Chapter three discusses electronic portal imaging and the image processing techniques used in this work. Part of the Chapter three was sent to the Journal of Applied Clinical Medical Physics and is currently under peer reviewed.

Chapter four addresses systematic and random errors in target delineation. The uncertainty in target delineation is a significant source of geometric uncertainty in prostate cancer radiotherapy, but is poorly determined or defined due to the lack of a well-accepted "gold standard". In this work, we take advantage of data from the Visible Human Project (VHP) which provides a set of co-registered CT and axial anatomical photographic images of the human male. An expert group consisting of an urologist, a radiation oncologist and an anatomist defined the true or gold standard volume of the prostate on the anatomical images of a male cadaver. The corresponding CT images were provided to six radiation oncologists who each contoured the entire prostate 20 times. Each of the 120 definitions was subsequently compared against the gold standard. The intra- and inter-observer uncertainties were then measured and are described in chapter 
four. The material of the chapter is under preparation for a manuscript for British Journal of Radiology.

Chapter five describes the measurement and calculation of treatment geometric errors of the prostate from its expected position during 257 fractions of radiation delivery to 21 patients implanted with fiducial seeds. During dose delivery, portal images of each of the 4 oblique treatment fields were acquired with a Siemens Beamview TI (Concord, CA) electronic portal imager. Assuming that the positions of the fiducial seeds are fixed within the prostate, the coordinates of three seeds are back projected to the treatment room coordinate system and then transformed to the CT coordinate system for comparison. Following seed localization, determination of the beam isocentre as well as the shift in reference bony landmarks, three-dimensional inter- and intra-fraction organ motion can be determined. The material of Chapter five is under preparation for a manuscript which will submit to International Journal in radiation oncology, biology and physics.

The aim of radiotherapy is to maximize tumour cell kill while minimizing normal tissue damage. Geometric error compromises our ability to achieve this. Chapter six describes the effect of the target motion and patient positioning error on the tumour control probability and normal tissue complication probability.

Chapter seven is a general summary of this thesis and the future work needed to be done for the improvement of prostate cancer treatment. 


\section{CHAPTER 2 \\ Overview of Geometric Uncertainties in the Radiotherapy \\ Process}

\subsection{Overview}

Geometric uncertainties are major factors in tumour control and normal tissue complication for prostate cancer. Understanding these uncertainties is a prerequisite for the improvement of patient outcome. This chapter discusses the various geometric uncertainties in the radiotherapy process and several strategies used to reduce their impact on local tumour control. Section 2.1 describes the overall radiation treatment process for prostate cancer. Section 2.2 summarizes ICRU report 50 and its supplementary report 62 . The concepts of GTV, CTV and PTV and their relationships are presented. Target definition uncertainty, day-to-day patient positioning uncertainty and internal organ (or target) motion are independently reviewed in sections $2.3,2.4,2.5$, respectively. The causes, nature and magnitudes of these uncertainties are also summarized. Section 2.6 reviews current treatment techniques including 3DCRT, IMRT, IGRT and 4DRT. Section 2.7 summarizes the dose resulting from different image-guided strategies. 


\subsection{The Overall Radiation Treatment Process}

In general, there are multiple steps (simulation, planning, delivery and verification) ${ }^{[22]}$ involved in the treatment of prostate cancer. The flowchart of this process is illustrated in Figure 2.1.

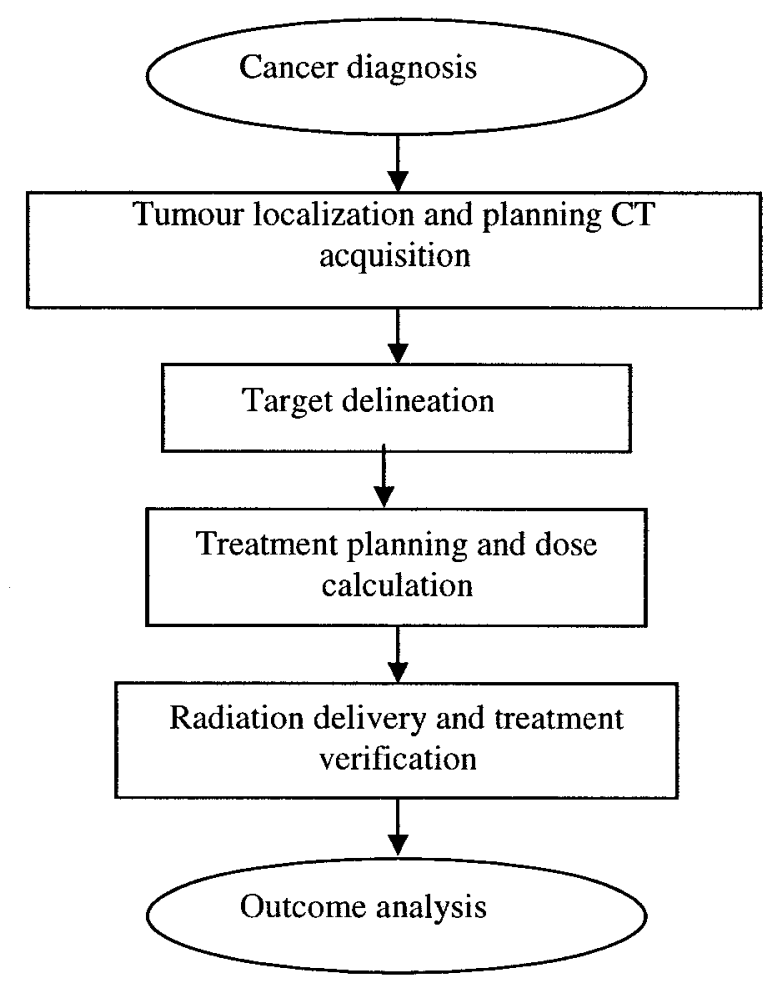

Figure 2.1 Flowchart of the overall radiotherapy process for prostate cancer

When a patient is diagnosed with prostate cancer, he may have an initial consultation with a radiation oncologist. Based on diagnosis, staging and general health, the radiation oncologist will determine whether radiation therapy is an effective treatment option. The treatment process is initiated when the radiation oncologist and the patient agree to proceed with radiation therapy. A radiotherapy process starts with the treatment planning stage. The first step of treatment planning is to acquire a three-dimensional image of the patient's anatomy. Typically this is done using a CT (Computed Tomography) scan. 
During the image acquisition, the patient is immobilized in the treatment position and skin markers are applied.

The CT images are then used to generate an outline of the patient's external body shape. The radiation oncologist may also delineate the target volume to be treated and choose an isocentre within the defined target. Often other modalities such as Magnetic Resonance Imaging (MRI) or Positron Emission Tomography (PET) are used to help define the extent of disease and to determine the target volume. Following treatment simulation, the CT image set and associated target contours are imported into a treatment planning system for the generation of a treatment plan. Uncertainty in the target delineation should be considered when a treatment plan is created. The geometric uncertainty in the prostate target delineation is reviewed in section 2.3.

Once the total dose and number of fractions have been prescribed by the radiation oncologist, the treatment plan is optimized for beam type, energy, field size, gantry angle and entry ports using sophisticated planning software. When the patient is ready to be treated with external beam radiotherapy, patient data (e.g. couch position, skin marker aligned to the beam) is transferred from the planning computer to the treatment machine (e.g. linear accelerator). A typical treatment course for prostate cancer consists of 20 40 daily fractions over a period of $4 \sim 8$ weeks. During treatment the patient is repositioned on the treatment couch many times. Day-to-day patient positioning errors will occur and these errors modify the dose distribution to the target volume. Variation in the patient positioning is reviewed in section 2.4. For targets such as the prostate, the target position within the patient relative to the bony anatomy may change during the course of radiation delivery, from one fraction to another or from field to field. These changes represent inter- and intra-fraction target motion, respectively. The effect of inter \& intra-fraction target motion is discussed in section 2.5.

Immediately prior to or after radiation delivery, the treatment accuracy is frequently verified through radiographic portal imaging. Patient outcome following radiation treatment can be assessed by either Dose Volume Histograms (DVH) or Tumour Control 
Probability (TCP). The effect of the treatment errors on local tumour control is investigated in chapter 6 .

\subsection{Review of ICRU Reports 50 and $62^{[19,20]}$}

The goal of external beam radiotherapy is to maximize the radiation dose to the tumour, while sparing the surrounding healthy tissues, particularly Organs At Risk (OAR). In order to achieve this, the International Commission on Radiation Units (ICRU) had released two reports (ICRU report 50 and supplementary report 62) offering recommendations and strategies to incorporate treatment uncertainties, particularly for 3 Dimensional Conformal Radiotherapy (3DCRT). In the two reports, the ICRU recommends a dosimetric tolerance within $+7 \%$ and $-5 \%$ to a well-defined prescription point within the tumour target. However, the tolerance for geometric uncertainty was not specified.

In ICRU report 50, several concepts for tumour volume are defined:

The Gross Tumour Volume (GTV) is the volume defined by the gross palpable or demonstrable tumour extent. The shape, size and location of the GTV may be determined by different diagnostic methods, e.g., CT, Magnetic Resonance Imaging (MRI), or Positron Emission Tomography (PET) as well as physical examination. Since the tumor cell density in the GTV is usually higher than elsewhere, it often requires a large radiation dose to destroy all tumour cells within its volume.

The Clinical Target Volume (CTV) is a tissue volume containing a demonstrable GTV and/or sub-clinical microscopic malignant disease. The determination of a CTV requires considerations such as the local invasive capacity of the tumor and its potential to spread during the period from the diagnosis to the treatment. This volume must be treated adequately in order to achieve the cure. In some cases a dose gradient over the volume may be desired and this can be achieved by various 3DCRT and IMRT techniques. 
The definitions of the CTV and GTV are not limited to external beam radiotherapy, and are based on general oncology principles and the experience and knowledge of radiation oncologists. Once the CTV is determined, the radiation oncologist will prescribe a dose contour or dose distribution over the CTV volume to achieve a certain treatment goal (e.g. cure, palliation, local tumor control). When prescribing the dose to the CTV, radiation sensitivities of surrounding healthy tissues and OARs are often considered. Due to day-to-day patient positioning error and internal organ motion, it is almost impossible to deliver the prescribed dose to CTV if only the CTV is defined as the treating volume. This limitation leads to the concept of Planning Target Volume (PTV).

The Planning Target Volume (PTV) is the volume chosen to ensure that the prescribed dose is actually delivered to the CTV. In defining this volume, factors such as appropriate beam size, beam arrangement, and some geometric uncertainties in the radiotherapy process should be considered. However, only uncertainties in set up error,

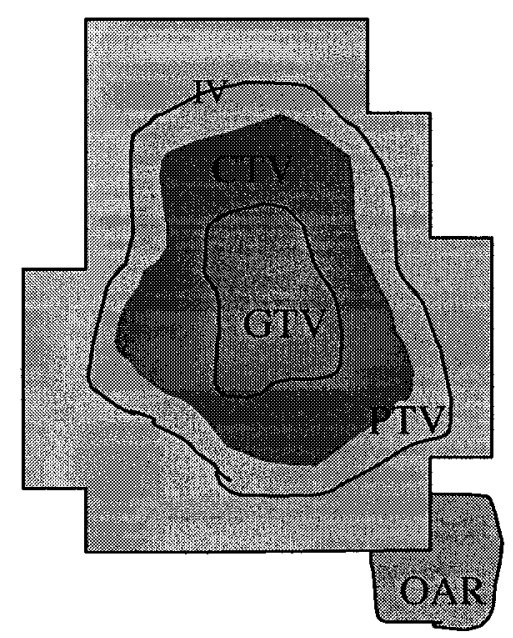

Figure 2.2 Graphic representations of the GTV, CTV, PTV and OAR, and their relationship.

internal organ motion, and machine performance related uncertainties are addressed in the ICRU reports. The expansion of the CTV generally depends on direction (e.g. Ant/Post, Sup/Inf), patient characteristics (age, weight and general health), organ or disease site (e.g. close to lung) and technology used (e.g. immobilization technique). The 
radiation oncologist usually balances treating volume and normal tissue toxicities in a comprehensive way. The relationship among GTV, CTV, PTV and adjacent OAR is shown in Figure 2.2.

As a supplement to ICRU report 50, ICRU report 62 provides guidance to optimize the planning target volume for external beam radiotherapy. In this report, internal organ motion and daily patient setup error are considered as two major uncertainties that may compromise dose delivery to the CTV. Three scenarios (A, B, and C) for PTV are suggested.

In scenario A, to ensure that all parts of the CTV receive the prescribed dose during the treatment, additional safety margins for geometric variations and errors must be taken into account. An Internal Margin (IM) is added for the possible target variations and the deformation in shape and size of the CTV relative to the bony anatomy. In addition, a Set-up Margin (SM) is further added to cover all potential variations/uncertainties in patient position relative to the radiation beam. Based on scenario $A$, the PTV can be represented as:

$$
P T V=C T V+I M+S M
$$

Note that the PTV, CTV, IM, and SM presented in Eq 2.1 are all volume concepts. The simple linear addition of all geometric uncertainties could lead to an excessively large PTV that would encompass large amounts of surrounding normal tissue volumes or OARs, potentially causing significant normal tissue complication.

In scenario $\mathrm{B}$, the treatment margin is calculated as the quadratic sum of two geometric uncertainties. With an assumption that the two sources of geometric errors are independent Gaussian distributions, the margin formalism is then described as $\sqrt{\sigma_{p e}^{2}+\sigma_{o m}^{2}+\cdots}$, where $\sigma_{o m}$ and $\sigma_{p e}$ are the geometric uncertainties due to internal organ motion and patient setup error, respectively. The formation of the PTV using the 
quadratic sum of geometric uncertainties to some extent is more reasonable than that formed with Scenario A.

In scenario C, a "global" safety margin is calculated using the quadratic approach for all sources of the geometric uncertainty, but the presence of Organs at Risk restricts the size of the treatment margins.

ICRU report 50 and its supplementary report 62 were mainly proposed for application to conformal radiotherapy. The limitations of the two reports are summarized as follows.

The ICRU reports carefully describe the GTV, CTV and PTV and their relationship. ICRU 62 also addresses the formation of the planning target volume. Unfortunately, tumour target delineation. uncertainty is not addressed in the margin formalisms. The margin formalism can provide a theoretical basis for developing an optimized PTV. However, in actual cancer clinics, radiation oncologists may make a PTV based on his/her clinical experiences and from studying the literature. In addition, the radiation oncologist also needs to balance between the prescribed dose to the tumour and the tolerance of the OAR when choosing a margin.

In general, geometric treatment uncertainty can be classified into systematic and random uncertainties. It is also well known that the impact of systematic uncertainty on the margin is more significant than that of random uncertainty, but this difference is not addressed in ICRU margin formalisms.

The introduction of new treatment techniques including IMRT, IGRT, Tomotherapy and gated radiotherapy are another challenge for the concept of PTV. A large portion of treatment errors, including positioning error and low-frequency target motion, can now be corrected with image-guided techniques, and thus the IM and SM as suggested in ICRU 50 and 62 are less meaningful. Margin requirements for these techniques must compensate for the residual error (e.g. target deformation and intra-fraction target 
motion). Thus, the concept of the PTV should be re-examined when applied to specific new technologies.

Dose uniformity to the target volumes was emphasized for conformal radiotherapy in ICRU report 50, but this may not be applicable for treatments with new techniques such as IMRT in which any 3D dose distributions can theoretically be achieved within a target volume. Dose uniformity may not be important in these techniques.

\subsection{Review of Geometric Errors in Prostate Target Definition}

Accurate target delineation is a prerequisite for local tumour control and is essential in advanced treatment techniques such as IMRT because of their ability to conform dose to a defined volume. Ideally, the target volume should include all cancerous cells but no healthy cells. A target definition that misses cancerous cells in the target definition could lead to treatment failure. In contrast, encompassing of the healthy tissues in the target definition can cause unnecessary normal tissue complication that should be avoided as much as possible.

Generally speaking, the quality of target delineation for most tumour sites is not well known. It has been shown that the shape and size of the delineated target volume varies significantly depending on many factors ${ }^{[23]}$. These include the types of diagnostic modality, inter- and intra- observer variations, and the tumour site.

The quality of target delineation is limited by the inability to image submillimeter disease with common imaging techniques. ${ }^{[7]}$ Image quality (contrast, distortion, resolution) are known to affect target delineation. A target defined in MRI and PET must be transformed to an appropriate CT scan set in order to be useful for modern treatment planning. This image fusion process can introduce errors. ${ }^{[4,5]}$ A clinical decision regarding the target volume is made after analyzing the comprehensive information from general medical examinations, cancer staging, health status and medical history of the patient. More or 
less, human uncertainties such as knowledge, experience and education may also affect the accuracy and reproducibility of target definition. ${ }^{[24]}$

The delineation of the tumour and the subsequent formation of a target volume that encompasses the entire tumour is one of the key steps in the treatment planning process. However few studies have investigated this uncertainty. One such study of brain tumour delineation reported by Leunens et $\mathrm{al}^{[25]}$ demonstrated a substantial intra- and interobserver volume variation $(25 \% \sim 73 \%)$. In a similar study, Rasch et al ${ }^{[26]}$ measured volume differences in prostate delineation between MRI and CT. These differences were quantified by encompassing and common agreed delineation volumes for both modalities and for multiple observers. The encompassing volume of CT and MRI was found to be larger than the target volume delineated on CT alone for prostate cancer. This was also confirmed by Sannazzari. ${ }^{[5]}$ Rasch also. showed that the variation in the target volume on MRI is less than on CT. Based on his measurements, he concluded that the delineated volume depends more on the imaging modality than on the radiation oncologist. In addition, he demonstrated that there is a large inter-observer variation near the apex of the prostate and a large intra-observer variation at the base near the Seminal Vesicles (SV).

Although CT is the principal modality used to define tumour for most disease sites as well as the basis for the treatment planning process, the contrast on CT images for some tumour sites (e.g. prostate, brain tumour) may not be good enough for accurate target delineation. Instead, MRI can provide better image quality for target contouring of the prostate and brain tumours. The complement of functional MRI and PET images to the anatomically based CT images used in the planning process requires that these images be accurately registered to the planning CT data set. The process of $t$ image fusion brings uncertainty into the target delineation. ${ }^{[5]}$

Table 2.1 summarizes the results of target delineation studies reported in the recent literature for different cancer sites and various modalities. Among these studies, one ${ }^{[26]}$ addressed the significant difference among modalities such as CT, MRI and ultrasound 
imaging, while some emphasized the inter- and intra-observer variability ${ }^{[5,23,25,27-32]}$. It is important to note that in these studies the mean target volume over all delineations from all observers was chosen as the gold standard for target delineation ${ }^{[5]}$ and each delineated target volume was then compared to that gold standard. The use of the group mean to define the true target volume inherently assumes that there is no systematic error and that the average group knowledge is correct. Thus, these data cannot identify the most significant of all errors - systematic error. We have investigated both the reproducibility of the target delineation amongst different oncologists and their accuracy against a true target volume by taking advantage of the Visible Human Project (VHP). The prostate delineation study using the VHP data is described in chapter four.

Table 2.1 Summary of delineation uncertainty studies

\begin{tabular}{||c|c|c|c|c||}
\hline Study & Organ Target & Technique & $\begin{array}{c}\text { Inter-Modality } \\
\text { Uncertainty }\end{array}$ & $\begin{array}{c}\text { Inter-Observer } \\
\text { Uncertainty }\end{array}$ \\
\hline Rasch $^{[26]}$ & Prostate(GTV) & CT/MRI & $+40 \%$ & $15 \%$ in range \\
\hline Cazzaniga $^{[33]}$ & $\begin{array}{c}\text { Prostate+SV } \\
\text { (PTV) }\end{array}$ & CT & - & $36 \%$ (SD) \\
\hline Fiorino $^{[27]}$ & $\begin{array}{c}\text { Prostate } \\
\text { (CV) }\end{array}$ & CT & - & $10 \sim 18 \%$ \\
\hline Sannazzari $^{[5]}$ & $\begin{array}{c}\text { Prostate+SV } \\
\text { (CTV) }\end{array}$ & CT/MRI & $+34 \%$ & - \\
\hline Leunens $^{[2]}$ & Brain (PTV) & CT & - & $25-73 \%$ \\
\hline Hurkmans $^{[28]}$ & Breast & CT & - & $17.5 \%$ (SD) \\
\hline
\end{tabular}

\subsection{Patient Positioning Error}

For external beam radiotherapy, the radiation dose is usually delivered in many fractions to optimize the therapeutic ratio. This technique requires the patient to be positioned up to 40 times on the treatment couch depending on treatment site and treatment protocol and leads to differences in the treatment position between any two fractions. Hurkmans et al ${ }^{[34]}$ defined patient positioning error (or patient setup error) as the difference between the actual and planned positions of the patient anatomy. The planned (sometimes referred to as reference) position is selected on a reference image, either a simulated image or 
digitally reconstructed radiograph (DRR). The selected feature is usually an anatomical structure such as a bone that is close to the treated tumour volume.

In general, there are two types of positioning error. One is rotational positioning error, which is the difference between the actual and planned patient orientation, and another is the $3 \mathrm{D}$ translation positioning error. ${ }^{[34]}$ The rotational positioning error can only be accurately measured using 3D image analysis while the translational component can be detected either by $3 \mathrm{D}$ or $2 \mathrm{D}$ image analysis. Because small rotational errors $\left(<3^{\circ}\right)$ cannot lead to a significant anatomical variation in portal images, its impact on the overall positioning error is usually ignored, particularly for prostate despite it being frequently reported in literature. This leaves only 3-D translational positioning errors in the leftright (LR), Anterior-Posterior (AP) and superior-inferior (SI) directions.

Positioning errors can also be separated into random and systematic errors. Random or so-called inter-fraction positioning errors are deviations among different fractions during the treatment course while systematic errors are the difference between planned and mean treated positions. ${ }^{[35]}$ It has been proven that systematic and random errors are from same distribution. $^{[36]}$

A number of sources for random and systematic positioning errors can be distinguished. These mainly deal with inaccurate alignment of positioning devices (lasers), couch design difference between CT simulators and treatment, patient related error (e.g. skin marker movement, weight change during treatment course), and fixation related error (e.g. supine or prone treatment position with immobilization devices). ${ }^{[37]}$ Another major factor influencing setup error is the accuracy with which the radiation therapists are able to position the patient using the skin marks. This ability is influenced by their experience, training and concentration. These sources of patient setup error were comprehensively reviewed by Hurkmans et al ${ }^{[34]}$ and Booth. ${ }^{[37]}$ 
Table 2.2 Summary of patient setup errors in pelvic sites

\begin{tabular}{|c|c|c|c|c|c|}
\hline Film/EPID/CT & No of Patients & No of Fractions & Directions & $\sigma_{s y s}(\mathrm{~mm})$ & $\sigma_{r a n}(\mathrm{~mm})$ \\
\hline \multirow{3}{*}{$\underset{{\text { (Gayed })^{[38]}}_{\text {EPID }}}{ }$} & \multirow{3}{*}{10} & \multirow{3}{*}{260} & LR & 2.2 & 1.7 \\
\hline & & & SI & 1.0 & 1.2 \\
\hline & & & $\mathrm{AP}$ & 1.4 & 1.9 \\
\hline \multirow{3}{*}{$\underset{\text { (Bijhold) }^{[39]}}{\text { EPID }}$} & \multirow{3}{*}{9} & \multirow{3}{*}{105} & LR & 1.2 & 1.8 \\
\hline & & & SI & 2.3 & 2.1 \\
\hline & & & AP & 2.3 & 1.9 \\
\hline \multirow{3}{*}{ 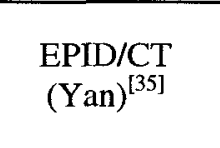 } & \multirow{3}{*}{30} & \multirow{3}{*}{48} & LR & 1.8 & 2.2 \\
\hline & & & SI & 1.9 & 1.8 \\
\hline & & & $\mathrm{AP}$ & 2.9 & 2.2 \\
\hline \multirow{3}{*}{$\begin{array}{c}\text { EPID } \\
(\text { Yan })^{[36]}\end{array}$} & \multirow{3}{*}{25} & \multirow{3}{*}{437} & LR & 3.0 & 3.5 \\
\hline & & & SI & 3.8 & 3.0 \\
\hline & & & $\mathrm{AP}$ & 3.7 & 2.3 \\
\hline \multirow{3}{*}{$\underset{\text { (Bayley) }^{[40]}}{\text { EPID }}$} & \multirow{3}{*}{28} & \multirow{3}{*}{-} & LR & - & - \\
\hline & & & SI & 4.6 & - \\
\hline & & & $\mathrm{AP}$ & 2.5 & - \\
\hline \multirow{3}{*}{$\underset{\text { (Alasti) }^{[41]}}{\text { EPID }}$} & \multirow{3}{*}{33} & \multirow{3}{*}{-} & $\overrightarrow{\mathrm{LR}}$ & & - \\
\hline & & & SI & 1.5 & - \\
\hline & & & $\mathrm{AP}$ & 2.0 & - \\
\hline
\end{tabular}

Table 2.2 summarizes the results of both systematic $\left(\Sigma_{p e}\right)$ and random $\left(\sigma_{p e}\right)$ uncertainties in daily patient positioning error reported in the recent literature. The magnitude of the positioning errors was reported to be orientation dependent. Many studies ${ }^{[36,41]}$ observed the largest overall positioning uncertainty to be in the anteriorposterior direction, but Creutzberg ${ }^{[42]}$ reported the largest uncertainty $\left(\sigma_{p e}=4.2 \mathrm{~mm}\right)$ in the super-inferior direction. As seen in table 2.2, it is commonly agreed that the positioning error was found to be the smallest in the left-right direction among all observed directions. Both Bayley ${ }^{[40]}$ and Alasti ${ }^{[41]}$ have reported the positioning variation in the AP and SI directions because only lateral images were used in their measurements. Based on portal image measurements from 23 patients, Alasti reported that the positioning error ranged from -6.8 to $10.5 \mathrm{~mm}$ and from -6.7 to $7.5 \mathrm{~mm}$ in the AP and SI directions, respectively.

Positioning error is often measured using either films or electronic portal image devices (EPIDs). However, the weakness of these methods is the variable magnification of the 
anatomical structure in the portal images. Due to the nature of $x$-ray projection, the measured positioning error is actually scaled depending on the location of the selected 3$\mathrm{D}$ bone structure with respect to the beam isocentre. ${ }^{[43]}$

\subsection{Internal Organ (Tumour) Motion}

Internal organ motion is a concern for external beam radiotherapy because it can substantially affect the accuracy of the dose delivery to the treated target. Tumour motion has been intensively investigated for different cancer sites. ${ }^{[23,24,39,44-66]}$ Strategies to mitigate tumour motion require knowledge of its cause, orientation, duration, frequency and magnitude. ${ }^{[6]}$ According to Booth, ${ }^{[37]}$ the motion of organs within the body can be approximately classified into random and periodic motion. In general, organs in the upper abdomen such as the lung and liver have been observed to move cyclically, but most organs in the pelvic region have been shown to move randomly. The tumour motion classification also depends on the sampling frequency. For instance, low frequency sampling (e.g. $<0.1 \mathrm{~Hz}$ ) can detect low-frequency target motion such as position variation due to volume fluctuations in adjacent organs, but may not be able to detect the periodic motion due to cyclic breathing $(\sim 0.25 \mathrm{~Hz})$ and cardiac activity $(\sim 1 \mathrm{~Hz})$.

According to frequency, prostate motion can be classified into intra-fraction motion and inter-fraction motion. Intra-fraction prostate motion is defined as the motion of the prostate during a single treatment fraction while inter-fraction prostate motion is defined to be the motion of the prostate between two fractions. ${ }^{[66]}$ There are significant differences in the causes and magnitudes of intra- and inter-fraction motion that have been the focus of many studies. ${ }^{[23,24,39,44-66]}$ In these studies, both inter and intra organ (target) motions were measured to be the temporal target displacement over a period of 10 20mins or one day. As with setup error, the inter-fraction prostate motion can also be classified into systematic and random components. The position of the prostate relative to the bony landmarks in the planning $\mathrm{CT}$ is chosen as a reference ${ }^{[35,67]}$, and inter-fraction deviation from this reference is then measured using CT or portal images during the course of the treatment. The difference between the reference and the average target 
treatment position for patient $i$ is the systematic error and the distribution of the deviation is then defined as the random uncertainty $\sigma_{o m}(i)$. The distribution of the mean difference in a group of the patients is defined as the systematic uncertainty of organ motion, $\Sigma_{o m}$, and the root mean squared average of $\sigma_{o m}(i)$ over the observed patient population is defined as the random component of target motion. ${ }^{[51,68]}$

Intra-fraction prostate motion can be measured by the difference in the prostate position between two frames of a movie clip obtained with an imaging device such as an EPID or ultrasound. ${ }^{[44,69]}$ Because there is no reference in the planning CT for intra-fraction motion, only the random component is usually reported in these studies.

Although many factors contribute to the inter-fraction prostate motion, the physiological functions of the prostate and adjacent organs are identified as major causes of intrafraction prostate motion. ${ }^{[70]}$ The filling of the stomach, rectum and bladder, as well as the action of swallowing can all affect the day-to-day prostate position relative to the reference position. ${ }^{[37,44,66]}$ Haken ${ }^{[71]}$ has demonstrated a correlation between the variation in the prostate position and rectum filling by using lateral radiographs. In this study, the prostate was found to be displaced by more than $5 \mathrm{~mm}$ in 31 of 50 patients when the volume of the rectum varied by $30 \sim 50 \mathrm{~cm}^{3}$. The dominant orientations of this displacement were in the anterior/posterior or superior/inferior directions. In a similar CT-based study, ${ }^{[72]}$ the displacement of the prostate position was observed to be more than $5 \mathrm{~mm}$ in the anterior /posterior direction in 3 of 18 patients in response to bladder filling. 
Table 2.3 Summary of the inter-fraction prostate motion in literature

\begin{tabular}{|c|c|c|c|c|c|}
\hline Study & No of Patient Size & Techniques & Directions & $\Sigma_{o m}(\mathrm{~mm})$ & $\sigma_{o m}(\mathrm{~mm})$ \\
\hline \multirow{3}{*}{ Van Herk ${ }^{[68]}$} & \multirow{3}{*}{11} & \multirow{3}{*}{ Full bladder } & LR & & 0.9 \\
\hline & & & SI & & 1.7 \\
\hline & & & $\mathrm{AP}$ & & 2.7 \\
\hline \multirow{3}{*}{ Zelefsky ${ }^{[65]}$} & \multirow{3}{*}{50} & \multirow{3}{*}{ CT } & LR & 0.6 & 0.5 \\
\hline & & & SI & 2.7 & 2.0 \\
\hline & & & $\mathrm{AP}$ & 2.4 & 1.6 \\
\hline \multirow{3}{*}{$\operatorname{Yan}^{[35]}$} & \multirow{3}{*}{30} & \multirow{3}{*}{$\mathrm{CT}+\mathrm{EPID}$} & LR & 1.8 & 2.2 \\
\hline & & & SI & 1.9 & 1.8 \\
\hline & & & $\mathrm{AP}$ & 2.9 & 2.2 \\
\hline \multirow{3}{*}{$W_{u^{[11]}}$} & \multirow{3}{*}{13} & \multirow{3}{*}{ films } & LR & - & - \\
\hline & & & SI & - & 2.1 \\
\hline & & & $\mathrm{AP}$ & - & 2.3 \\
\hline \multirow{3}{*}{ Dehnad $^{[73]}$} & \multirow{3}{*}{10} & \multirow{3}{*}{ EPID } & LR & - & 2.2 \\
\hline & & & SI & - & 3.2 \\
\hline & & & $\mathrm{AP}$ & - & 2.1 \\
\hline \multirow{3}{*}{ Bayley ${ }^{[40]}$} & \multirow{3}{*}{28} & \multirow{3}{*}{ EPID } & LR & - & - \\
\hline & & & SI & - & 3.5 \\
\hline & & & $\mathrm{AP}$ & - & 3.6 \\
\hline \multirow{3}{*}{ Alasti $^{[41]}$} & \multirow{3}{*}{33} & \multirow{3}{*}{ EPID } & LR & - & - \\
\hline & & & SI & - & 3.3 \\
\hline & & & $\mathrm{AP}$ & - & 5.8 \\
\hline \multirow{3}{*}{ Crook $^{[45]}$} & \multirow{3}{*}{55} & \multirow{3}{*}{ EPID } & LR & 0.5 & 1.5 \\
\hline & & & SI & 5.9 & 5 \\
\hline & & & $\mathrm{AP}$ & 6.0 & 4.1 \\
\hline
\end{tabular}

Table 2.3 summarizes the literature on inter-fraction prostate motion. The inter-fraction motion in these studies was measured either by CT scanning (prior to delivery), electronic portal imaging or conventional films. The number of patients varies from 10 to 50 and the observed fractions range from 4 to 38 . Most studies reported the inter-fraction prostate motion as the standard deviation of the difference between daily prostate position and planned position in three orthogonal directions (AP, LR and SI). The contrast of the prostate is very low on CT and even worse on high-energy portal images. Thus it is almost impossible to directly visualize the prostate with the latter. In order to localize the prostate on portal images, fiducial markers are often implanted into the prostate as surrogates. ${ }^{[23,24,39,41,44-66,74-76]}$ Zelesfsky ${ }^{[65]}$ investigated the motion of the prostate in 50 patients with empty bladders by using four CT scans (initial planning CT and 3 CTs 
during the course of the treatment). The inter-fraction prostate motion was found to be $1.2 \pm 2.9 \mathrm{~mm},-0.5 \pm 3.3 \mathrm{~mm}$ and $-0.6 \pm 0.8 \mathrm{~mm}$ in the AP, SI and LR directions, respectively. Similarly Yan $^{[36]}$ investigated prostate motion by using 18 daily CT scans for 30 patients during their treatments. His results showed that the inter-fraction prostate motion was $2.9 \mathrm{~mm}, 1.9 \mathrm{~mm}, 1.8 \mathrm{~mm}$ for systemic uncertainties and $2.2 \mathrm{~mm}, 1.8 \mathrm{~mm}$ and $2.2 \mathrm{~mm}$ for the random uncertainties in the AP, SI and LR directions, respectively. Other investigators have reported inter-fraction prostate motion using EPIDs and films. ${ }^{\text {[51] }}$ The range of the inter-fraction prostate motion was observed to be $0.5 \sim 6 \mathrm{~mm}$ depending on direction. From these studies (see table 2.3), it can be concluded that the predominant direction of prostate motion is in the anterior/posterior and superior/inferior directions. It is felt that this is because the bone structure in the pelvis is mainly distributed to the left and right of the prostate, leaving little space for soft tissue to move in the lateral direction. Conversely, the lack of bone structure in the anterior and superior/inferior directions of the prostate leaves more space for the prostate and adjacent organs to move along these directions.

In contrast to inter-fraction motion, intra-fraction prostate motion results from high frequency forces including periodic breathing, cardiac function and possibly digestion in the stomach ${ }^{[37]}$. Involuntary patient motion from discomfort or prolonged treatment sessions may also cause intra-fraction target motion. This type of motion might have both random and periodic components depending on the location of the cancer. Vigneault ${ }^{[77]}$ investigated intra-fraction prostate motion using portal images of fiducial markers implanted in the prostate, but no intra-fraction motion was observed in his study. However, Malone et. al. ${ }^{[37,78]}$ reported respiratory associated motions of the prostate for patients immobilized with a thermoplastic shell in a prone position. In his study, the fluoroscopic imaging of implanted fiducial markers was utilized, and the target motion was subsequently measured during simulation. The standard deviation of the maximum prostate displacement in a period of 20 seconds was found to be $2.9 \pm 1.7 \mathrm{~mm}$ and $1.6 \pm 1.1 \mathrm{~mm}$ in the SI and AP directions, respectively. The intra-fraction prostate motion in the lateral direction was unknown. Measurement of intra-fraction prostate motion is very hard to perform because the frequency and magnitude of the motion is usually 
unknown. This means that both sampling length and frequency are very critical in the measurement. If the sampling time is long and the frequency is low, no motion can be detected. This is because the mean tumour displacement is zero over a long period. On the other hand, if the sampling time is short and frequency is high, the detected motion may include some spontaneous motions.

Measurements of inter- and intra-fraction motions in three orthogonal directions (e.g lateral, anterior/posterior and superior/inferior directions) are sufficient for traditional conformal radiotherapy which often applies left/right, and post/ant opposed beams to treat prostate cancer. However, it may not be accurate enough for more sophisticated conformal radiotherapy techniques such as IMRT because more beam directions are applied to achieve a better dose conformity to the target. ${ }^{[79-81]}$ Target motion information in other orientations (e.g. lateral oblique directions) may be useful but has not been reported in the literature. In this thesis, target motion in oblique directions will be emphasized. Details of the oblique target motion are described in chapter five.

\subsection{Reduction Strategies}

Accurate dose delivery to the planned target is a prerequisite for local tumour control. However, geometric uncertainties including target definition, daily setup and organ motion frustrate these efforts. Many strategies have been developed to reduce the impact of treatment uncertainties on the actual absorbed dose. Some investigators ${ }^{[68,82-86]}$ emphasize the use of treatment margins while others ${ }^{[36,87-89]}$ chose daily target repositioning.

\subsubsection{Conventional Target Motion Intervention}

Prostate motion studies ${ }^{[90,91]}$ indicate that the change in daily prostate position is correlated with the volume variation of surrounding organs such as the bladder and rectum. This suggests that the prostate position in the pelvis would be more consistent if the volume of the bladder and rectum were constant during the treatment course ${ }^{[90,91]}$. 
This is the main reason why some treatment protocols require patients to have a full bladder while others require an empty bladder. Traditionally, weekly portal films are taken and compared to simulated images or DRRs. If the displacement of the prostate target is more than a certain distance $(e . g>5 \mathrm{~mm}$ ), the patient is shifted to reflect the target shift from the planned position. However, the efficiency and outcome of this strategy is questionable because the correction for the random component of the treatment error has little effect on the treatment accuracy. ${ }^{[11,42,92]}$

\subsubsection{Target Repositioning}

Systematic error (the treatment error prior to radiation delivery) is one of the largest sources of errors in prostate radiotherapy and is primarily due to a lack of knowledge of the mean treatment target position during the planning process. ${ }^{[36,87-89]}$

Several methods have been utilized to measure and subsequently correct systematic errors. The acquisition of CT images immediately prior to each treatment fraction makes it possible to localize the actual target position at the time of treatment and then realign the radiation beam to the CTV ${ }^{[35,36,87,93-95]}$. The use of linac onboard imaging devices such as EPIDs ${ }^{[39,96]}$ and cone beam $\mathrm{CT}^{[97]}$ have also been reported.

Balter et al ${ }^{[75]}$ investigated the use of fiducial markers implanted in the prostate prior to treatment for two patients and in a phantom. The markers were then imaged using 6 and $15 \mathrm{MV}$ photon beams with an EPID in two orthogonal field directions. The locations of the markers in daily images of patients were determined by a template-matching algorithm. The authors demonstrated that prostate movements can be automatically detected in a phantom within $30 \mathrm{~s}$ of image acquisition. The imaging system was able to detect a displacement in excess of $1 \mathrm{~mm}$ in translation and 1 degree in rotation from its planned position, but the success rate of seed detection was not reported. Poor feature contrast of images acquired through EPI devices is one of the barriers to automatic marker detection and automatic target repositioning ${ }^{[75,96]}$. A variety of algorithms have been proposed to enhance the contrast of fiducial markers in the EPI images and improve 
the success rate of marker detection. Nederveen et $\mathrm{al}^{[98]}$ proposed a marker extraction kernel Using this kernel, the detection success rates for markers of $5 \mathrm{~mm}$ in length by 1.2 and $1.4 \mathrm{~mm}$ in diameter were $95 \%$ and $99 \%$, respectively. $\mathrm{He}$ also reported that the localization accuracy is within $0.6 \mathrm{~mm}$ for all markers. Buck et $\mathrm{al}^{[76]}$ investigated the possibility of applying a 2D Mexican hat function (MHF) for the marker enhancement. The high selectivity of this kernel allows a reliable and precise detection of tungsten markers down to a diameter of $1.5 \mathrm{~mm}$ and a detection success rate higher than $95 \%$ for patients with markers fixed on their skin. The authors also found that the incorporation of the initial marker orientation in the kernel does not significantly improve the detection success rate.

\subsubsection{Margin Optimization}

The overall geometric uncertainties are ultimately mitigated by adding a treatment margin to the tumour target as recommended by ICRU reports 50 and 62. As discussed earlier, how to form this margin as a function of the distribution of setup and organ motion is not well specified in the two reports. Historically, the lack of quantification of the overall treatment errors limited the optimization of the treatment margin. In many cancer centres, the treatment margin is based on the experience of radiation oncologist. Margin optimization becomes more critical in prostate cancer treatments with IMRT and image-guided techniques because they often deliver sharp dose gradients around the planned target while sparing normal tissues. An inappropriate margin could lead to missing the target or to high dose exposure to the adjacent normal tissue. With data from recent studies, ${ }^{[23,36,62,83,85,99]}$ the margin can now be reasonably determined by incorporating overall geometric uncertainties using statistical techniques. These will be reviewed in the next subsections.

\section{- Physical Target Coverage}

As documented in ICRU report 62, separate margins were proposed for patient positioning error and internal target motion. A treatment margin is made by the linear 
addition of the individual uncertainties. This approach is too conservative because the uncertainties are unlikely correlated with each other, so the quadratic sum law should be used. In addition, the organs at risk were not considered in this approach. Several other methods for margin formation were proposed during the $1990 \mathrm{~s}$. These methods ${ }^{[50,100-102]}$ did not separate the systematic and random errors and gave equal weight to all geometric uncertainties. These methods should be re-examined because there is a significant difference between types of errors. ${ }^{[84,103]}$

\section{- Probability CTV Dose Coverage}

The probability CTV dose coverage approach develops a treatment margin incorporating the effect of the overall geometric uncertainty (random and systematic error) on the dose distribution to both the tumour and adjacent normal tissues. The impact of random and systematic errors on the dose distribution to the target is different. Random errors blur the dose distribution, but systematic errors shift the cumulative dose distribution. Therefore, the consequences of systematic errors are more critical than random errors. ${ }^{[68]}$ Effort has been made to develop optimized margin recipes using probability CTV dose coverage approaches by incorporating geometric uncertainties (Van Herk et.al. at the Netherlands Cancer Institute) ${ }^{[103]}$. In this approach, systematic error (or treatment planning error) is defined as the geometric error that enters the process prior to the dose delivery while overall random error is the total geometric error during the dose delivery. By combining overall systematic uncertainty $\Sigma_{T}$ and random uncertainty $\sigma_{T}$, Van Herk used the minimum cumulative CTV dose as the criteria for geometric misses. Based on dose population histograms, he derived a margin recipe to guarantee that $90 \%$ of the patients in the population receive a minimum cumulative CTV dose that is $95 \%$ of the prescribed dose. The margin recipe is given as:

$$
d_{t m}=2.5 \Sigma_{T}+1.64 \sqrt{\sigma_{T}^{2}+\sigma_{p}^{2}}-1.64 \sigma_{p} \approx 2.5 \Sigma_{T}+0.7 \sigma_{T}
$$


where $\sigma_{p}$ is the standard deviation of the beam penumbra, approximately $3.2 \mathrm{~mm}$. Note that this margin was developed based on single-field treatment techniques and is the minimum safe margin to achieve $95 \%$ of the prescribed dose coverage. In another paper, McKenzie ${ }^{[63]}$ suggested that beam configuration (orientation and weight) should also be considered in the margin recipe. A lookup table was created for replacing coefficients (1.64) for the random component of geometric uncertainties as proposed by van Herk. The Stroom group ${ }^{[84,85]}$ also developed a margin recipe. In their approach, a 3-D binary matrix was created with values of 1 inside the CTV and 0 outside. This matrix was then convolved with the overall geometric (systematic and random) uncertainties described by typical normal distributions and resulting in a blurring of the CTV. The coverage probability was defined as the probability for each point to be covered by the CTV. Different CTV coverages and iso-dose curves can be chosen as criteria to form treatment margins. A margin recipe was suggested for prostate cancer by ensuring at least $95 \%$ of the prescribed dose to the CTV volumes for $99 \%$ of patients. This recipe is given as:

$$
d_{t m}=2 \Sigma_{T}+0.7 \sigma_{T}
$$

The advantage of Stroom's margin recipe is that the rotational uncertainty in patient positioning and target motion can be easily incorporated. The margin developed by incorporating dose target coverage is more reasonable than the one developed using physical target coverage because the direct goal of the treatment margin is to achieve the best dose coverage while sparing healthy tissues.

\section{- Biological Endpoint Based Treatment Margin Formation}

In most margin recipes, the treatment margin is determined using criteria such as dose and physical field coverage probability. More recently, both radiobiological effect and the consequence of geometric errors have been emphasized ${ }^{[36,83,86]}$. Yan $^{[36]}$ investigated the effect of geometric errors on prostate cancer treatment in terms of biological effect, but did not discuss treatment margins. Van Herk et al ${ }^{[82]}$ evaluated realistic plans in terms of biological end points such as Tumour Control Probability (TCP) and Equivalent 
Uniform Dose (EUD) in the presence of different geometric errors (systematic, random, translational and rotational) by using Monte Carlo simulations. For a margin of $10 \mathrm{~mm}$ between CTV and PTV in a population of patients, TCP could be reduced by less than $1 \%$ in the presence of geometric uncertainties. When the margin was reduced to $6 \mathrm{~mm}$ to achieve the same TCP, he suggested that the dose must be escalated from 80 Gy to 87 Gy. Based on his simulation, a margin of $2.5 \Sigma+0.7 \sigma-3 \mathrm{~mm}$ is required for at least $90 \%$ of the patients to receive at least $98 \%$ of the EUD. In another paper ${ }^{[83]}$, Van Herk also showed that a margin of $0.4 \mathrm{~mm}$ is required accounting for the effect of fractionation for a random geometric error of $3 \mathrm{~mm}$ for prostate cancer. Song ${ }^{[86]}$ investigated EUD-based margin selection in the presence of setup errors. In this study, a clinical target volume was defined in a cubic phantom and a four-beam conformal plan was designed. For different field sizes and prescribed doses, the margin was varied in two orthogonal directions to examine its impact on the EUD. In addition, a systematic uncertainty of 0,2 , 4, and $6 \mathrm{~mm}$ for setup error and a margin of $10 \mathrm{~mm}$ from CTV to PTV were chosen. Based on simulations and measurements, he concluded that reducing the treatment margin (to $6 \mathrm{~mm}$ ) without reducing the magnitude of the setup error would require dose escalation of 3.5 Gy to avoid a loss in EUD. Van Herk also suggested that tumour cell inhomogeneity and radiosensitivity should also be taken into account when developing a margin in treatment planning. ${ }^{[68]}$ In this case, homogeneous dose distributions to the tumour may not be required and a smaller margin may also be appropriate.

\subsubsection{Intensity Modulated Radiation Therapy (IMRT)}

The primary goal of 3DCRT is to ensure that the spatial distribution of the prescribed dose conforms to the target volume while minimizing the dose to the surrounding normal structures. The delivery of 3DCRT is accomplished with a set of radiation beams that are shaped using a projection of the planning target volume. ${ }^{[43]}$ In conformal radiotherapy, radiation beams often have a uniform fluence across the field. Often critical organs adjacent to the tumour target are not well spared because the dose profile is wide due to the nature of uniform intensity in 3DCRT. This weakness is addressed by IMRT delivery techniques. 
In the past ten years, a new type of treatment planning and dose delivery technique has been developed and has evolved from the concept of conformal radiotherapy. This is the so-called Intensity Modulated Radiation Therapy (IMRT) ${ }^{[104-106]}$. IMRT is an advanced form of conformal radiotherapy that allows the spatial modulation of the intensity of each radiation beam such that each field may have areas of high or low fluence. ${ }^{[8,79]}$ Modulation of both the number of fields and the intensity of radiation within each field increases significantly the number of possible dose distributions that can be delivered to the target. This is the major difference between IMRT and conformal radiotherapy. In IMRT, the spatial intensity modulation can be achieved either by creating a spatial variation of X-rays via collimation or compensation, or by temporally modulating the fluence and varying the temporal modulation in the space domain. ${ }^{[81]}$

In IMRT, intensity modulators such as MLCs or other beam modifiers divide the treatment beam into a set of small beamlets (a small segment of the radiation field). The intensity of each beamlet can vary from 0 to $100 \%$ of the reference dose. IMRT can therefore achieve an abrupt decrease in the dose between the tumor volume and adjacent normal tissues. IMRT has the potential to achieve a higher degree of target conformity and normal tissue sparing than most existing treatment techniques. This is especially the case for target volumes or OAR with complex shapes or concave regions, which represent approximately $30 \%$ of clinical cases. ${ }^{[106]}$

In traditional forward planning, a physician defines the target volume and prescribes the total dose and fraction number. Beam geometry (beam orientations, shape, modifier and weight) is proposed by a planner (a dosimetrist or a physicist), and these are followed by dose calculations. Iterative review of the dose distribution and adjustment of beam properties continues until a plan is made that satisfies the physician's prescription. In forward planning, in order to create a plan which gives the best dose distribution to the target, several plans are generated and compared on the basis of experience and by a process of trial and error. ${ }^{[7]}$ However, in inverse treatment planning, a physician not only defines the target volume but also specifies the optimization criteria. Then, according to 
the specified criteria, an optimization algorithm generates optimal location, shape, and relative beam weightings of the radiation ports as well as beamlet weighting within a port. The optimization criteria in Inverse Treatment Planning play a critical role. Typical optimization criteria include dose distributions to the target volume, the degree of dose heterogeneity within the target volume, areas of exposure avoidance, critical normal tissues, and the relative importance (penalty factor) of the various structures. The optimization criteria are mathematically represented in the form of an objective function. ${ }^{[106]}$ Computer optimization techniques are then performed to determine beam parameters that would best achieve this objective. Currently there are two types of optimization objectives, dose-volume or biologically based criteria. The commonly used dose-volume objective minimizes the variance of the dose relative to the prescribed dose for the target volumes or dose limits for the OARs. ${ }^{[105]}$ Variance is defined as the sum of the squares of the difference between the calculated and prescribed dose. The objective function is the sum of the variance multiplied by a penalty factor associated with an anatomical structure or OAR. For a dose-volume based criterion, the penalty imposed for a failure to achieve the prescribed dose to the target is proportional to the dose difference rather than TCP reduction. The TCP based optimization objective is of current interest in the radiotherapy community, but is limited by large uncertainties in the TCP calculation.

Clinical use of IMRT is motivated by the desire to conform the high-dose region to the target volume without causing normal tissue complication. This is only true if there is no geometric error involved. The logical response to dose escalation in IMRT is that either the geometric error or treatment margin be reduced to avoid high-dose exposure to normal tissues. In IMRT, each segment only treats a portion of the target volume at a time, so there may be significant dosimetric consequences if target motion occurs during the treatment. ${ }^{[107]}$ In addition treatment time with IMRT for prostate cancer is generally longer than with 3DCRT. As a consequence, margin recipes developed for 3DCRT may not be optimized for IMRT techniques. 


\subsubsection{Image Guided Radiation Therapy (IGRT)}

The IMRT technique can achieve a better dose distribution to the target than 3DCRT, but numerous small radiation fields and a longer treatment time ${ }^{[105,106]}$ also increase the difficulty to maintain patient position and manage target motion and subsequently decrease treatment accuracy. Therefore, IMRT generally requires a smaller geometric uncertainty than 3DCRT does. It is commonly agreed that dose escalation with IMRT is challenged by the lack of knowledge of the geometry (relative position and shape) of the target volume and OAR at each treatment fraction. Image guided radiotherapy (IGRT) can significantly improve the accuracy of the target localization. Dose escalation to the target may therefore be achievable with IGRT. In IGRT, an imaging modality is integrated into the linear accelerator and allows for the acquisition of volumetric images of the target and OAR before or during dose delivery. Thus, inter-fraction target motion and patient positioning error can be minimized either by realigning the radiation beam to the target or shifting the patient to the beam. Several approaches ${ }^{[7,75,93,94,97,108,109]}$ have been reported in the literature to accomplish the goal of image-guided therapy. Three configurations are currently being investigated in several institutions. The first approach is the combination of a conventional CT scanner and conventional linac shared with a common treatment couch. ${ }^{[94]}$ The second one is a cone beam kilo-voltage CT orthogonally or laterally mounted to a linear accelerator. ${ }^{[97]}$ The third is the Helical Tomotherapy system. ${ }^{[109]}$ The approach of Tomotherapy represents the most advanced version of the IGRT and is described in next section.

\subsubsection{Helical Tomotherapy System}

The concept of helical tomotherapy was first proposed by Canadian scientist T. R. Mackie $^{[109]}$ in 1993 at the University of Wisconsin and this concept has been developed and commercialized by Tomotherapy Inc. In this concept, the device can best be described as the marriage of a treatment linear accelerator with a helical CT scanner. The beauty of such a device is that it can deliver dose in highly-modulated IMRT mode and at the same time verify radiation delivery. ${ }^{[79]}$ In this system, a $6 \mathrm{MV}$ linear accelerator 
waveguide and a MVCT detector subsystem are mounted on a rotating gantry assembly. They are provided with power via slip-ring technology that also allows data transmission. With this system, the treatment fan beam may also be used to acquire a MVCT image of the patient prior to the treatment. This allows physicians to view a full three-dimensional image of a patient's anatomy and adjust the size, shape and intensity of the radiation beam to the location of the tumor volume before the radiation delivery. During the treatment, a dedicated binary MLC is used to modulate the same fan beam to provide rotating IMRT. The beam rotation is synchronized with the continuous longitudinal movement of the couch through the bore of the gantry, forming a helical beam pattern similar to that seen in a spiral diagnostic CT scanner. The set of binary collimator leaves rapidly transits between open and closed status during radiation delivery. Alternatively, the MLC leaves are retracted to the open state when it is operated as a MVCT. The CT detectors on the Tomotherapy system can also be used to collect exiting photons during the treatment. The measured photon intensities can be back-projected through MVCT scans acquired prior to delivery and a volumetric dose distribution to the virtual anatomy can be computed. The helical Tomotherapy device provides a closed loop for cancer treatment from treatment planning to radiation delivery, and dose verification. Helical Tomotherapy can remove or correct a large portion of geometric uncertainties due to patient positioning error and inter-fraction target motion, but intra-fraction target/patient motion still exists during the treatment. This is one critical issue for Tomotherapy as a small pitch size is applied during the treatment. Another weakness of Tomotherapy is that the acquisition of a MVCT image before each treatment increases the dose (0.5 3 cGy) per fraction to the patient. ${ }^{[79]}$ For a typical prostate cancer treatment (40 fractions), the total dose received from the MVCT image would be from $0.2 \mathrm{~Gy}$ to $1.2 \mathrm{~Gy}$, which represents $0.3 \sim 1.5 \%$ of the prescribed dose (76 Gy). Radiation leakage is another concern in Tomotherapy because the beam-on time is significantly longer than that in 3DCRT. The overall room leakage radiation level was investigated by Balog and coworkers, ${ }^{[110]}$ along with the effect of forward-directed leakage through the beam-collimation system. It was found that a typical treatment could produce up to $1 \%$ dose leakage to tissues remote from the tumour sites. 


\subsubsection{D Conformal Radiotherapy and Radiation Gating}

Respiratory and cardiac associated motions have been found to be the main contributors to intra-fraction target motion and have a significant effect on highly-modulated IMRT, particularly for tumour sites in the thorax and abdomen. ${ }^{[70]}$ The nature of respiratory associated tumour motion is generally cyclic and the period is approximately 4 seconds. Four-dimensional (or tumour tracking) radiotherapy (4DRT) and radiation gating (or gated radiotherapy) have recently been investigated to mitigate respiratory related target motion problems.

Paul Keall ${ }^{[108,111]}$ defined 4DRT as "the explicit inclusion of the temporal change in anatomy during the imaging and delivery of radiation". The goal of 4DRT is to track the tumour position in real time and realign the beam to the target dynamically. There are several steps required in order to achieve 4DRT. The first is to acquire 4DCT for treatment planning. In this step, a patient is scanned using a spiral technique. As the patient is scanned via CT, the respiration signal is simultaneously acquired. Once the images are acquired, they are post processed into individual 3DCT sets according to the respiratory phase at the time each image was acquired. Because the amount of 4DCT images is usually 10 times that of conventional diagnostic CT images, a deformable image registration is applied in 4DRT planning. It allows the volumetric mapping of a 3DCT image set in one respiratory phase and therefore reduces the workload at the time of planning. The CTV and PTV in 4DRT planning are a set of volumes as a function of respiratory phase. Correspondingly, beam portals in 4DRT are also a function of respiratory phase. During radiation delivery, a tracking signal from the tumour position (surrogated by a respiratory signal or marker) of the patient is sent to the computer where it subsequently integrates the tracking signal with the treatment plan. As a tracking signal is already linked to the beam portal in the $4 \mathrm{D}$ plan, once a signal is received, the MLC will be reshaped accordingly. The tumour tracking system in 4DRT can utilize an external system such as an optical motion sensor or spirometer. Potential internal motion tracking systems also include fluoroscopy, ${ }^{[12,112]} \mathrm{EPI},{ }^{[108]} \mathrm{MRI}$ and ultrasound devices ${ }^{[113]}$ for different cancer sites. Tumour tracking 4DRT is challenged by real time tumour 
position measurements and predictions. Additionally, high correlation between target and marker motions is required.

On the other hand, respiratory-gated irradiation is another strategy to mitigate respiratory associated tumour motion during dose delivery. In gated radiotherapy, a patient breathes freely while respiration is monitored. Meanwhile, a signal that is proportional in magnitude to the phase of the respiration cycle can be used to gate the radiation beam. In other words, rather than dynamically reshaping the MLC position to reflect actual tumour position as performed in 4DRT, the beam is fired only during a well-defined inspiration or expiration phase. One critical issue must be solved before the application of radiation gating in cancer treatment. The correlation between the position of the tumour as seen in fluoroscopic images and respiratory induced motion such as chest (or abdomen) motion must be well established. If the correlation relationship is robust, the signal of chest motion can be used to trigger the radiation beam during the treatment. Radiation gating techniques have been investigated in several institutes. ${ }^{[14,115]}$ It is thought to be useful in lung cancer treatment because the relationship between lung motion and inspiration is highly correlated. ${ }^{[46,66,111]}$ Gated radiotherapy is also challenged by complex lung motion and volumetric shape variation. The delay (or phase shift) between lung motion and respiratory signal may be another factor to affect the accuracy of radiation gating. The efficiency for treatment with gated radiotherapy is low because the beam-on time is triggered by the respiratory phase. ${ }^{[111]}$

\subsection{Dose Escalation in Prostate EBRT}

In order to escalate the radiation dose to the target and ultimately improve treatment outcome, the treatment geometric errors associated with prostate cancer radiotherapy must be reduced to avoid an increase in normal tissue complication. Current strategies are to detect the treatment error prior to the treatment and then to correct for it. For most strategies, the cost of the correction for the geometric error is to either increase treatment time or increase the radiation dose to the patient. Table 2.4 summarizes the minimum dose required for target localization with different strategies. ${ }^{[79,97,116]}$ 
Table 2.4 The radiation dose required for image guided radiotherapy

\begin{tabular}{||c|c|}
\hline Techniques & Radiation Dose \\
\hline Low energy x ray & $0.5 \mathrm{cGy}$ \\
\hline Megavolt x ray & $0.5 \sim 2 \mathrm{cGy}$ \\
\hline Diagnostic CT & $1 \sim 4 \mathrm{cGy}$ \\
\hline MV CBCT & $0.5 \sim 10 \mathrm{cGy}$ \\
\hline
\end{tabular}

\subsection{Conclusions}

In this chapter, we have described the treatment process for prostate cancer and associated geometric errors. By reviewing literature, we have found that target delineation, patient positioning error and internal organ motion are three major sources contributing to the overall geometric error. Although many studies have been performed to quantify these errors, the information is still not sufficient to optimize prostate cancer treatment. First, due to the lack of a gold standard for the prostate target, the systematic error is not available for target delineation. Second, although target motion and patient positioning error have been intensively investigated, the orientation, nature and effective strategies are still not well known. Third, the effect of these geometric errors on TCP for different margins, treatment schemes, treatment techniques and their efficiency are still not well determined. Investigating these issues is the aim of this work. 


\section{CHAPTER 3 \\ Electronic Portal Images Devices and Image Analysis Techniques}

\subsection{Overview}

The geometric uncertainties inherent in the process of radiation therapy for prostate cancer presented in this thesis are mostly obtained from analysis of electronic portal images. The data analysis was not straightforward because of poor image quality (e.g. contrast, distortion) and mechanical instability (orientation and translation) of the Electronic Portal Image Device (EPID). Both of these problems were addressed in order to render the images useful for analysis. This chapter provides background information on electronic portal images and describes the methods developed or adopted for image enhancement, image extraction and correction for mechanical instability.

EPID operation and configuration are described in section 3.1. The techniques for contrast enhancement including frame averaging, adaptive contrast enhancement and wavelet technique are described in section 3.2. In section 3.3 the methods used to determine the intercept of the beam isocentre with the EPID detector are described. These include the method of moments, normalized cross correlation and the extraction of interleaf leakage with the Radon transform. Section 3.4 describes the geometric distortion in a clinical portal image and correction method. Section 3.5 addresses mechanical instability of the EPID. Section 3.6 summarizes the accuracy of these methods. 


\subsection{Introduction to the Linac-MLC-EPID Imaging System}

This work was based on a Siemens Digital Mevatron linear accelerator with a 58 leaf double focused MLC (Siemens Oncology Systems, Concord, CA) and a Beamview TI electronic portal imager. The imager consists of a gadolinium oxysulfide screen viewed through a mirror at 90 degrees by a video camera. The imager has a field of view of $26.5 \times 24.8 \mathrm{~cm}$ projected through the plane of the isocentre and a square pixel dimension of $0.508 \mathrm{~mm}$ per side.

\subsubsection{Linac Design and Geometry}

A medical linear accelerator is a key device used for delivering radiation dose to the patient. The accelerator used in this work consists of five major components: an electronics cabinet housing a microwave energy generating source; a rotating gantry containing the accelerator wave guide; an adjustable treatment couch; portal imaging device and operating electronics.

In operation, an electron gun with a tungsten filament produces electrons, which are accelerated with RF power in an waveguide. A bending magnet is used to select the appropriate electron energy and focus these electrons to hit a thin metal target to produce photons. On the way to the patient, the radiation beam is made flat and symmetric by using a flattening filter with quadrant monitor chambers. The dose is monitored using ionization chambers, and the beam is constrained and shaped using the MLC and solid jaw, to cover only the projected target area. During a treatment, the patient is positioned on the couch and the gantry head can be rotated about the patient's superior-inferior axis to achieve optimized entry ports. The Siemens Digital Mevatron linear accelerator produces photons with nominal energies of either 6 or $18 \mathrm{MeV}$, which can be used to treat deep-seated tumours such as prostate cancer. 


\subsubsection{MLC Design and Geometry}

MLCs are commonly used on modern clinical linear accelerators to provide field shaping rather than using Lead or Cerrobend blocks. The Siemens MLC consists of two opposed banks (X1 and X2) of 29 leaves each defining the collimated field in one direction. The central 27 leaves of each bank project to exactly $1 \mathrm{~cm}$ width in the plane of the isocentre, while the outer leaves of each bank project to a width of $6.5 \mathrm{~cm}$. A signature of the Siemens MLC is the inter-leaf leakage, of the order of $1.5 \%$ of the central axis open field dose. The machining tolerances of the MLC leaves are very tight, resulting in a very accurate and reproducible spacing of $10.0 \pm 0.1 \mathrm{~mm}$ in the inter-leaf leakage at isocentre. The positional accuracy of the MLC in its direction of travel (X direction) has a specification of $\pm 2 \mathrm{~mm}$ at isocentre. The collimated field edge is defined in the

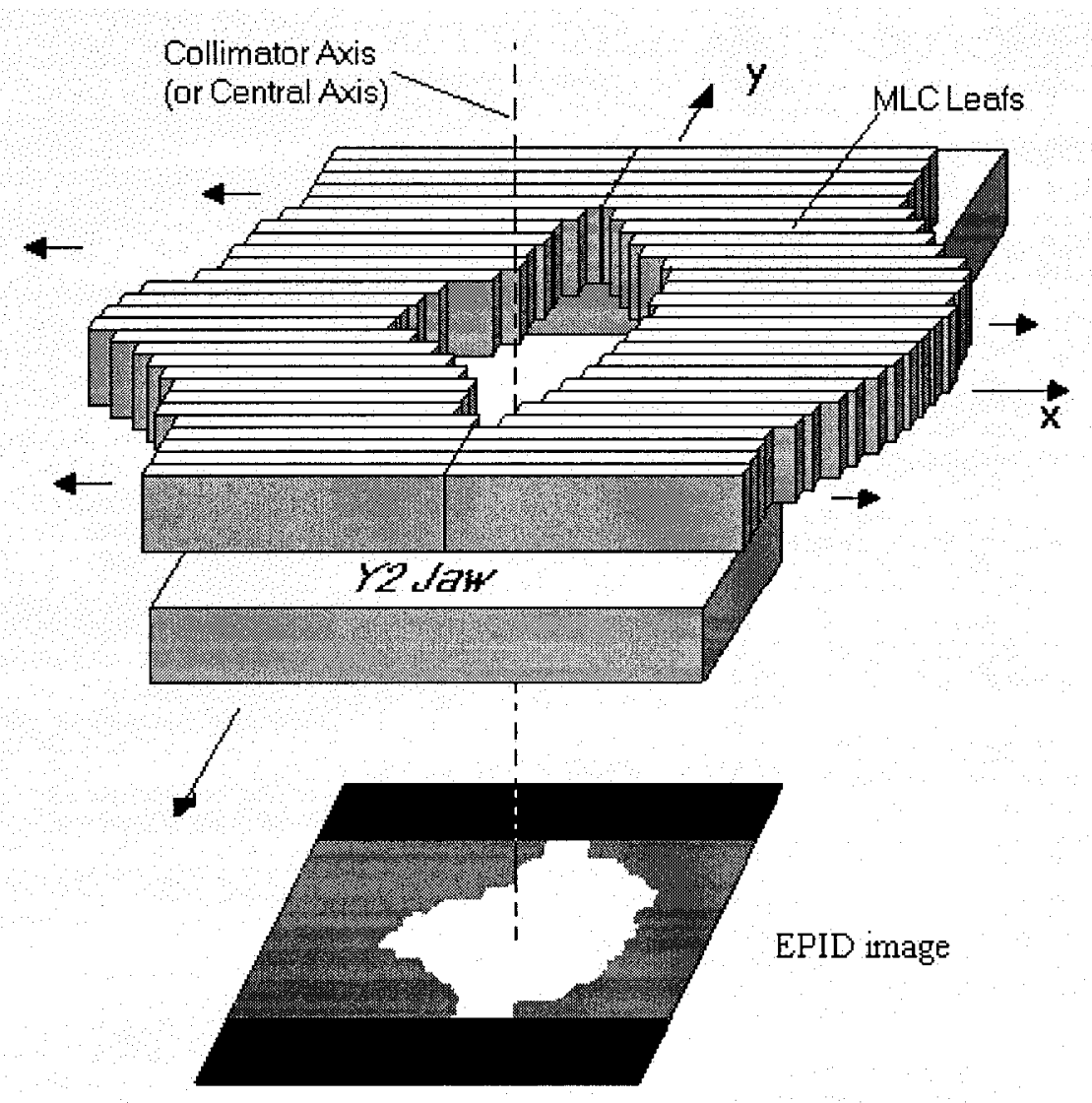

Figure 3.1 MLC and its projection 
perpendicular direction by solid tungsten jaws. Figure 3.1 is an illustration of how an MLC (moving along $\mathrm{X}$ direction) and solid jaw (moving along $\mathrm{Y}$ direction) can shape a beam portal.

\subsubsection{EPID Design and Operation}

In this work we have used the Siemens Beamview TI, illustrated in Figure 3.2. The imager is mounted on the linac gantry on the opposite side of the isocentre from the radiation source. The distance between the radiation source and the detector of the imager is nominally $133 \mathrm{~cm}$. The imager is designed to be folded back against the gantry structure when not in use and deployed as required. It can acquire portal images during treatment delivery at any beam angle and in general does not interfere with patient setup.

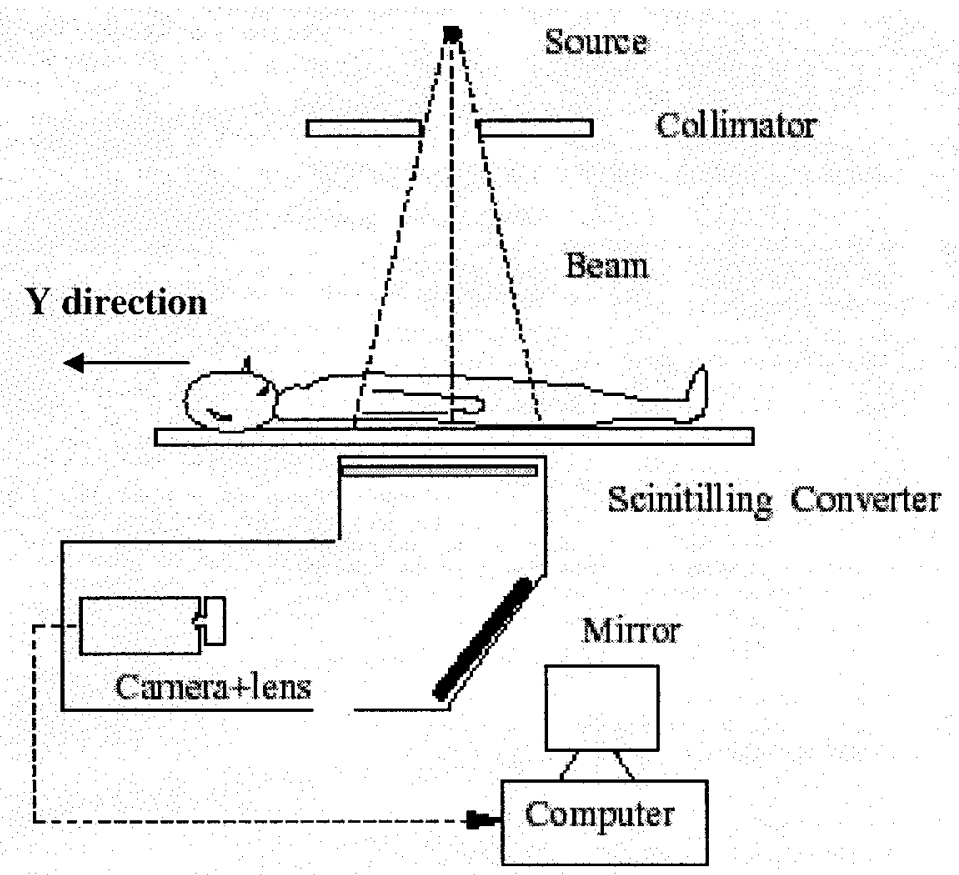

Figure 3.2 Schematic illustration of a camera-based EPID 
The EPID functions by converting $x$-rays to light via an $x$-ray converter, the light is then detected by a charge coupled device (CCD). The $\mathrm{x}$-ray converter consists of a flat metal panel coated with gadolinium oxysulfide phosphor. The metal plate (copper) serves to convert the incident primary mega-volt photons into high-energy electrons, some of which escape into the phosphor. Note that this plate also attenuates the low-energy scatter radiation from the patient, helping to improve image contrast. The phosphor material converts part of the electron energy into the light. This light diffuses through screen, exiting on the mirror side. The mirror then directs this light towards the CCD camera. The use of a mirror in this configuration prevents direct exposure of the camera to highenergy electrons and photons, which would reduce the imager life. The camera converts the light into a video signal, which is sent to other hardware for processing and image display. On average, only $2 \sim 4 \%$ of the incident $x$-rays interact with the imager, ${ }^{[117]}$ resulting in low quantum efficiency.

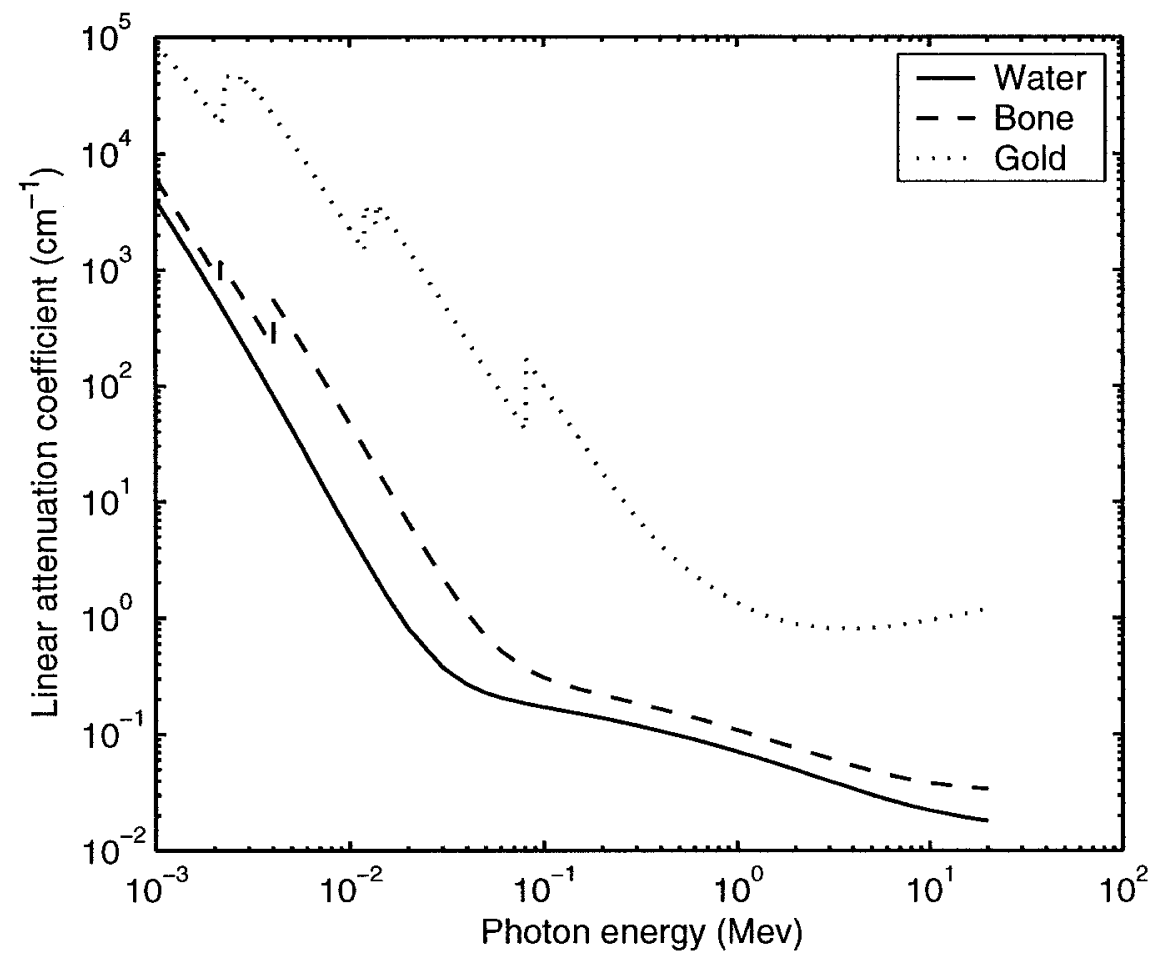

Figure 3.3 Linear attenuation coefficients as a function of photon energy for different compounds ${ }^{[118]}$ 
According to Antonuk, ${ }^{[119]}$ the advantage of a video camera based EPID imager is that it can sense the signal from the entire radiation field simultaneously, providing almost real time images during radiation delivery to a patient. Unfortunately the mirror-lens-camera configuration makes the imaging system relatively bulky and the distance between the imager face and machine isocentre is only $33 \mathrm{~cm}$ making it difficult to image large patients. A major disadvantage of all high energy (therapy) images is the low contrast $^{[117]}$ due primarily to the fact that at therapy energies $(>1 \mathrm{MeV})$ the in-patient attenuation is dominated by Compton interactions as opposed to diagnostic images where the photoelectric process contributes significantly to contrast. As a result, for biological tissues, the main contrast arises from electron density differences, not atomic number differences. There is relatively small variation in the electron density in the human body. Fig 3.3 is a plot of the linear attenuation coefficient as a function of photon energy for water, bone and gold. As can be seen, the linear attenuation coefficient of water and bone is small for the photon energies between 0.1 and $10 \mathrm{MV}$. Note however there is a substantial difference between gold and water. For $6 \mathrm{MV}$ photons, the linear attenuation of gold is approximately 30 times that of water. This large difference makes gold a perfect surrogate for the tumour by implanting it in the patients.

\subsubsection{Information Regions in a EPID Image}

It is necessary in the work described herein to dissect the clinical EPID image into various regions, each containing different types of information. This is shown in Figure 3.4 below, which would be typical of an electronic portal image acquired during prostate cancer treatment. The three distinct information regions are the region containing the radiation portal, a region shielded by the solid jaw and a region shielded by the projection of the MLC leaves. The treatment portal region, shaped by the MLC and the solid jaws is usually located near the central part of the EPID screen and contains anatomical information arising from variations in beam attenuation by bone or air cavities within the patient as well as the tumour (or tumour surrogates). The region shielded by the projection of the MLC leaves contains interleaf radiation leakage which can be used to determine the reference position of the MLC and beam isocentre. The background region 
is the projection area of two solid jaws. This region contains no information for image analysis and is not of interest in this study.

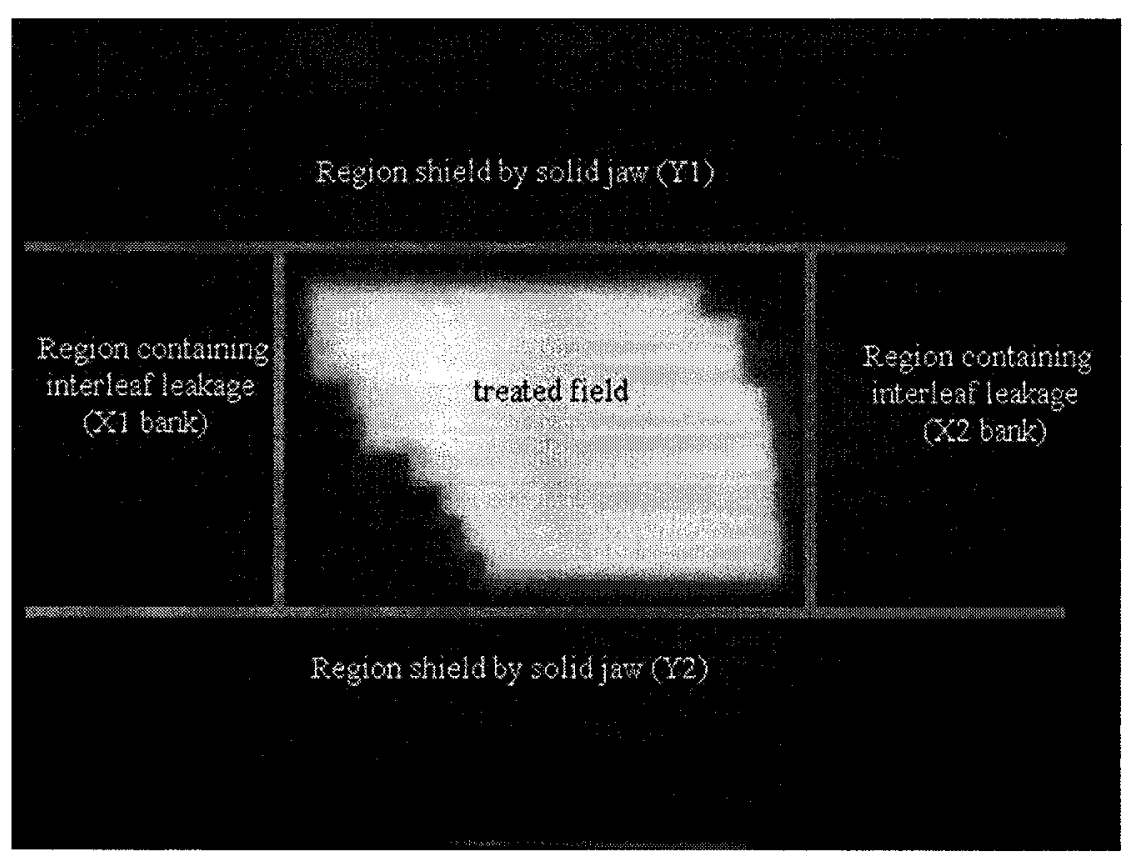

Figure 3.4 diagram of Information segmentations in an EPID portal image

\subsection{Image Enhancement and Processing Tools Used in this Work}

In this work we measure geometric treatment errors using portal images. As such it is necessary to extract the beam isocentre, field borders and orientation, bony landmarks and fiducial markers from the portal image. None of these features can be directly extracted from a portal image due to noise and low contrast of the image. It is therefore necessary to enhance the acquired images before these features can be extracted. This section describes the tools and techniques for the image enhancement that were used in this work.

\subsubsection{Frame Averaging}

Frame averaging ${ }^{[120]}$ is an effective method used to reduce the statistical noise in the image and has been widely used in image processing. In this technique, a series of 
images, all of the same subject and differing only in the independent random noise content are acquired using the Beamview TI imager. These images are then averaged in order to improve the signal-to-noise ratio. The averaging of a series of frame images is given by:

$$
f(x, y)=\frac{1}{N} \sum_{k=1}^{k=N} f_{k}(x, y)
$$

Where $\mathrm{f}$ is the frame averaged image, $\mathrm{k}$ is the frame index of an image. In our approach, we average six consecutive frames of the same treatment field taken daily during radiation delivery, which improves the Signal to Noise ratio by a factor of 2.45 compared to a single frame. However, non-additive image noise cannot be effectively reduced by this technique and as well, frame averaging only works well for images of a static object but introduces blurring of moving targets.

\subsubsection{Contrast Limited Adaptive Histogram Equalization (CLAHE)}

Contrast stretching refers to a simple image enhancement technique that attempts to improve image contrast by stretching a narrow intensity band to the widest allowable intensity range for an image. For the clinical portal images acquired from the Beamview TI imager, the grey levels are either in the range 0 to 30 or 150 to 255 . So the grey scale range changes significantly as a result of intensity stretching. That in turn improves the contrast of the gold seed whose grey scale is in the band of [150 255].

Intensity stretching can improve contrast but not Signal-to-Noise Ratio due to its linear mapping nature. A related process called Contrast Limited Adaptive Histogram Equalization, or CLAHE, ${ }^{[120]}$ modified from intensity stretch, can improve both local contrast and signal-noise ratio. CLAHE was originally developed for medical image processing and has proven to be successful for enhancement of low-contrast images. This algorithm divides an image into contextual regions and applies histogram equalization to each region. As a result, the distribution of image intensity evens out and hidden features 
of the image become visible. Selective feature enhancement is accomplished by first detecting the field edge in a portal image and then processing only regions that lie inside the field edge. Image noise can be reduced while maintaining high spatial frequency content by applying a combination of CLAHE, median filtration and edge sharpening.

\subsubsection{Wavelet Based Contrast Enhancement}

Wavelets are scalable, oscillatory functions that deviate from zero only within a limited spatial regime with an average of zero value. ${ }^{[19]}$ Wavelets may be used to characterize the location of the desired features in an image such as fiducial markers and inter-leaf radiation leakage. ${ }^{[76]}$ In wavelet based image processing techniques, a specified twodimensional wavelet function is convolved with the image, and a correlation map is obtained. The position of the desired feature is determined by locating the peak of the signal strength in the correlation map. Among all wavelet functions, the two-dimensional Mexican Hat Function (MHF) is the most widely applied in the image processing.
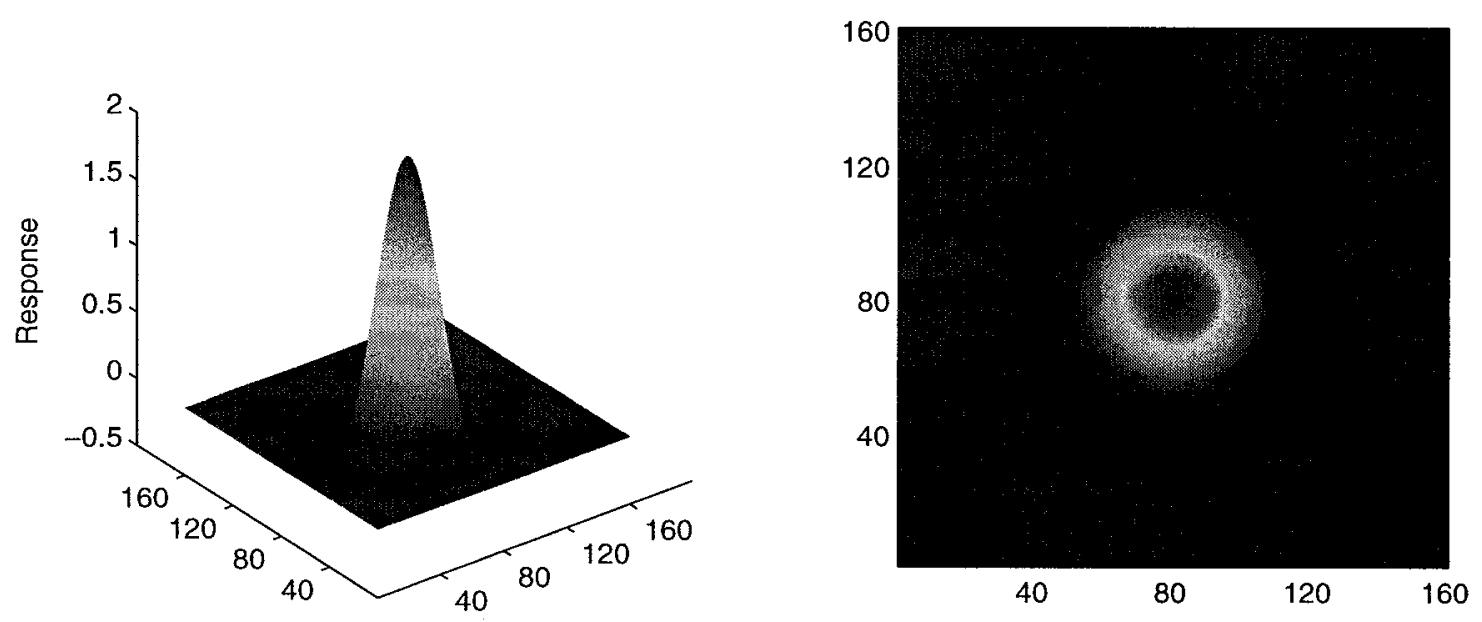

Figure 3.5 Surface and Contour plots of the Mexican Hat Function in position space 

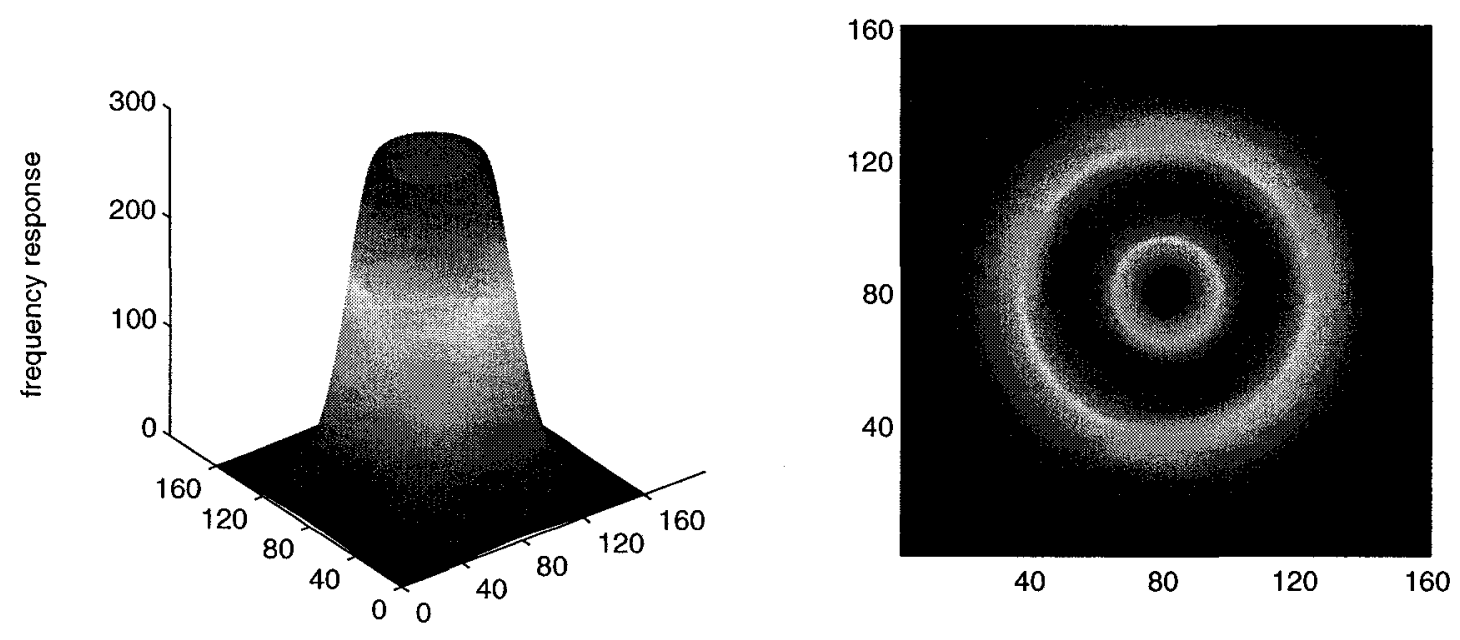

Figure 3.6 Surface and Contour plots of the Mexican Hat Function in frequency space

The form of a general two-dimensional Mexican hat function is defined by:

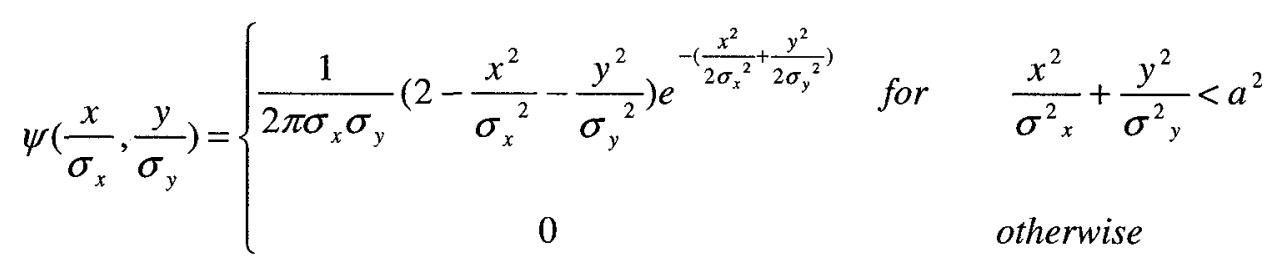

where $\sigma_{x}$ and $\sigma_{y}$ are the dilation or scaling parameters. Figure 3.5 and 3.6 show the surface and contour plots of the 2D MH function in position and frequency space, respectively. It can be seen from the figure that the $\mathrm{MH}$ function has a positive kernel with a shape similar to a conical point spread function, surrounded by a negative annulus. The MH function offers several unique advantages in the gold seed detection in lowcontrast portal images. Firstly, the correlation of a $\mathrm{MH}$ function with constant or linear functions is zero, and thus it effectively suppresses the contribution of the spatially constant or slowly varying signal to the correlation strength. Secondly, the MH's limited extent in both the position and Fourier domain can help to minimize the effects of 
aliasing. This is extremely important if a seed is very close to a high-contrast area such as the portal edge (where background is neither constant nor linear). The ability to scale the kernel to a small size is important since convolution of portal images with a large kernel can allow background noise to contribute to the correlation strength. Scalability is also important since a small size reduces computation time and therefore real time tumour target repositioning becomes possible.

The Fourier transform of the $\mathrm{MH}$ function is given by:

$$
\Psi(u, v)=u v e^{-\left(\frac{u^{2}}{\left.2 \sigma_{u}^{2}+\frac{v^{2}}{2 \sigma_{v}^{2}}\right)}+c\right.}
$$

It can be seen from Figure 3.6 that the MHF is a band limited filter in which high and low frequency noise are suppressed and only signal frequencies close to $\sigma_{u}$ and $\sigma_{v}$ pass allowing SNR to be significantly improved. The selection of the scaling factor represented by $\sigma_{x}$ and $\sigma_{y}$ is very critical. If $\sigma_{x}$ and $\sigma_{y}$ are larger than the actual feature size to be extracted, the feature would be filtered out of an image. On the other hand, if the $\sigma_{x}$ and $\sigma_{y}$ are too small, high-frequency noise would be enhanced which makes it difficult to distinguish real features from high frequency noise. The shape of the implanted fiducial markers is cylindrical with a diameter of $1.1 \mathrm{~mm}$ and a length of 2 mm. However, the shape and size of the marker projection in the portal images are difficult to predict. Furthermore, prostate motion during image acquisitions causes the projection of markers in a portal image to change significantly, making the issue more complicated. 


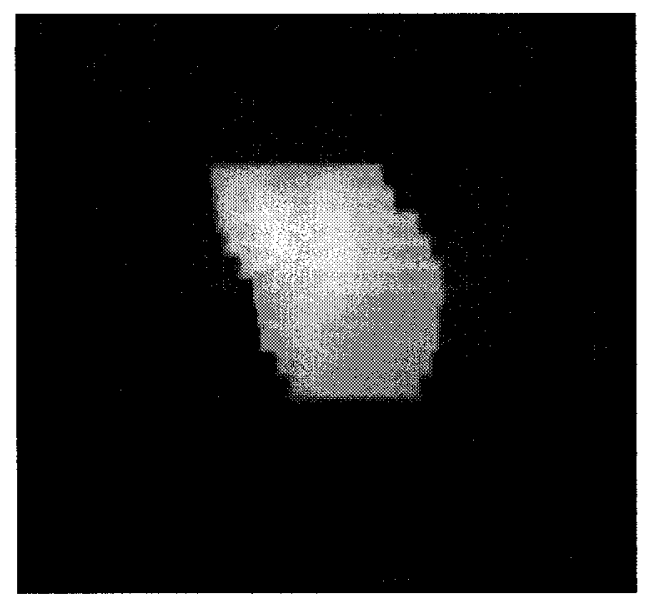

(a)

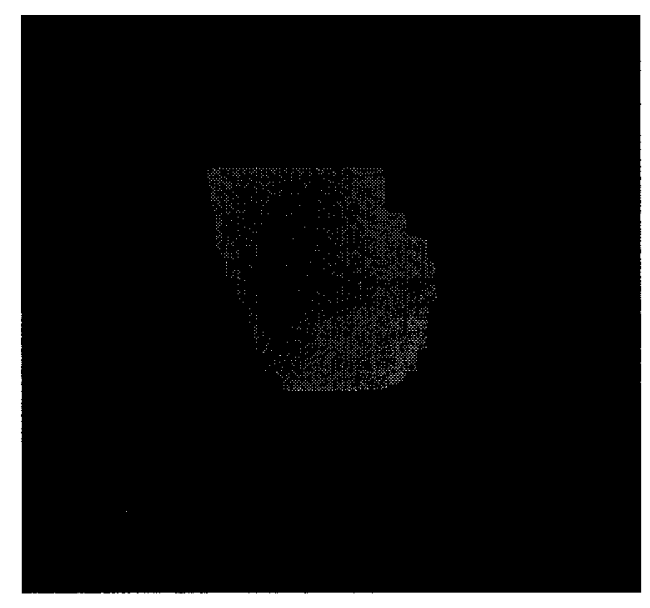

(c)

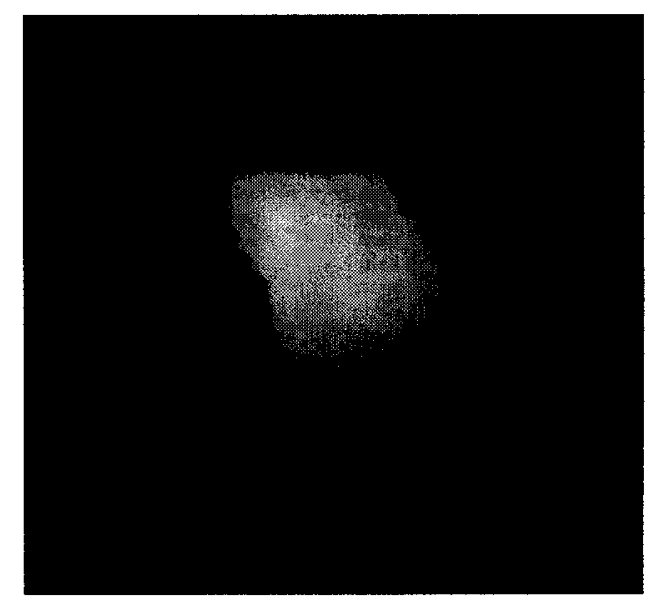

(b)

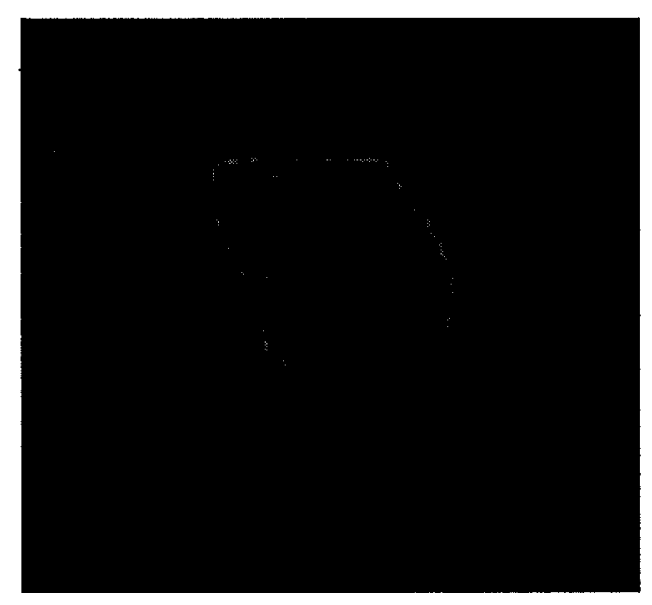

(d)

Figure 3.7 Enhancement of fiducial markers:

(a) Original image (b) intensity stretching (c) CLAHE (d) MHF

As reported by other investigators ${ }^{[76]}$ using the MH function for seed detection, the SNR is not sensitive to the orientation of the seeds in the image. In order to make the selection process simple and also to account for other factors (e.g. motion and seed orientation), a value of $2 \mathrm{~mm}$ was selected for both $\sigma_{x}$ and $\sigma_{y}$ in the seed detection. Similarly, a value of $4 \mathrm{~mm}$ was chosen for bony landmarks. Figure 3.7 illustrates a typical original portal image of implanted markers in pelvis acquired with the Siemens beamview T1 imager (a) 
and its contrast enhanced version using intensity stretching (b), CLAHE (c) and the specified MH function (d).

\subsection{Algorithms for Determining Beam Isocentre and Field Orientation}

Determining target position in the linac frame of reference from electronic portal images requires knowledge of the relationship between the room (or linac) co-ordinate frame and the EPID coordinate system so that the geometric data in a two-dimensional image can be mapped into the 3D treatment room coordinate system. The isocentre is the origin of the treatment room (linac) coordinate system. The intersection of the collimator axis of rotation with the image detector defines the projection of the machine isocentre onto the image plane and acts as the ideal reference point to link the two coordinate systems.

In order to determine the beam isocentre in a portal image, the relationship between the isocentre and beam defining system (solid jaws and MLC leaves) must be defined. An MLC is a computer-controlled beam-shaping device consisting of many pairs of metal leaves or fingers which are usually considered part of the treatment head of a linear accelerator. There are 29 pairs of leaves in the Siemens MLC. All of them except leaves 1 and 29 project to $10 \mathrm{~mm}$ in the plane of the isocentre. The beam isocentre lies at the midpoint of the $15^{\text {th }}$ leaf as specified by the vender. The MLC leaves shape the beam to conform to the PTV shape as projected into the Beam's Eye View (BEV).

The planned portal acts as the template for assessing the delivered portal image. If the edges of a radiation portal can be accurately extracted from the image, the beam isocentre can be determined by matching the extracted edge with the planned edge. However, the edge extraction is limited by many factors including uneven thickness of patient (variation in photon fluence), target motion and MLC positioning performance. Three different methods are used in the determination of beam isocentre. These methods are described in the next few sections. 


\subsubsection{Method of Moments}

In general, the $\mathrm{n}^{\text {th }}(\mathrm{n}=0,1,2,3 \ldots)$ order moment of a random variable $x$ following the distribution $f(x)$ can be defined by ${ }^{[121]}$

$$
m_{x}^{n}=\int_{-\infty}^{+\infty} x^{n} f(x) d x
$$

where $m_{x}^{n}$ is the $\mathrm{n}^{\text {th }}$ order moment for $x$. Similarly, the $\mathrm{n}^{\text {th }}$ order moment of two variables $x$ and $y$ following a distribution $f(x, y)$ can be defined by

$$
m_{x, y}^{n_{x}, n_{y}}=\int_{-\infty}^{+\infty} x^{n_{x}} y^{n_{y}} f(x, y) d x d y
$$

where $n=n_{x}+n_{y}$. If a portal image is assumed to be a continuous function of intensity, the associated moments can be used to determine the beam isocentre ${ }^{[122]}$. The low order $\left(0^{\text {th }}, 1^{\text {st }}\right)$ moment values represent well-known fundamental geometric properties of a distribution or an object. The $0^{\text {th }}$ order moment $m_{x, y}^{0,0}$ of the binary image represents the feature (e.g. radiation portal) area in the given image The two first order moments $\left(m_{x, y}^{0,1}\right.$ and $\left.m_{x, y}^{1,0}\right)$ are used to locate the geometric centre or centroid $\left(x_{c f}, y_{c f}\right)$ of a feature as defined by

$$
\begin{aligned}
& x_{c f}=\frac{\iint x f(x, y) d x d y}{\iint f(x, y) d x d y}=\frac{m_{x, y}^{1,0}}{m_{x, y}^{0,0}}, \\
& y_{c f}=\frac{\iint y f(x, y) d x d y}{\iint f(x, y) d x d y}=\frac{m_{x, y}^{0,1}}{m_{x, y}^{0,0}} .
\end{aligned}
$$


The centroid of a feature in a portal image defines a unique location within the object, which can be used as a reference to describe the beam isocentre within the field of view. The $0^{\text {th }}$ and first order moment are both rotational and translationally invariant. If two objects have the same shape and area, they will have same $0^{\text {th }}$ and first order moment. The portal defined in the TPS can act as the template of the treated area in a portal image. Therefore, the beam isocentre with respect to the centroid in the planning software should be identical to that in the corresponding images. The beam isocentre $\left(x_{i f}, y_{i f}\right)$ in a portal image can then be described as

$$
\left(\begin{array}{c}
x_{i f} \\
y_{i f}
\end{array}\right)=\left(\begin{array}{l}
x_{c f} \\
y_{c f}
\end{array}\right)+\left(\begin{array}{l}
x_{i t} \\
y_{i t}
\end{array}\right)-\left(\begin{array}{l}
x_{c t} \\
y_{c t}
\end{array}\right) .
$$

The coordinate $\left(x_{i t}, y_{i t}\right)$ of the isocentre in the planning template is defined during the treatment planning process. The centroid $\left(x_{c t}, y_{c t}\right)$ of the planned portal in the template image is calculated either using the MLC configuration data or the resulting template from the binary image. Since both the isocentre and the centroid are known in the planned portal, the beam isocentre can then be determined if the centroid of the treated portal can be found. Segmentation of a portal image to its binary type is necessary for the matching between planned and treated portals.

Image segmentation is sensitive to the thresholding level that is selected. An inappropriate threshold can add a large systematic error to the estimate of the beam isocentre. In a radiation therapy department, $50 \%$ of the mid field gray value is often chosen as the threshold value for segmentation of a field portal. Directly applying the $50 \%$ principle in order to segment the field edges can result in errors because the heterogeneity of the patient anatomy can result in a non symmetric beam fluence incident upon the imager. One approach ${ }^{[123]}$ assumes that the treated portal is exactly the same as the one defined in the planning software. The intensity of a pixel within a portal is always higher than background, so the area of the treated portal is forced to be the same as that defined in the TPS by adjusting threshold. For a specified portal image, the 
planned portal area $A_{m l c}$ is given in the corresponding MLC configuration file. Following magnification correction, the area of treated portal is then defined

$$
A_{f}=M_{f}^{2} A_{m l c}
$$

where $A_{f}$ is the area of the treated portal in pixels and $M_{f}$ is the magnification factor. In our case $M_{f}=19.85$ pixel $/ \mathrm{cm}$, determined through phantom experiments. Since the area of a portal image is known, the ratio of areas between the treated portal and an image is given

$$
f_{p}=\frac{A_{f}}{A}
$$

An image histogram as shown in figure 3.8(a) is the greyscale distribution for an image. This histogram can be converted to a cumulative probability function $H(i)$ as shown in Figure 3.8(b) which describes the fraction of pixels in the entire image whose gray value is less than the gray level $i$. The portion of the image which consists of background (outside of the portal) is $\left(1-f_{p}\right)$ which can be specified in terms of $H(i)$ as

$$
H(i)=1-f_{p}=1-\frac{A_{f}}{A} .
$$

The threshold $i$ can be remapped using an inverse transformation of $\left(1-f_{p}\right)$ defined as

$$
i=H^{-1}\left(1-f_{p}\right)
$$

Figure 3.9(a) shows an original portal image with field edges extracted and its binary format after thresholding approach as discussed above. 


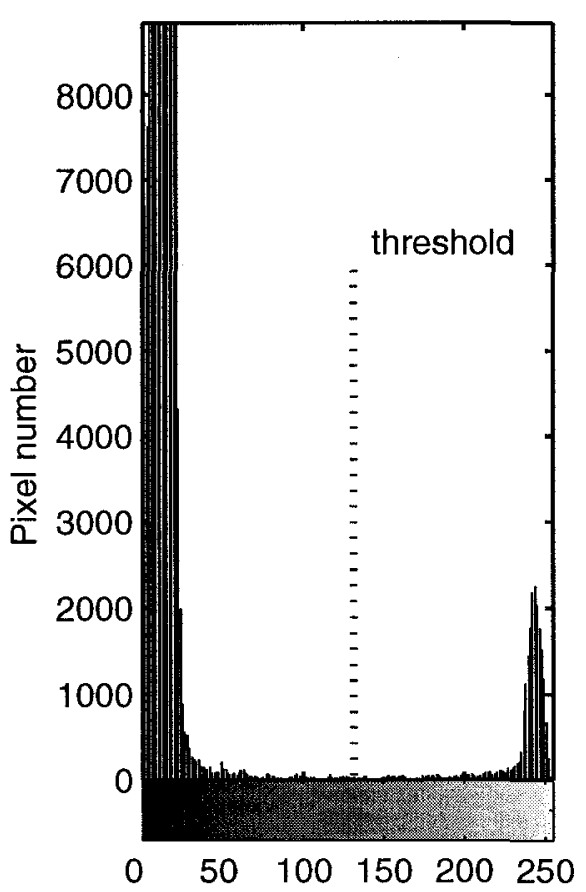

(a)

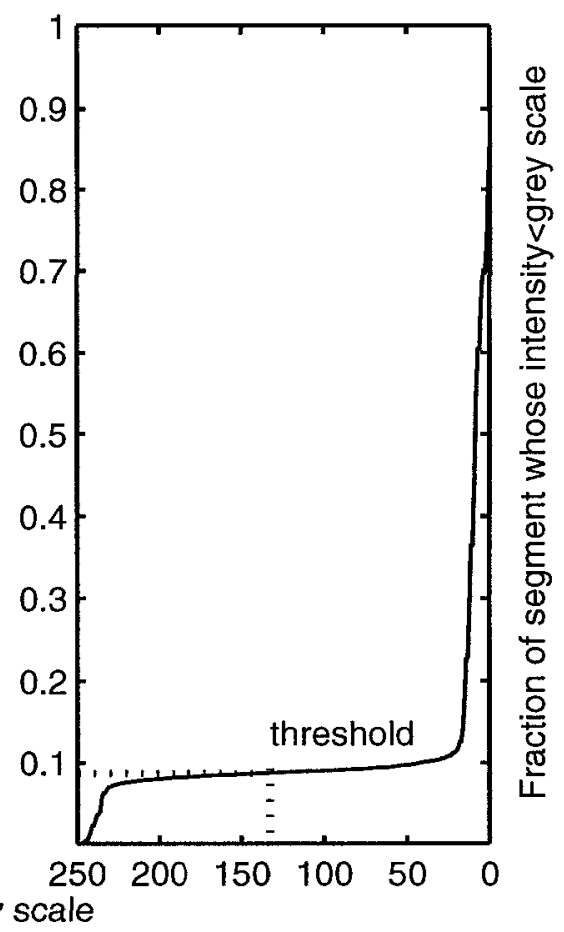

(b)

Figure 3.8 Histogram of a portal image (a) and its cumulative distribution (b)

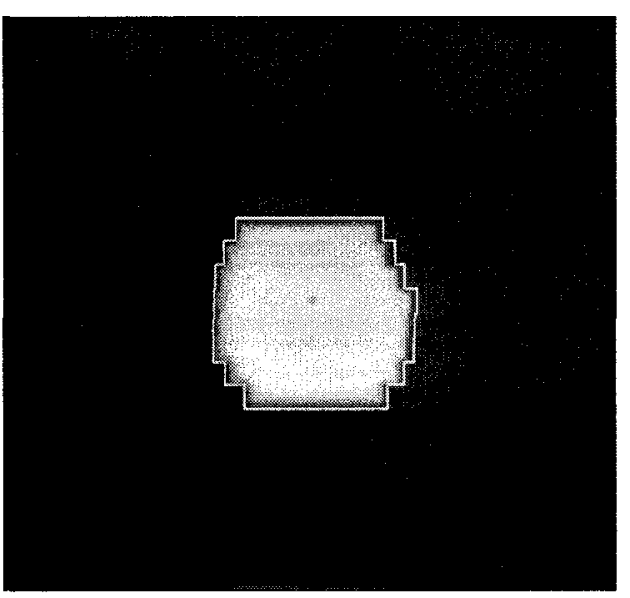

(a)

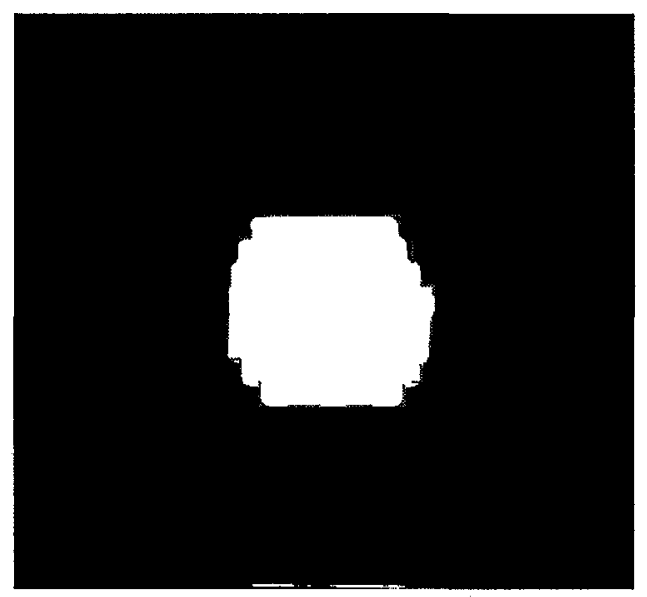

(b)

Figure 3.9 (a) original portal imag (b) binary image after thresholding 


\subsubsection{Normalized Cross Correlation}

Another method by which the planned portal can be "registered" to an image of the delivered portal is by normalized cross correlation. The normalized cross correlation $\mathbf{K}(d)$, of two signals $s_{1}(i)$ and $s_{2}(i)(i=1$ to $n)$, can be defined as

$$
\mathrm{K}(d)=\frac{\sum_{i=1}^{n}\left[s_{1}(i-d)-\overline{s_{1}}\right]\left[s_{2}(i)-\overline{s_{2}}\right]}{\sqrt{\left.\sum_{i=1}^{n}\left[\left(s_{1}(i-d)-\overline{s_{1}}\right)\right] \sum_{i=1}^{n}\left[s_{2}(i)-\overline{s_{2}}\right)\right]}} .
$$

where $\overline{s_{1}}$ and $\overline{s_{2}}$ are the mean values of the two signals. The cross correlation between two signals gives an indication of how similar they are. For a certain $d, \mathbf{K}(d)$ has a peak value where $s_{2}(i-d)$ is most 'similar' to $s_{1}(i)$. The idea of pattern recognition is to cross correlate a template of a known signal with the signal being tested and to search for peaks. In the extreme case, the normalized cross correlation becomes an auto-correlation if two signals are exactly the same. In this work we have determined that a onedimensional normalized cross correlation method can be used to detect the inter-leaf radiation leakage pattern in portal images and subsequently to determine the $Y$ component of the beam isocentre. The performance of the normalized cross correlation technique is limited by the frequency of sampling of a continuous signal because the peak position can only be situated at one location of $\mathrm{K}(d)$. The uncertainty in the peak location is therefore the sampling interval. In order to achieve sub-interval (or sub-pixel) accuracy, peak fitting (or peak centreing) can be applied. In this approach, the peak can be fitted using several points (usually greater than 4) around the peak. Assuming that the cross correlation coefficients around the peak $i$ were found in the interval between $\mathbf{K}$ (i$\mathrm{k})$ to $\mathrm{K}(\mathrm{i}+\mathrm{k})$, the controid of the selected peak can be calculated as 


$$
i^{\prime}=\frac{\sum_{l=k}^{k}(i+l) \mathbf{K}(i+d)}{\sum_{l=k}^{k} \mathbf{K}(i+d)} .
$$

where $i^{\prime}$ is the adjusted new peak location. By using this peak fitting technique, sub-pixel accuracy can be achieved using normalized cross-correlation. Zucker ${ }^{[124]}$ quantified the uncertainty in normalized cross correlation assuming free random noise in the template signal with Gaussian white noise in the test signal as

$$
\sigma_{d_{\max }}^{2}=\frac{1}{N_{T}} \frac{\mathrm{K}\left(d_{\max }\right)}{\mathrm{K}^{\prime \prime}\left(d_{\max }\right)} \frac{1-\mathrm{K}^{2}\left(d_{\max }\right)}{\mathrm{K}^{2}\left(d_{\max }\right)} .
$$

As seen in Eq. 3.15, the standard deviation of peak location can be separated into three factors. The first, $N_{T}$, is the signal sample size which has an inverse relationship to the error as would be expected. Secondly, is $\mathrm{K}\left(d_{\max }\right) / \mathrm{K}^{\prime \prime}\left(d_{\max }\right)$, a normalized measure of the sharpness of the cross-correlation peak which depends weakly on the Signal-Noise Ratio, such that a smaller absolute value of $\mathrm{K}\left(d_{\max }\right) / K^{\prime \prime}\left(d_{\max }\right)$ implies a sharper peak and therefore a smaller error. The third factor, $\frac{1-\mathrm{K}^{2}\left(d_{\max }\right)}{\mathrm{K}^{2}\left(d_{\max }\right)}$, is a measure of the SNR ratio of the cross correlation spectrum, i. e. the ratio between the variance of the signal and that of the noise.

Similarly, assuming that a mask (template) $t(x, y)$ of size $m \times m$ slides over the image $f(x, y)$ of size $n \times n$, the two-dimensional normalized cross correlation coefficient between the template $t(x, y)$ and $f(x, y)$ can be defined as

$$
\mathrm{K}\left(u_{t}, v_{t}\right)=\frac{\sum_{x, y}\left[(f(x, y)-\bar{f}]\left(t\left(x-u_{t}, y-v_{t}\right)-\bar{t}\right]\right.}{\sqrt{\sum_{x, y}\left[( f ( x , y ) - \overline { f } ] ^ { 2 } \sum _ { x , y } \left[(t(x, y)-\bar{t}]^{2}\right.\right.}},
$$


where $\bar{t}$ is the mean grey scale of the template image and $\bar{f}$ is the mean grey scale of the test image $f$.

A planning portal with respect to isocentre is often defined in the MLC configuration file of planning software. This planning portal can be utilized to create a template image for normalized cross correlation. An image matrix is created for the minimum encompassing area of the planning portal region with a $1 \mathrm{~cm}$ additional margin. Note that the spatial resolution of the matrix is set the same as that of a clinical image. The pixel values within the planning portal are set to 1 while the pixel values outside the planning portal are set to 0 . The resulting binary matrix is then defined as our template image $t$. The location of the isocentre in the template image can be theoretically calculated from the planning software. As an example, Figure 3.10 shows the map of the normalized cross correlation between a generated template image $t$ and a clinical portal image $f$. The location of the global peak indicates where the pattern (e.g. radiation portal) is most similar to the template. Applying the peak finding algorithm to determine the location of the peak in the cross correlation map, the displacement between the template image and test clinical image can be determined as:

$$
\left(\begin{array}{c}
\Delta x \\
\Delta y
\end{array}\right)=\left(\begin{array}{l}
x_{\max }^{c} \\
y_{\max }^{c}
\end{array}\right)-\left(\begin{array}{c}
N_{x}^{t} \\
N_{y}^{t}
\end{array}\right)
$$

where $\Delta x$ and $\Delta y$ are the translations between template and the portal image in the $\mathrm{X}$ and $\mathrm{Y}$ directions respectively. The point $\left(x_{\max }^{c}, y_{\max }^{c}\right)$ is the location of the global peak in the normalized cross correlation map and $N_{x}^{t}$ and $N_{y}^{t}$ are the size of the template image in X and $\mathrm{Y}$ directions, respectively. The beam isocentre in the portal image can then be determined by: 


$$
\left(\begin{array}{l}
x_{i f} \\
y_{i f}
\end{array}\right)=\left(\begin{array}{l}
x_{i t} \\
y_{i t}
\end{array}\right)+\left(\begin{array}{l}
\Delta x \\
\Delta y
\end{array}\right)
$$

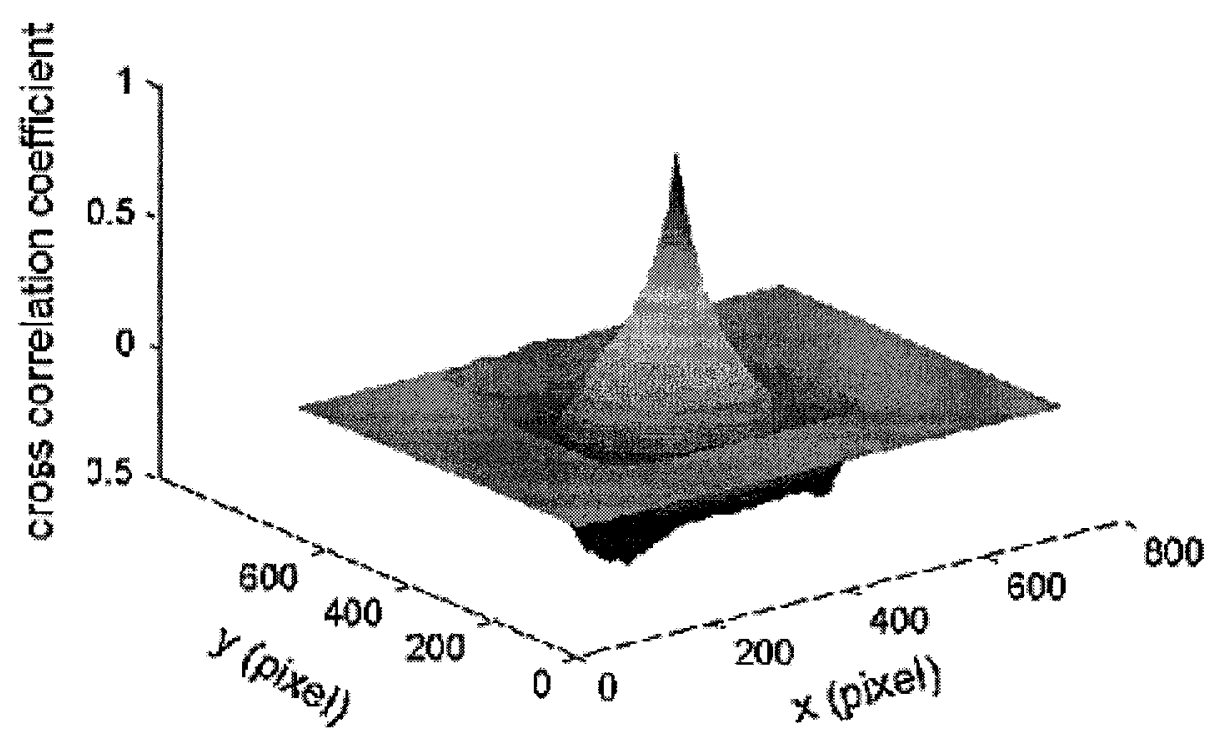

Figure 3.10 Normalized cross-correlation between a template and a portal image peak location represents the best match between two images

The cross-correlation tool is robust to image noise and very precise in offset (or shift) estimation, but it is not invariant with respect to rotation and is sensitive to perspective distortion. Clinically it is possible that the orientation of the portal image is rotated from what it was planned making the use of cross correlation suspect. It should also be noted that cross correlation is computationally intensive) which may yield it impractical for use in real time target positioning in a clinical setting. 


\subsubsection{Line Pattern Detection Using the Radon Transform (Extraction of Interleaf Leakage)}

Multi-Leaf Collimators (MLCs) are commonly used on modern clinical linear accelerators to provide field shaping rather than using Lead or Cerrobend blocks. It is common that the adjacent leaves of an MLC have a small gap between them allowing a small amount of photon transmission (up to $2 \%$ ). ${ }^{[118]}$ When patients are imaged during radiation therapy, this interleaf leakage is integrated as a part of the portal image. Using image processing tools such as the Radon transform and cross correlation techniques, the inter-leaf leakage can be extracted and then utilized to accurately determine the spatial co-ordinates of imaged objects with respect to the beam isocentre.

\subsubsection{Radon Transform}

The Radon transform has been widely used in medical image reconstruction for modalities such as CT and MRI. Recently it has been applied to the field of image processing, particularly pattern recognition. The Radon transform is able to transform line patterns in the image domain into dot patterns in the Radon domain making it possible to easily recognize line parameters. ${ }^{[125]}$

In general, a Radon transform, or the ray-integral of a portal image $f(x, y)$ along direction $\theta$, is defined by the following expression:

$$
g_{\theta}(s)=\iint f(x, y) \delta(s-x \cos \theta-y \sin \theta) d x d y
$$

where $\delta$ is the Dirac delta function, $f(x, y)$ is the image intensity at location $(\mathrm{x}, \mathrm{y})$, and $r=x \cos \theta+y \sin \theta$ is the perpendicular distance from the image origin (image centre) to the straight line along which the ray-integral is evaluated. Eq. 3.19 can also be regarded as the linear intensity integration of image $f$ along a particular angle $\theta+\pi / 2$. Note 
that Eq. 3.19 treats the image as continuous function. In practice a discrete Radon transform is used as well as nearest neighbour image interpolation.

Figure 3.11 illustrates the Radon transform of an oblique and a horizontal line. On the left (Figure 3.11 (a)) is the original simulated images and on the right (Figure 3.11 (b) the corresponding Radon transform. Note that in the Radon domain, a line in the image domain yields a peak (hotspot) located at a corresponding line parameter position (orientation and radius in the Radon domain). The size of the hotspot depends on the line width while the brightness (or sharpness) of the dot depends on the length and contrast of a line pattern in the original image.

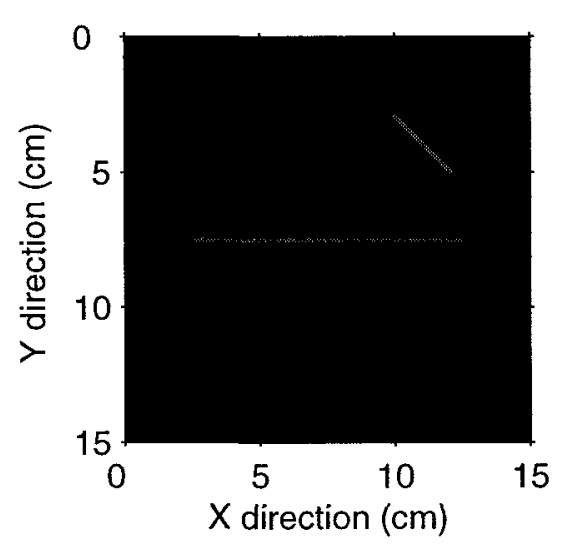

(a)

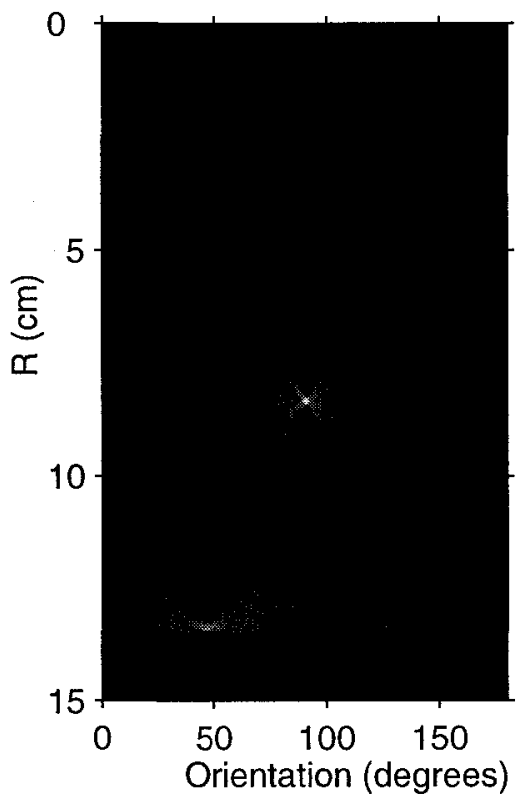

(b)

Figure 3.11 Radon transform (b) of the line patterns in (a)

Two straight lines with different orientation in (a) can be easily defined in its Radon

Domain representing two dots. The location of the dot describes the orientation and position of the specified line in its image domain, the brightness of the dot is determined by the contrast and length of the line 
A very powerful property of the Radon transform is its ability to detect line signals embedded in very noisy images. It is this property that has been utilized to detect and extract interleaf radiation leakage from noisy portal images as long as the length of the leakage interleaf leakage line is sufficiently long despite its low contrast. Figure 3.12(b) is a Sinographic plot of the radon transform of a portion of a portal image containing inter-leaf leakage as shown in Figure 3.12(a). As can be seen, the interleaf leakage lines in the original image are very faint and difficult to detect by eye, while the bright spots representing the orientation and location of the interleaf can be clearly seen in Radon space.

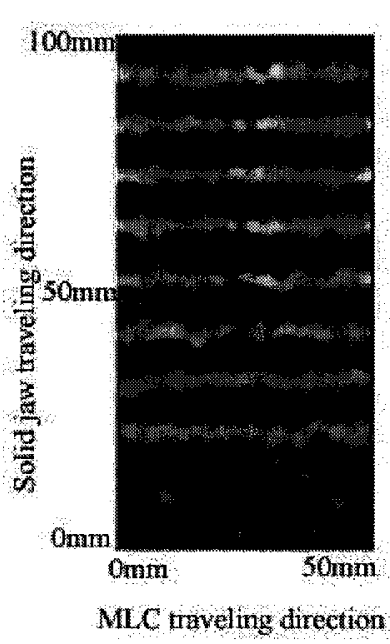

(a)

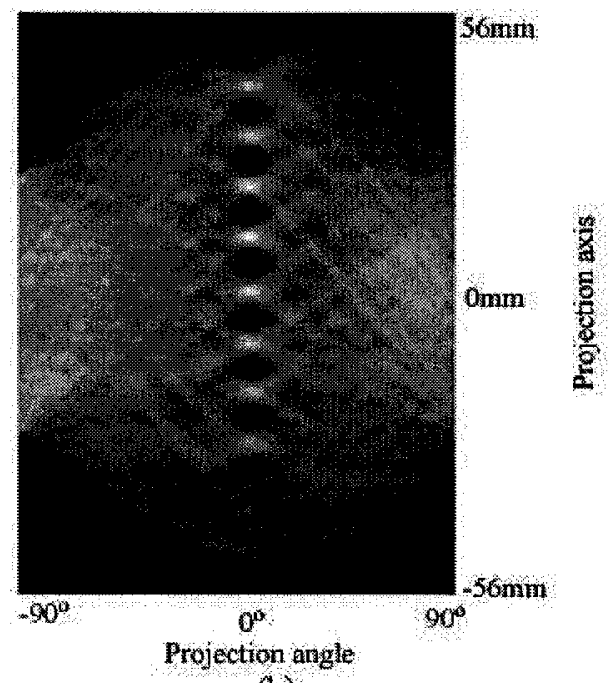

(b)

Figure 3.12 (a) Portal image containing inter-leaf leakage and (b) the corresponding sinographic plot from the Radon transform 


\subsubsection{Determination of Orientation}

As an example, Figure 3.13 shows the Radon transform $g_{\theta}(r)$ of the image $f$ at $0^{\circ}$ (parallel to the inter-leaf leakage) and $90^{\circ}$ (perpendicular to inter-leaf leakage) to the direction of motion of the MLC leaves. The Radon transform can be thought of as the sum of all intensity values along the integration path, thus when the path of integration is parallel and coincident with the leakage radiation the signal (sum) will be significant. When the integration path is parallel but not coincident with the leakage radiation the signal will be weak and similarly when the integration path is perpendicular to the leakage radiation there will be very little spatial variation in the signal. Thus, $g_{\theta}(r)$ can be described as a stationary signal, which is modulated by inter-leaf radiation leakage and the period of the signal is then the width of a single leaf along the line of integration at angle $\theta$. The signal contrast of inter-leaf radiation leakage can be defined as:

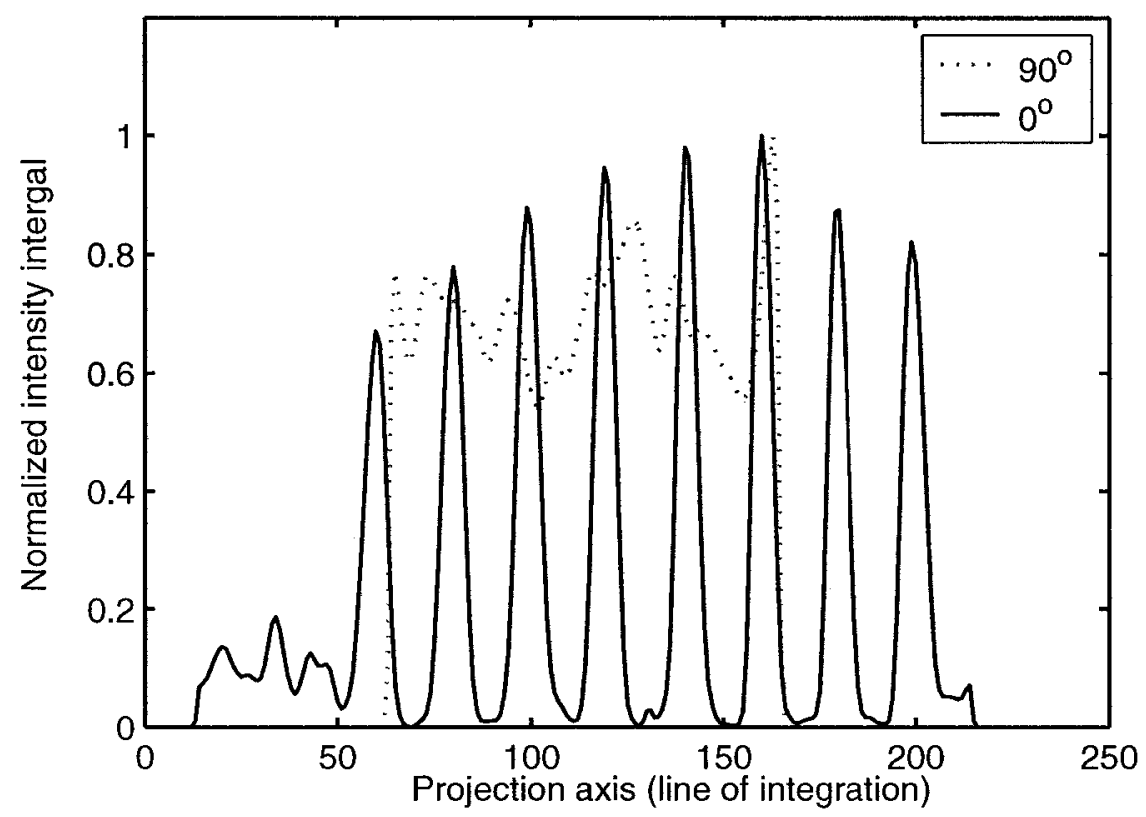

Figure 3.13 Showing the Radon transform of interleaf radiation in the horizontal and vertical directions 


$$
C(\theta)=\frac{100 \times\left(s_{\theta}^{\max }-s_{\theta}^{\min }\right)}{2 s_{\theta}^{\max }}
$$

where $s_{\theta}^{\max }$ and $s_{\theta}^{\min }$ are maximum and minimum values of signal for a particular angle. Thus the contrast of the inter-leaf leakage as a function of integration angle $\theta$ can be used as a means of determining leaf (and hence collimator) orientation in a portal image such that $C(\theta)$ is a maximum when $\theta$ is parallel to the leaf orientation $\theta_{\text {leaf }}$.

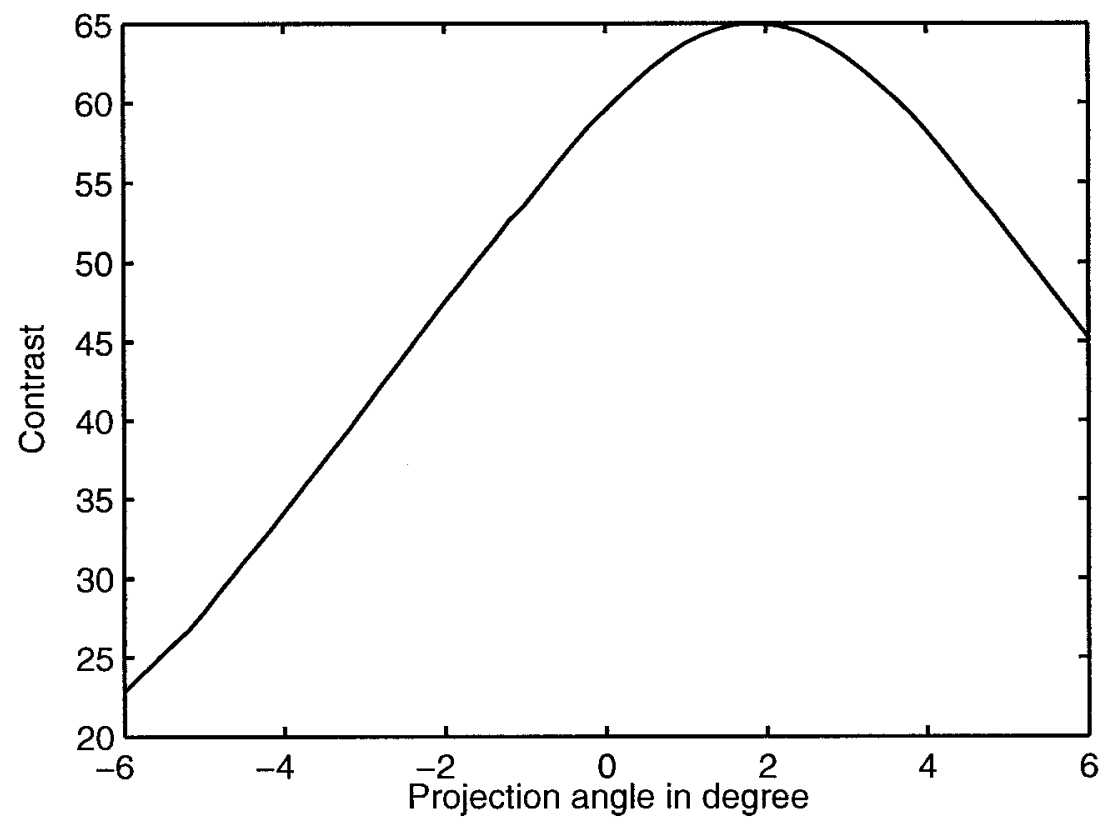

Figure 3.14 Contrast of inter-leaf leakage as a function of projection angle

Figure 3.14 is a plot of contrast $C(\theta)$ of inter-leaf leakage as a function of projection angle $\theta$ from $-5^{\circ}$ to $5^{\circ}$ for a test image with an intentionally set and independently measured collimator angle of $2^{\circ}$. As can be seen in the figure, $C(\theta)$ reaches a maximum at a projection angle of $2^{\circ}$. Thus as can be seen, the Radon transform can be used to determine leaf orientation in a portal image, which in turn dictates the collimator rotation 
relative to the imaging chain. Assuming the collimator angle is well known then image to image variations in the imaging chain orientation $\left(\theta_{i m}\right)$ are given by

$$
\theta_{\text {im }}=\theta_{\text {leaf }}-\theta_{\text {col }}
$$

This method was tested by acquiring images for five known collimator angles $(0,1,2,3$, and $4^{\circ}$ ) with a Siemens Beamview TI (Concord, CA) portal imager. The leaf orientation in the images was determined by the Radon transform method for three conditions: single image leakage through the X1 bank only, single leakage through both the X1 and X2 banks and finally average leakage through both the X1 and X2 banks from six consecutive image frames.

\subsubsection{Determination of Beam Isocentre}

The Radon transform analysis of the inter-leaf leakage can also be used to accurately determine the $\mathrm{Y}$ component of the intersection of the beam axis with the portal imager. The algorithm is described as follows.

The Radon transform $g_{0}(r)$ parallel to the MLC leaves will produce a signal with high contrast with a peak at locations ( $y_{i}^{\text {peak }}$ ) where the inter-leaf leakage is maximum. This signal will have a period of distance $w$ which is the projection width of an individual leaf. The $\mathrm{Y}$ component of beam isocentre $y_{i f}$ should be exactly (assuming very high manufacturing tolerance) in the middle of the $15^{\text {th }}$ leaf for a 58 leaf Siemens MLC. That is also to say, $y_{i f}$ is a half leaf away from $y_{14}^{\text {peak }}$ and $y_{15}^{\text {peak }}$. Locations of leakage for $y_{i}^{\text {peak }}$ can then be determined by:

$$
y_{i}^{\text {peak }}= \begin{cases}y_{i f}+(i-15.5) w & i>15 \\ y_{i f}+(i-14.5) w & i<15\end{cases}
$$


where $i$ is the leaf index. The least square fit of the measured $y_{i}^{\text {peak }}$ to Eq. 3.22 allows an accurate determination of $y_{i f}$. Having determined the collimator axis of rotation in this direction, the position in the orthogonal direction can be determined by several methods. The most accurate and simplest is to rotate the collimator by $90^{\circ}$ and repeat the method above. This is not always possible, particularly in the clinical environment. An alternative method is as follows. As $y_{i f}$ is predetermined with interleaf radiation leakage, a strip containing exact half width of the leaf $i$ can then be cropped from the image that interleaf leakage was obtained. For each extracted strip $i$, the actual measured left and right leaf edges $\left(x_{i, 1}^{m}, x_{i, 2}^{m}\right)$ can be independently determined through local thresholding. Additionally, the expected edges of leaf $i$ can also be calculated as

$$
x_{i, 1}^{e}=x_{i f}+d_{i, 1}^{p} \text { and } x_{i, 2}^{e}=x_{i f}+d_{i, 2}^{p}
$$

where the leaf position $d_{i, 1}^{p}$ and $d_{i, 2}^{p}$ in two banks are assigned by the planning software, respectively. If the nature of the MLC positioning error is random, $x_{i f}$ can then be determined by using variance minimization for the selected leaves, given by:

$$
\mathcal{E}=\sum_{i}\left\lfloor\left(x_{i f}-d_{i, 1}^{p}-x_{i, 1}^{m}\right)^{2}+\left(x_{i f}+d_{i, 2}^{p}-x_{i, 2}^{m}\right)^{2}\right]
$$

The advantage of the local thresholding is that it avoids the impact of the global nonuniformity in intensity in portal images.

These methods of finding the beam isocentre in the EPID image were tested using a calibrated isocentre jig consisting of a $0.5 \mathrm{~mm}$ sphere placed on the collimator axis of rotation. By independent projection / rotation analysis the $0.5 \mathrm{~mm}$ steel sphere is known to be within $0.1 \mathrm{~mm}$ of the collimator axis of rotation. Using the projection of this sphere onto the EPID image as a reference we tested the methods described above using 22 different MLC shaped fields and the results are described in section 3.6 


\subsection{Geometric Distortion and Correction}

Geometric distortion of the EPID image arises in the imaging chain from distortions in the lens, mechanical distortions in the mirror and from misalignment of the mirror. Correcting the image for this geometric distortion requires a mapping of the EPID image into a flat Cartesian space. In this study, the geometric distortion of the EPID was measured by taking advantage of unique properties of the MLC. From these measurements an image-to-physical space transform is developed to correct for the geometric distortion.

The spatial reference point is the radiation beam isocentre, which is defined by the axis of rotation of the collimator system. In order to find the intercept of the collimator axis of rotation with the image plane multiple images were taken with all MLC leaves fully open except the middle leaf (\#15) of one bank, which was positioned $5 \mathrm{~cm}$ across nominal beam centre. 18 successive images were then acquired, one every 20 degrees of collimator rotation, and superimposed. A surface plot of the superposition of 18 images is shown in the Figure 3.15. Note that this is in fact the complement of the original image superposition. The hotspot was formed by the superposition of multiple images because by definition the position of the collimator central axis is rotationally invariant and is consistently projected into a fixed pixel in the composite image while exposures to other pixels are generally averaged. The location of hot spot $\left(x_{i f}, y_{i f}\right)$ in the composite image can be accurately determined by peak thresholding and accurately defines the beam isocentre. 


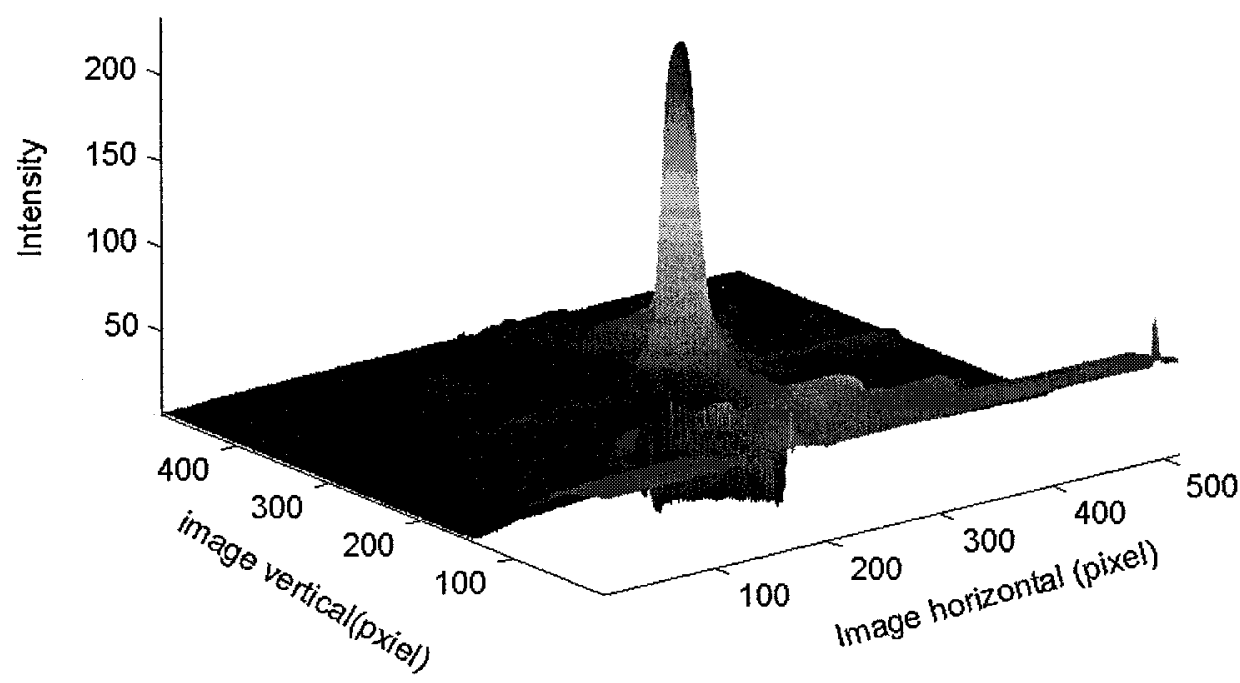

Fig 3.15 The superposition of 18 images of leaf 15 at 18 collimator angles

The geometric distortion of the entire imaging chain was measured using a well-defined geometric pattern created in the image plane by the superposition of two images. Each is an image of the MLC with all of the even numbered leaf pairs fully closed and the adjacent odd number leaf pairs fully open. The two images of the superposition are identical but taken at collimator angles exactly 90 degrees apart (collimator $0^{\circ}$ and $90^{\circ}$ ). The resulting composite image is shown in Figure 3.16. In the Beam's eye view this grid should be square by definition. The composite image was made binary by local thresholding to produce a white $(2 \mathrm{~cm} \times 2 \mathrm{~cm})$ grid. The centroid $\left\{x_{f}(i), y_{f}(j)\right\}$ of each bright square is then determined by using the normalized cross-correlation between binary composite image and a generated $2 \mathrm{~cm} \times 2 \mathrm{~cm}$ white block. The normalized cross correlation was described in section 3.3.2. 


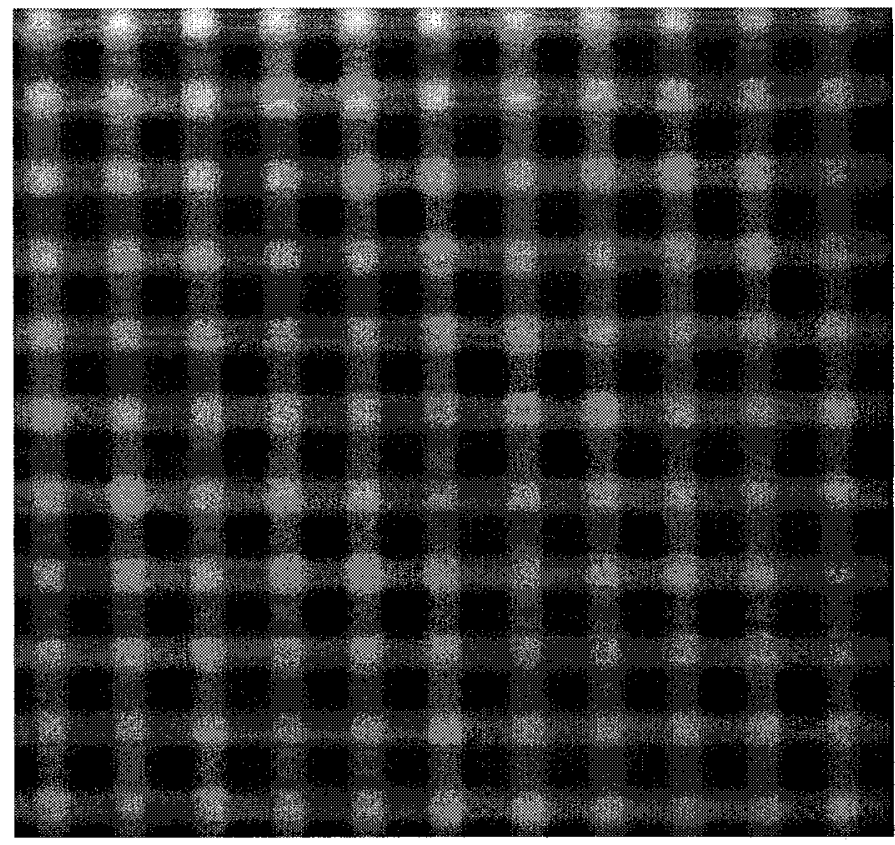

Figure 3.16 Composite image of an MLC configuration taken at collimator angle $0^{\circ}$ and $90^{\circ}$

Note that all images were taken without any gantry motion or movement of the imager system, ensuring a common beam isocentre for both images in the composite of Figure 3.15 and all images of figure 3.16 .

It is worthwhile to note that the MLC is machined with extremely high precision and accuracy. The grid of centroids $\left\{x_{f}(i), y_{f}(j)\right\}$ obtained through local thresholding are then projections of the intersection points of the middle of the even numbered MLC leaves from the two collimator rotations $0^{\circ}$ and $90^{\circ}$. Within the tolerance of the MLC manufacturing (better than $0.01 \mathrm{~mm}$ ), these intersecting points $\left\{x_{i}, y_{j}\right\}$ in physical space can be modeled knowing the leaf width $w$, the leaf index $i$ or $j$ and the location of the beam isocentre $\left(x_{i f}, y_{i f}\right)$, such that

$$
x(i)=\left\{\begin{array}{lc}
x_{\text {if }}+w(i-15.5) & i=16,18,20,22 \\
x_{\text {if }}+w(i-14.5) & i=8,10,12,14
\end{array} .\right.
$$

and 


$$
y(j)=\left\{\begin{array}{lc}
y_{i f}+w(j-15.5) & j=16,18,20,22 \\
y_{i f}+w(j-14.5) & j=8,10,12,14
\end{array} .\right.
$$

For a field of view of $16 \mathrm{~cm} \times 16 \mathrm{~cm}$, the geometric distortion is defined as the mean positional difference between the imaginary and physical spaces. We choose this size of field of view because it can cover most radiation portals in prostate cancer radiotherapy. We define the mean geometric distortion $\bar{\Theta}$ for each image as the quadratic average of the radius difference for all grid points, which can be calculated as follows:

$$
\bar{\Theta}=\frac{1}{N_{g}} \sum_{i, j} \sqrt{\left[x(i)-x_{f}(i)\right]^{2}+\left[y(j)-y_{f}(j)\right]^{2}},
$$

where $N_{g}$ is the total number of grids.

The deviation of the actual image $\left\{x_{f}(i), y_{f}(j)\right\}$ from the theoretical/physical grid $\{x(i), y(j)\}$ defines the geometric distortion in terms of the geometric transformation $T_{f p}$ required to map $\left\{x_{f}(i), y_{f}(j)\right\}$ back to $\{x(i), y(j)\}$. An affine transformation was applied and mapping matrix $T_{f p}$ was extracted using the least square fit to the following equation:

$$
\left(\begin{array}{l}
x \\
y
\end{array}\right)=T_{f p}\left(\begin{array}{l}
x_{f} \\
y_{f}
\end{array}\right)
$$

where $T_{f p}$ represents a linear mapping requiring scaling, rotation and skewing. In this case translation was removed since both $\left\{x_{f}(i), y_{f}(j)\right\}$ and $\{x(i), y(j)\}$ are relative to the same isocentre. 


\subsection{EPID Mechanical Instability}

Ideally, the orientation of the MLC leaves at collimator 0 is always parallel to the horizontal axis of a portal image while the collimator central axis always intersects a fixed pixel in the image. However, because of the poor mechanical mounting of the imager as well as gantry sag there is a significant rotation and translation of the imager at different gantry angles. In this study, we selected the apparent variation of MLC leaf orientation and beam isocentre as quantifiers of the mechanical stability of the electronic imager.

A clinical image acquired with the Beamview T1 can be divided into the open radiation portal, the area shielded by the MLC leaves and the area shield by the solid jaws. Two segments (the $X 1$ and $X 2$ banks), about $5 \mathrm{~cm} \mathrm{x} 10 \mathrm{~cm}$, in each of the regions containing interleaf leakage are selected from the image. Each region is at least $0.5 \mathrm{~cm}$ from the open field and image boundary. Analysis of this part of an image allows us to accurately measure differences between MLC leaf orientation in an image and the known orientation of the collimator (collimator rotation). The $\mathrm{Y}$ component of the position of the beam isocentre can be accurately determined by using Radon transform analysis of the interleaf radiation leakage, as described in detail in section 3.3.3.

\subsection{Results}

\subsubsection{Imager Rotation Test}

The ability of the Radon transform method to determine projection (collimator) angle with respect to the EPID grid was tested for five collimator angles $\left(0,1,2,3\right.$, and $\left.4^{\circ}\right)$. The angle between the collimator rotation and the EPID grid was determined independently using the projection of a machined grid and was known to better than 0.1 degree for each of the test angles. For each test case the inter-leaf radiation contrast signal was calculated as a function of projection angle $\theta$ in the same fashion as the example shown above in Figure 3.12. The accuracy of the method was tested using 3 
different sets of information: the inter-leaf leakage through a single leaf bank from a single image frame; the inter-leaf leakage through both leaf banks from a single image frame; and finally using the inter-leaf leakage behind both leaf banks from a composite image made from the sum of six successive frames. The results are shown in table 3.1. It can be seen that in all cases the radon transform method was able to determine the collimator angle with respect to image grid to within $0.4^{\circ}$. (Note that the manufacturers specification for all accuracy of all rotations, including collimator is $\pm 0.5^{\circ}$ ). Overall we are able to determine the collimator rotation to within $0.12 \pm 0.18,0.12 \pm 0.11$ and $0.08 \pm 0.11$ degrees by analyzing the inter-leaf radiation leakage under a single bank for 1 frame, both banks for 1 frame and both banks for six frames. The more inter-leaf radiation leakage information available, the more accurate the determination.

Table 3.1 Comparison of different measures for inter-leaf leakage analysis

\begin{tabular}{|c|c|c|c||}
\hline \multirow{2}{*}{$\theta_{c o l}($ Degrees $)$} & \multicolumn{3}{|c|}{$\theta_{\text {leaf }}($ Degrees $)$} \\
\cline { 2 - 4 } & X1 bank & Double bank & Frame Averaging \\
\hline 0.0 & 0.2 & 0.0 & 0.2 \\
\hline 1.0 & 1.0 & 1.2 & 1.0 \\
\hline 2.0 & 2.0 & 2.2 & 2.2 \\
\hline 3.0 & 3.0 & 3.2 & 3.0 \\
\hline 4.0 & 4.4 & 4.0 & 4.0 \\
\hline
\end{tabular}

\subsubsection{Isocentre determination test}

The ability of our method to determine the exact position of the intersection of the collimator rotation axis with the image plane was tested for 22 different MLC shaped fields. The location of $y_{i f}$ were determined directly from the Radon transform as described above. Similarly the $x_{i f}$ was determined by local thresholding and minimizing residuals from the planned positions of each leaf end. The "true" isocentre position was determined from the projection of a $0.5 \mathrm{~mm}$ steel sphere in a plastic plate placed in the 
collimator tray. The collimator was rotated and the isocentre was determined as the centre of the locus of projection images of the $0.5 \mathrm{~mm}$ sphere. For purposes of comparison the isocentre position was also calculated by the method of moments ${ }^{[52,55]}$ and the normalized cross correlation. ${ }^{[126]}$.

The results for the determination of isocentre tests are given in table 3.2, showing mean and standard deviation of the difference from the true isocentre for each of the three methods. Our results indicate that the Radon transform method presented herein provides a better estimate of the $\mathrm{Y}$ component of the isocentre (the direction perpendicular to leaf travel), while in the $\mathrm{X}$ direction (the direction of leaf travel) the method of moments provides the best estimate.

Table 3.2 Deviations from true isocentre for three methods

\begin{tabular}{|c|c|c|}
\hline \multirow{2}{*}{ Methods } & \multicolumn{2}{|c|}{ Deviations in mm } \\
\cline { 2 - 3 } & \multicolumn{2}{|c|}{$\mathrm{X}$} \\
\hline Leakage & $0.77 \pm 0.31$ & $0.63 \pm 0.25$ \\
\hline Correlation & $0.97 \pm 0.36$ & $0.85 \pm 0.37$ \\
\hline Moment & $0.72 \pm 0.36$ & $1.07 \pm 0.65$ \\
\hline
\end{tabular}

\subsubsection{Geometric Distortion Measurements}

For a $16 \mathrm{~cm} \times 16 \mathrm{~cm}$ checker board, the geometric distortion was measured in images acquired at four gantry angles, $45^{\circ}, 135^{\circ}, 225^{\circ}$ and $315^{\circ}$. Table 3.3 summarizes the means and range of the geometric distortions of the Beamview TI image chain. No significant difference in the geometric distortion was observed at the different gantry angles. The mean distortion was within $1 \mathrm{~mm}$. The geometric distortion is acceptable for patient setup and treatment field verifications because both MLC positioning uncertainty $^{[127]}$ and setup uncertainty ${ }^{[68]}$ are twice the distortion magnitude. It was also 
found that the distortion spatially varies over the examined area. The distortion was largest near the image corners, and was skewed along oblique directions in the image domain. Applying the measured transform matrix $T_{i p}$ to the original images, the mean geometric distortion can be reduced to less than $0.25 \mathrm{~mm}$.

Table 3.3 Geometric distortion means and ranges $(\mathrm{mm})$ for different gantry angles

\begin{tabular}{||c|c|c|c|c|}
\hline Distortion & Gantry $45^{\circ}$ & Gantry $135^{\circ}$ & Gantry $225^{\circ}$ & Gantry $315^{\circ}$ \\
\hline Raw Image & $0.75(0 \sim 1.66)$ & $0.74(0 \sim 1.70)$ & $0.72(0 \sim 1.66)$ & $0.72(0 \sim 1.72)$ \\
\hline Corrected & $0.25(0 \sim 0.75)$ & $0.25(0 \sim 0.75)$ & $0.25(0 \sim 0.75)$ & $0.25(0 \sim 0.75)$ \\
\hline
\end{tabular}

The correction method for geometric distortion was tested using images of a pelvic phantom with fiducial markers $(1.1 \mathrm{~mm}$ in diameter and $2 \mathrm{~mm}$ in length) Two $18 \mathrm{MV}$ images were taken 180 degrees apart.. The phantom was setup with the Centre of the Mass (COM) of the three fiducial markers placed at the machine isocentre and images were taken for gantry angles of $45^{\circ}$ and $225^{\circ}$. The two portal images should be identical when flipped horizontally. One of the opposed images was flipped and then the two images were fused. Figure 3.17 (a) is the super position of the two images prior to the distortion correction and Figure3.17 (b) is the fusion of the same two images after the distortion correction transform has been applied to each image. It can be seen in Fig 3.17 (a) that the fiducial markers are mismatched. In this case the composite image has them displaced by up to $2.5 \mathrm{~mm}$. After applying the mapping matrix, the mismatch of the fiducial markers is effectively eliminated as seen in Figure 3.17(b). The difference in magnification between the two opposed images was ignored because the distance between the markers and machine isocentre is less than $2.5 \mathrm{~cm}$, and thus the magnification difference does not result in significant apparent seed separation.

Although the magnitude of the geometric distortion in the portal image is small $(<1 \mathrm{~mm})$, the correction is still required in order to measure intra-fraction organ motion. In this work we have defined the intra-fraction organ motion to be the positional difference in 
fiducial markers between two opposed field images taken in the same fraction. The magnitude of the intra-fraction organ motion is anticipated to be comparable to the distortion ${ }^{[44]}$. Failure to remove distortion would make it very difficult to identify the source of the seed mismatched seen in Figure 3.17(a).

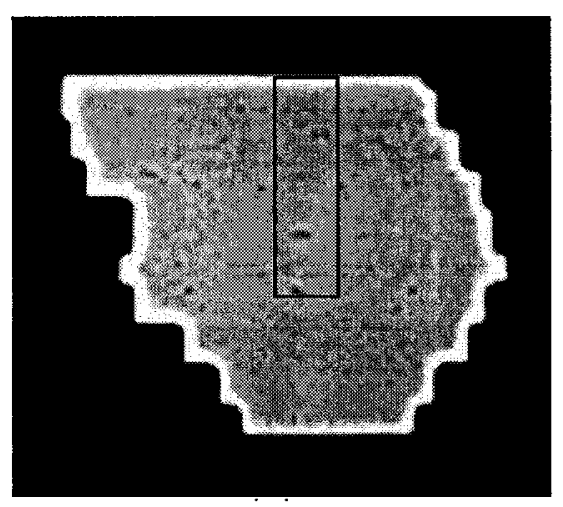

(a) Without correction

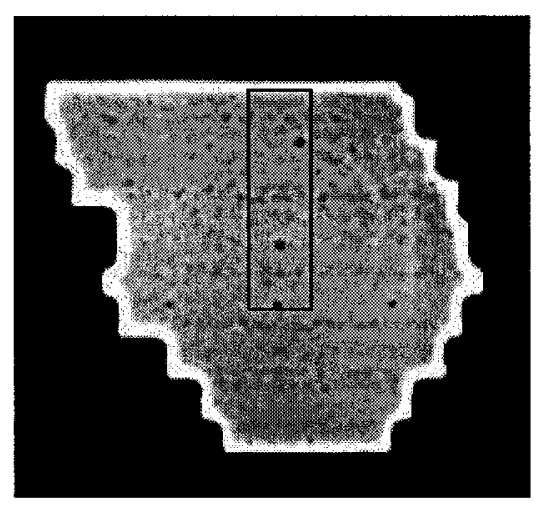

(b) After correction

Figure 3.17 Superposition of two opposed portal images without (a) and with (b) correction for spatial distortion

\subsubsection{EPID Mechanical Stability Measurements}

The mechanical stability of the Siemen's imager at different gantry angles, was measured using a calibrated isocentre jig consisting of a $0.5 \mathrm{~mm}$ sphere placed on the collimator axis of rotation. With the jig in place an $18 \mathrm{MV}$ portal image was taken at 8 different gantry angles. This was repeated three times over a period of one month. Figure 3.18 is a plot of the $\mathrm{X}$ and $\mathrm{Y}$ components of the isocentre as a function of gantry angle for one measurement. The variation of the isocentre in the MLC direction of travel (X component) was found to be $11.5 \mathrm{~mm}$ over a full range of gantry rotation while isocentre in the solid jaw moving direction (Y component) was measured to be less than $2 \mathrm{~mm}$. The data suggest a significant variation exists in the isocentre in the $\mathrm{X}$ direction. Several factors could cause this variation. The imager might be poorly installed onto the treatment machine, so the location of the imager with respect to the gantry head may vary 
with gantry rotation. Additionally, gantry sag during gantry rotation may also contribute to this variation.

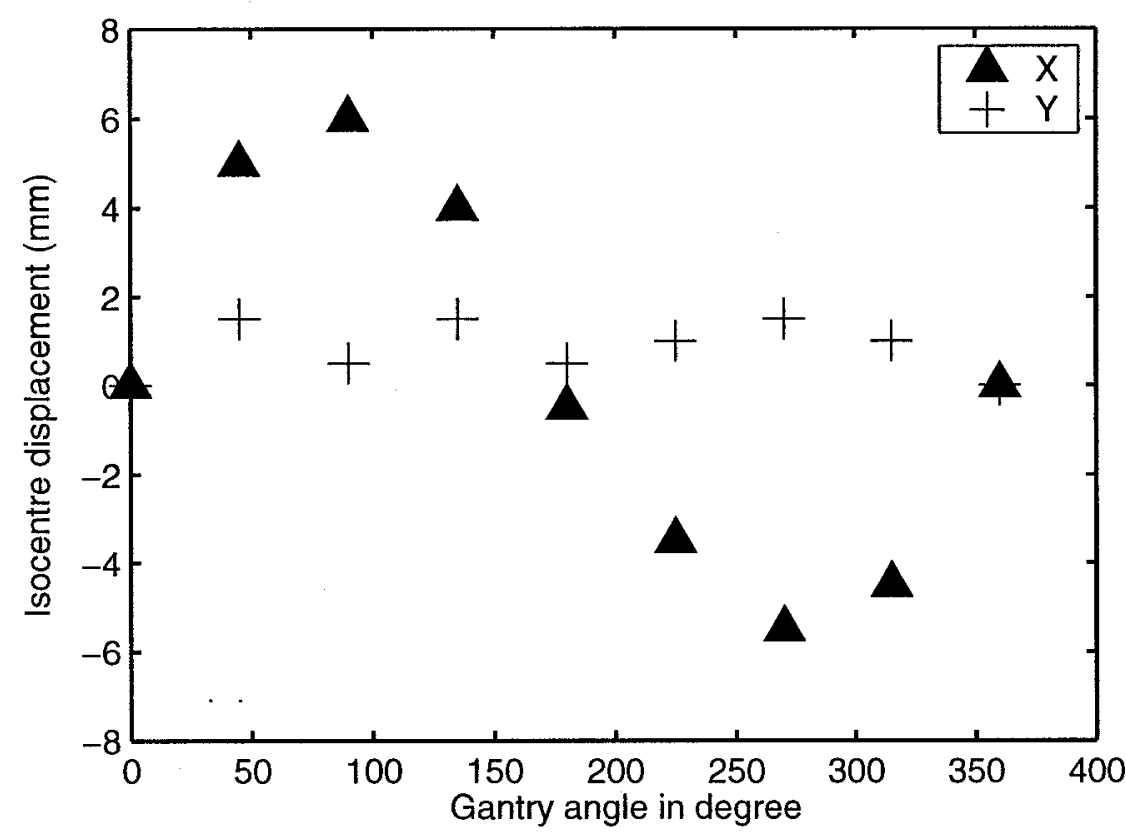

Figure 3.18 the isocentre displacement as a function of gantry angle (gantry 0 was chosen as a reference)

We have also determined the beam isocentre in 257 clinical images using the interleaf radiation leakage information at four gantry angles $45^{\circ}, 135^{\circ}, 225^{\circ}$ and $315^{\circ}$ over a period of 9 months. The standard deviations of the measured isocentre position are shown in Table 3.4. The isocentre variation in the MLC traveling direction $(0.19 \sim 0.25 \mathrm{~mm})$ was found to be smaller than in the solid jaw moving direction $(1.89 \sim 1.93 \mathrm{~mm})$. This can be explained as follows. The solid jaw moving direction is also the direction of the longitudinal extension of the imager when a portal image is taken. There is considerable variation in the imager position in this direction. There is no indicator to show whether or not the imager is fully extended and the locking system is not solid. Furthermore, frequent operation (pull out and fold back) of the imager may cause a systematic drift in the imager extension direction. 
In Figure 3.19, the $\mathrm{Y}$ component of the beam isocentre is plotted as a function of time during our observation period from Sept. of 2002 to June of 2003. The result shows that the $\mathrm{Y}$ component isocentre did shift $6 \mathrm{~mm}$ towards the extension direction of the EPID during the observation period.

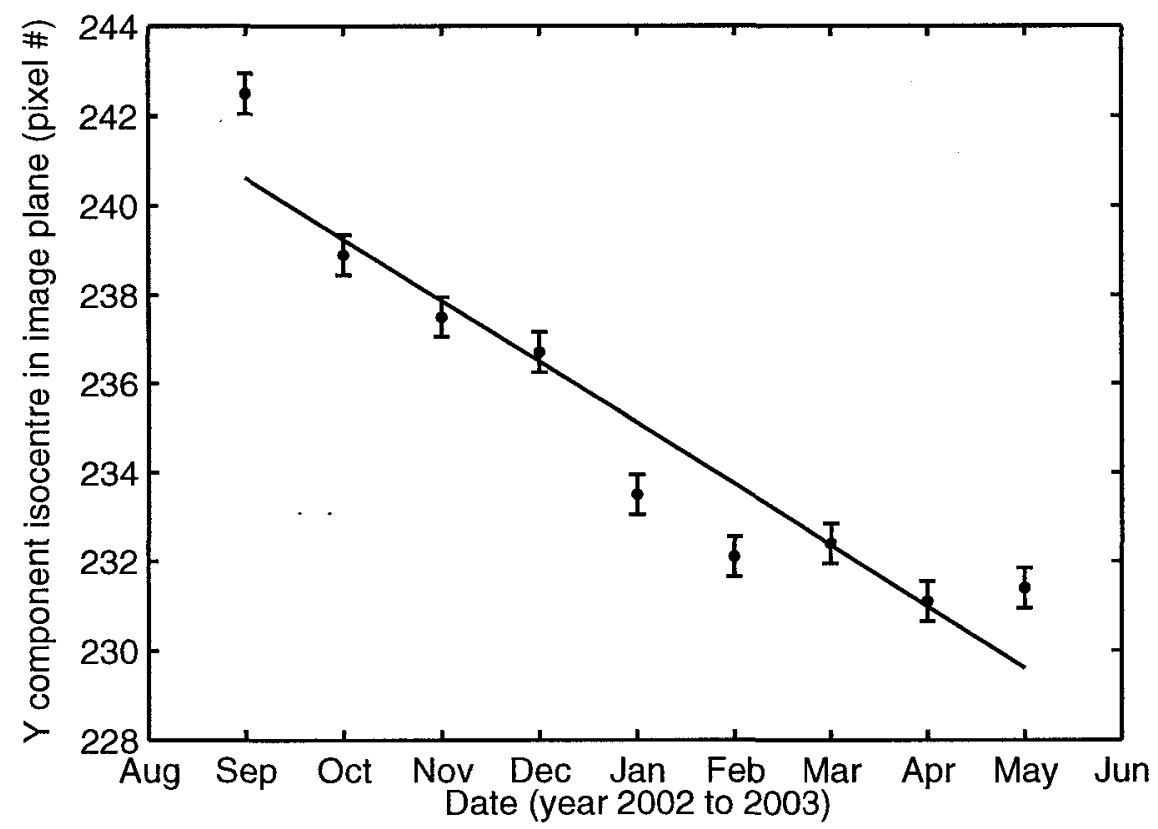

Figure 3.19 Isocentre drift as a function of time

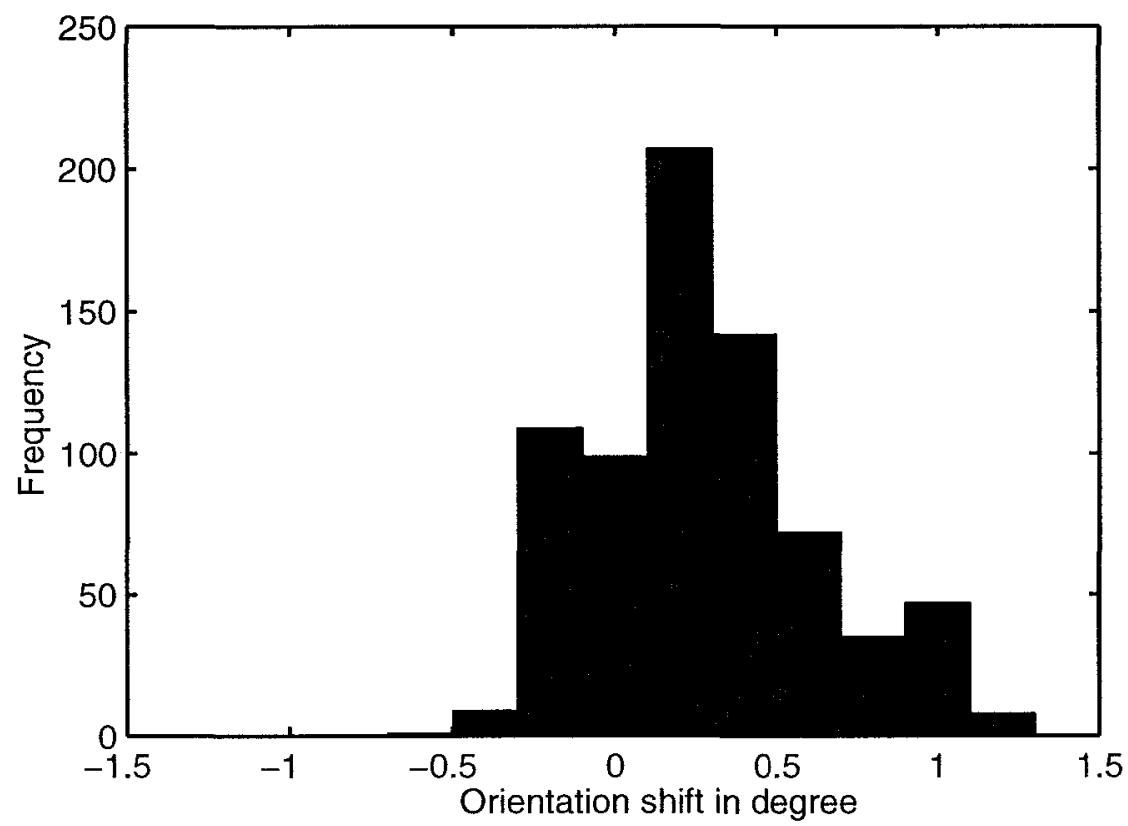

Figure 3.20 Histogram of imager orientation shift 
The orientation of the imager was determined with the algorithm of inter-leaf radiation leakage for 729 clinical images over a period of 9 months. The algorithm for the orientation of MLC leaves is described in section 3.3.3 Figure 3.20 plots the frequency distribution of the orientation over 729 measurements. We found that the standard deviation of the orientation distribution of the imager is $0.35^{\circ}$. This uncertainty could result from either the uncertainty in the collimator rotation or the mechanic instability of the imager itself.

Table 3.4 Distribution of beam isocentre for different gantry angles

\begin{tabular}{||c|c|c|c|c|}
\hline Direction & Gantry 45 & Gantry 135 & Gantry 225 & Gantry 315 \\
\hline X component & $0.25 \mathrm{~mm}$ & $0.18 \mathrm{~mm}$ & $0.23 \mathrm{~mm}$ & $0.21 \mathrm{~mm}$ \\
\hline Y component & $1.901 \mathrm{~mm}$ & $1.89 \mathrm{~mm}$ & $1.93 \mathrm{~mm}$ & $1.92 \mathrm{~mm}$ \\
\hline
\end{tabular}

\subsection{Discussion \& Conclusions}

\subsubsection{General Discussion}

In our experience, the mechanical instability of the Siemens TI portal imager has acted as a barrier to fully exploiting portal images for linear accelerator quality assurance and for accurately distinguishing between patient setup errors and MLC leaf/jaw calibration errors. In this work we have investigated the feasibility of using inter-leaf radiation leakage to accurately determine collimator rotation and the position of the beam isocentre in the EPID image. We have shown that the Radon transform can successfully extract the position and the orientation of inter-leaf leakage from the electronic portal image and that this information can be used to accurately determine the collimator rotation and the location at which the collimator axis of rotation (beam isocentre) intersects the EPID image plane. Electronic portal imagers could be ideal devices for filmless Quality Assurance of treatment machines, particularly MLC and collimator calibration, but are not being employed for this purpose in most radiotherapy practices. A recent report ${ }^{[117]}$ 
indicated that at the end of 2002, only 5\% of U.S. cancer centres employed electronic portal imagers for filmless Quality Assurance. Among the barriers to implementation are

poor image quality and mechanical instability. We believe that the methods presented herein can in part address many of these concerns.

The efficacy of portal imagers as a tool for image-guided radiotherapy is also limited by their performance, including their lack of mechanical stability/reproducibility (orientation and translation) from field to field and fraction to fraction as well as their low image contrast and inherent geometric distortion. We have demonstrated that our method can overcome the issue of mechanical instability and that we can accurately determine an exact reference position (e.g. the intersection of the collimator axis of rotation with the image plane). Armed with this information it is possible to assess clinical portal images to distinguish between geometric errors arising from patient setup as opposed to geometric misses arising from errors in MLC leaf position or collimator rotation. As discussed later in this thesis we use this approach to analyze EPID images from a large series of prostate patients to accurately quantify inter-fraction setup errors, intra-fraction patient motion, prostate motion and treatment specific daily errors in MLC calibration.

\subsubsection{The Effect of Distortion and Mechanical Stability on Beam Isocentre}

If the Beamview TI imager was rigidly mounted onto Siemens linear accelerator, the collimator central axis would always be projected onto a fixed pixel in a portal image over a full range of gantry rotation. Our measurements indicated that it is valid only for the $\mathrm{Y}$ component of the beam isocentre. The variability in the $\mathrm{X}$ component of the isocentre was found to be $11.5 \mathrm{~mm}$ among observed gantry positions. This implies that the position of imager detector relative to the treatment head is gantry position dependent. On the other hand, the $\mathrm{Y}$ component of beam isocentre drifted up to $5.5 \mathrm{~mm}$ during a period of 9 months. This suggests that the $\mathrm{Y}$ component of the isocentre in the portal image is temporally dependent. Overall, both components of isocentre are either spatial (treatment field) or temporally (fraction) dependant. The approach of using a look-up able for determining the isocentre in a portal image is therefore not useful. Because beam 
isocentre is the only reference to measure inter- and intra-fraction target motion, the measurement would be inaccurate without correcting for the mechanical instability of both the linear accelerator and the electronic imager. The uncertainty in field orientation was found to be $0.27^{\circ} \pm 0.35^{\circ}$, less than the collimator tolerance of $0.5^{\circ}$. Its impact on the beam isocentre can be ignored. The geometric distortion unlikely affects the accuracy of isocentre determination because it is radius dependent and there is less distortion presented in image centre where the isocentre is located.

\subsubsection{The Effect of Distortion on Marker Positioning}

Our measurements demonstrated that the distortion in the image plane is location dependant. It can affect the accuracy of the feature locations (seed or bony marker) if the feature is located in the most distorted image area (e.g. corners). The spatial distortion problem confused us for a long time in the determination of the intra-fraction prostate motion by using two opposed field images. This is discussed as follows.

Our prostate phantom experiments demonstrated a clear seed mismatch following accurate image fusion. However, there was no phantom motion involved between two image acquisitions. This must be due to something wrong with the imaging system. Firstly, we suspected that seed mismatch could have happened if the gantry angle difference between two fields is not exactly 180 degree. We verified the gantry rotation with the digital smart level for three weeks without finding any gantry rotation related problem. Secondly, we suspected that there might be a systematic MLC positioning error. A systematic error of $1.5 \mathrm{~mm}$ in $\mathrm{X} 1$ bank would produce similar errors, but this was excluded following the MLC positioning calibration. Subsequently we verified that the accuracy of the image registration between two opposed field images. For each of two gantry angles, a calibrated jig was placed at the path of beam isocentre, two phantom images were then obtained, and it was found that the difference in actual isocentre and algorithm-based isocentre is less than $1 \mathrm{~mm}$ for all test cases. The uncertainty in image fusion cannot lead to a mismatch of $2.5 \mathrm{~mm}$ either. Eventually it wa determined that 
geometric distortion was the source of the seed mismatch. The obtained correction matrix as described in section 3.4 removes the spatial distortion.

\subsubsection{Clinical Applications}

Methods used to correct existing geometric distortion in an image have been studied by several investigators. ${ }^{[119,128]}$ The methods themselves are straightforward. The acquiring of data for the determination of the appropriate correction methods is not always trivial. It often involves a large amount of work to measure grids of a specific phantom in physical space and their projection in the portal image. The creation of a well-defined geometric grid in this work avoids the complicated phantom measurement and makes it relatively easy to perform. This is the main difference between our method and others. The advantage of this approach is that the width of a MLC leaf is chosen as reference, the position of each leaf relative to the collimator axis is fixed and well defined. In contrast, any phantom measurements for determining geometric distortion require a beam isocentre as reference, but it is not easy to obtain in conventional phantom experiments because edge information and feature information are unavailable. If a jig is utilized, it must be calibrated before the phantom experiment. In addition phantom measurements need a magnification correction. MLC leaves do not have magnification issue, because the width of each leaf is always projected to be $10 \mathrm{~mm}$ at isoplane. The accuracy of this correction for geometric distortion only depends on the manufacturer performance of the MLC. 


\section{CHAPTER 4 \\ A Study of Prostate Delineation Referenced Against a Gold Standard Created from the Visible Human}

\subsection{Overview}

This chapter summarizes our study of inter- and intra-observer error in the delineation of the prostate organ, performed at The Ottawa Hospital Regional Cancer Centre (TOHRCC). Section 4.1 discusses the role of target delineation in the context of the entire treatment process and section 4.2 describes the methods by which the reference gold standard or "true" prostate volume was established based on the Visible Human Project (VHP) data. It also describes the methods of determining inter- and intraobserver difference relative to the gold standard. Sections 4.3 presents the results of the work and Section 4.4 discusses the clinical implications of the results.

\subsection{Introduction}

It is generally accepted that the individual sub-processes that constitute external beam radiation therapy can affect patient outcome, and specifically that geometric uncertainties play a major role in tumour control and normal tissue complications. ${ }^{[25,129]}$ Historically, the lack of three-dimensional imaging, both for treatment preparation and planning, and during delivery has resulted in the spatial accuracy of radiation therapy delivery equipment being significantly better than the accuracy/resolution with which the threedimensional target volume could be determined within the patient. For many years the radiation therapy physics community has devoted a significant proportion of its overall research effort to a reduction in dosimetric uncertainties rather than patient related geometric uncertainties. ${ }^{[7]}$ There has also been significant effort directed to improving the geometric accuracy of radiation therapy simulation and delivery equipment through 
enhanced quality control procedures. ${ }^{[75,94,109,130]}$ Recent technological developments in imaging and radiation therapy delivery, such as CT, MR, and linear accelerators with on board imaging have made it possible to measure, categorize, document and understand patient based geometric uncertainties. ${ }^{[3,7,79]}$ Today, tumour target delineation along with the daily patient positioning error and internal target motion are recognized as three major sources of error in the overall radiation therapy process. ${ }^{[68,116]}$ Although identified as a critical component in the cancer treatment process, the accuracy and quality of target delineation has yet to be addressed by either AAPM or ICRU reports.

The accuracy with which the CTV can be defined on images acquired by various modalities is not well documented for most tumour sites ${ }^{[25]}$. The ability of a physician to delineate targets will likely vary with imaging modality, cancer site, disease stage, as well as patient age and size. The physician's determination of the CTV. will also depend, by definition, on the experience and knowledge of the individual physician. In early stage, low risk prostate cancer the entire organ (prostate) is considered the target (CTV) of radiation therapy although there are presently some clinical investigations looking at sub organ definition of the GTV using functional CT, MR and PET images. The prostate and much of its surrounding normal tissues have similar mass attenuation coefficients, making accurate target definition on CT, that is segmentation of the prostate from adjacent tissues, a difficult task for the radiation oncologist. It is fairly well documented that there is variation in tumour definition between and amongst different observer groups (e.g. oncologists, anatomists, urologists and therapists) and this has been investigated by several researchers for different disease sites. ${ }^{[26,28,33,131-133]}$ Importantly, to our knowledge, none of these studies have reported their findings against a true gold standard. Most have used a population average as the reference value and measured variation against this average, making it impossible to extract systematic error from the data.

The work described in this chapter uses data from the Visible Human Project ${ }^{\circledR}$ which provides a complete set of cross-indexed MR, CT and anatomical photographic digital images of an entire human male cadaver. Our study uses the anatomical images to 
develop a gold standard for the exact extent of the prostate and takes advantage of data set indexing to allow comparison of CT based organ delineation with the gold standard derived from the axial anatomical photographic images. The anatomical (photo) images provide much finer detail than the CT images and hence serve as a better means to estimate the boundaries of the true organ. The anatomical images are 32 bit color, with a pixel resolution of $0.33 \mathrm{~mm}$. In comparison the CT images are 8 bit grey scale with $0.8984 \mathrm{~mm}$ pixel size. An expert group consisting of an urologist, a radiation oncologist and an anatomist, created the gold standard volume from the photo images. Six radiation oncologists then defined the prostate alone on the corresponding CT images allowing us to measure and quantify inter and intra observer random and systematic (with respect to the gold standard) errors.

\subsection{Materials and Methods}

The CT and anatomical images of the visible human male form the basic data set for this study. Before being useful in this study, both sets of images required preprocessing. The CT images were imported into our radiation treatment planning system for target (prostate) delineation by the radiation oncologists while the anatomical images, used as the basis for the gold standard, were imported into Photoshop 7.0 (Adobe, CA, USA) for segmentation. The CT and anatomical image data sets were co-registered to allow comparison of volumes defined on each set of images. Finally the data sets were compared and analyzed for random and systematic error.

\subsubsection{The Visible Human-Male Data Set}

This dataset consists of MR, CT and anatomical images, the later being acquired simultaneously with the sectioning of the cadaver. The CT data consists of 8 bit grey scale axial slices, $3 \mathrm{~mm}$ thick, each pixel being $0.8984 \mathrm{~mm} \times 0.8984 \mathrm{~mm}$. The anatomical photo set is comprised of 32 bit deep color images, with a slice interval of 1 $\mathrm{mm}$ and a pixel resolution of $0.33 \mathrm{~mm} \times 0.33 \mathrm{~mm}$. The complete male data set, 15 gigabytes in size, was made available in November 1994. The TOHRCC obtained 
permission to use these data for target delineation from US National Library of Medicine. Figure 4.1 (a) is an example of an anatomical image cross section through the pelvis and figure 4.1 (b) is the corresponding CT image.

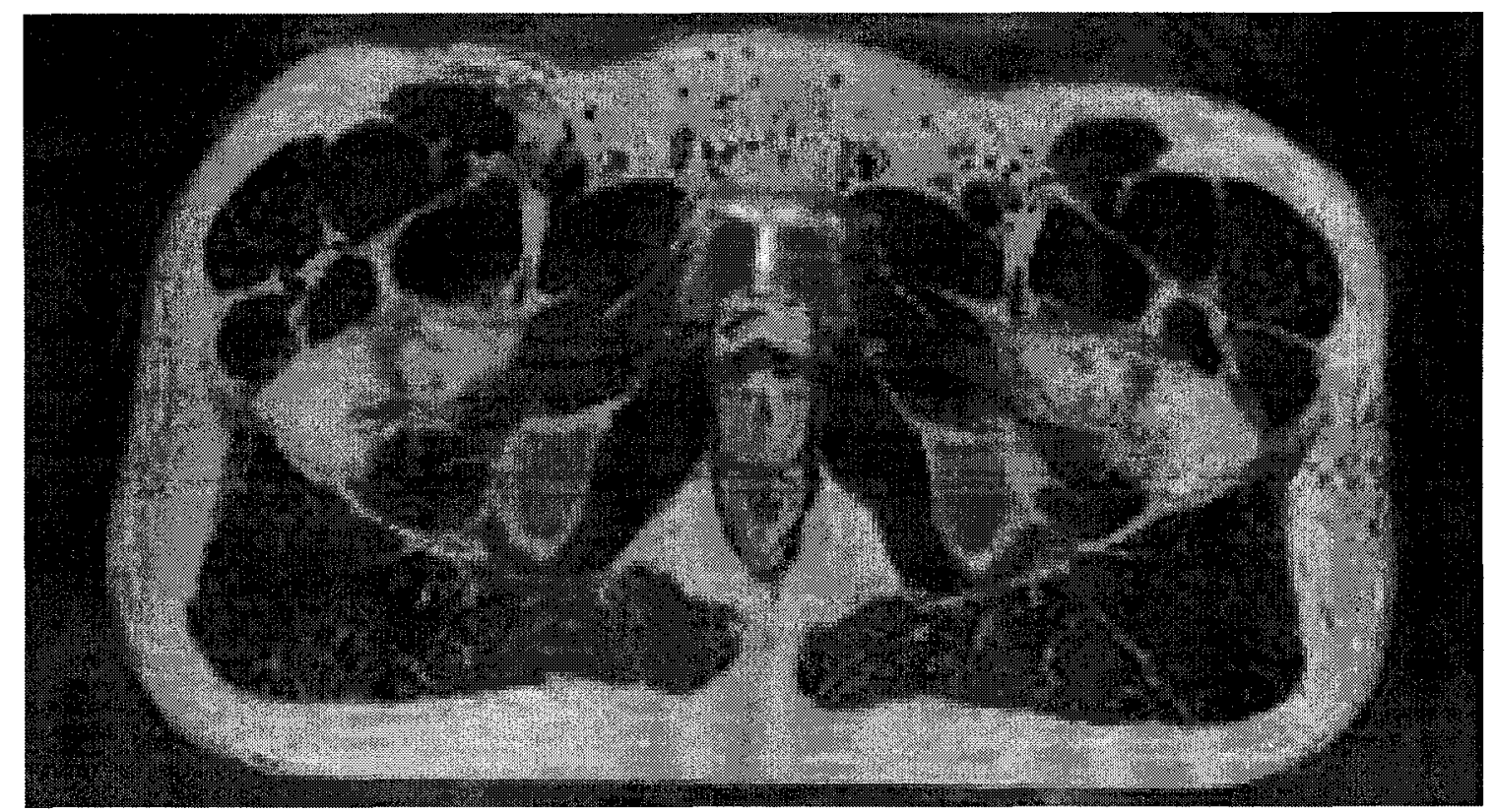

(a) Anatomical image of pelvic region

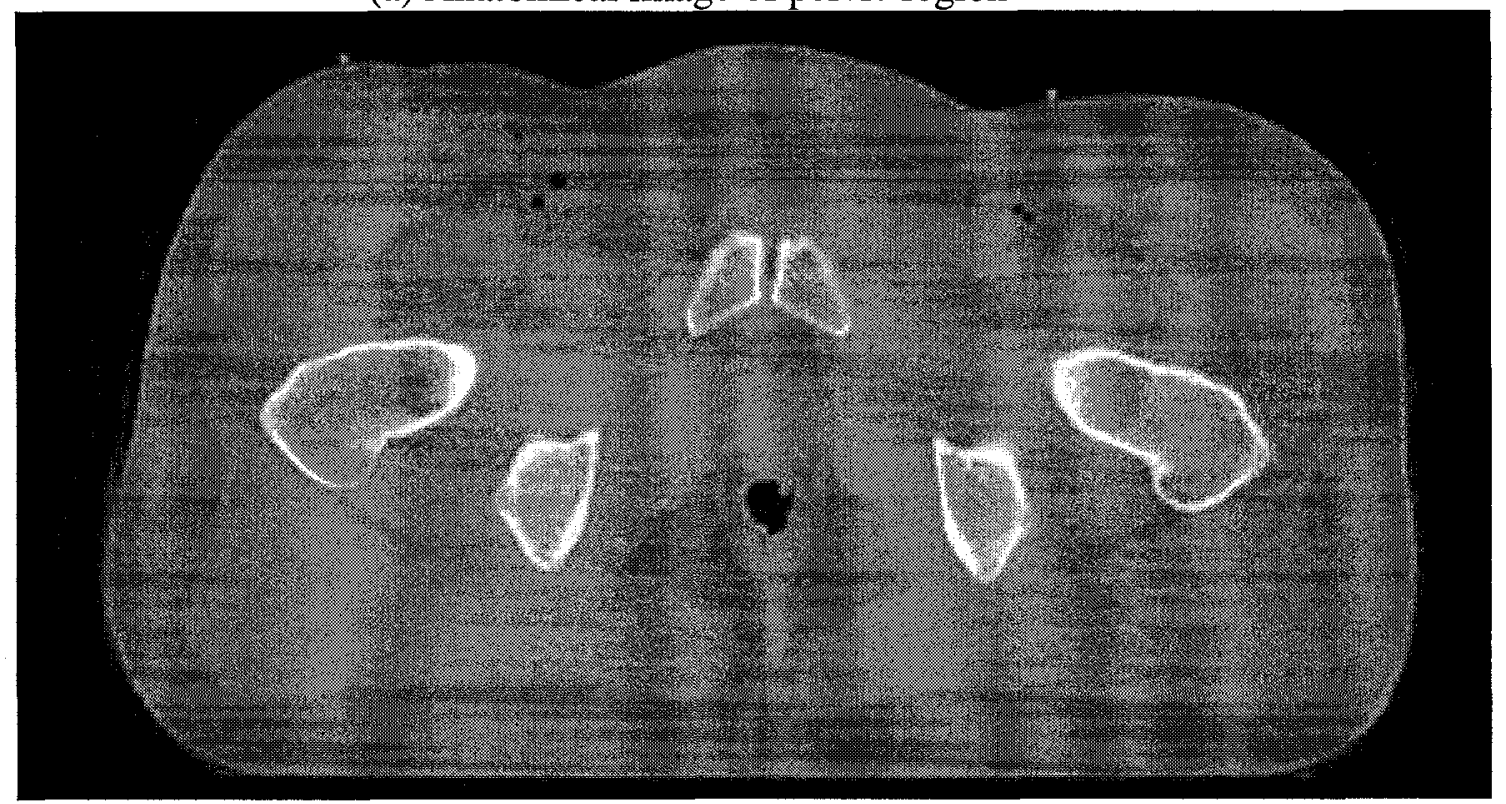

(b) Corresponding CT image

Figure 4.1 (a) an axial photo image of the pelvic region and (b) corresponding CT 


\subsubsection{Observer and Gold Standard Groups}

Six board certified radiation oncologists at The Ottawa Hospital Regional Cancer Centre participated in the CT based target delineation portion of this study. Five were senior radiation oncologists, each with more than 10 years work experience in CT based target definition and treatment planning for prostate cancer, while the sixth was a recent royal college graduate. A multi-disciplinary group of experts, working in concert, were asked to define the "true" prostate on the anatomical photo images. This group consisted of an urologist, a radiation oncologist and an anatomist.

\subsubsection{Determination of The Prostate Gold Standard Volume}

The "true" prostate as defined by the expert group on the anatomical photo images, was. established as the gold standard for this study. The graphics software package Photoshop $^{\circledR}$ 7.0(Adobe Inc, CA) was used as the platform for photo image analysis because it reads images in raw format and provides tools to create, edit and store contour points. Prior to the exercise of delineating the true prostate, the expert group was trained in the use of Photoshop. On each anatomical slice containing a cross section through the prostate, the exact boundary of the prostate was manually segmented. The contour data on each slice was accepted only when consensus was reached amongst the group. In a similar fashion the experts were also asked to define the true rectum.

The three dimensional volume of the prostate (or rectum) was constructed from the ensemble of contours from all slices, each contour consisting of a set of discrete points. The surfaces of the reconstructed prostate and rectum are shown in Figure 4.2. The gold standard for the "true" prostate is then accepted as the summation of the voxels as defined above, with each voxel having a unit volume $1.089 \times 10^{-4} \mathrm{~cm}^{3}$. Due to the spatial resolution in the axial direction $(1 \mathrm{~mm})$, the surfaces of the reconstructed volumes are not smooth, but display a staircase effect. For display purposes only, the reconstructed volumes were smoothed by convolution with a box kernel and the result is shown in Figure 4.3. 


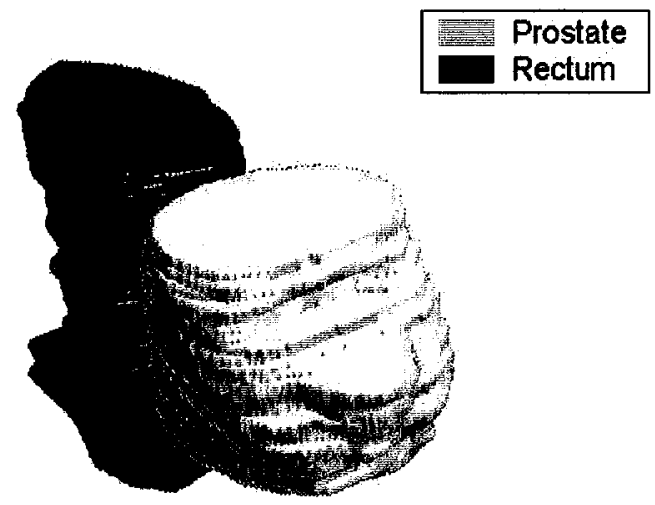

Figure 4.2 Rendering of the gold standard prostate and rectum

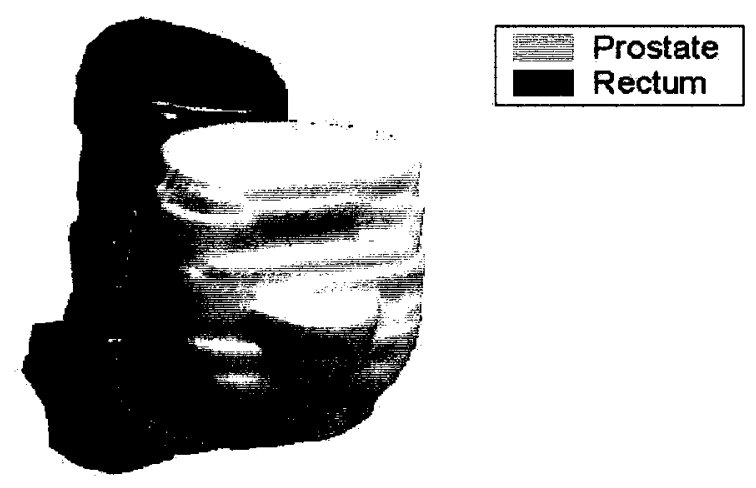

Figure 4.3 Rendering of the gold standard prostate and rectum after smoothing

\subsubsection{Delineation of the Prostate Volume on CT}

To be consistent with TOHRCC standard clinical practice it was felt necessary to use one of the standard clinical systems [TheraPlan ${ }^{\circledR}$ plus 3.0 (MDS Nordion, Canada) or 
ACQSIM $^{\circledR}$ (Philips Oncology system, USA)] as the platform for CT based target delineation. The VHP CT images were provided in GE SIGMA-5 raw format and were converted to DICOM for import using the DICOMatic ${ }^{\circledR}$ software package from TOMOVISION Inc. (Montreal, Canada).

Each observer was asked to contour the entire prostate a total of 20 times, each contouring session occurring a minimum of 3 days apart. During target contouring, the radiation oncologists were allowed to adjust window and level as well as contrast and brightness to their own liking.

Each Radiation Oncologist was asked to delineate the CTV as the prostate only (not to include the seminal vesicles) such that the prostate as contoured on CT could be compared directly to the gold standard defined by the expert group on the anatomical photos. Each of the 20 contours, from each of the six radiation oncologist, was extracted from the images for off line analysis using in-house software.

\subsubsection{Image Registration}

In the VHP data, anatomical cross-sectional images are $1 \mathrm{~mm}$ thick and are indexed (referenced) to the $3 \mathrm{~mm}$ thick axial CT images in the axial direction, but not in the two dimensional plane of each image. In order to register the images in this plane, we have chosen the external contour of the body as the reference because it is easily segmented without contrast enhancement and both the external contour and all internal structures and organs can be considered as solid, non-deformable objects since both sets of images were acquired from the frozen cadaver. Following segmentation of the external contours, rigid body registration is performed by a transform determined by centroid matching and a rotation obtained by normalized cross correlation. The centroid, calculated using the first order moment of the body contour, is rotationally invariant because the objects are non deformable. The rotation $\theta$ between the two contours is determined by the method of cross correlation. 
Let the centroid of the body contour on an axial CT be given as $\left(x_{c t}^{c}, y_{c t}^{c}\right)$ while the centroid of the body contour on the corresponding anatomical image is $\left(x_{p h}^{c}, y_{p h}^{c}\right)$, then a point $\left(x_{c t}, y_{c t}\right)$ in the $\mathrm{CT}$ image can be registered (mapped) to the corresponding photographic image using the transformation:

$$
\left(\begin{array}{l}
x_{p h} \\
y_{p h}
\end{array}\right)=M_{c t}\left(\begin{array}{cc}
\cos \theta & -\sin \theta \\
\sin \theta & \cos \theta
\end{array}\right)\left(\begin{array}{l}
x_{c t}-x_{c t}^{c} \\
y_{c t}-y_{c t}^{c}
\end{array}\right)+\left(\begin{array}{c}
x_{p h}^{c} \\
y_{p h}^{c}
\end{array}\right)
$$

where $M_{c t}$ is the ratio of the spatial resolution between two modalities. In the visible human case, we have determined $M_{c t}$ to be 2.69. Three CT - Photo image pairs were selected (photo slices \#1894, \#1906 and \#1912) representing transverse slices through the

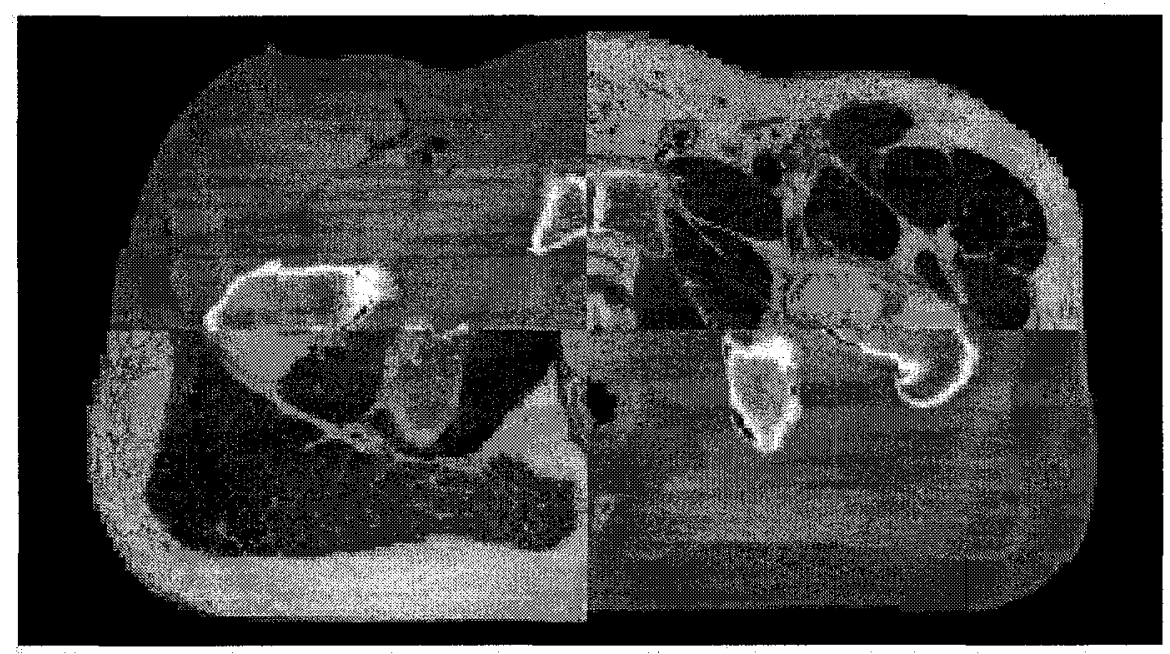

Figure 4.4 Checkerboard of the CT and corresponding photo images following 2D fusion

approximate superior, middle and inferior portions of the prostate. On each of the slice pairs, the external contours were extracted and matched as per the transform of equation 4.1. An average transform was then determined and used to fuse the full CT image set to the full anatomical image set over the region of interest. The correctness of this image 


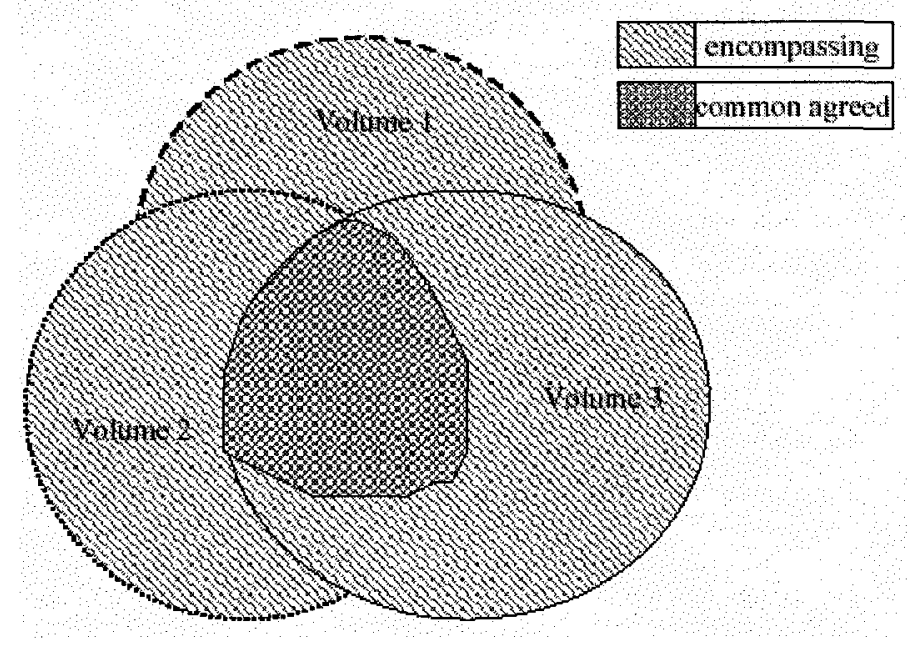

Figure 4.5 Definitions of the encompassing and common agreed volumes

fusion was estimated by comparing common and encompassing areas (see section 4.2.6) for the merged external contours on each slice. The mean distance between the common and encompassing volumes was found to be less than $0.15 \mathrm{~mm}$. Figure 4.4 is a checker board image showing the fusion of the CT and anatomical images for slice \# 1896.

\subsubsection{Geometric and Volume Analysis}

This work adopts the definitions of common agreed upon and encompassing volumes $\left(\operatorname{Rasch}^{[26]}\right.$ ) to quantify intra- and inter- observer variation in target volume definition. Simply, the common agreed upon volume is the intersection of all voxel sets and the encompassing volume is the union of all voxel sets. The relationship between the common agreed upon and the encompassing volumes is displayed in Figure 4.5. The Ratio of the Common agreed volume to the Encompassing volume (RCE) in a set of observations is then used as an indicator to describe the variability among multiple delineations by an individual observer or by a group of observers. The smaller the RCE value the larger the variability in a set of observations, while an RCE value of 1.0 indicates zero variation amongst observations in the set. 
We also define a function $f_{T I}$ that we refer to as the "goodness" of target definition. This is the percentage of "true" target included in an individual delineation and is given as:

$$
f_{T I}=\frac{V_{G S}^{C T}}{V_{G S}} \times 100 \%
$$

where $V_{G S}^{C T}$ is the common agreed volume between a CT delineated target and the "true" gold standard prostate volumes $V_{G S}$ defined by the expert group. The larger $f_{T I}$ the more "true" prostate is included in a specific volume delineation.

In addition to the volume difference we also measure the geometric error. This is done in several ways, one being gap analysis described below and the other being the vectoral distance from the geometric centre $V_{C T}(x, y, z)$ of a CT delineated prostate volume to the geometric centre $V_{G S}(x, y, z)$ of the "true" prostate volume. The geometric centre is determined as the centroid and is calculated as the first order moment of the surface contour.

\subsubsection{Gap Analysis}

An analysis of the prostate volume contoured on CT with respect to the gold standard can provide a global view of how well a delineated prostate agrees to its true volume. Clinically it may be more relevant to assess the distance to boundary agreement as a function of direction, since this can provide useful clinical information for determining PTV margins. Specifically we would like to determine the random and systematic errors in the principal directions; inferior/superior, anterior/posterior, left/right. It is also important to determine if radiation oncologists are more likely to include normal tissues or to miss tumour volume in their prostate target delineations and if these are directionally dependent. 
We have chosen the geometric centre of the true prostate to act as the origin on each slice and have defined a standard Cartesian axis (Ant/Post and Left/Right). Along each axis (positive and negative) we measure the distances between the CT based contour and the gold standard, which then defines the "gap" as depicted in Figure 4.6. Target is missed if the gap is negative (white in Figure 4.6) and positive if normal tissue is inappropriately included.

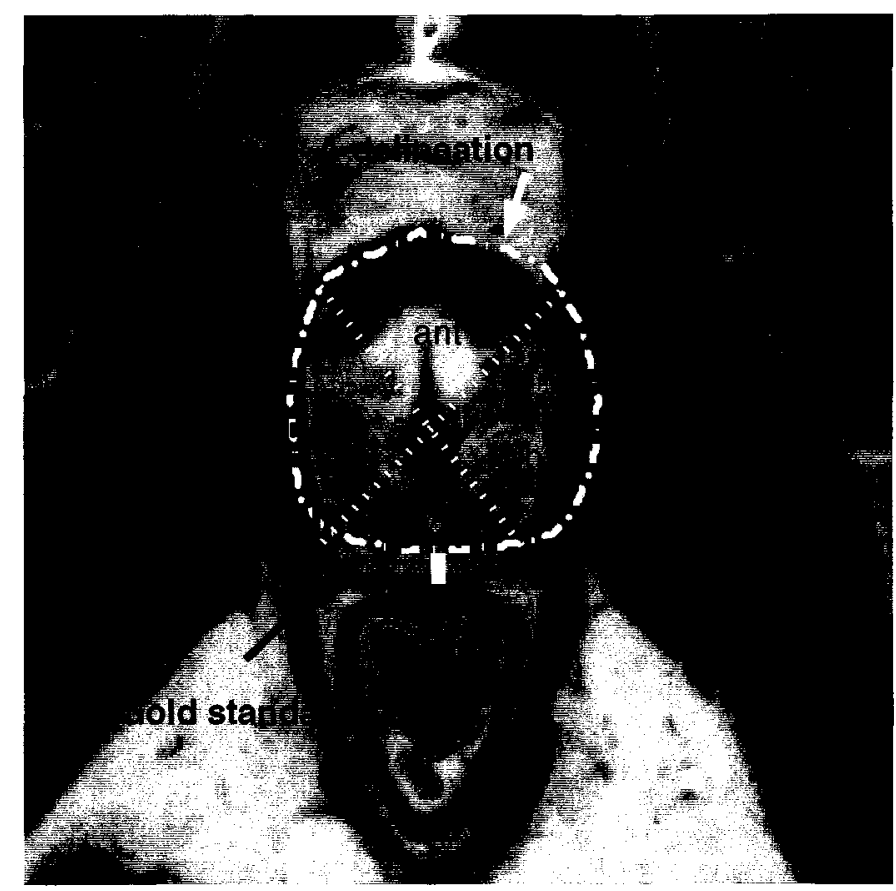

Figure 4.6 Gap representation between gold standard and corresponding delineated slices

\subsubsection{Quadrant based Probability Volume Histograms}

As depicted in Figure 4.6, the true prostate in each photo slice has been divided into four quadrants (left, right, anterior and posterior direction) about its geometric centre. The number of times that an individual voxel of the true prostate was included in an oncologist's delineation was then counted. Introduced here is a new concept, similar to the common integral Dose Volume Histogram (DVH), and named as a Probability Volume Histogram (PVH). The PVH is a cumulative or integral distribution of 
normalized frequency of inclusion. This concept has been applied to both target and normal tissue voxels.

\subsubsection{Statistical Analysis}

The statistical analysis of the volumes delineated by the various observers follows similar to the method described by van Herk ${ }^{[68]}$. Here we define:

$\sigma_{t d}(i)$ : The standard deviation in target delineation by a single observer $i$

$\overline{\Delta V}(i)$ : The systematic error for observer $i$, calculated as the mean difference between each volume delineation for that observer and "true" or gold standard prostate volume

$\mathrm{M}_{\mathrm{v}}$ : The overall systematic difference between the delineated prostate and the gold standard for all observations and calculated to be the mean volume difference between the 120 delineated volumes and the "true" volume

$\Sigma_{t d} \quad$ The inter-observer uncertainty in volume delineation, calculated as the standard deviation of the individual systematic errors $\overline{\Delta V}(i)$.

$\sigma_{t d}$ Overall random uncertainty, or group intra observer uncertainty, is defined as the quadratic sum (variance) of the individual observer uncertainties

Such that

$$
\begin{gathered}
\overline{\Delta V}(i)=\sum_{k=1}^{20}\left(V_{i, k}-V_{g s}\right) / 20 \\
\sigma_{t d}(i)=\operatorname{std}\left(V_{i, k}-V_{g s}\right) \\
M_{v}=\sum_{i=1}^{6} \Sigma_{i} / 6 \\
\Sigma_{t d}=\operatorname{std}(\overline{\Delta V}) \\
\sigma_{t d}=\sqrt{\sum_{i=1}^{6} \sigma_{t d}^{2}(i) / 6}
\end{gathered}
$$


where $V_{i, k}$ is the $\mathrm{k}^{\text {th }}$ observation by observer $i$ and $V_{g d}$ is the gold standard.

Similar definitions are used for the analysis of the geometric gap. (Note that equation 4.5 is only valid because each observer makes the same number of observations)

\subsection{Results}

A gold standard prostate volume for the visible human male was established by a group of experts based on anatomical photographic images. The prostate volume for the same subject was also contoured on co-registered CT images by six different observers, each observer repeating the target delineation a total 20 times. In total 120 prostate delineations were obtained. Subsequently the CT and anatomical images were fused and measures of agreement between the two sets were calculated as described above.

Table 4.1 shows the data for the six individual observers. As can be seen all observers delineated a volume that was greater than the true volume of $20.4 \mathrm{~cm}^{3}$. We have found that intra observer variation was small, the maximum standard deviation being less than $8 \%$ of the mean while the variation between observers was large, the mean volume changing by a factor of 1.5 from the largest to smallest volume.

The mean and standard deviation of the percentage of true prostate volume $\left(f_{T I}\right)$ included within the target defined by each observer ranged from a low of $79 \%$ to a maximum of $87 \%$. There was very little intra observer variation, ranging from $2 \sim 3 \%$ for all observers.

It is interesting to note that although the range in the encompassing volume (i.e. union of all definitions by one observer) was quite large, varying from 26.3 to $49.8 \mathrm{~cm}^{3}$, there was little variation in the common agreed upon volume (i.e. the union of all observations by an observer), the range being from 14.4 to $17.7 \mathrm{~cm}^{3}$ amongst the observers. 
The RCE for each of the six observers is also shown in table 4.1. The value of RCE ranges from 0.36 to 0.67 among all observers, indicating a large range in reproducibility within the observer group.

The overall systematic error in delineated volume is defined as the difference between the mean delineated volumes and the "true" gold standard volume. In our sample this was found to be $6.0 \mathrm{~cm}^{3}$. The source of systematic error is unknown. The inter-observer variation in prostate volume delineation is calculated as the standard deviation of the mean delineated volumes among six observers and was measured as $5.0 \mathrm{~cm}^{3}$. Similarly we found the intra-observer variation to be somewhat smaller at $1.8 \mathrm{~cm}^{3}$, indicating that the group variation (inter-observer variability) is significantly larger than the selfdeference (intra-observer variability).

Table 4.1 mean prostate volume, encompassing volume and common agreed volumes $\left(\mathrm{cm}^{3}\right)$

\begin{tabular}{||c|c|c|c|c|c|c||}
\hline & Observer 1 & Observer 2 & Observer 3 & Observer 4 & Observer 5 & Observer 6 \\
\hline Mean volume & $31.0 \pm 2.5$ & $21.7 \pm 1.4$ & $33.0 \pm 1.4$ & $23.9 \pm 1.9$ & $21.1 \pm 0.4$ & $27.8 \pm 2.2$ \\
\hline Encompassing & 49.8 & 30.5 & 42.9 & 40.0 & 26.3 & 41.8 \\
\hline $\begin{array}{c}\text { Common } \\
\text { agreed }\end{array}$ & 17.7 & 14.4 & 15.7 & 15.8 & 17.6 & 16.9 \\
\hline RCE & 0.36 & 0.47 & 0.37 & 0.40 & 0.67 & 0.40 \\
\hline$f_{T I}(100 \%)$ & $87 \pm 2$ & $80 \pm 3$ & $87 \pm 2$ & $84 \pm 2$ & $79 \pm 1$ & $83 \pm 3$ \\
\hline \hline
\end{tabular}




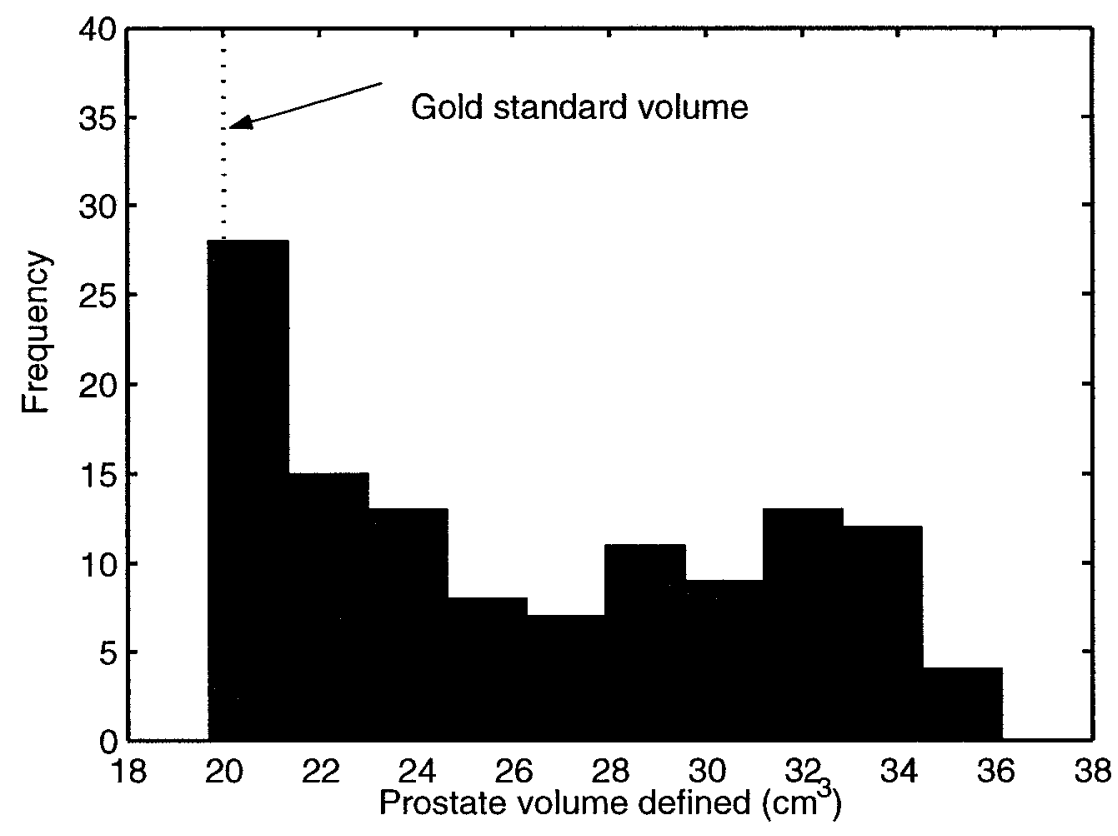

Figure 4.7 Histogram of the delineated prostate volumes

Figure 4.7 is a histogram of the delineated volumes while figure 4.8 is a histogram showing the distribution in percentage of gold standard volume included in each of the $120 \mathrm{CT}$ volume definitions from the six observers. It can be seen that in almost every case the observers define a prostate volume that is larger than the "true" volume of 20.4 $\mathrm{cm}^{3}$, the average defined volume being $26.4 \mathrm{~cm}^{3}$ with a range of 20.0 to $36.1 \mathrm{~cm}^{3}$. The ratio of delineated to true volume, $1.29(\sigma=0.25)$, is significantly different from unity with $\mathrm{p}<0.005$. Although the volumes defined by the observers are consistently larger than the gold standard volume, we see in Figure 4.8 that the percentage of true volume encompassed is always less than $100 \%$, with an average of $83 \%$ and a range from $74 \%$ to $91 \%$. The variation in the true prostate volume agreement $(3 \%)$ is less than that in CT delineated volume (18\%). 


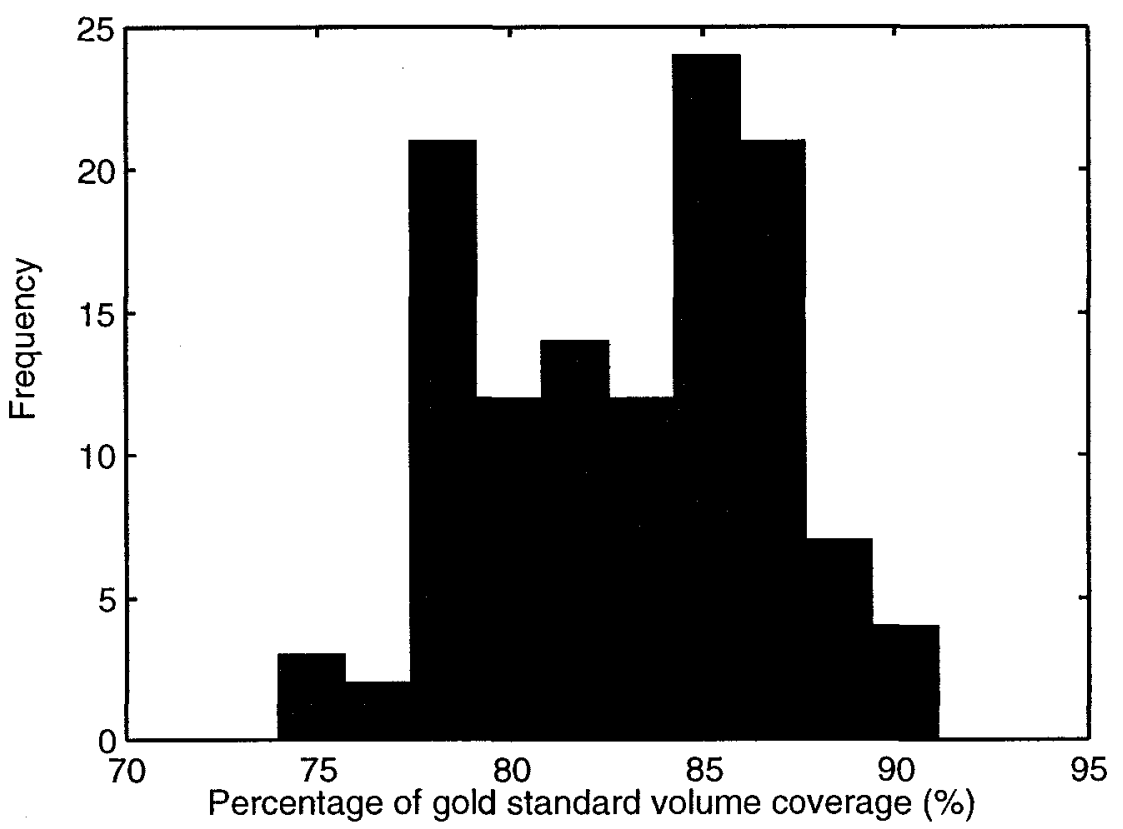

Figure 4.8 Histogram of the percentage of true volume included in each delineation

Table 4.2 Geometric displacements along LR, AP and SI directions

\begin{tabular}{|c|c|c|c|}
\hline & LR & AP & SI \\
\hline Mean GC shift & $0.4 \mathrm{~mm}$ & $3.9 \mathrm{~mm}$ & $3.0 \mathrm{~mm}$ \\
\hline Intra-observer variation & $0.3 \mathrm{~mm}$ & $0.5 \mathrm{~mm}$ & $1 \mathrm{~mm}$ \\
\hline Inter-observer variation & $0.6 \mathrm{~mm}$ & $0.7 \mathrm{~mm}$ & $1.4 \mathrm{~mm}$ \\
\hline
\end{tabular}

The geometric centre of each CT delineated prostate volume was calculated and compared to the geometric centre of the true prostate volume. The resulting difference represents a spatial displacement of the delineated prostate volume from its actual location in LR, AP and SI directions. The mean and standard deviation of the calculated displacements in the three principal directions are plotted in the Figure 4.9 for each of the 6 observers. As seen in this figure, the geometric centre of the CT prostate volume is shifted anteriorly a mean distance of $3.9 \mathrm{~mm}$ with a range of 1.8 to $5.4 \mathrm{~mm}$. In contrast, the displacement of the geometric centre laterally is very small, having a mean of $0.3 \mathrm{~mm}$ and a total range from -1.3 to $1.6 \mathrm{~mm}$. The mean displacement of the geometric centre of 
the delineated CT volumes and the inter- $\&$ intra observer variation are summarized in the table 4.2.

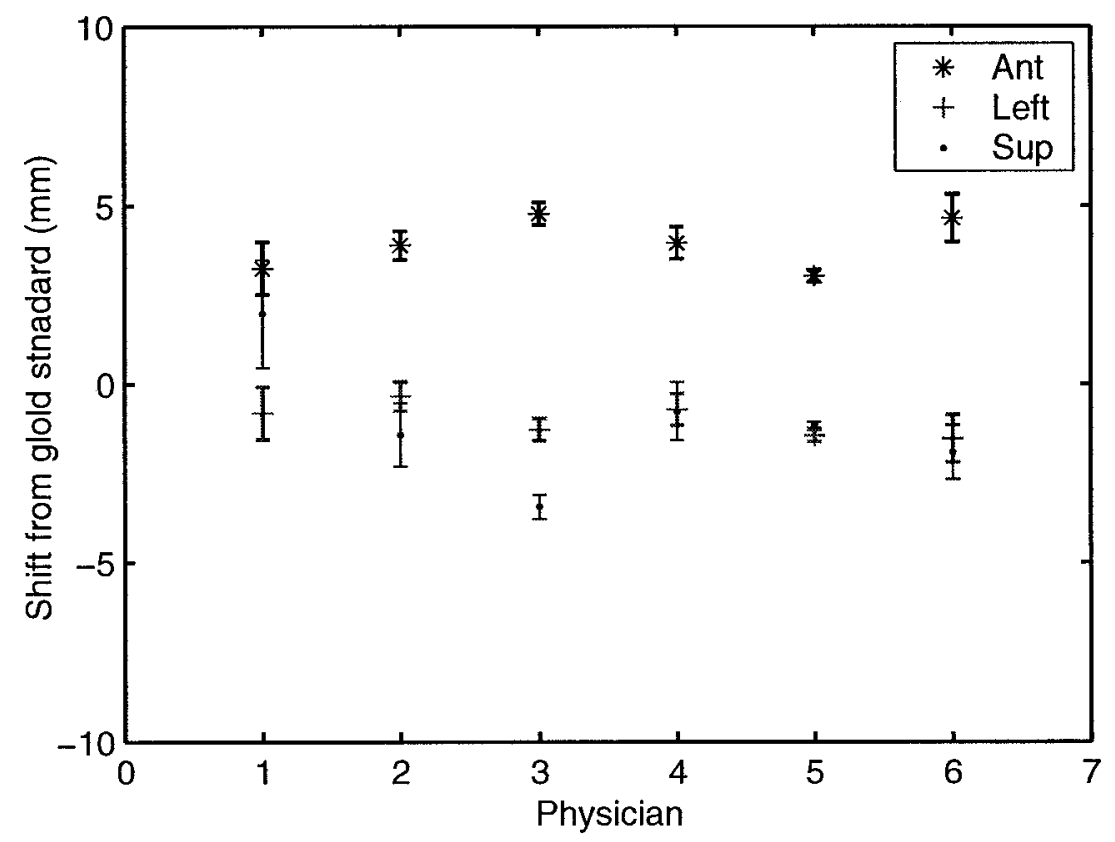

Figure 4.9 Centroid displacements in three orthogonal directions

The gap between the oncologist-delineated contours and the true prostate contours for each of the four quadrants was also measured. The random uncertainty, the systematic error, and inter- and intra-observer uncertainty in the gap for each of the four quadrants are summarized in table 4.3. As can be seen, on average the radiation oncologists missed $2.8 \mathrm{~mm}$ of the posterior portion of the prostate while anteriorly $5.8 \mathrm{~mm}$ of normal tissue was included as target. The large systematic error of $-2.8 \mathrm{~mm}$, coupled with quite small inter and intra observer variability in posterior direction suggest that physicians are more concerned about incorrectly including rectum than missing target. Conversely we observe that anteriorly there is a large +ve systematic error and a large random error, suggesting that physicians are much less concerned about incorrectly including bladder than they are about missing target tissue anteriorly. 
Figure $4.10 \mathrm{a}, \mathrm{b}, \mathrm{c}, \mathrm{d}$ are plots of the mean and standard deviation of the gap as a function of the prostate image slice number from the base to apex for the four cardinal directions. These suggest that the gap between true and delineated prostate is directionally dependent. There is a systematic miss posteriorly and significant amounts of normal tissue falsely included anteriorly. The figures also indicated that the overall variability is smaller in the middle of the true prostate than at the ends.

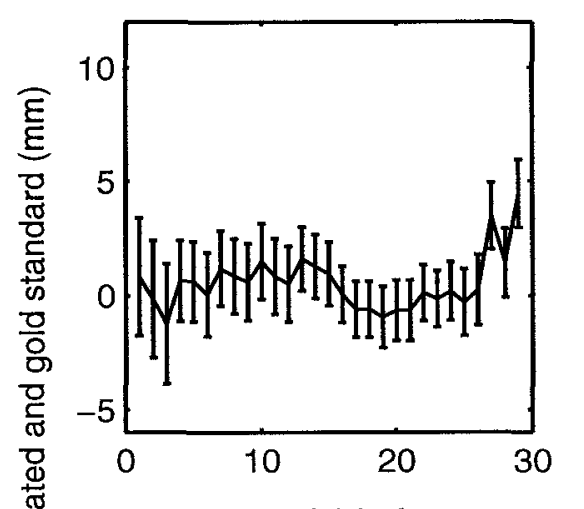

(a) Left

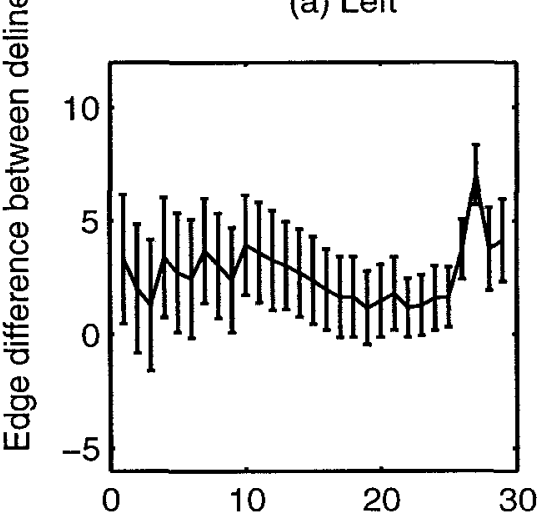

(c) Right

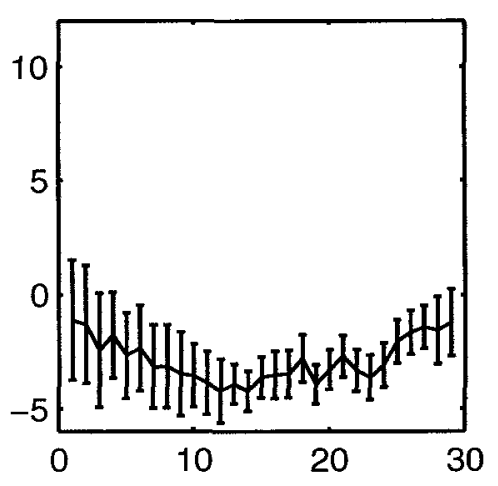

(b) Post

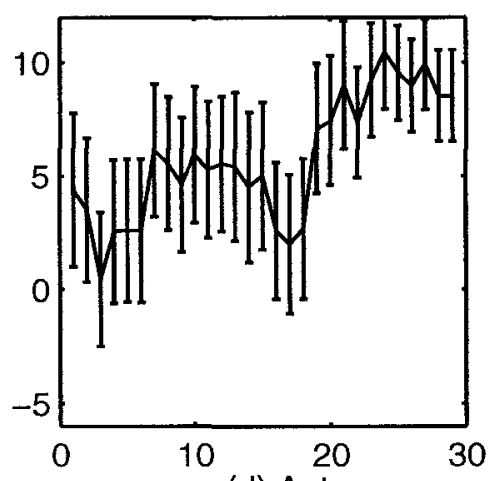

(d) Ant

Prostate inferior-superior axis $(\mathrm{mm})$

Figure 4.10 The mean and standard deviation of the gap as a function of prostate slices from base to apex for four quadrants (a) left, (b) posterior, (c) right and (d) anterior

Table 4.3 Gaps ( $\mathrm{mm})$ between delineations and gold standard in four quadrants

\begin{tabular}{|c|c|c|c|c|}
\hline Error Types & Left & Post & Right & Ant \\
\hline Random uncertainty & 2.0 & 1.8 & 2.4 & 3.9 \\
\hline Systematic error & 0.6 & -2.8 & 2.7 & 5.8 \\
\hline Inter-observer uncertainty. & 0.7 & 0.5 & 1.5 & 2.0 \\
\hline Intra-observer uncertainty & 0.5 & 0.5 & 0.6 & 0.7 \\
\hline
\end{tabular}


The Probability Volume Histograms (PVH) in each of the four quadrants are plotted in figures 4.11 and Figure 4.12 for both the prostate and non-prostate volumes, respectively. As can be seen in Figure 4.11, the oncologists included $77 \%$ of anterior quadrant volume $\left(5000 \mathrm{~mm}^{3}\right) 100 \%$ of the time, that is in each of 120 delineations. Posteriorly this changes dramatically, where only $20 \%$ of the true volume $\left(4800 \mathrm{~mm}^{3}\right)$ is included $100 \%$ of the time. Our quadrant volume data also indicates that a portion of true target volume (approximate $450 \mathrm{~mm}^{3}$ ) in the posterior quadrant is never included in any of the 120 delineations. We further divided the true prostate volume into superior, middle and inferior parts (each section is covered by 11 photo slices). The quadrant based PVH analysis shows that $55 \%\left(250 \mathrm{~mm}^{3}\right)$ of the volume that was always missed lay in the superior third of the prostate. Figure 4.12 shows the PVHs for normal tissues that are incorrectly defined as target tissue. In the figure, it can be seen that there is a significant difference in this error depending on the direction. Anteriorly, a portion of the bladder $\left(1100 \mathrm{~mm}^{3}\right)$ that is always included as target. It can also be seen in Figure 4.12 that a total volume of $15500 \mathrm{~mm}^{3}$ of bladder is included at least once in the physiciandelineated volumes. In comparison, only $4000 \mathrm{~mm}^{3}$ of rectal volume is included at least once in physician-delineated volume and no part of the rectum was included in all 120 target delineations.

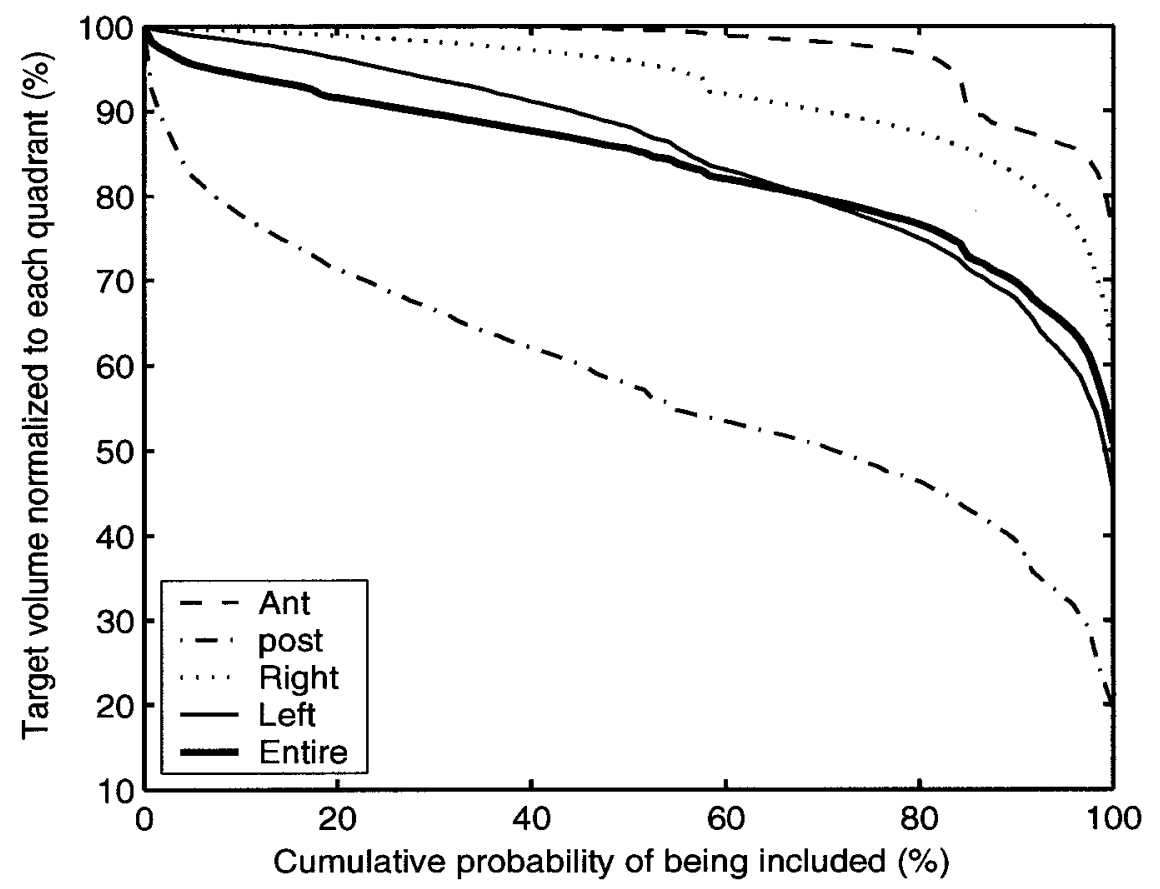

Figure 4.11 Volume definition histogram for prostate target for four quadrants 


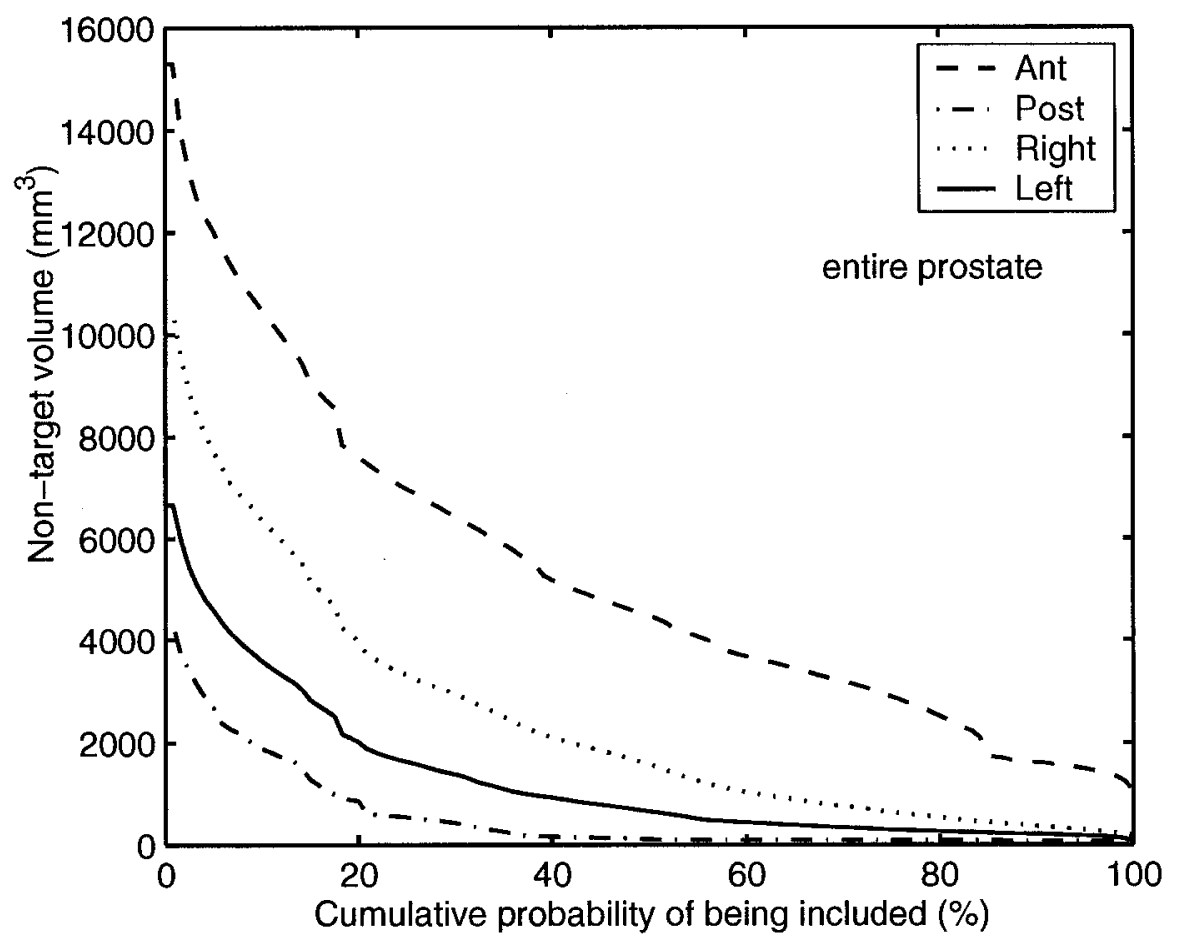

Figure 4.12 Volume definition histogram for non-target volume for four quadrants

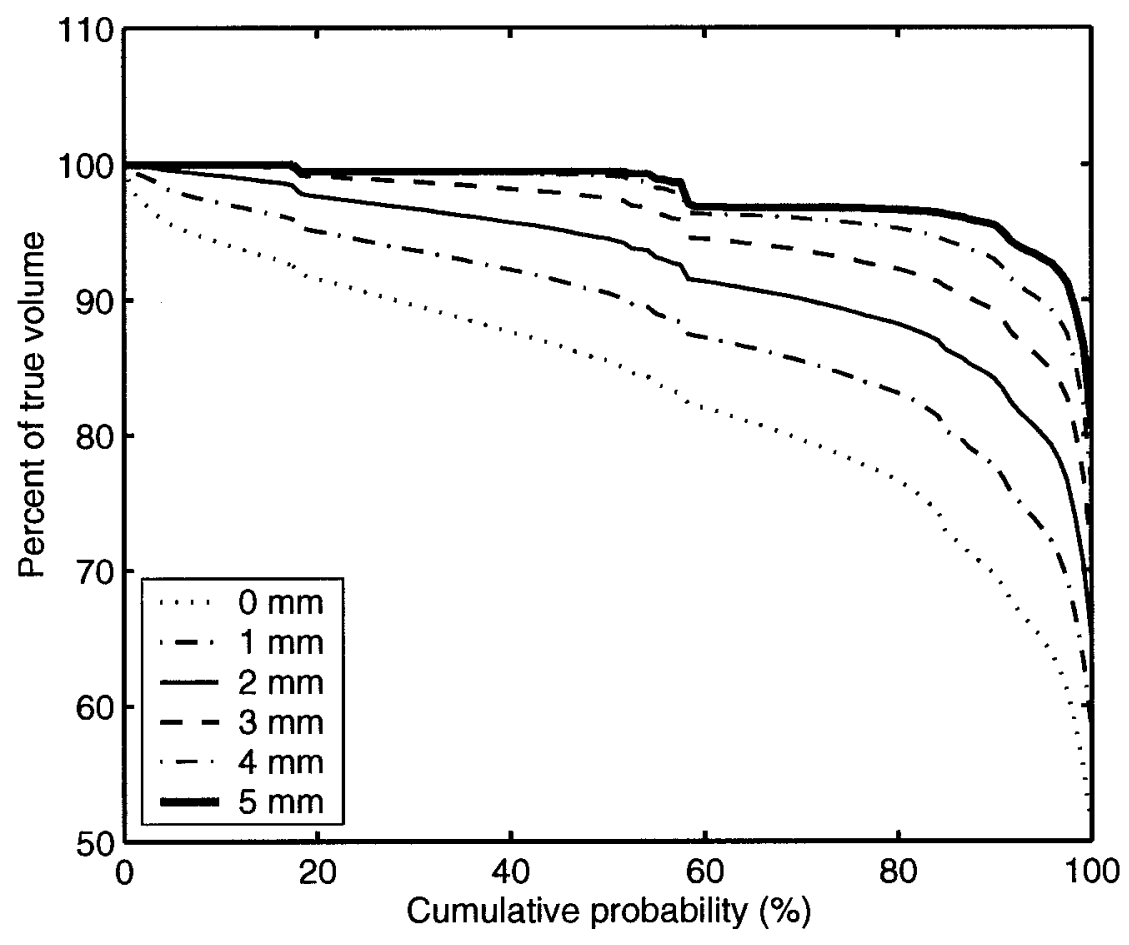

Figure 4.13 PVH for different margins 
Clinically, it is important to ensure tumour coverage and it is particularly important to minimize systematic errors. Clearly target delineation errors propagate from the treatment preparation stage through treatment as systematic errors. We introduce here the idea of a CTV contouring margin to account for uncertainties in segmentation, similar to the PTV margin which is proposed to address geometric setup and organ motion errors. Figure 4.13 shows the family of PVH curves for the entire prostate if an isotropic margin varying between 1 and $5 \mathrm{~mm}$ is applied. As can be seen in the figure, a $5 \mathrm{~mm}$ margin added to the delineated volume can include more than $95 \%$ of the true volume with a probability of $90 \%$, but an unnecessary amount of bladder is treated.

\section{4 Discussions}

\subsubsection{Study limitations}

In this chapter, we have investigated inter- and intra- observer variability in prostate target delineation. More importantly, we have also measured the individual and group observer agreement with the true prostate volume, where the true prostate volume was determined from anatomical photographic slices of the visible human male. In previous prostate delineation studies, ${ }^{[27,31,33,132]}$ it has not been possible to extract systematic errors or directional bias because the "true" prostate has been accepted as the average of the population observations and thus only variance can be measured. By taking advantages of both CT and anatomical data of a male cadaver from the VHP project, we can not only measure the reproducibility of target delineation (e.g. inter- and intra- observer variation target delineation), but also the systematic error for the CT delineated prostate target. To our knowledge, this is the first study to measure the absolute difference between defined and true volume for a human subject.

The performance of the prostate target delineation is in general affected by the uncertainty in patient, modality and observer ${ }^{[24]}$. Because only one patient subject and one modality were examined in this study, the effect of the inter-patient \& inter-modality variation was unavailable. 
The prostate organ of the VHP male (age 38) was healthy when it was imaged both through CT and photographic scanning. The prostate was much smaller than is usually seen in cancer clinics. The prostate volume of the visible human was measured to be 20.4 $\mathrm{cm}^{3}$, which is close to the average value of $25 \mathrm{~cm}^{3}$ for a healthy adult prostate, but is much less than the defined prostate PTV volume $\left(139 \sim 318 \mathrm{~cm}^{3}\right)$. The volume difference between healthy and cancerous prostate may affect the performance of the prostate delineation.

The CT images and photographic images of the VHP male were acquired twenty years ago, since then, CT technologies have been significantly improved in both the resolution and contrast. It has been accepted that image contrast can considerable affect the accuracy and uncertainty of the organ delineation by a physician. Therefore the variation in prostate delineation on an old CT by a physician may not well represent that on a new CT.

\subsubsection{Comparisons with Other Studies}

The intra-observer and inter-observer difference in volume delineation on CT and MRI for prostate cancer have been investigated and quantified in several previous studies $[26,27,134]$. Based on prostate volume measurements for six patients from five radiation oncologists, Fionrino ${ }^{[27]}$ concluded that the intra-observer variability in volume delineation was relatively smaller $(\sim 5 \%)$ than the inter-observer variability $(10 \sim 18 \%)$. He also pointed out that the inter-observer variability is patient dependent. Our results are consistent with Fionrino's. In our study, the intra-observer difference ranged from 2 8\% for all observers, however, inter-observer difference is $18 \%$. As suggested by $\operatorname{Rasch}^{[26]}$, the ratio between the common agreed and encompassing volume describes the delineation uncertainty for an individual observer or population.

One aim of this study was to quantify the inter- and intra-observer variability within our department and further determine whether these inter-observer differences are systematic 
or random. We found that there was a systematic displacement of geometric centre from true prostate volume in anterior direction. Several factors could cause this systematic displacement. The quality of the CT image might not be good enough for accurate segmentation of prostate from adjacent organs such as rectum and bladder. The normal tissue encompassing and prostate missing in a prostate delineation could also change geometric centre location. We also suspect that the participating radiation oncologists

specifically avoided inclusion of rectal volume due to its radiation sensitive. Considering a tight margin for prostate cancer in posterior direction $(5 \sim 10 \mathrm{~mm})$ and a systematic error $(-3.9 \mathrm{~cm}$ shift to anterior direction) in target delineation, the current margin in our centre may be too conservative.

\subsubsection{Margin Optimization}

It has been accepted that tumour control probability depends not only on accurate dose delivery but also on accurate target delineation. The dose to a planned target can be verified during or after radiation delivery. However, it is not possible to verify whether a delineated volume represents or includes the entire true tumour volume. Without knowing the true tumour target, it is difficult to estimate tumour control probability. In order to increase the chance of including the entire tumour volume in the target delineation, a margin should be applied to the delineated volume. An optimized margin can be obtained to include the true target volume at some confidence level. We have added 5,10 and $15 \mathrm{~mm}$ margin to each of $120 \mathrm{CT}$ delineated volumes in their axial and transverse directions. We found that 105 of the 120 physician-delineated volumes included the entire true volume if a margin of $5 \mathrm{~mm}$ is applied around the delineation. Further, a $10 \mathrm{~mm}$ margin ensures inclusion of the entire true prostate organ $100 \%$ of the time. Enlarging the prostate delineation increases the risk of including more rectal and bladder tissue. Comparing the three different margins, we found that margins of 5,10 and $15 \mathrm{~mm}$ result in an increase in rectal volume of $2 \%, 15 \%$, and $30 \%$, respectively. Introducing a uniform margin around the delineated prostate to mitigate delineation uncertainty might be inappropriate because that the gap uncertainty is radialy dependant. A margin of $5.6 \mathrm{~mm}$ (or two times the systematic gap error in posterior direction) is 
required posteriorly, while a smaller or zero margin is required anteriorly. For our 6 field 3DCRT, the margin around the CTV in the posterior direction is $5 \mathrm{~mm}$ for boost treatment. Considering a margin of $12.7 \mathrm{~mm}$ is required to mitigate against the overall geometric uncertainty during treatment course ${ }^{[103]}$ We conclude that our current margin of $5 \mathrm{~mm}$ for boost treatment in posterior direction may be inadequate.

\subsection{Conclusions}

Several conclusions can be made from this study:

The physician-delineated prostate volume on CT is on average $30 \%$ larger than the volume of the true prostate. The agreement between CT delineated and true prostate volumes was used to describe the goodness of the prostate definition. We have found that a CT prostate definition on average only includes $84 \%( \pm 3 \%)$ of the true prostate volume, while encompassing $9 \mathrm{~cm}^{3}$ of normal tissues. By applying a uniform margin of $5 \mathrm{~mm}$ around delineated prostate target, the entire true prostate would be encompassed for $87 \%$ of all fractions.

There was a systematic difference between the geometric centre of the volumes defined by the physicians and the gold standard volume. This was $3.9 \mathrm{~mm}$ anterior to the gold standard geometric centre. It appears that the radiation oncologists are more fearful of including rectum than of missing tumour posteriorly, while in contrast they are more worried about missing tumour anteriorly than accidentally including bladder.. This is also seen in our gap analysis. We found that the posterior edge of the physician defined prostate was on average $2.8 \mathrm{~mm}$ short of the gold standard. At the same time the physician defined anterior border extended an average of $5.8 \mathrm{~mm}$ anterior to the anterior border of the gold standard prostate. The boost phase of our 3DCRT prostate treatment currently uses a margin of $5 \mathrm{~mm}$ posteriorly. Based on our delineation data, we feel that this margin may be too small and should be reviewed. 
In general inter-observer variation is larger than in intra-observer variation for geometric centre and prostate volume. This reflects that self-deference in target delineation is small comparing to the group deference. The intra-observer variation was due to the inconsistency of the contrast setting while inter-observer variation is due to the experience, training and department delineation protocols among different observers. 


\section{CHAPTER 5 \\ Quantifying Independent Geometric Uncertainties in Prostate Cancer Radiotherapy}

\subsection{Overview}

This chapter describes the work, performed at The Ottawa Hospital Regional Cancer Centre (TOHRCC) from Sept. 2003 to June 2004, which investigates independent geometric uncertainties in prostate cancer External Beam Radiation Therapy (EBRT). Section 5.1 briefly introduces the various sources of geometric uncertainty associated with prostate cancer radiotherapy. Section 5.2 describes our approach to the measurement and quantification of these geometric uncertainties and section 5.3 summarizes our findings with respect to these errors. Finally section 5.4 discusses how our findings might be reflected in the clinical environment and how these findings may be implemented to positively affect treatment accuracy.

\subsection{Introduction}

The radiation therapy community commonly accepts that treatment outcomes for prostate cancer may improve if higher doses of radiation are delivered to the tumour while simultaneously sparing the adjacent normal tissues. Several clinical prostate studies have demonstrated a correlation between local tumour control and the total dose delivered ${ }^{[135-}$ ${ }^{137]}$ by showing that there is a statistically significant difference in biochemical (Prostate Specific Antigen) relapse-free survival in patients who receive $\geq 75.6$ Gy compared to those who received $\leq 64.8$ Gy. Unfortunately, dose escalation is difficult due to normal tissue toxicity. The ability to limit normal tissue dose is compromised by the need to provide a geometric margin around the target. This margin is dictated by a variety of 
geometric uncertainties that arise during various stages of the overall treatment process. These include target definition, inter and intra fraction patient motion, organ motion, daily positioning error and linac geometric uncertainties such as isocentre (laser) alignment and collimator jaw/leaf calibration. Geometric uncertainties are mitigated by enlarging the volume of tissue treated. ${ }^{[19,20]}$ Several recipes suggested by different investigators ${ }^{[50,100-102]}$ have been published to optimize the treatment margin. Currently there are two approaches to defining a margin around the CTV: the uniform approach simply adds a constant margin around the CTV while the non-uniform approach employs a directionally dependent margin. Because the uniform approach ignores the nonisotropic nature of geometric errors, it generally leads to unnecessary normal tissue exposure. Alternately the non-uniform approach requires knowledge of the total geometric error in any direction around the CTV. Recently published uncertainty data $[41,69,73,74]$ allows for the optimization of lateral and AP margins around the prostate. However, modern radiotherapy techniques such as 3DCRT or IMRT employ oblique beams that require radially dependent uncertainty data for margin optimization. Unfortunately, little oblique uncertainty data have been published for prostate radiotherapy.

\subsection{Materials and Methods}

\subsubsection{Study Population, Treatment Planning and Delivery}

A total of twenty-one early stage prostate cancer patients were randomly selected for this study, all patients receiving $76 \mathrm{~Gy}$ in 38 daily fractions consisting of an initial volume (56 Gy) and a boost volume (20 Gy). Fourteen of twenty-one patients were treated prone with Hip-Fix immobilization and seven were treated supine without immobilization. Patients were treated with empty bladders using $18 \mathrm{MV}$ photons from a Siemens Digital Mevatron (Siemens Oncology System, Concord, CA), the radiation portals being defined by a fifty-eight leaf MLC. 
Prior to treatment each patient's prostate was implanted with three gold fiducial markers ( $2 \mathrm{~mm}$ long, $1.1 \mathrm{~mm}$ diameter, Oroplato, Quebec, Canada) under ultrasound guidance. The seeds were nominally positioned at the base of the prostate near the seminal vesicles as well as the posterior aspect, and the apex. Following seed implantation, the patient had a planning CT and was planned for our conventional six-field XRT technique. The target volume (prostate plus seminal vesicles), the rectum and the bladder as well as the fiducial markers were contoured according to the TOHRCC protocol by the radiation oncologist. During dose delivery, daily portal images of each of the 4 oblique treatment portals were acquired using the Siemens Beamview TI (Siemens Oncology System, Concord, CA) electronic portal imager. In total 1028 images were acquired, representing 257 treatment fractions ( 4 images per fraction) from 21 patients during the initial phase (first $56 \mathrm{~Gy}$ ) of treatment. The images were used to determine the global geometric errors and independent geometric errors (e.g. patient positioning error and internal organ motion) over the course of treatment. The portal image data were not used for clinical corrections.

\subsubsection{Image Enhancement and Correction for Spatial Distortion}

Analysis of our Siemens TI portal imager revealed several problems that needed to be addressed before the images could be used for feature extraction and localization. It was determined that there was substantial but non-consistent mechanical displacement of the portal imager as a function of gantry angle. In addition there was significant spatial distortion of the image and a spatially dependent response (intensity gradient) across the imager. Finally, the raw images were almost unusable and required significant signal processing in order to extract anatomical features and the fiducial markers. The quality of a single raw image from the EPID was only sufficient to detect the portal field edges. The inherent low contrast made it impossible to extract either fudical markers or bony landmarks. The image quality was enhanced by optimizing the EPID setup and integration time and by averaging multiple consecutive frames. The subsequent images were improved but still not sufficient to extract the data needed for this study. Consequently the contrast of images was enhanced using MHF (Mexican Hat function) 
method ${ }^{[76]}$. By selecting different kernels, either the contrast of the fudical seeds or selected bony landmarks could be enhanced. The detail of MHF imaging processing technique is reported in detail in chapter 3.

In order to correct for the spatial distortion of the images, an image-to-physical space transform was develop to correct the raw images. The correction method for geometric distortion is described in detail in chapter 3.

\subsubsection{Isocentre Determination}

An essential component of this work was to determine the displacement of an image with respect to the beam isocentre (axis of rotation of the collimator). It was initially hoped that the imager would be mechanically stable, or at least have a predictable position as a function of gantry angle such that beam isocentre could be associated with a specific pixel index. However, the portal imager was found to have significant daily variation in mechanical displacement with respect to the beam isocentre. On any given day there was also an unpredictable variation with respect to gantry angle and hence a simple predetermined mapping function or look-up table was not possible.

Normalized cross correlation and interleaf leakage analysis were then developed to determine the $\mathrm{X}$ and $\mathrm{Y}$ component of the beam isocentre in the portal image, respectively. These two methods are described in detail in chapter 3.

\subsubsection{Extraction of Intra-fraction Organ Motion}

Motion of the prostate during an individual treatment fraction is referred to as intrafraction organ motion. In general it is not possible to acquire this information directly from the low contrast planar images acquired with the Siemens TI portal imager. In this work, we approximate this motion by the difference in prostate position (surrogated by the centroid of three fiducial seeds) with respect to a fixed geometric point in two opposed fields taken in the same fraction as described below. 
In each fraction, the position of the prostate is manually determined offline with respect to the isocentre in each of the four oblique portals. In our case the oblique portals are the Left Posterior Oblique (LPO), the Left Anterior Oblique (LAO) the Right Anterior Oblique (RAO) and the Right Posterior Oblique. These are rotated $45^{\circ}$ from the cardinal angles and are opposed pairs such that the RAO and LPO are opposed, similarly the LAO and RPO. Motion is then detected in a co-ordinate frame $\left(x^{\prime}, y^{\prime}, z^{\prime}\right)$ rotated $45^{\circ}$ about the superior - inferior axis of the conventional IEC frame $(x, y, z)$ as shown in Figure 5.1. In TOHRCC six field prostate treatment technique, opposed oblique portals are identical when rotated $180^{\circ}$ about their superior-inferior axis. If the isocentre is located at (or very near) the fiducial COM then the displacement of the COM in the plane (XZ in Figure 5.1) between one image and its flipped opposed pair, represents the intra-fraction prostate motion that has occurred in the time between the acquisition of the two images.

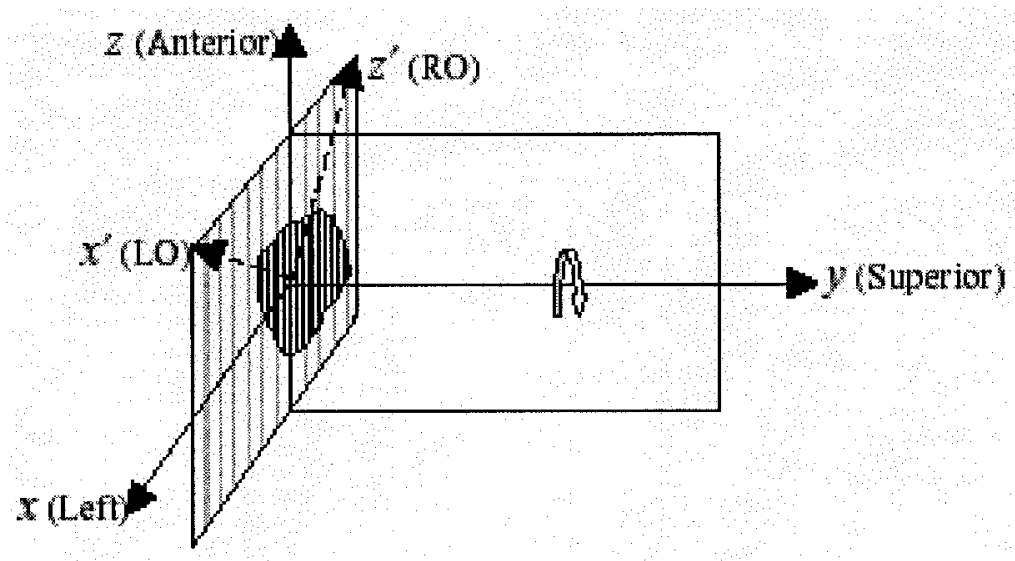

Figure 5.1 IEC and oblique frame 


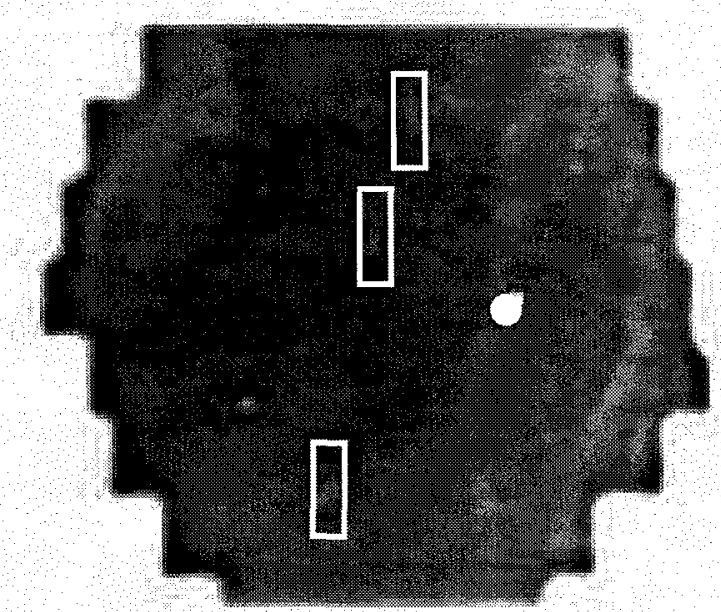

(a) RPO

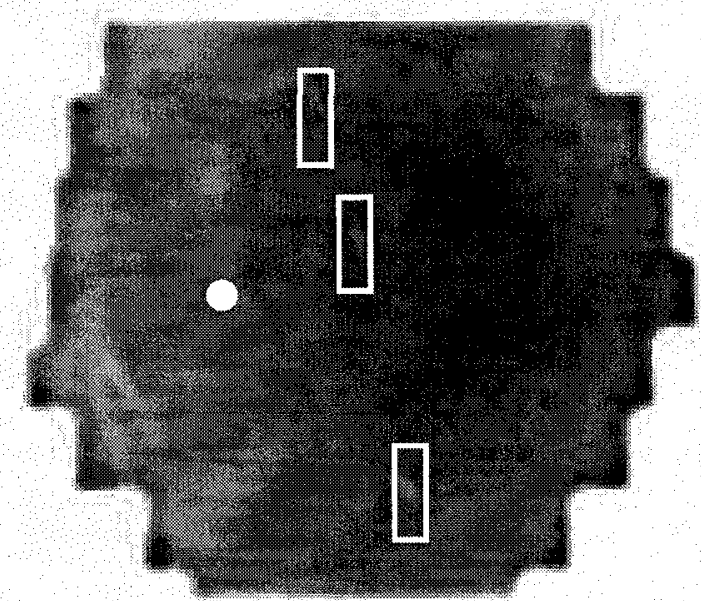

(c) RPO, flipped

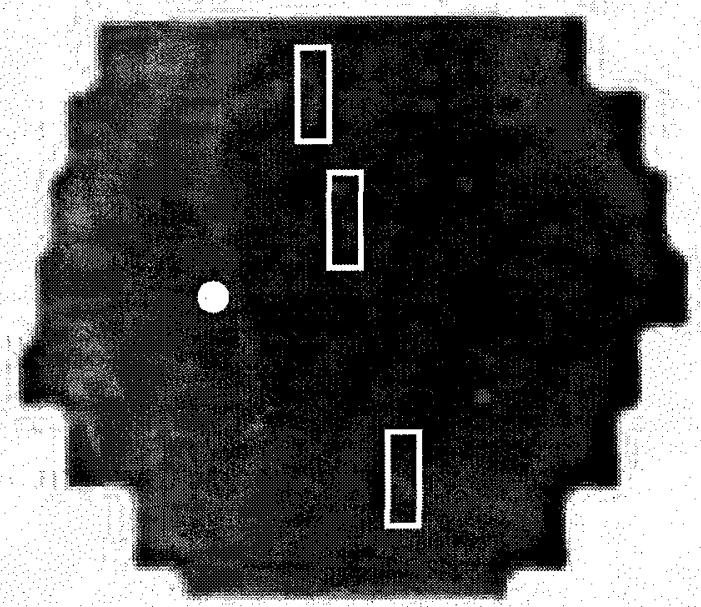

(b) LAO

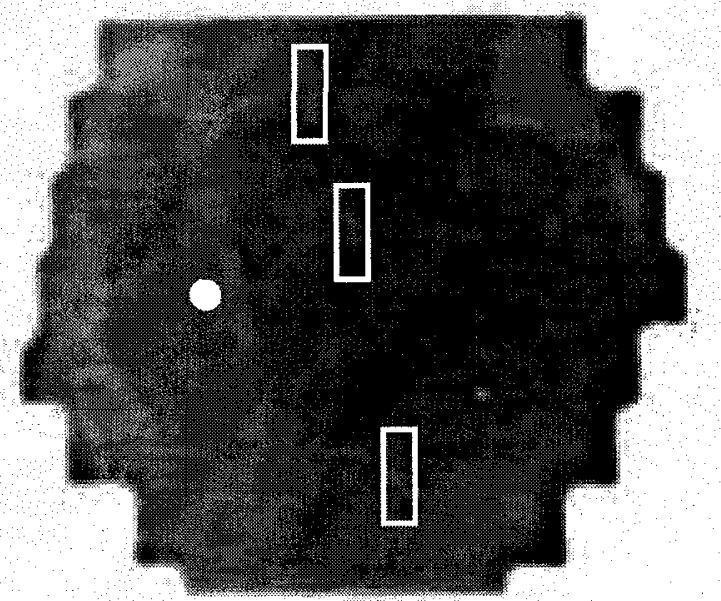

(d) LAO

Figure 5.2 Opposed image pairs used to determine intra-fraction prostate motion

As an example, Figure 5.2 illustrates two images (a and b) taken at gantry $45^{\circ}$ (RPO) and $225^{\circ}(\mathrm{LAO})$ for a prone patient. The portal shape of the two original images is identical if one of two images, say the RPO is horizontally flipped as shown if figures $5.2 \mathrm{c}$ and $\mathrm{d}$. For clarity, the three gold seeds are highlighted with boxes and the position of the isocentre is marked by a white dot. The shifts between the two sets of opposed image pairs were calculated for each of the 257 fractions and represents the intra-fraction organ motion along the SI and RO (or $\mathrm{LO}$ ) directions. 


\subsubsection{Determination of the Global Geometric Error}

The global geometric error is defined as the daily deviation of the prostate from its planned position with respect to field edges (or beam isocentre) for each specified field. In this work, we denote the global geometric error during fraction $i$, as $\Delta r_{i}^{t, p}$, and define it as the difference between $r_{i}^{t}$ and $r^{p}$ where $r_{i}^{t}$ is the fudicial marker Centre of Mass (COM) during delivery and $r^{p}$ is its intended position from the CT based treatment plan, such that:

$$
\Delta r_{i}^{t, p}=r_{i}^{t}-r^{p}
$$

The three dimensional position of the prostate (as surrogated by the COM of the seeds) can be determined by triangulation using orthogonal fields (e.g. LPO-LAO or LPO-RPO) if there is no prostate motion between the two images. However the images of the two fields do have a temporal separation of approximately 1 minute, and considerable total motion (patient, prostate) is known to occur between the delivery of any two fields within a treatment fraction. This intra-fraction prostate motion, if ignored will propagate itself into the triangulation, introducing error. We have found less fitting error if we average the COM position between opposed fields (e.g. LPO-RAO, LAO-RPO) and use these coordinates, $\left(x^{\prime}, y^{\prime}\right)$ and $\left(z^{\prime}, y^{\prime}\right)$ for the triangulation to determine the COM co-ordinates in a frame $\left(x^{\prime}, y^{\prime}, z^{\prime}\right)$ rotated $45^{\circ}$ about the sup-inf axis $(y)$ of the conventional $(x, y, z)$ IEC frame. The planned COM position, $\left(x_{p}^{\prime}, y_{p}^{\prime}, z_{p}^{\prime}\right)$ in the same frame is computed from the CT planning data and is used as the reference condition from which global geometric errors are calculated. The resulting deviations represent the total treatment error (geometric sum) including daily positioning error, inter-fraction target motion, MLC positioning error and isocentre laser marker error. 


\subsubsection{Determination of Patient Positioning Error}

As discussed in chapter 2, patient-positioning error is defined as the difference in position of the patient bony anatomy with respect to beam edge between two fractions. In this work, portal images are enhanced by convolution with an appropriate MHF kernel allowing bony landmarks within the pelvis to be identified. After reviewing all portal images acquired from the same field for an individual patient, a segment containing a bony landmark structure that is common to all images for the same field (e.g. RPO) for a specific patient was selected. The locations of the segment in each image are then determined using normalized cross correlation between the reference segment and each image. The daily variation in the position of the pelvis (surrogated by the a segment of bony landmark) relative to the isocentre is then defined as the random positioning error for the patient. In theory, this method could provide the estimation of systematic positioning error by comparing the segment location in a portal image against that in the planning Digital Reconstructed Radiographs or DRR's. However, this cannot be accomplished at our centre due to several factors. The slice thickness of the CT images acquired at TOHRCC is $3 \mathrm{~mm}$ and hence the generated DRR image does not provide a spatial resolution which is comparable to the portal image. In addition, there is a significant difference in the contrast of the DRR and the portal images arising from the difference in their apparent radiological densities at the two different energies at which they are acquired. It is thus very difficult to accurately identify and extract a bony structure from a DRR using a reference segment from a portal image. Because we cannot directly compare to the DRR, in this work we define the reference position for a patient setup to be the average of bony structure positions over all observed fractions for the patient. Subsequently, daily positioning error $\omega$ is the difference between the observed

position of bony structure and its mean $\bar{\omega}$. We describe the distribution of the positioning errors in the population by the standard deviation $\sigma_{P E}$, given as:

$$
\sigma_{P E}=\sqrt{\sum_{j=1}^{n_{p}} \sigma_{P E}^{2}(j) / n_{p}},
$$


where $\sigma_{P E}(j)$ is the standard deviation of the positioning errors for patient $\mathrm{j}$.

\subsubsection{Determination of Inter-fraction Organ Motion}

For each patient, as described in chapter 2 , the target positioning error $\zeta$ is considered to be the daily deviation of the prostate from its mean position over all observed fractions. Generally speaking, the target positioning error results from inter-fraction organ motion and patient positioning error. Inter-fraction organ motion is the difference in prostate position (surrogated by COM of seeds) with respect to the pelvis (surrogated by bone structure) between a fraction and the mean of all fractions. Since the positioning error is independently determined by image analysis of extracted bony structures, the interfraction organ motion can then be separated from the target positioning error $\zeta$ by subtracting the positioning error $\omega$, if we assume that both inter-fraction organ motion and positioning error have mean of zero.

Inter-fraction organ motion is then expressed as

$$
\delta=\varsigma-\omega
$$

The standard deviation of the inter-fraction organ motion for an individual patient and population can be calculated using a method similar to that for positioning error.

\subsubsection{Determination of Collimator Calibration Error}

Although radiation delivery equipment (e.g. linear accelerator or MLC) related error is small in amplitude, it can still contribute considerable uncertainty to the overall dose distribution. In this study, the uncertainties in isocentre and MLC/Jaws are investigated. The linac isocentre is defined as the intersection of the axes of rotation of the couch, the gantry and the collimator of a linac. At TOHRCC, the QA procedure measures the laser defined isocentre daily to ensure that the isocentre is within its tolerance ( $1 \mathrm{~mm}$, radius). The MLC/Jaw positioning calibration is measured weekly and the positioning error is obtained by analyzing historical QA data. 


\subsubsection{Statistics}

As described in chapter 2 , the difference $\overline{\Delta r}$ between the mean observed prostate position $r_{i}^{t}$ during radiation delivery and anticipated position $r^{p}$ is the systematic error for an individual patient, and is denoted as

$$
\overline{\Delta r}=\sum_{i=1}^{n_{f}} \frac{r_{i}^{t}}{n_{f}}-r^{p}
$$

Similarly, the target positioning error $\varsigma_{i}^{t}$ is defined as the deviation of prostate $r_{i}^{t}$ from its mean position during radiation delivery

$$
\zeta_{i}^{t}=r_{i}^{t}-\sum_{i=1}^{n_{f}} \frac{r_{i}^{t}}{n_{f}}
$$

By rearranging Eq. 5.3, 5.4 and 5.5, the global geometric error can be rewritten as

$$
\Delta r_{i}^{t, p}=\overline{\Delta r}+\zeta_{i}^{t}=\overline{\Delta r}+\delta_{i}^{t}+\omega_{i}^{t}
$$

It should be noted that we assume, as reported in the literature ${ }^{[36]}$ that for a large sample size, the target positioning error $\varsigma^{t}$ is Gaussian with a mean of zero and a standard deviation of $\sigma\left(\xi^{t}\right)$. For an individual patient, $\sigma\left(\varsigma^{t}\right)$ can be approximated to be $\sigma\left(\varsigma^{t}, n_{f}\right)$, the corrected standard deviation for limited sample sizes, where $n_{f}$ is the number of observations (ranging from 7 to 20 in this study).

The global systematic error $M_{T}$ and the global geometric uncertainty $\sigma_{T}$ for all fractions in the patient population are calculated to be the mean and standard deviation of the global geometric errors $\Delta r^{t p}$ respectively, assuming that global geometric errors are not 
correlated to each other. The global systemic error $M_{T}$ is an indicator of global displacement for the entire patient population while the overall random uncertainty $\sigma_{T}$ describes the distribution of the global geometric error caused by a combination of individual systematic errors, inter-fraction organ motion and positioning error, both in the planning and delivery stages. The global geometric error also incorporates the MLC positioning error and mechanical isocentre uncertainty in this study.

According to van Herk, ${ }^{[68]}$ the global geometric error can also be categorized in terms of treatment preparation and treatment delivery uncertainties. Treatment preparation uncertainty, also referred to as the systematic uncertainty, for a group of patients is $\Sigma$ and is calculated to be the standard deviation of the individual systemic errors $\overline{\Delta r}$ while the treatment delivery uncertainty, or random uncertainty, $\sigma$, is calculated to be the quadratic average of the uncertainties of the target positioning error $\varsigma^{t}$ of all patients.

Similarly, inter-fraction organ motion, positioning error and intra-fraction organ motion can also be quantified, but in our work, as discussed above, we cannot determine components of systematic error for each geometric factor because we do not have a quality reference image of the planned patient position.

\subsection{Results and Analysis}

In total, 1028 electronic portal images were acquired during the delivery of 257 radiation treatment fractions (prone: 184 fractions, 14 patients; supine: 73 fractions, 7 patients). After correction for geometric distortion, the portal images were aligned to remove orientation and translation inconsistency. Images were subsequently analyzed as described above to estimate the geometric uncertainties from the different sources, and are reported here in the patient oblique frame of reference associated with the oblique fields used for prostate radiotherapy. 


\subsubsection{An Example of Positioning and Global Geometric Error for a Specific Patient}

In general, the position of the prostate as defined on the planning CT for is the reference for patient repositioning during radiation delivery. Thus, the deviation of the prostate position (surrogated by fiducial markers) with respect to the BEV represents the error due to a combination of many factors in the radiation treatment process including individual systematic error, inter-fraction organ motion and patient positioning and collimator calibration error. As an example, Figures 5.3(a) to 5.3(c) show bar plots of global geometric error in the patient oblique frame (LO, RO and SI axes) for 20 fractions of an individual patient's treatment. In addition each graph also shows the measured patient positioning or setup error as surrogated by bony landmarks. As discussed above setup error is with respect to the mean setup position and is therefore distributed about zero.

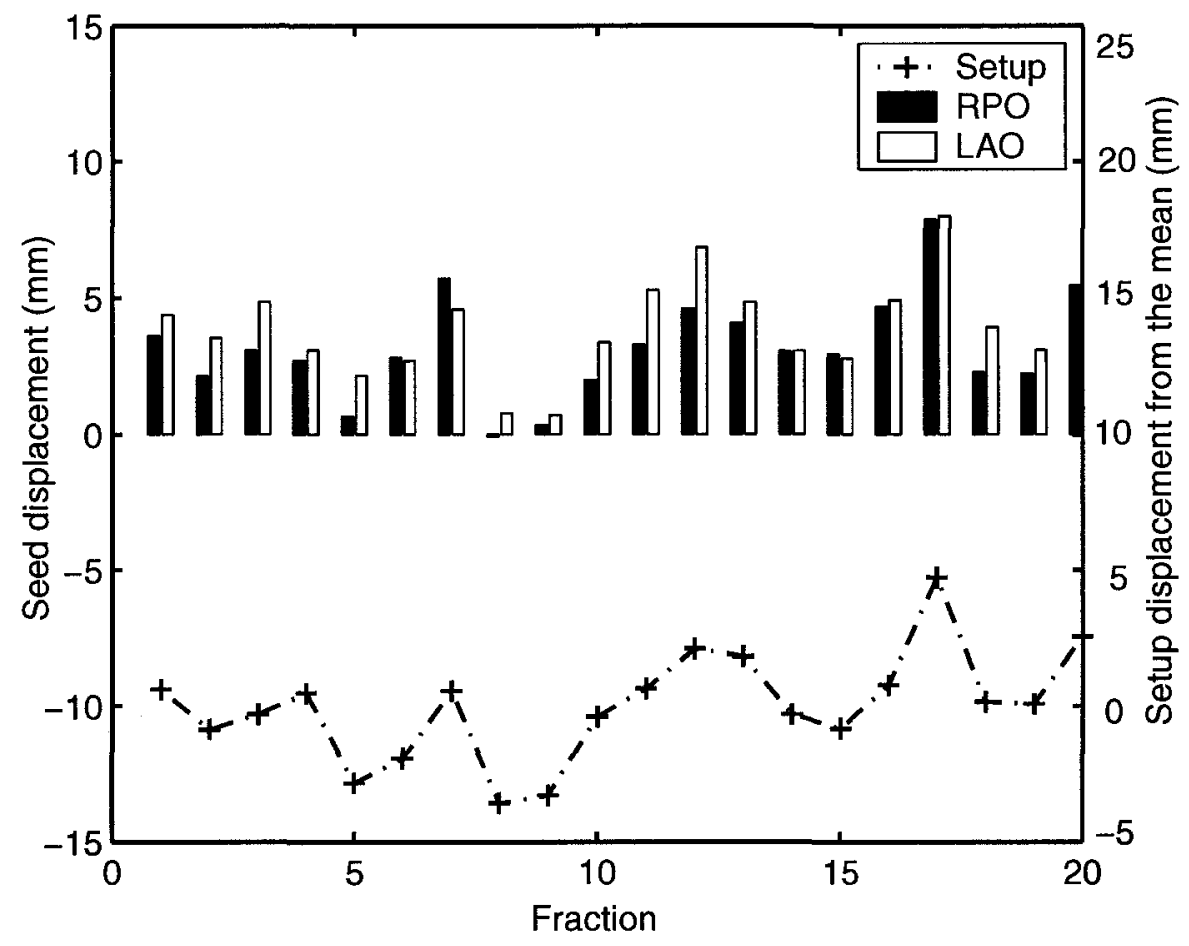

Figure 5.3(a) Global geometric error and positioning error in LO direction 


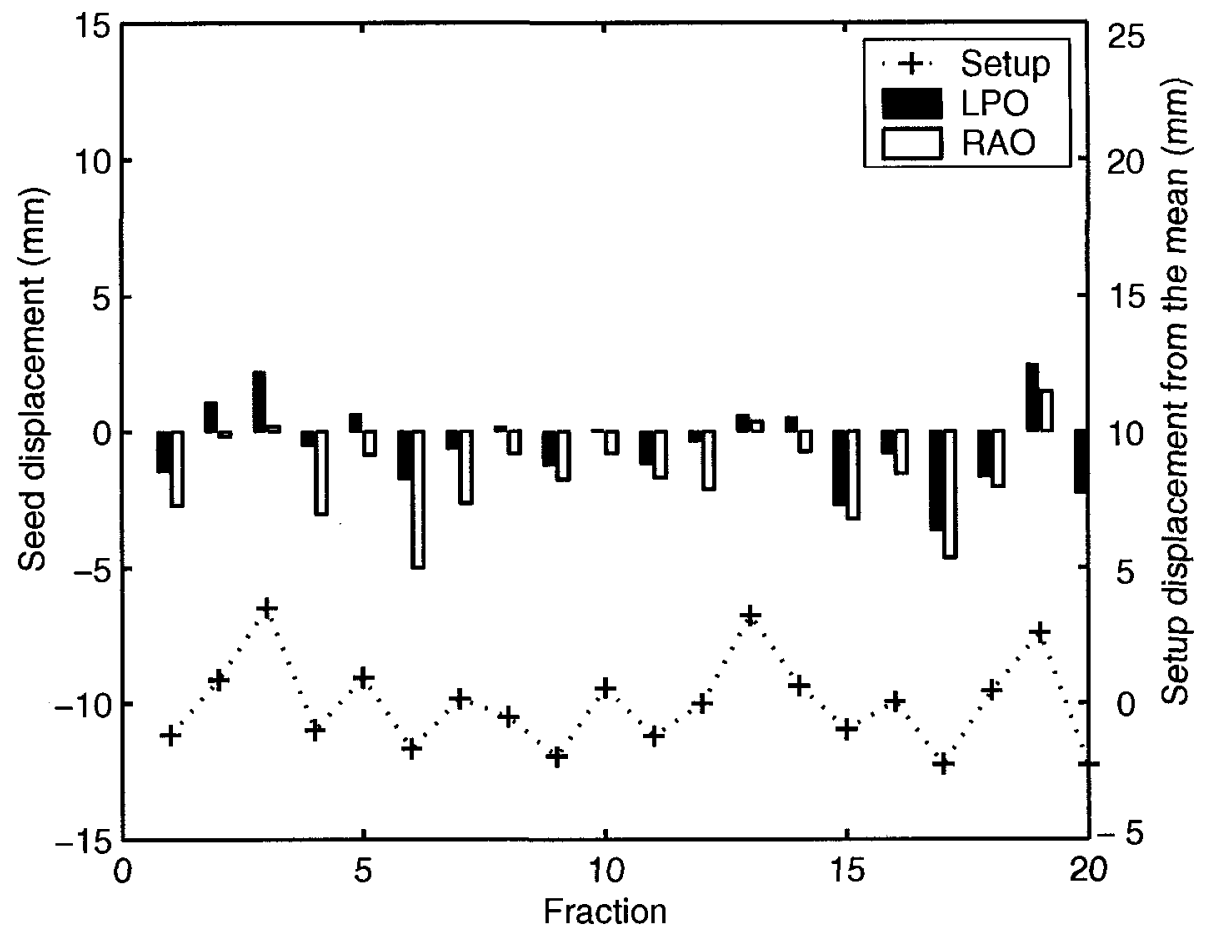

Figure 5.3(b) Global geometric error and positioning error in RO direction

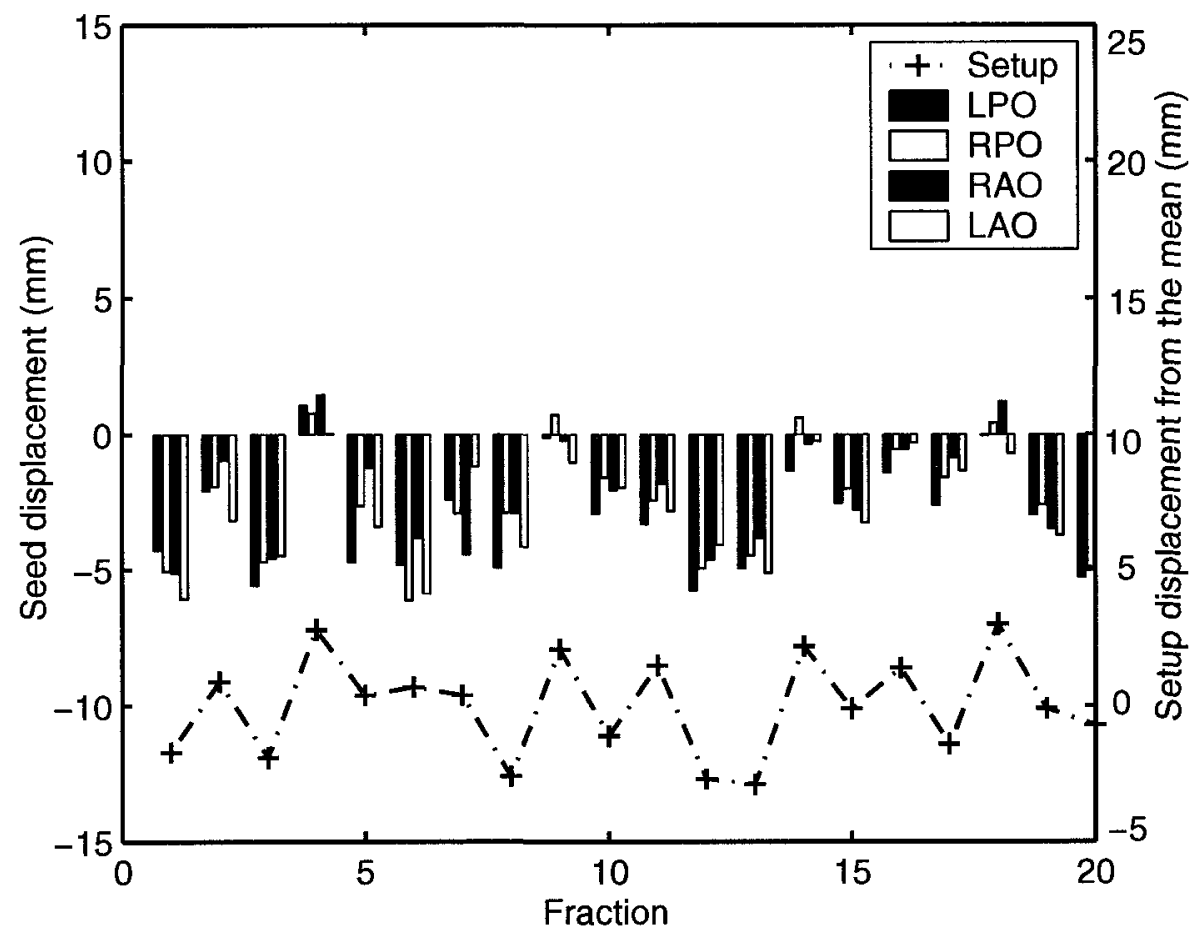

Figure 5.3(c). Global geometric error and positioning error in SI direction 
The mean $\overline{\Delta r}$ and standard deviations, $\sigma\left(\varsigma^{t}\right)$ of global geometric error for the patient whose data is shown in figures 5.3 (a),(b), (c) were found to be $-1.2 \pm 1.6 \mathrm{~mm}, 1.6 \pm 2.0$ $\mathrm{mm}$ and $3.6 \pm 1.9 \mathrm{~mm}$ in the $\mathrm{LO}, \mathrm{RO}$ and SI directions, respectively. Similarly the patient positioning uncertainties $\sigma_{P E}$ for this subject were found to be $1.8,2.0$ and $1.7 \mathrm{~mm}$. Following the decoupling of the patient positioning error from the target position as per Eq. 5.3, the standard deviation of inter-fraction organ motion was determined to be 1.8 , 1.6 , and $1.9 \mathrm{~mm}$ in SI, LO, and RO, respectively. The standard deviations were measured to be $0.9,0.8$ and $1.4 \mathrm{~mm}$ in LO, RO and SI directions, respectively.

\subsubsection{Data Analysis for the Patient Population}

There were two groups of patients in this study, those treated prone with hip fix and those treated supine without immobilization. In both cases the data was in the reference frame rotated $45^{\circ}$ about superior-inferior axis. As will be shown, there was no statistically significant difference between the two groups as per the analysis presented in section 5.3.2.5 and hence for most of this work the results are for the entire patient population.

\subsubsection{Global Systematic Error and Global Random Uncertainty}

Global Geometric error in this study is quantified as the displacement of the prostate (surrogated by fiducial markers) in the BEV from its planned position. The global systematic error and global random uncertainty are further defined as the mean and standard deviation of the global geometric errors for all measured fractions for all patients. The global systematic errors and global random uncertainties in the three observed directions (SI, LO and RO) are summarized in table 5.1. The binned $(<2.5 \mathrm{~mm}$, $2-5 \mathrm{~mm}, 5-10 \mathrm{~mm},>10 \mathrm{~mm}$ ) frequency distribution of the global geometric error from the 257 fractions is also shown in table 5.1. Figure 5.4 is a scatter plot of the global geometric error in an oblique frame. The mean and standard deviation of the global geometric error are measured to be $2.3 \pm 3.8 \mathrm{~mm},-0.3 \pm 4.3 \mathrm{~mm}$, and $1.5 \pm 5.3 \mathrm{~mm}$ in the LO, RO and SI directions, respectively. The largest measured difference between planned and observed prostate position in the 257 fractions was $24.7 \mathrm{~mm}, 18.2 \mathrm{~mm}$ and $28.0 \mathrm{~mm}$ 
in LO, RO and SI directions respectively. The frequency of inter-fraction error being greater than $5 \mathrm{~mm}$ was $25 \%, 27 \%$ and $30 \%$ in LO, RO and SI, respectively while approximately $10 \%$ of all measurements showed an global geometric error of greater than $10 \mathrm{~mm}$, most of which occurred in super-inferior direction.

Table 5.1 Summary of the global geometric errors in three observed directions results of 257 fractions

\begin{tabular}{|c|c|c|c|c|c||}
\hline Directions & Mean \pm Sd $(\mathrm{mm})$ & $\begin{array}{c}<2.5 \mathrm{~mm} \\
(\%)\end{array}$ & $\begin{array}{c}<5 \mathrm{~mm} \\
(\%)\end{array}$ & $\begin{array}{c}<10 \mathrm{~mm} \\
(\%)\end{array}$ & $\begin{array}{c}>10 \mathrm{~mm} \\
(\%)\end{array}$ \\
\hline LO & $-2.3 \pm 3.8(-6.1 \sim 12.1)$ & 45.5 & 27.4 & 25.6 & 1.5 \\
\hline RO & $-0.3 \pm 4.3(-10.1 \sim 14.7)$ & 37.0 & 38.0 & 24.0 & 1.0 \\
\hline SI & $1.5 \pm 5.3(-15.8 \sim 12.2)$ & 32.2 & 38.1 & 20.1 & 9.7 \\
\hline
\end{tabular}

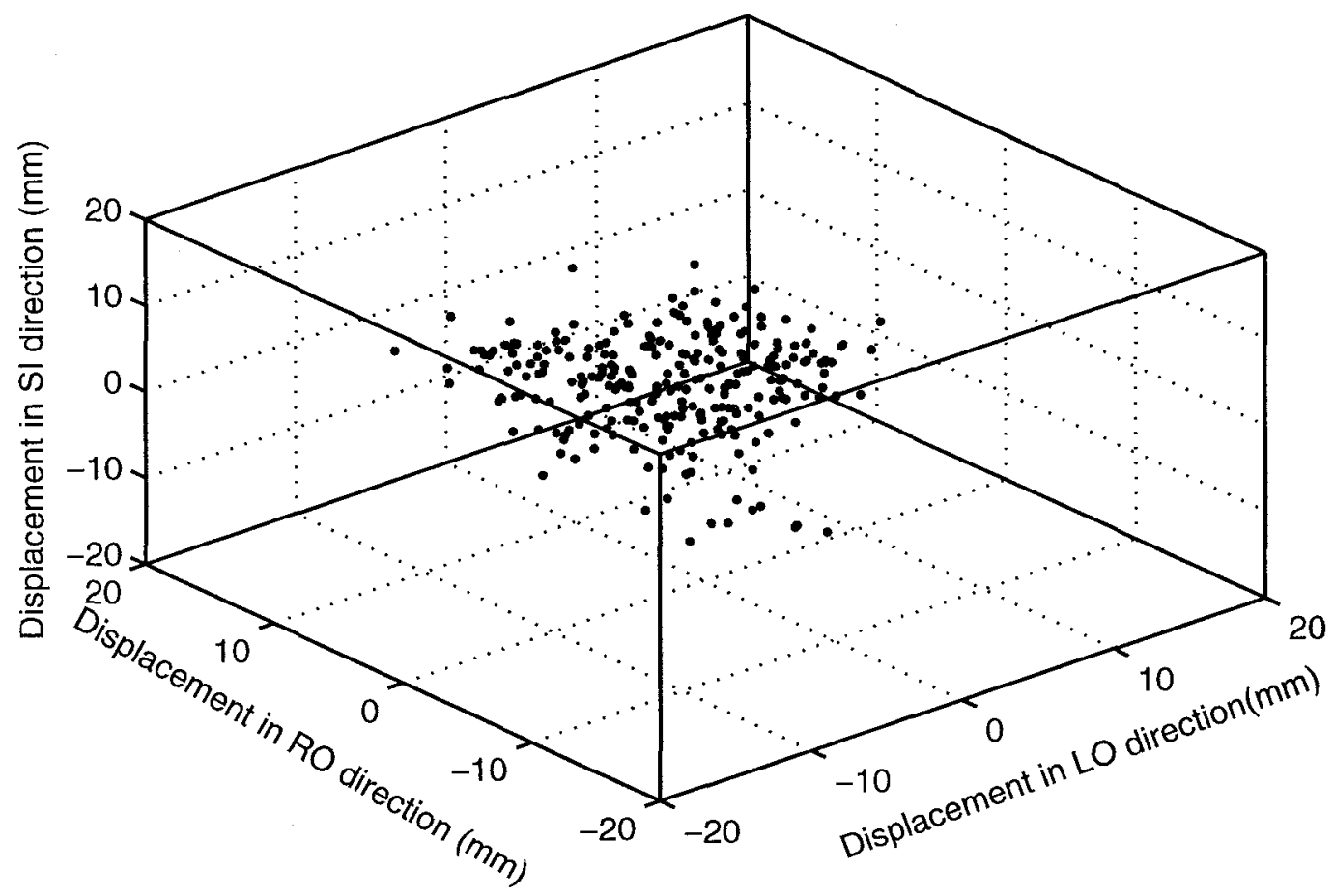

Figure 5.4 Scatter plot of the total daily displacement 


\subsubsection{Treatment Preparation and Treatment Delivery Uncertainties}

The mean and standard deviation, $\overline{\Delta r} \pm \sigma\left(\varsigma^{t}\right)$ of the global geometric error for each of the 21 patients in the three directions (LO, RO, SI) are plotted in Figure 5.5(a) to (c). Note that the mean of the global geometric error is also referred to as the individual systematic error. According to our previous definition, the treatment preparation uncertainty $\Sigma$ is calculated to be the standard deviation of the individual systemic errors $\overline{\Delta r}$ while the treatment delivery uncertainty $\sigma$ is calculated to be the quadratic average $\sigma\left(\xi^{t}\right)$ of all patients. Table 5.2 summarizes treatment preparation and treatment delivery uncertainties in the LO, RO, SI directions for the entire patient group.

Table 5.2 Treatment preparation $(\Sigma)$ and treatment delivery $(\sigma)$ uncertainties

\begin{tabular}{||c|c|c|c||}
\hline & RO & SI & LO \\
\hline$\Sigma$ & $3.2 \mathrm{~mm}$ & $4.5 \mathrm{~mm}$ & $3.5 \mathrm{~mm}$ \\
\hline$\sigma$ & $2.7 \mathrm{~mm}$ & $3.2 \mathrm{~mm}$ & $2.4 \mathrm{~mm}$ \\
\hline
\end{tabular}

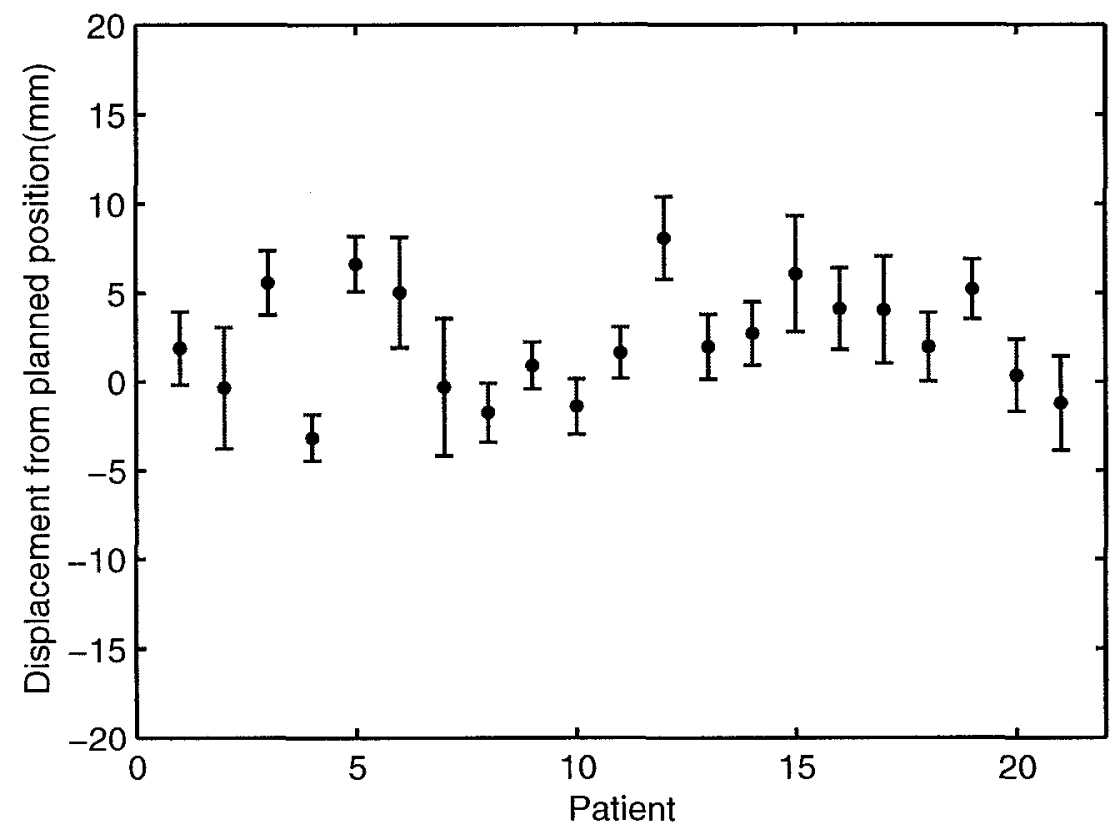

Figure 5.5(a) individual systematic errors in LO direction 


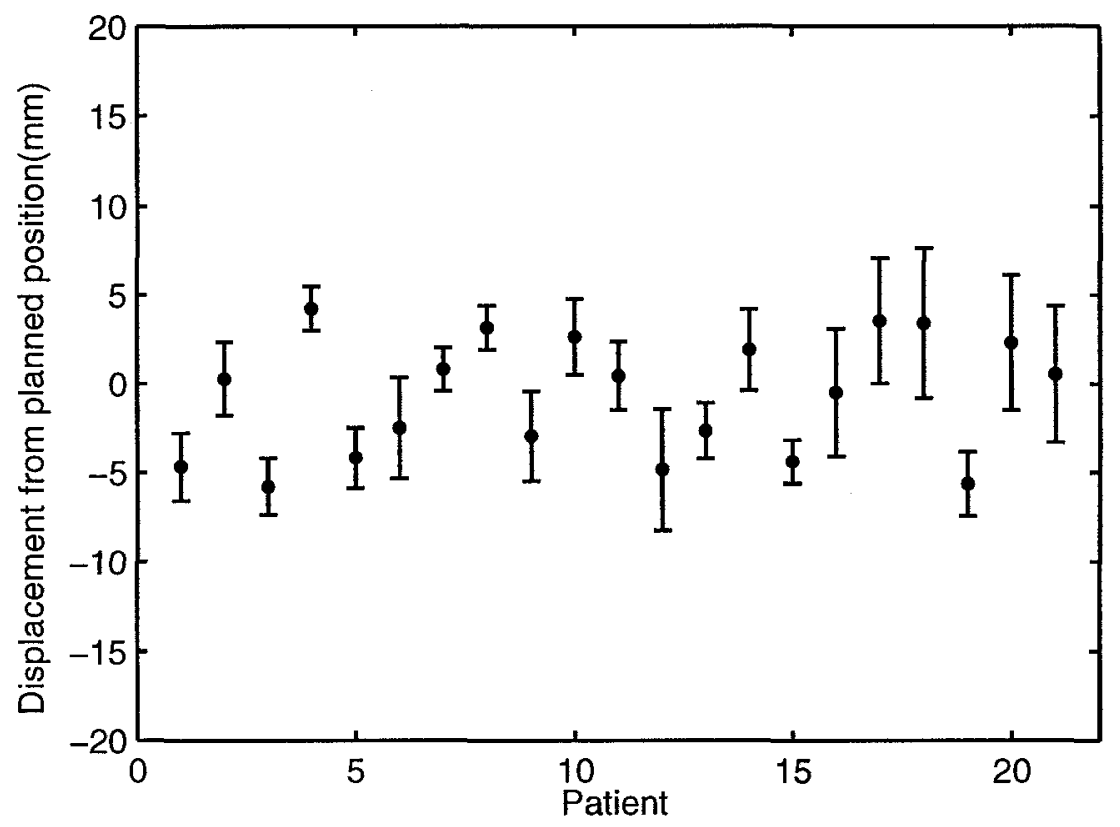

Figure 5.5 (b) individual systematic errors in RO direction

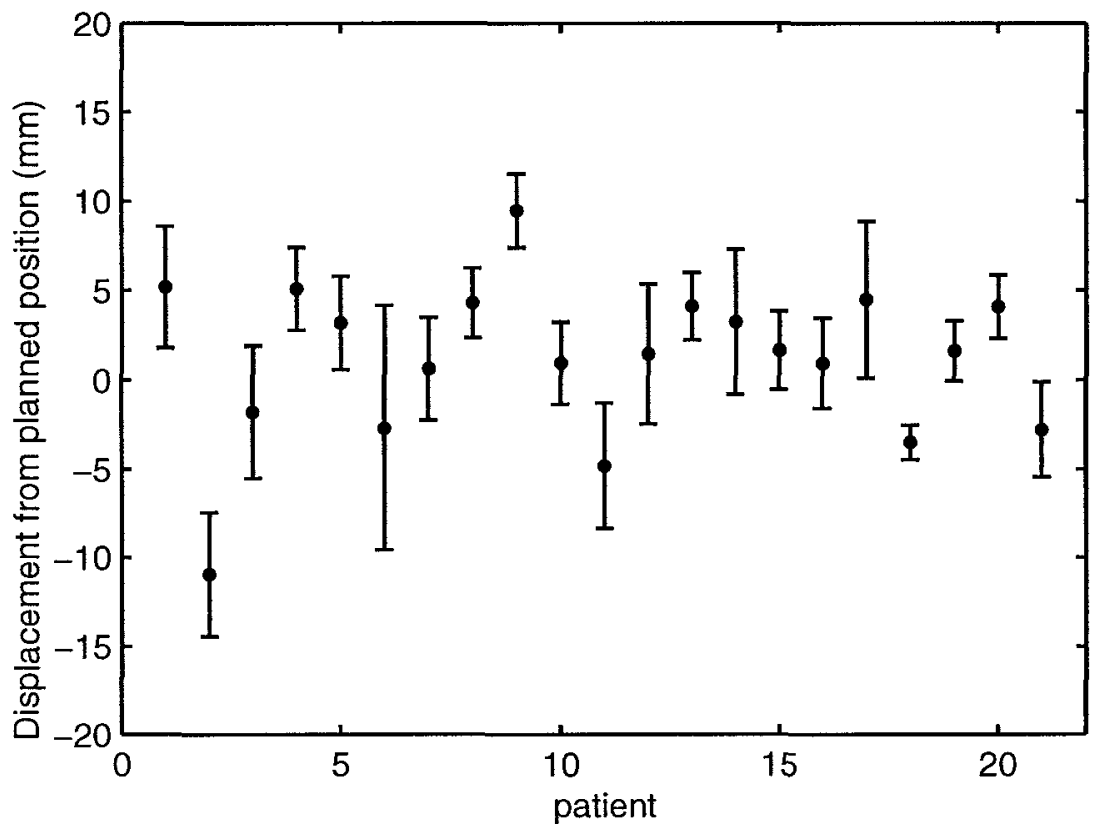

Figure 5.5(c) individual systematic errors in SI direction 


\subsubsection{Intra-Fraction Organ Motion}

In this work we define the difference in the seed COM between two opposed fields as the intra-fraction organ motion. Figure 5.6 is a plot of the means and standard deviations of the intra-fraction organ motion for all patients. As shown in the figure, for most patients the mean intra-fraction error is less than $1 \mathrm{~mm}$ along all axes, but in one patient, (\# 15) we see a systematic shift of $1.6 \mathrm{~mm}$ in the RO direction. Table 5.3 lists means and standard deviations of the intra-fraction organ motion for all fractions in LO, RO and SI directions, respectively. The binned frequency distribution $(<1 \mathrm{~mm}, 1-2 \mathrm{~mm}, 2-3 \mathrm{~mm}$, $>3 \mathrm{~mm}$ ) of the intra-fraction organ motion is also listed in Table 5.3. An intra-fraction organ motion of greater than $1 \mathrm{~mm}$ occurred with a frequency of $16 \%, 20 \%$, and $23 \%$ in LO, RO and SI directions respectively, with corresponding maximum intra-fraction organ motions of $-2.2 \sim 3.5 \mathrm{~mm},-2.5 \sim 3.3 \mathrm{~mm}$ and $-2.6 \sim 3.3 \mathrm{~mm}$.

Table 5.3 Intra-fraction organ motion: results of 257 fractions

\begin{tabular}{||c|c|c|c|c|c||}
\hline Directions & Mean士 sd (mm) & $\begin{array}{c}<1 \mathrm{~mm} \\
(\%)\end{array}$ & $\begin{array}{c}<2 \mathrm{~mm} \\
(\%)\end{array}$ & $\begin{array}{c}<3 \mathrm{~mm} \\
(\%)\end{array}$ & $\begin{array}{c}>3 \mathrm{~mm} \\
(\%)\end{array}$ \\
\hline LO & $0.0 \pm 0.7(-2.2 \sim 3.5)$ & 84.0 & 12.0 & 2.0 & 2.0 \\
\hline RO & $-0.3 \pm 0.8(-2.5 \sim 3.3)$ & 80.0 & 14.0 & 3.8 & 2.2 \\
\hline SI & $-0.2 \pm 1.0(-2.6 \sim 3.3)$ & 77.0 & 18.0 & 4.0 & 1.0 \\
\hline
\end{tabular}

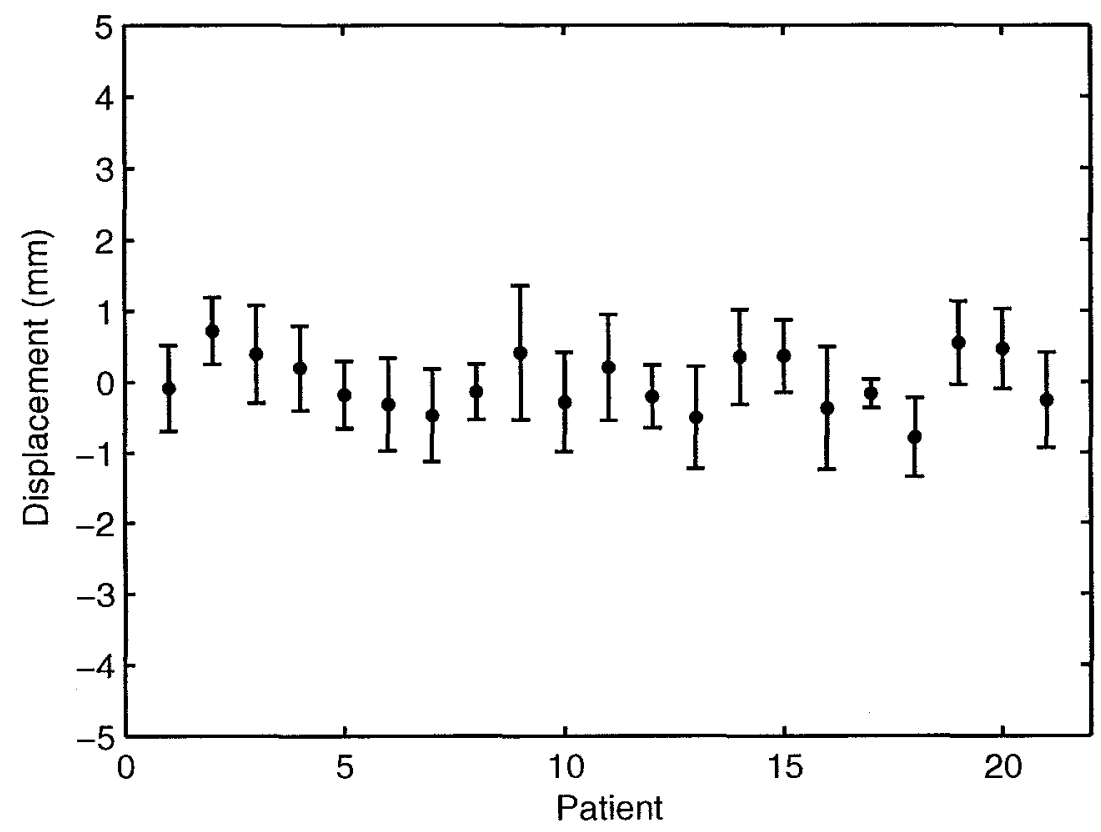

Figure 5.6(a) Intra-fraction organ motions in LO direction 


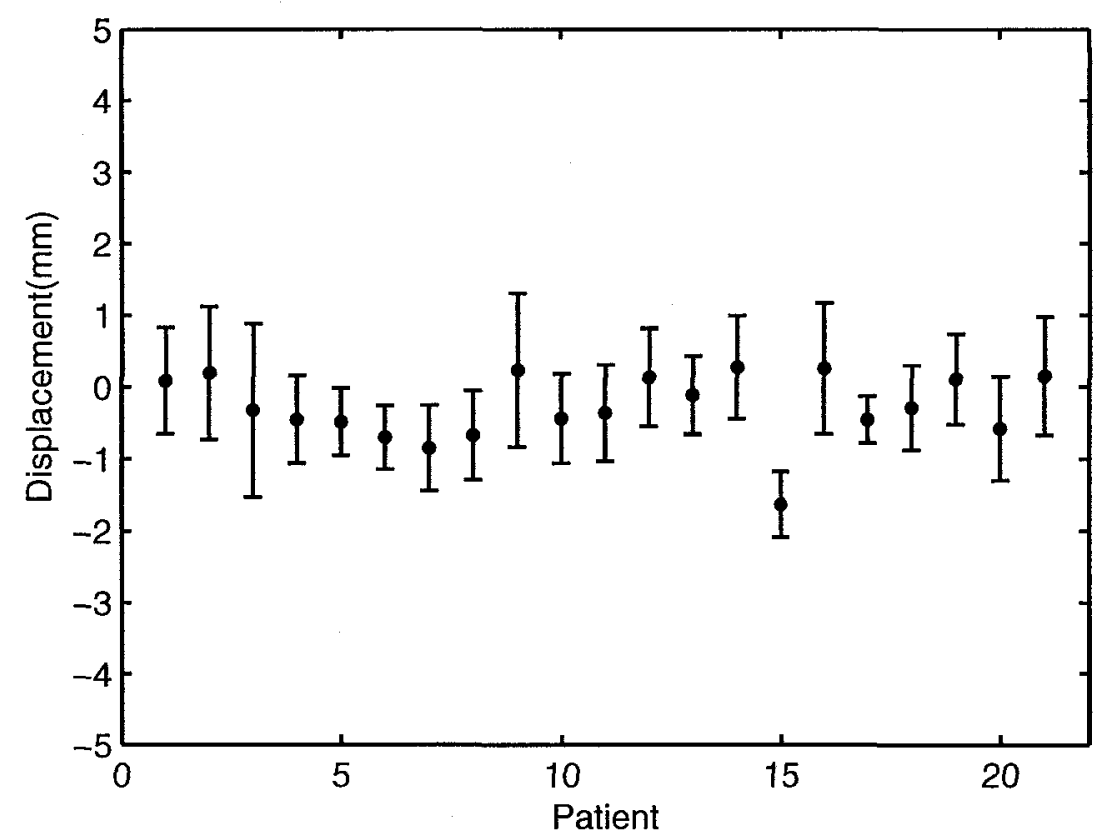

Figure 5.6(b) Intra-fraction organ motions in RO direction

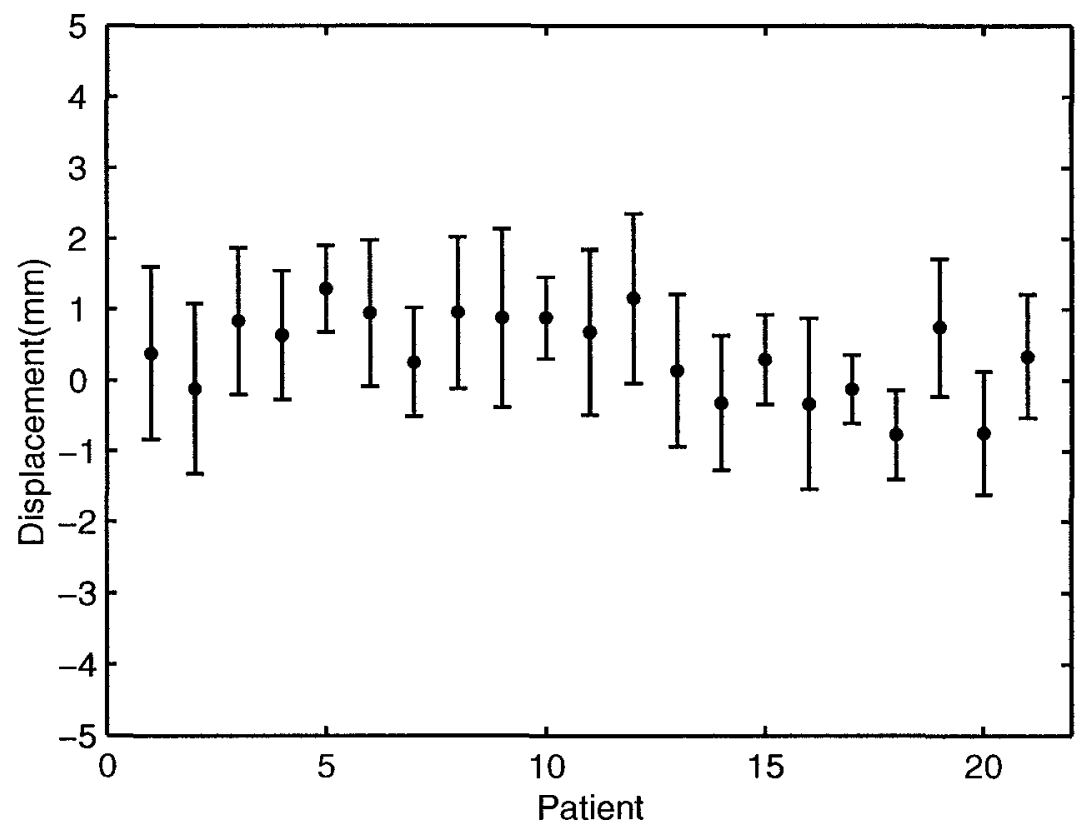

Figure 5.6(c) Intra-fraction organ motions in SI direction 


\subsubsection{Inter-Fraction Organ Motion \& Inter-Fraction Positioning Uncertainties}

Table 5.4 summarizes the inter-fraction positioning uncertainties in LO, RO and SI directions, respectively. The inter-fraction organ motion was decoupled from the target positioning error by subtracting the patient positioning error. The standard deviation of the inter-fraction organ motion in LO, RO and SI directions for the entire patient group are calculated and the results are given in table 5.4.

Table 5.4 Uncertainties in inter-fraction organ motion $\sigma_{o m}$ and patient positioning uncertainty $\left(\sigma_{p e}\right)$

\begin{tabular}{||c|c|c|c|}
\hline Directions & LO & RO & SI \\
\hline$\sigma_{o m}(\mathrm{~mm})$ & 2.1 & 2.6 & 2.8 \\
\hline$\sigma_{p e}(\mathrm{~mm})$ & 1.9 & 2.0 & 2.1 \\
\hline
\end{tabular}

\subsubsection{Examination of the Supine \& Prone Subpopulations}

We have measured inter-fraction error for 73 treatment fractions delivered to 7 supine patients and 184 fractions delivered to 14 prone patients. The mean and standard deviations of global geometric error in LO, RO and SI directions for the prone population were $1.9 \pm 3.9 \mathrm{~mm},-1.3 \pm 3.8 \mathrm{~mm}$ and $1.5 \pm 5.8 \mathrm{~mm}$, respectively while the mean and standard deviations for supine patients were $3.4 \pm 3.0 \mathrm{~mm}, 2.0 \pm 4.4 \mathrm{~mm}$ and $1.5 \pm 3.5$ $\mathrm{mm}$. In both groups the mean of global geometric error is different from zero in all three directions, but none of the differences are statistically significant from zero (student' test $p>0.05$ ). No statistically significant difference in individual systematic error was found between supine and prone group $(p>0.05)$ in any of the three directions ( $F$ test). Similarly, no significant differences were found in target positioning error between the supine and prone groups $(p>0.05)$.

Figures 5.7(a) and (b) are scatter plots of the individual systematic error $\overline{\Delta r}$ in the LO and RO directions for the supine and prone groups respectively. Additionally, the target 
positioning errors $\zeta^{t}$ are also shown on the same graph for comparison. We found that there is a strong correlation in the individual systematic error between the LO and RO directions for the prone group (Pearson's coefficient $=0.86$ ) and the supine group (Pearson's coefficient $=0.70$ ). The correlation coefficients for target positioning error between the $\mathrm{LO}$ and RO directions are 0.46 and -0.17 for prone and supine respectively. Following a series of $\mathrm{R}$ tests, it was found that there is significant correlation between the systematic error and target positioning error between the LO and RO directions $(p<0.05)$ for the prone subgroup. However, the correlation of systematic error and target positioning error between LO and RO directions are not statistically significant for the supine subgroup. The strong correlation in geometric error between the LO and RO directions suggests there is a principle axis along which most of geometric errors occur. We further assume that both the geometric errors in the LO and RO directions are completely projected from this principle axis. We found that this principle axis coincided

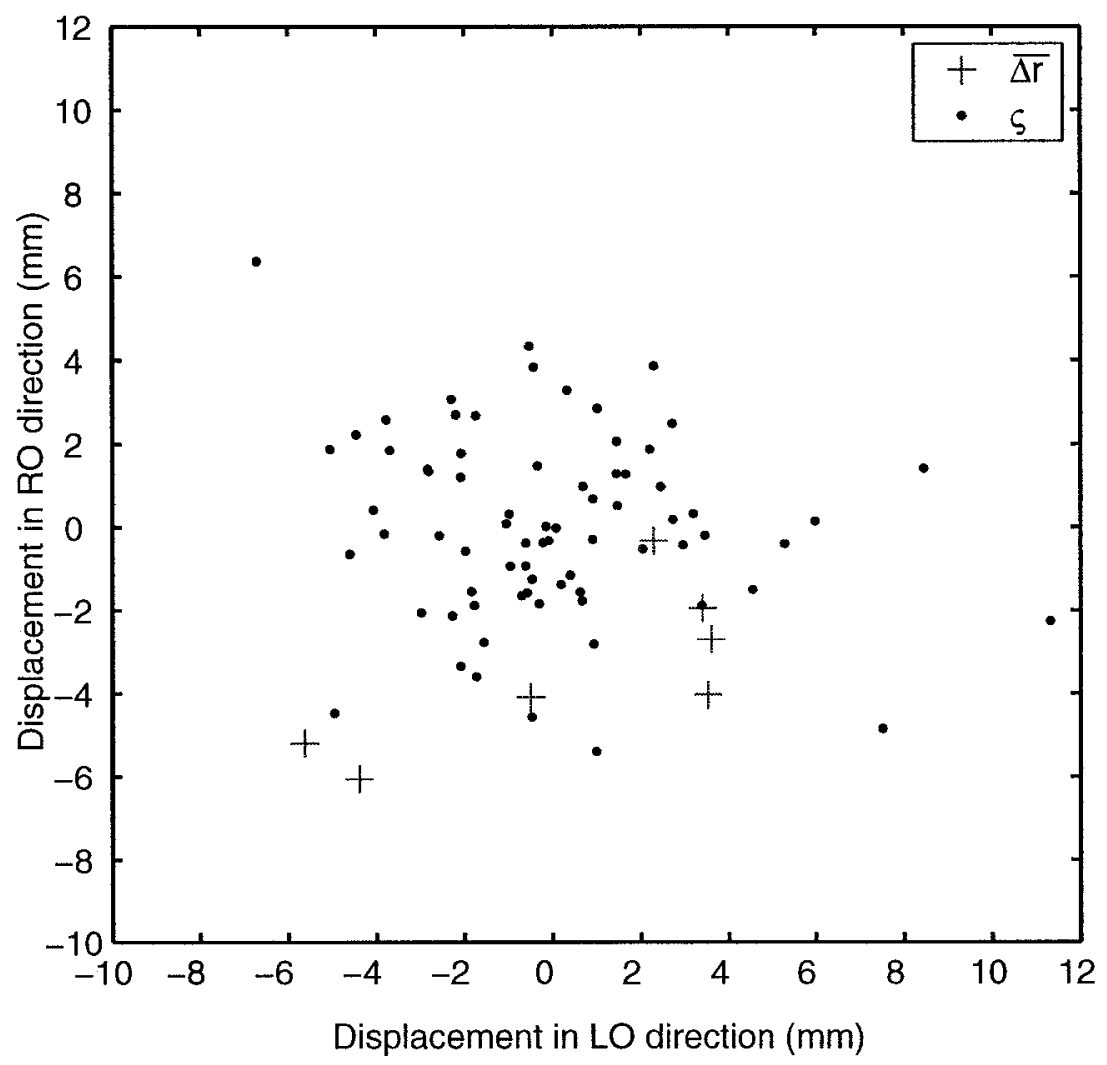

Figure 5.7(a) Scatter plot for supine patients 


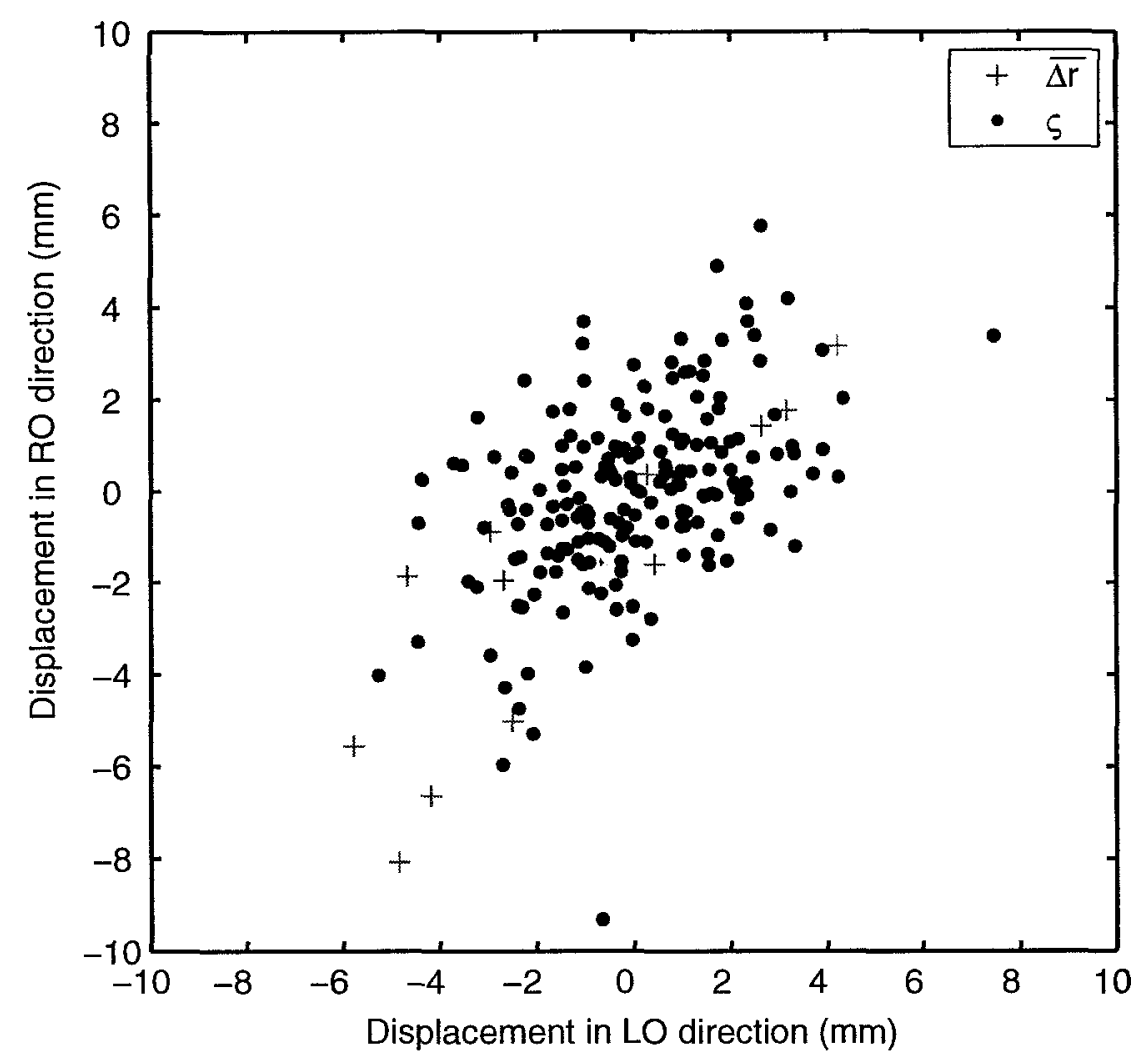

Figure 5.7(b) Scatter plot for prone patients

approximately with the patient's Anterior-Posterior axis. This indirectly confirms that the $\mathrm{AP}$ axis is the dominant direction of the geometric uncertainties for treatment with the prone position as reported by many investigators ${ }^{[41,73]}$

\subsubsection{Uncertainty in Collimator Calibration Error}

We have collected the MLC/Jaw quality assurance (QA) data acquired over the period from March 1999 to April 2004 for the linear accelerator used in this study. The systematic and standard deviation of the solid Jaw and MLC positioning error are summarized in Table 5.5. In addition, we have also collected the QA data for the mechanical isocentre of collimator, couch and gantry obtained over the four year period from 2002 to 2005 . The standard deviations of the isocentre and the range are also summarized in Table 5.5 . 
Table 5.5 MLC/Collimator calibration uncertainties

\begin{tabular}{|c|c|c|c|c|c|}
\hline Errors & Isocentre & Head(Y1) & Foot(Y2) & Left(X1) & Right(X2) \\
\hline Mean (mm) & - & -0.4 & -0.4 & 0.2 & -0.7 \\
\hline SD (mm) & 0.6 & 0.8 & 0.6 & 0.7 & 0.8 \\
\hline Min (mm) & -2.5 & -3.2 & -2.1 & -1.9 & -3.5 \\
\hline Max (mm) & 2.5 & 3.2 & 2.4 & 2.5 & 2.0 \\
\hline
\end{tabular}

This historical data indicates that the accuracy of the isocentre is within $0.6 \mathrm{~mm}$, much less than its tolerance of $1 \mathrm{~mm}$. The uncertainty of the beam-shaping device is within 0.8 $\mathrm{mm}$ for all four edges, which is also much better than its tolerance of $1.5 \mathrm{~mm}$.

\subsection{Discussion}

\subsubsection{Oblique and Conventional Reference Frame}

In this study, most of the results are reported in an oblique frame, rotated $45^{\circ}$ clockwise about the caudal-cranial (or superior - inferior) patient axis, also referred to as the $\mathrm{Y}$ axis in the conventional IEC frame. The use of the oblique frame is based on the following considerations:

Firstly, global geometric errors were measured using images acquired from oblique fields such as LAO, LPO, RPO and RAO, and can be directly reported in the oblique frame $\left(x^{\prime}, y^{\prime}, z^{\prime}\right)$. Oblique images acquired within a fraction were temporally separated by 30 to 120 seconds and intra-fraction prostate (organ) and patient motion could occur during this period. Triangulation of these data to project into the conventional frame could result in errors in the estimation of treatment uncertainties in the AP and LR planes.

Secondly, four of the six treatment portals used in TOHRCC prostate treatment protocol are oblique, and it important to know the motion in the BEV in order to determine the appropriate border between the CTV and PTV. Note that most of the data reported in the literature are for the conventional frame providing for example $\sigma_{L R}$ in LR axis and $\sigma_{A P}$ in AP axis independently. This is adequate for determining boundaries in the AP 
$\mathrm{BEV}$ or Lateral BEV, but not in the oblique planes as discussed below. Although the range of treatment uncertainties in the non-conventional frame can be estimated to be of the order of $\sqrt{\sigma_{L R}^{2}+\sigma_{A P}^{2}}$ from uncertainty data in conventional frame, the exact magnitude of which is unknown due to the unknown principal axis of the geometric errors. As in the example shown in Figure 5.7 (a), the uncertainty of target positioning error $\varsigma^{t}$ along AP axis can be approximately estimated as $\sqrt{\sigma_{L O}^{2}+\sigma_{R O}^{2}}$ for prone (principal axis coincided with the AP axis), but $\sqrt{\left(\sigma_{L O}^{2}+\sigma_{R O}^{2}\right) / 2}$ for supine (no principal axis was presented). The treatment uncertainty reported in the oblique rather than the conventional axes (AP and LR) is therefore a necessary complement for geometric uncertainties in our six-beam 3DCRT, particularly for treatment margin definition.

\subsubsection{Assumptions and Limitations of Methods used in This Work}

The TOHRCC prostate treatment protocol consists of six beams, two opposed sets of oblique pairs and two lateral opposed fields. Each of the opposed fields are planned to be identical in shape when flipped $180^{\circ}$ about their Superior-Inferior axis. This feature is used to determine the inter-field (i.e. intra-fraction) motion of the prostate. It relies on the fiducial seeds as surrogate for the prostate and assumes that the COM of the surrogates lies at the linac isocentre. Based on historical data and on treatment planning requirements, it is safe to assume that the true seed COM lies within a sphere of $50 \mathrm{~mm}$ diameter, centered on the true isocentre. In this case the maximum error in determining the prostate position because of errors in magnification is less than $0.4 \mathrm{~mm}$ and is ignored.

The method described herein requires a reliable reference for motion, which we have chosen to be the beam isocentre. The beam isocentre, or axis of rotation of the collimator, is not absolutely known in each image and must be determined. We have developed and introduced here a method that uses a combination of interleaf leakage and cross correlation with the MLC leaf template to determine the intercept of the beam isocentre 
on an image. Our phantom experiments have shown that we can determine the isocentre to within $0.7 \mathrm{~mm}$ and $0.6 \mathrm{~mm}$ in horizontal and vertical directions respectively.

In this study, three fiducial seeds were implanted as a surrogate for prostate and we assumed constant inter-seed position during the entire treatment course.. The assumption of zero seed migration during the treatment course is another limitation of this study.

\subsubsection{Summary of Independent Geometric Uncertainties}

In this work, we define the global geometric error as the vector sum of the individual systematic error, inter-\& intra-fraction organ motion, patient positioning error, and machine / collimator calibration errors. These individual uncertainties were measured in an oblique frame and are summarized in table 5.6. Note that target definition errors and post delineation deformation of the target are also sources of geometric uncertainty in prostate cancer radiotherapy. Neither of these are considered as part of the global geometric error, but are listed in table 5.6. The target definition error was determined from the VHP study reported later in this thesis. It is defined as the random uncertainty in the distance in the plane of projection between delineated and true prostate volumes. The target deformation error, not measured in this work is extracted from Dehard ${ }^{[73]}$. The methods for determining the uncertainties are described in detail in chapter 3.

Table 5.6 Summary of the independent uncertainties

\begin{tabular}{||c|c|c|c|}
\hline Sources & LO & RO & SI \\
\hline Delineation Uncertainty $\left(\sigma_{T D}\right)$ & & \multicolumn{3}{|c|}{$2.9 \mathrm{~mm}$} \\
\hline Preparation Uncertainty $(\Sigma)$ & $3.5 \mathrm{~mm}$ & $3.2 \mathrm{~mm}$ & $4.5 \mathrm{~mm}$ \\
\hline Intra-fraction organ motion $\left(\sigma_{\text {int } r a, O M}\right)$ & $0.7 \mathrm{~mm}$ & $0.8 \mathrm{~mm}$ & $1.0 \mathrm{~mm}$ \\
\hline Inter-fraction organ motion $\left(\sigma_{\text {int } e r, O M}\right)$ & $2.1 \mathrm{~mm}$ & $2.6 \mathrm{~mm}$ & $2.8 \mathrm{~mm}$ \\
\hline Positioning uncertainty $\left(\sigma_{P E}\right)$ & $1.9 \mathrm{~mm}$ & $2.0 \mathrm{~mm}$ & $2.1 \mathrm{~mm}$ \\
\hline Method uncertainty & & $1.0 \mathrm{~mm}$ \\
\hline Collimator calibration uncertainty & & $1.0 \mathrm{~mm}$ \\
\hline Target deformation & & $1.0 \mathrm{~mm}$ \\
\hline Overall uncertainty & $5.3 \mathrm{~mm}$ & $5.4 \mathrm{~mm}$ & $6.3 \mathrm{~mm}$ \\
\hline
\end{tabular}


From the 1028 EPID images, acquired during 257 patient treatments, we have extracted the beam isocentre, field edges, the fiducial markers and a surrogate bony landmark. These data were analyzed to determine the geometric uncertainties in prostate cancer EBRT. The global random uncertainty or overall uncertainty is the square root sum of all uncertainties in each direction for the entire patient population and was measured to be 3.8, 4.3 and $5.3 \mathrm{~mm}$ in $\mathrm{LO}, \mathrm{RO}$ and SI directions respectively.

\subsubsection{Analysis of Systematic Error and Target Positioning Error}

As discussed in chapter 2, systematic error can modify the dose distribution to the CTV while target positioning error only blurs the dose distribution. It is worthwhile to examine the cause and nature of these two different types of errors. $\operatorname{Yan}^{[36]}$ found that the individual systematic error $\overline{\Delta r}$ and target positioning error $\zeta^{t}$ follow similar distributions if both are measured over a large number of fractions and in a large patient population. This can be explained with reference to Figure 5.8 Assume that the prostate position $x$ is normally distributed with a global mean of zero and standard deviation $\sigma$. The planned position for the prostate $x_{0}$, is determined from a single snapshot of the prostate, which is

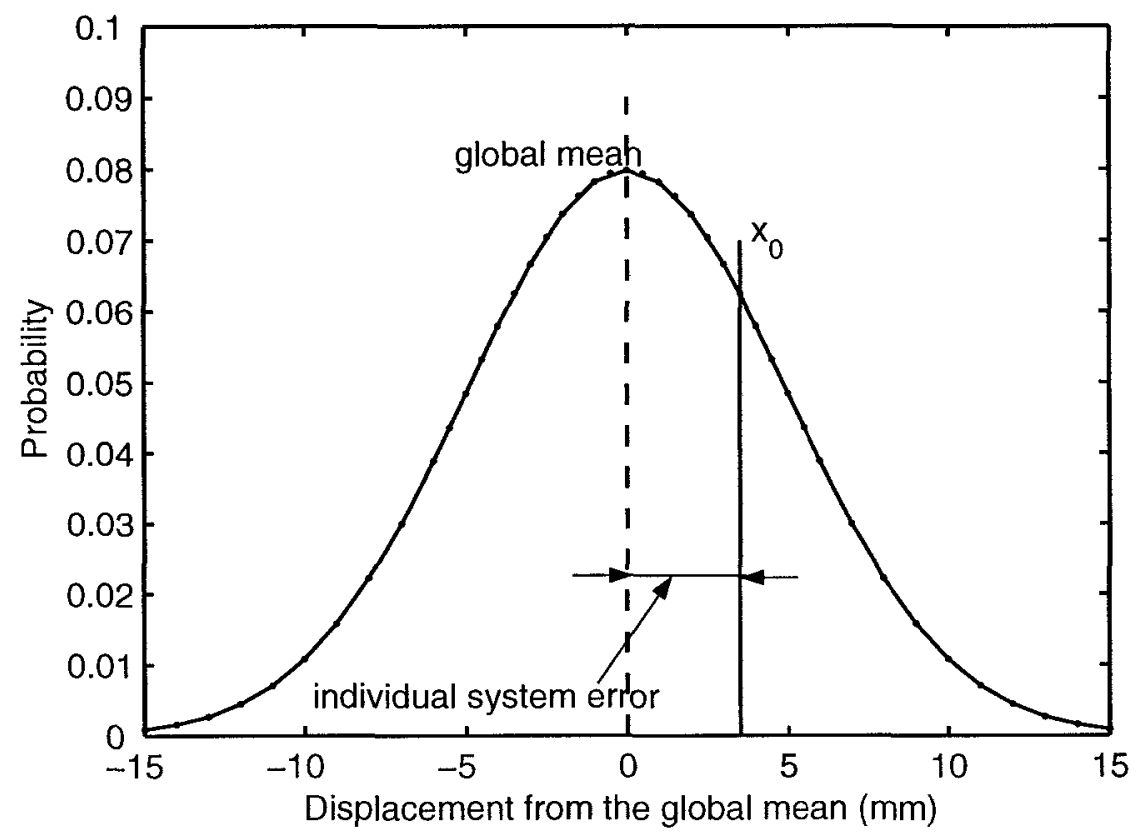

Figure 5.8 Systematic error versus random error 
in theory sampled from the distribution $\sigma$. Following $N_{f}$ fractions of treatment, the individual systematic error $\overline{\Delta r}$ for a patient can be estimated as $\hat{x}_{0}\left(N_{f}\right)-x_{0}$ while the uncertainty in target positioning from $\hat{x}_{0}\left(N_{f}\right)$ (mean measured position) can be estimated to be $\hat{\sigma}\left(N_{f}\right)$. Note $\hat{x}_{0}\left(N_{f}\right)$ is the mean observed prostate position during the $N_{f}$ fractions of radiation delivery. It can be shown that $\hat{x}_{0}\left(N_{f}\right) \cong 0$ and $\hat{\sigma}\left(N_{f}\right)=\sigma$ as $N_{f}$ approaches infinite.

The treatment preparation uncertainty can be described by $\widehat{\Sigma}=\sqrt{\hat{\sigma}^{2}\left(N_{f}\right) / N_{f}+\hat{\sigma}^{2}\left(N_{p}\right)}$ while the uncertainty in delivery can be estimated as $\hat{\sigma}=\hat{\sigma}\left(N_{p}, N_{f}\right)$ for a population of $N_{p}$ patients. Thus $\widehat{\Sigma}=\hat{\sigma} \cong \sigma$ if both $N_{f}$ and $N_{p}$ are sufficiently large. Note that in this study $N_{f}$ ranges from 8 to 20 fractions and the patient population, $N_{p}$ is 21 . Because the sample size is not significantly large $(>100)$, there is more statistical error in our assessment of treatment preparation uncertainty.

\subsubsection{Requirements and Usefulness of Routine Portal Check Films}

In conventional radiotherapy, verification films (or check films) are often taken to "assure" the physician that the patient or target is well positioned for treatment. For patient setup verification, the displacement is determined by comparing certain anatomical structures in a check image to the reference image. If the physician determines that the setup error is greater than some value, usually $5 \mathrm{~mm}$, the patient's reference setup is changed introducing a systematic change in subsequent treatment fractions. At TOHRCC, six verification films are usually taken over the course of a 38 fraction treatment. This sampling frequency does not have a theoretical basis, but has "evolved" from physician preference and the impact of this sampling on patient outcome (or dose distribution) has not been assessed locally. Subsequent to the acquisition of the data in this study an analysis of global geometric error can provide guidance for what 
would constitute a reasonable frequency (or sample size) for check films in this patient population.

Table 5.1 lists the frequency distribution of the global geometric error in the three directions. It is seen that the global geometric error (geometric sum of all errors) is greater than $5 \mathrm{~mm}$ in $60 \%$ of all measured fractions (154 out of 257 fractions) implying that based on the criteria presently used at TOHRCC the patient would be shifted approximately every other fraction with a prerequisite that global geometric errors are known for all fractions in a treatment course.

We further assume that the individual systematic error $\overline{\Delta r}$ could be corrected prior to the radiation delivery. This leaves only target positioning error $\varsigma^{t}$ contributing to the global geometric error. By applying the same shift criteria, patients needed to be shifted in 32 out of 257 observed fractions (12\%). This suggests that on average 5 shifts are required for a typical 38-fraction treatment for prostate cancer. This finding also implies that individual systematic error is the largest cause of the patient shift.

Furthermore, assuming that the global geometric error is only caused by positioning error $\omega$, it was found that patients would need a position shift in 10 of 243 fractions by applying the $5 \mathrm{~mm}$ shift criteria. This suggests that only 2 shifts are required for a typical 38 fraction treatment. However, under Nyquist-Shannon sampling theorem ${ }^{[121]}$, it would require at least 4,10 and 38 pretreatment portal images to detect a global geometric error of more than $5 \mathrm{~mm}$ due to all sources of errors, target positioning error, and patient positioning error, respectively.

Frequency analysis of the global geometric errors for different sources of geometric errors demonstrates that the utilization of check or seed films in conventional radiotherapy is not an effective strategy to reduce the treatment deviations. This is because check films are utilized only for correcting patient positioning error, but we have demonstrated that patient positioning error alone contributes to approximately $7 \sim 8 \%$ of 
the patient shifts. It is found that individual systematic error actually is the dominant source of the patient shift, however, is ignored in the check film approach.

\subsubsection{Strategies to Reduce Individual Systematic Error}

As illustrated in Figure 5.8, individual systematic error is due to an inaccurate knowledge of the mean target position at the time the reference position (i.e. planning position) is determined. This error could be effectively reduced if the planned target position $\boldsymbol{x}_{0}$ was replaced with a mean prostate position obtained through multiple simulations acquired during the planning process. Figure 5.9 is a plot of the percent systematic error removed as a function of the number of independent image acquisitions for simulation. This figure demonstrates that $40 \%$ or $48 \%$ of systematic error would be removed if three or four simulations were performed as part of the planning process before radiation delivery. In our case, uncertainties in the preparation stage along the three observed directions could be reduced to $1.7,1.8$ and $2.3 \mathrm{~mm}$ if four simulations are performed for each patient. Multiple simulations can effectively reduce individual systematic error, but it is very expensive in terms of simulation time and resource costs. Yan ${ }^{[67]}$ suggested that the mean target position can be estimated by averaging the target position over the first several fractions. Once the mean prostate position is estimated based on images from the first few fractions, the isocentre can be shifted to reflect the updated information and this will reduce the systematic error for the remaining fractions. As a consequence, the remaining fractions are optimized. For conventional 3D conformal radiotherapy, this strategy can not only improve dose conformity, but also reduce the normal tissue toxicity at a minimum cost. 


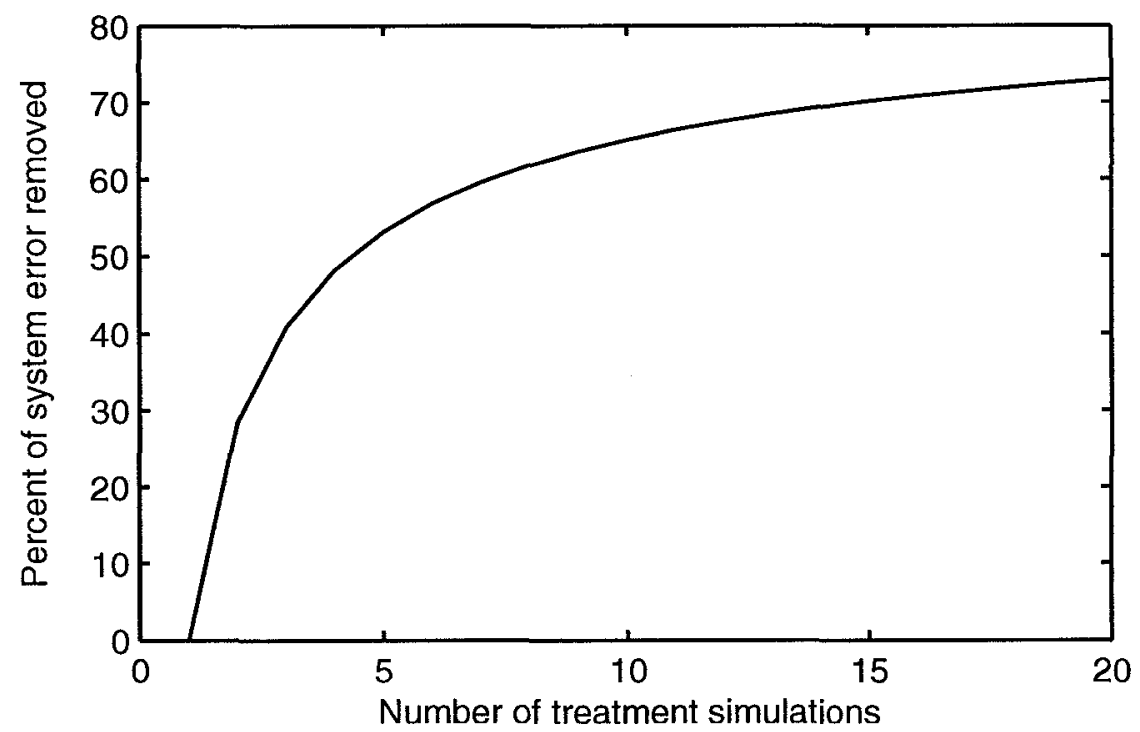

Figure 5.9 Percent of systematic error removed as a function of number of simulations

\subsubsection{Uncertainties in Preparation and Delivery Stages}

There have been many studies ${ }^{[36,51,65,73]}$ examining patient positioning error and interfraction organ motion using different imaging techniques. Yan ${ }^{[36]}$ has investigated prostate motion and daily positioning error by using an average of 18 consecutive CT scans as well as approximately 100 portal images per subject for 30 patients. He found the systemic component of the positioning error to be $2.9,1.9$ and $1.8 \mathrm{~mm}$ in the AP, SI and LR directions respectively. Similarly he found the random component to be $2.2,1.8$ and $2.2 \mathrm{~mm}$ in AP, SI and LR directions respectively. Their study also found that the inter-fraction organ motion (uncertainty) was $3.6 \mathrm{~mm}$ and $2.5 \mathrm{~mm}$ in the AP and SI directions during the pretreatment planning phase and 2.6 and $2.0 \mathrm{~mm}$ during treatment delivery. Van Herk ${ }^{[68]}$ reported that the total overall treatment preparation uncertainty was $4.5,4.1$ and $3.2 \mathrm{~mm}$ in $\mathrm{AP}$, SI and LR respectively, while the overall delivery uncertainty was $3.2,2.5$ and $2.2 \mathrm{~mm}$. These values resulted from several studies conducted at the Netherlands Cancer institute using EPID and film. ${ }^{[51,68]}$ Similarly, another group $^{[73]}$ also from Netherlands, reported preparation uncertainties of 4.8, 3.5 and $3.3 \mathrm{~mm}$ and delivery error of $3.2,2.2$ and $2.1 \mathrm{~mm}$ in AP, SI and LR respectively. However, none of these studies has reported results in the oblique directions, the only 
common axis being the SI direction. In our work the treatment preparation uncertainty was found to be $3.2,3.5$ and 4.5 in the LO, RO, and SI directions respectively. Interestingly the largest error in our study was in the SI direction $(4.5 \mathrm{~mm})$, while both Van Herk $(4.5 \mathrm{~mm})$ and Dehnad $(4.8 \mathrm{~mm})$ report the maximum error in the AP direction. This clearly has implications for determining the most appropriate PTV boundaries in the $\mathrm{BEV}$, depending on whether the beam is a conventional lateral/AP or an oblique field as in the TOHRCC protocol. It is unclear why our data differs from other reports, but it is likely that much of the error is process related, specifically linked to patient immobilization, patient teaching, and staff training.

\subsubsection{Intra-Fraction Organ Motion}

There is limited literature regarding intra-fraction organ motion. Vigneault ${ }^{[77]}$ investigated intra-fraction prostate motion in two patients with fiducial markers using EPIDs and reported no intra-fraction motion. Padhani ${ }^{[138]}$ measured prostate motion in 55 patients with cine MRI every 10s during a period of 7 mins. In 9 of the patients he reports an intra-fraction motion greater than $5 \mathrm{~mm}$ in AP direction. In a fluoroscopic study, Malone ${ }^{[69]}$ measured the maximum prostate displacement over a period of 20 s for 40 patients, reporting motions of $2.9 \pm 1.7 \mathrm{~mm}$ and $1.6 \pm 1.1 \mathrm{~mm}$ in SI and AP directions, respectively. Recently, Aubry ${ }^{[74]}$, using consecutive triangulation of implanted gold seeds in two orthogonal images, reported $0.8 \mathrm{~mm}, 1.4$ and $1.0 \mathrm{~mm}$ mean intra-fraction motion in LR, AP and SI directions for 282 observed fractions. Huang ${ }^{[44]}$ measured the intrafraction prostate motion using pre and post treatment ultrasound imaging and reported distributions of intra-fraction motions of $0.4 \mathrm{~mm}, 1.3 \mathrm{~mm}$ and $1.0 \mathrm{~mm}$ in the LR, AP and SI directions.

The intra-fraction organ motions in this study were found to be normally distributed with a mean near zero and a standard deviation of $0.7 \mathrm{~mm}, 0.8 \mathrm{~mm}$ and $1.0 \mathrm{~mm}$ in LO, RO and SI directions over 257 fractions. The intra-fraction organ motion in the SI direction $(0.1 \pm 1.0 \mathrm{~mm})$ is consistent with that reported by Aubry $(0.4 \pm 1.0 \mathrm{~mm})$ and by Huang 
$(0.1 \pm 1.0 \mathrm{~mm})$. The greatest error observed in our 257 fractions was smaller than that observed by either Huang ${ }^{[44]}$ or Aubry. ${ }^{[74]}$

It is difficult to compare oblique measurements with the AP and LR measurements reported by others, particularly because most of the literature reports these motions separately as excursions along a single axis. In order to translate into an oblique plane the trajectory of motion is required. Several studies ${ }^{[44,74]}$ suggest that the intra-fraction prostate motion is greatest in the anterior-posterior direction while others report the greatest intra fraction motion in the superior-inferior direction ${ }^{[69]}$. In order to estimate maximum intra-fraction prostate motion in the AP direction, we assume that the intrafraction prostate motion in the LO and RO directions are direct projections from the motion in the patient's AP axis, giving an AP motion of $\sqrt{\sigma_{L O}^{2}+\sigma_{R O}^{2}}$. By this means, the maximum AP intra-fraction motion in this study is estimated to be $1.1 \mathrm{~mm}$ which is very close to $1.3 \mathrm{~mm}$ reported by Huang and $1.4 \mathrm{~mm}$ reported by Aubry. ${ }^{[7]}$

\subsubsection{Implications for Determining the PTV}

We have investigated and reported the global geometric error and the intra-fraction prostate motion based on electronic portal images taken during 257 treatment fractions to 21 patients. The prostate position was determined based on fudical markers and the patient position was determined based on the extraction of bony landmarks from within the image. The global geometric error is the vector sum of all treatment preparation (simulation, planning) and delivery (setup positioning error, inter and intra-fraction prostate movement within the pelvis) as well as linac geometric errors. In our study the data were analysed offline and were not used to correct treatment position, hence the global geometric error represents the maximum treatment error one could expect in our practice. Similarly, the intra-fraction organ motion represents the lower limit of the treatment error we could achieve if global geometric error was corrected by advanced IGRT techniques ${ }^{[43]}$ since most image guided techniques are based on pre-delivery images. 
Based on ICRU 50/62, a treatment margin to the CTV is required to account for the overall geometric error. Margin optimization is essential for minimizing normal tissue doses. Several margin recipes incorporating overall treatment errors and based on physical, dosimetric and biological criteria have been reported ${ }^{[50,100-102]}$. Currently treatment margin optimization for oblique beams is restricted by the lack of corresponding geometric uncertainty information. The geometric uncertainty obtained in this study was directly measured from four oblique portal images and therefore can be utilized to determine treatment margins for the corresponding oblique portals. A margin recipe designed to ensure $95 \%$ of prescribed dose for $90 \%$ of patients was suggested by Van Herk ${ }^{[103]}$ and is given as: $d_{t m}=2.5 \Sigma_{T}+0.7 \sigma_{T}$, where $\Sigma_{T}$ and $\sigma_{T}$ are the uncertainties in the total preparation (systematic) and delivery (random) process for a particular beam direction. Using the data from table 5.2, estimation of the margin using the method of Van Herk yields a total margin of 9.6, 10.6 and $13.4 \mathrm{~mm}$ in LO, RO and SI directions, respectively. The minimum margin in SI direction $(13.4 \mathrm{~mm})$ obtained from this study is very close to the $13.5 \mathrm{~mm}$ suggested by Van Herk ${ }^{[103]}$. However, our data suggests that the margins required for the oblique fields are 9.6 and $10.6 \mathrm{~mm}$ (RO, LO) are approximately $3 \mathrm{~mm}$ less than that suggested by Van Herk for lateral field (i.e. ant and post) margins.

It might be inappropriate to directly compare recommended margins among the different institutions because the treatment techniques, particularly immobilization, pretreatment protocols and treatment protocols can be significantly different. For instance, in addition to a variety of immobilization devices, some institutions ensure full bladder ${ }^{[90]}$ while others request that patients void just prior to treatment ${ }^{[41]}$. Similarly some institutions manage a patients diet to control rectal filling. However, the data obtained in this study is useful for examining the most appropriate margin for use at TOHRCC as long as the treatment preparation and delivery process remains unchanged. In the TOHRCC treatment protocol for low or intermediate risk prostate cancer, a uniform margin of 10 $\mathrm{mm}$ is added to the CTV and a treatment plan is developed to encompass this volume by the $95 \%$ isodose. This margin is invariant in the transverse plane for all directions, except that it is reduced to $5 \mathrm{~mm}$ posteriorly (towards the rectum) for the final portion (boost) of 
the patients treatment. Superiorly a margin of $15 \mathrm{~mm}$ is used for the initial and is reduced to $10 \mathrm{~mm}$ for the boost. Similarly the inferior margin is $20 \mathrm{~mm}$ for the initial and is reduced to $15 \mathrm{~mm}$ for the boost. Analysis of our data, using the method of Van Herk ${ }^{[103]}$ found that the current margins of $(10 \mathrm{~mm}+5 \mathrm{~mm}$ penumbra from $50 \%$ to $95 \%$ isodose line) in transverse plane for initial treatment is larger than required for oblique fields $(9.6 \sim 10.6 \mathrm{~mm})$. Our data also suggests that current margin of $10 \mathrm{~mm}$ in the inferior direction for the boost treatment is less than required $(13.4 \mathrm{~mm})$. We have also found that the frequency with which the prostate moves superiorly by more than $10 \mathrm{~mm}$ from the planned position is 13 out of 257 fractions (see Table 5.1), suggesting that this margin might also be reexamined by the treatment program.

It is now becoming common to treat prostate cancer using image-guided techniques such as onboard imaging, Cone beam $\mathrm{CT}^{[97]}$ or . Tomotherapy. ${ }^{[109]}$ In these techniques, the global geometric error can be addressed within the resolution of the imaging modality, but in general image guidance cannot address intra-fraction patient and organ motion. The question now arises, what margin is required for these techniques?

Statistically the inclusion of the intra-fraction target motion as part of the global geometric error adds an extra margin of $0.3 \mathrm{~mm}$ in transverse plane and $0.4 \mathrm{~mm}$ in superinferior direction for 3D conformal therapy. However, if advanced IGRT techniques could eliminate the global geometric error then the statistical contribution of intrafraction target motion in the margin recipe of Van Herk becomes significant. In this case a margin of $2.3 \mathrm{~mm}, 2.6 \mathrm{~mm}$ and $3.3 \mathrm{~mm}$ would be required to address intra-fraction target motion in LO, RO and SI directions, respectively. This margin represents the minimum requirement, assuming that the image guidance was perfect and eliminated all pretreatment and setup errors as well as inter fraction tumour motion. However, some of the geometric errors can not be corrected by using IGRT techniques, these include intra-fraction organ motion $(1 \mathrm{~mm})$, target deformation $(1 \mathrm{~mm})$ during a treatment fraction, method uncertainty $(1 \mathrm{~mm})$ of IGRT and target delineation uncertainty $(2 \sim 3.5 \mathrm{~mm})$. By combining the remaining uncertainty in all factors together, a minimum margin of 6.1 
$\mathrm{mm}$ is required for any IGRT techniques. The uncertainty in prostate target delineation will be examined in chapter 5 .

\subsubsection{Supine versus Prone Treatment Position}

Prostate cancer patients are treated either prone or supine and the method of choice varies from centre to centre. At TOHRCC prostate patients who are medically fit are treated prone in a commercial immobilization device known as the Hip-Fix. Others who find it difficult to lie in this position are treated supine..$^{[139]}$ In this study we have found that there is no significant statistical difference in the various geometric uncertainties $(p=0.05)$ between supine treatment with minimal immobilization and prone treatment using the hip-fix immobilization device. A similar finding was reported by Bayley. ${ }^{[40]}$ It should be noted that many of the radiation therapists have anecdotally reported their doubts regarding the utility of the hip-fix technique, but the lack of documentation and supportive data made change difficult.

Our study suggests that there are two disadvantages in using the Hip-Fix with immobilization. The first is from a patient perspective. Prone positioning with the HipFix technique is not comfortable for the patient. It is also inefficient in that the time required for prone patient setup with the hip-fix is known to be 2-3 minutes longer than that in supine position. At TOHRCC, a radiotherapy linac can treat approximately 30 prone hip fix patients in a standard 8 hour working day. It would save 60 90 minutes if all patients are treated supine, and as suggested by our data without any reduction in treatment quality. Considering that Ontario suffers from long waiting lists for cancer treatment, changing from prone to supine treatments will not only save the cost in HipFix device ( $\$ 300$ per patient), but also should reduce the waiting time. 


\subsection{Conclusions}

We have investigated the geometric uncertainty associated with EBRT of prostate cancer using electronic portal images of 257 fractions to 21 patients at the Ottawa Hospital Regional Cancer Centre.

The global geometric error is the vector sum of all treatment preparation (simulation, planning) and delivery (positioning error, intra-fraction patient motion, inter and intra fraction prostate movement within the pelvis) as well as linac geometric errors. The intrafraction organ motion is the target motion within a fraction. The global geometric error is a measure of the maximum error one could expect. Similarly intra-fraction organ motion represents the lower limit of the treatment error that can be achieved assuming that there is no real-time measurement and correction for intra fraction motion. Based on our measurement, the global random geometric uncertainty is estimated to be within $5.3 \mathrm{~mm}$ for all three directions of observation. If both target delineation and target deformation uncertainties are included, the overall geometric uncertainty in prostate cancer treatment would be of the order of $6.3 \mathrm{~mm}$. The intra-fraction organ motion was found to be within $1 \mathrm{~mm}$ for all observed direction. Therefore this uncertainty is small with respect to the overall geometric uncertainty and the effect of intra-fraction prostate motion can be ignored for $3 \mathrm{D}$ conformal radiotherapy, however, is significant when developing a treatment margin for IGRT.

The global geometric error was also broken down between treatment preparation and treatment delivery uncertainties. Treatment preparation uncertainty describes the distribution of the geometric error that accumulates prior to treatment delivery while treatment delivery uncertainty describes the geometric uncertainty during radiation delivery. Although both treatment preparation and treatment delivery uncertainties come from the same distribution, we have found that the treatment preparation uncertainty is generally larger than treatment delivery uncertainty. This phenomenon was mainly due to their sample size difference. It is also important to note that in routine practice 
pretreatment uncertainty translates into systematic error while delivery error is usually random.

Based on our analysis, the utilization of the check or seed film to reduce treatment deviations is questionable because it can only correct random delivery error such as setup error or internal target motion, but not the dominant systematic error. The utilization of check film can only correct $7 \%$ of all significant deviations.

The systematic error in prostate cancer radiotherapy is due to an inaccurate knowledge of the mean target position prior to dose delivery. This can be effectively reduced if more treatment simulations are performed. Alternatively, we can also estimate the mean target position over first several treatment fractions, after obtaining more accurate mean target position, the isocentre should be updated.

The result of this study can also be used to guide the selection of the treatment margin. Putting our geometric data in the margin model of van Herk, we found that current margin $(10 \mathrm{~mm})$ in the inferior direction for boost treatment is too tight, but that it is reasonable in the transverse plane.

By comparing a variety of geometric uncertainties between supine and prone with HipFix immobilization, we found there is no significant difference between the two techniques. However, supine treatment is superior to prone with hip-fix in terms of patient comfort and resource utilization. 


\section{CHAPTER 6 Modeling the Biological Effect of Geometric Errors in EBRT}

\subsection{Overview}

This chapter describes the effect of geometric uncertainty on local tumour control for prostate cancer EBRT. The Linear-Quadratic cell survival model and associated parameters are discussed in section 6.2. Several radiobiological concepts including tumour control probability, biological effective dose, equivalent uniform dose and normal tissue complication probability are also introduced. The effect of geometric errors is simulated for combinations of two different size margins and three different treatment techniques. The treatment techniques include 3DCRT without clinical intervention, with positioning error correction and with full geometric error correction. Section 6.3 summarizes the results from the simulations.

\subsection{Introduction}

Cancer is a biological system consisting of many cancerous cells. The aim of radiotherapy is to destroy this system by decreasing the number of viable cancerous cells to as few as possible. In theory, the number of tumour cells which remain after radiation treatment is dependent upon how many cancerous cells there were prior to treatment, upon the response of the cells to radiation and upon the dose delivery patterns. Because geometric uncertainty during radiotherapy affects the dose distribution, it also plays a major role in cell kill. As demonstrated in Chapter 5, there is a large variation in prostate target delineation amongst radiation oncologists. This introduces a large uncertainty in the determination of the initial number of target cells to be killed. Additionally, we have 
shown that the presence of geometric uncertainty causes the delivered dose to be different than the planned dose distribution to the target.

It is difficult to predict individual patient outcomes, but the effects of process changes can be assessed using radiobiological models. ${ }^{[140]}$ Among the many radiobiological models used to describe tumour response ${ }^{[140,141]}$, the linear-quadratic model is the most widely accepted by radiobiologists because it fits the clinical data well and has descriptive utility. In this study, the effect of geometric uncertainties on patient outcome is simulated using the fractionated linear-quadratic model in combination with a Poisson survival model. The geometric uncertainties addressed in this work include patient positioning error, internal target motion and individual systematic error. Three treatment techniques for prostate cancer were simulated. These techniques are 3DCRT without clinical intervention, with positioning error correction and with full geometric error correction.

Treatment strategies developed through radiobiological modeling will depend strongly on the assumptions in the model. The treatment course developed to treat prostate cancer assuming a relatively low $\alpha / \beta$ ratio (e.g $<1.5 \mathrm{~Gy}$ ) is very different than the strategy which would be adopted if we assume a high $\alpha / \beta$ ratio $(>8.5 \mathrm{~Gy})$. This has been discussed extensively in the literature ${ }^{[86,99,142]}$, but as of yet there is still no definitive consensus as the most appropriate value for the $\alpha / \beta$ ratio for prostate cancer. In this study, we emphasize the impact of geometric uncertainty on tumour control and assume a moderate value of 3.1 for the tumour $\alpha / \beta$ ratio, which is very similar to the $\alpha / \beta$ ratio for normal tissues such as rectum. As a result, no therapeutic gain can be obtained from the difference in $\alpha / \beta$ value between rectum and prostate.

Adaptive techniques using image-guided radiotherapy (IGRT) and online target repositioning techniques ${ }^{[97]}$ have been recently developed to reduce normal tissue toxicity. Employing these techniques provides a mechanism for dose escalation to the tumour while maintaining normal tissue complication at an acceptable level. Unfortunately, with the present technology, these techniques are not efficient in their use 
of resources and hence the optimization for prostate cancer using IGRT techniques has not been sufficiently investigated. The simulation introduced here can provide guidance with respect to the use of adaptive techniques for the treatment of prostate cancer.

\subsection{The Linear Quadratic Model}

The Linear-Quadratic (LQ) model ${ }^{[141]}$ assumes that the sensitive sites for radiationinduced cell lethality are located in the nucleus. The principal targets for ionizing radiation-induced chemical transformation are the DNA molecules. A DNA molecule consists of two strands that form a double helix, each strand composed of a series of deoxynucleotides, the sequence of which contains the genetic information required for self-replication and cell division. Thus damage to the DNA of a cell may lead to a loss of genetic coding critical to cellular function and survival. The critical damage is assumed to be a double-strand break (DSB). DSBs in a DNA molecule are capable under certain conditions of being repaired. The repair processes include chemical restitution and enzymatic repair. The linear quadratic model further assumes that two different mechanisms can lead to DNA damage. One is that both strands are broken by one radiation event. Another is that each strand is broken independently and the breaks are close enough in time and space for the molecule to rupture. It is thought that the linear quadratic model provides a description of this process and is written as

$$
s_{f}=\exp \left(-\alpha D-\beta D^{2}\right)
$$

where $s_{f}$ is the surviving fraction after a total dose D (Gy) to a homogeneous tumour. The parameters $\alpha$ and $\beta$ can be interpreted as the lethality induced by damage from a single track (one radiation event) and that from two independent tracks (multiple radiation events) of ionizing particles, respectively.

The linear quadratic model has been modified to account for a variety of factors. These factors include dose rate, fractionation, oxygen, protraction, LET, repair rates and tumour 
growth during treatment. Several of the modified LQ models are described in the next few sections.

\subsubsection{The Linear Quadratic Model with Dose Protraction}

The LQ model with a repair term is given by:

$$
s_{f}=\exp \left(-\left(\alpha D+\beta G D^{2}\right)\right)
$$

where $\mathrm{G}$ is the Lea-Catcheside dose- protraction factor, and is given by Sachs ${ }^{[143]}$ as:

$$
G=\frac{2}{D^{2}} \int d t \dot{D}(t) \mathcal{L}_{-\infty} d t^{\prime} \dot{D}\left(t^{\prime}\right) e^{-\lambda\left(t-t^{\prime}\right)}
$$

where $\dot{D}(t)$ is the absorbed dose rate at time $t$. The biophysical interpretation of the equation is that a potentially lethal lesion, presumably a double strand break, is created at time $t^{\prime}$ and if not repaired, may interact with a second lesion produced at time $t$. The rate of the repair is characterized by a constant $\lambda$, or by the effective half-time for repair $\tau=0.693 / \lambda$. This modified model has been used by several authors ${ }^{[144-146]}$ to investigate the radiobiological effect of dose protraction for prostate cancer treatment with EBRT.

\subsubsection{The Fractioned Linear Quadratic Model}

Treatment fractionation is accepted as the standard delivery model for external beam radiotherapy. In this technique, the total radiation dose is divided into a number of fractions and is delivered to the tumour site daily over a specific period. It has been known for a long time that there is significant improvement in uncomplicated tumour control with fractionated doses of radiotherapy as opposed to a single large dose or a few large fractions. The biological basis for fractionated radiotherapy is that tumour tissues are generally more sensitive to the radiation (less likely to repair) than the surrounded normal tissues. The advantage of this technique is that the total tolerance dose for the 
normal tissues is extended due to the repair of sub-lethal damage between two fractions. Because of the reduced normal tissue toxicity, the dose to the tumour can be escalated and a lower survival fraction of tumour cells can be achieved. This is demonstrated in the Figure 6.1. The theory behind the fractionation technique is described by the so-called "4Rs": Repair, Reoxygenation, Redistribution, and constitution of the shoulder of the survival curve between two fractions. The probability for sublethal repair improves as the number of dose fractions increases, resulting in a sparing effect to the normal tissues. This sparing effect is more noticeable among the late-responding normal tissues as shown in Figure 6.1. The presence of oxygen increases tumor cell radiosensitivity while hypoxia has the opposite effects. During a fractionated treatment session, a process of reoxygenation has been shown to occur. This phenomenon can be explained as follows: well-oxygenated tumor cells are close to the tumour blood supply. These are more radiosensitive and hence are killed off first. When these cells die the distant hypoxic cells have an increased opportunity for oxygen, making them less hypoxic and more radiation sensitive.. Thus the oxygen status within a tumour continually changes between 
fractions and tumour cells are more easily killed. It has also been found that cells have different sensitivity to the radiation in certain portions of the cell cycle. Fractionation provides an opportunity for cells to progress (or redistribute) through the cell cycle, allowing previously radio-resistant cells to enter a more radiation sensitive part of the cell cycle. ${ }^{[140]}$.

The fractionated linear quadratic model can be given by:

$$
s_{f}=\left(e^{-\alpha d-\beta d^{2}}\right)^{n_{f}}=e^{-\alpha D-\beta d D}
$$

where the parameter $d$ is the fraction size and $n_{f}$ is the fraction number. As shown in equation 6.4 , unlike tumour response to a single dose, tumour response to fractionated radiotherapy is not only a function of the total dose $D$, but also the fraction size $d$.

\subsubsection{Biological Effective Dose}

In order to describe the tumour response to radiation while incorporating the effect of fractionation, the concept of Biological Effective Dose (BED) was suggested by Brendsen ${ }^{[147,148]}$ and subsequently defined by Fowler ${ }^{[148]}$ as:

$$
B E D=-\frac{\ln \left(s_{f}\right)}{\alpha}
$$

Applying the LQ model, the BED is then expressed as:

$$
B E D=D\left(1+\frac{D / n_{f}}{\alpha / \beta}\right)=D\left(1+\frac{d}{\alpha / \beta}\right)
$$


The BED is an important concept because it can differentiate between fractionation regimes $(d)$ and tissue sensitivity $(\alpha / \beta)$ to the radiation dose. This concept provides a measure to quantify the biological effect for different fractionation schemes and different tissue sensitivities. Under this concept, the total physical dose becomes meaningless without specification of the fractionation scheme. The weakness of this concept is that a homogeneous dose is assumed to be delivered to a homogeneous tumour. This is clearly not generally valid, in particular for a target with substantial motion and a spectrum of cells with large uncertainties in radiosensitivity such as in prostate cancer.

\subsubsection{Equivalent Uniform Dose (EUD)}

To address inhomogeneous dose distributions in the tumour, Equivalent Uniform Dose (EUD) was suggested by Niemierko ${ }^{[149]}$. The EUD was developed based on two assumptions: the first is that the tumour is composed of a large number of independent colonogens. The second is that random cell killing by radiation is well described by either Poisson statistics or binary response. Thus, two different dose distributions delivered to the target are equivalent if the expected number of surviving cells is equal. The EUD is defined as the dose that can lead to the same survival fraction for a given dose distribution to the irradiated tumour. This is given as

$$
s_{f}(E U D) \equiv s_{f}(D)
$$

where $D$ is any dose distribution over a tumour with $\mathrm{N}_{\mathrm{s}}$ irradiated sub volumes or voxels. If clonogens in each subvolume $v_{i}$ are independent of those in other subvolumes, then:

$$
s_{f}(B E D)=\prod_{i=1}^{i=N_{s}} v_{i} e^{-\alpha D}
$$

where $B E D_{i}$ can be defined by equation 6.5 or 6.6 , 


$$
E U D=-\frac{\ln s_{f}(E U D)}{\alpha}=-\frac{1}{\alpha} \ln \left(\prod_{i=1}^{i=N_{s}} v_{i} e^{-\alpha D_{i}}\right)
$$

The introduction of EUD addresses the weakness of BED. In addition, EUD is more practical for evaluating clinical doses such as defining the effect of an inhomogeneous dose distribution to tumours. EUD also makes it possible to rank treatment plans with only one physical parameter, and has been applied in many clinical studies ${ }^{[142,150]}$ since its introduction. EUD can also explain the effect of dose heterogeneity and fractionation schemes. However this concept is still a physical unit, and cannot solve the problem such as tumour heterogeneity.

\subsubsection{Tumour Control Probability}

Following cancer radiotherapy, tumour control probability (TCP) is often chosen as the biological indicator to predict the treatment outcome. TCP is defined as the probability of killing all cancer cells within a tumour after a certain radiation dose D. In theory, all cancerous cells must be killed to achieve complete local control. However, the stochastic nature of the interaction between radiation and tumour cells makes it inappropriate to use a deterministic quantity such as yes or no to describe the treatment outcome. Assume that all tumour cells have a similar radiosensitivity and the number of surviving cells $N_{s}$ after exposures to total dose $\mathrm{D}$ is given by

$$
N_{s}=N_{0} s_{f}(D)
$$

In order to have a high probability of cure a cancer, the probability of surviving cancerous cells must be very small. The tumour control probability after a specific radiation dose is calculated with a Poisson distribution with the probability of no surviving cancerous cells in the tumour. This is the so-called Poisson tumour control probability, which can be represented as follows: 


$$
T C P=e^{-N_{s}}=e^{-N_{0} s_{f}(D)}
$$

The surviving fraction is usually defined by the linear-quadratic model and $N_{0}$ is the number of initial cancer cells. Using the assumption of independent sub volumes with heterogeneous exposure, the overall probability of tumour control is the product of each probability of killing all cancerous cells in each sub volume $i$ (or voxel). This is the socalled voxel based tumour control probability. The TCP for heterogeneous dose over a tumour target is defined by Webb ${ }^{[81]}$ as follows:

$$
T C P=\prod_{i=1}^{M_{s v}} T C P_{i}
$$

Voxel based TCP can be used to assess the treatment outcome from external beam radiotherapy without assuming either a homogeneous dose or a homogeneous tumour.

\subsubsection{Normal Tissue Complication Probability}

Healthy tissues surrounding a tumour are inevitably irradiated during radiation treatment. When a normal tissue is irradiated it may lose partial or total function fail. Normal tissue complication probability is defined as the probability that a certain percentage of patients have unfavorable reactions in the normal tissue at a certain dose level. Assuming a uniform dose is delivered to a portion of an organ (e.g. rectum or bladder for prostate cancer), the normal tissue complication probability can be given as:

$$
N T C P=\frac{1}{\sqrt{2 \pi}} \int_{-\infty}^{t_{n t}} e^{-\frac{t^{2}}{2}} d t
$$

where $t_{n t}$ is the number of standard deviations that the actual total dose $\mathrm{D}$, is apart from the tolerance dose, $T D_{50}$, which is derived from: 


$$
t_{n t}=\frac{D-T D_{50}}{m_{n t} * T D_{50}}
$$

$\mathrm{TD}_{50}$ is the dose delivered to the entire volume of the normal tissue leading to $50 \%$ complication probability, and $m_{n t}$ is the slope parameter of the dose-response curve. For normal tissues, the relative volume of the organ irradiated to a certain dose is used to predict the complication probability. The dose-volume relationship differs for each organ due to the different tissue architectures. The dose-volume relationship was suggested by Lyman ${ }^{[151]}$ and is described using the parameter $n_{n t}$ in the power law:

$$
T D_{50}=T D_{50}(v)^{n_{n t}}
$$

where $v$ is the volume fraction of an organ receiving the dose $\mathrm{D}$, and $\mathrm{TD}_{50}(v)$ is the tolerance dose for $50 \%$ of the patient population when irradiating a volume fraction. $n_{n t}$ is the fitting parameter accounting for the volume effect. Table 6.1 includes the parameter values of $\mathrm{TD}_{50}, n_{n t}$, and $m_{n t}{ }^{[152]}$ for NTCP calculation for several organs as recommended by Burman. ${ }^{[152]}$

Calculating NTCP of an organ for a heterogeneous dose distribution using the Lyman model was subsequently extended by Kutcher. ${ }^{[153]}$ In his formula, the dose-volume histogram of an organ was applied to calculate the effective volume $v_{\text {eff }}$ :

$$
v_{e f f}=\sum_{i=1}^{\max } \Delta v_{i} \frac{D_{i}}{D_{\max }}
$$

where $\Delta v_{i}$ is the volume fraction receiving a total biological effective dose $\mathrm{D}_{\mathrm{i}}$, and $\mathrm{D}_{\max }$ is the maximum dose to the organ. For a normal tissue or an organ at risk, the calculation of NTCP is seriously affected by both volume and tissue architecture. The concept of a functional subunit $(\mathrm{FSU})^{[140]}$ has been introduced into the NTCP estimation to differentiate the sensitivity of various tissues to radiation. This theory assumes that a human organ is composed of many connected FSUs and each FSU or several of them can 
complete a particular function and as well a certain number of FSUs can complete an organ's function. The NTCP is thus dependent on the types of FSUs. For example, an organ (e.g. lung) is resistant to radiation if the FSUs are paralleled. In contrast, an organ (e.g. spine) is radiosensitive if the FSUs are serially connected. Note that a mixt of serial and paralleled FSUs within an organ makes it difficult to predict the NTCP for an organ.

When applying tumour control probability and normal tissue complication probability in clinical studies, we must be aware of the limitations of the two concepts. These are summarized as follows.

Table 6.1 parameter values for NTCP calculation

\begin{tabular}{||c|c|c|c|}
\hline ORGAN & $\mathrm{TD}_{50}(\mathrm{~Gy})$ & $n_{n t}$ & $m_{n t}$ \\
\hline Rectum & 80 & 0.12 & 0.15 \\
\hline Bladder & 80 & 0.50 & 0.11 \\
\hline Kidney & 28 & 0.70 & 0.10 \\
\hline
\end{tabular}

First, the dose distribution over a tumour (CTV) is usually non-homogeneous owing to radiation delivery fluctuations, patient positioning error and patient/organ motion. Meanwhile, the clonogen cell distribution within a tumour is also spatially variant. Additionally the radiosensitivity (tissue/tumour sensitivity, crucial biological response of hypoxia, intra-tumor sensitivity variation, cell growth, and cellular repair mechanisms) also changes with the delivery patterns. Both TCP and NTCP are strongly affected by these factors.

Secondly, TCP and NTCP are statistical concepts that describe the possibility of local tumour control or normal tissue complication for a population with the same type of tumours and with similar doses. It is inappropriate to apply this concept to predict the outcome for an individual patient. 
Based on the local tumour control probability and the normal tissue complication probability, the concept of the uncomplicated tumour control probability (UTCP) is defined as:

$$
U T C P=T C P(1-N T C P)
$$

Figure 6.2 is a plot of TCP, NTCP, and UTCP as a function of the total radiation dose. As shown in the figure one can achieve a better outcome only if one can separate the TCP and NTCP curves along the dose axis.

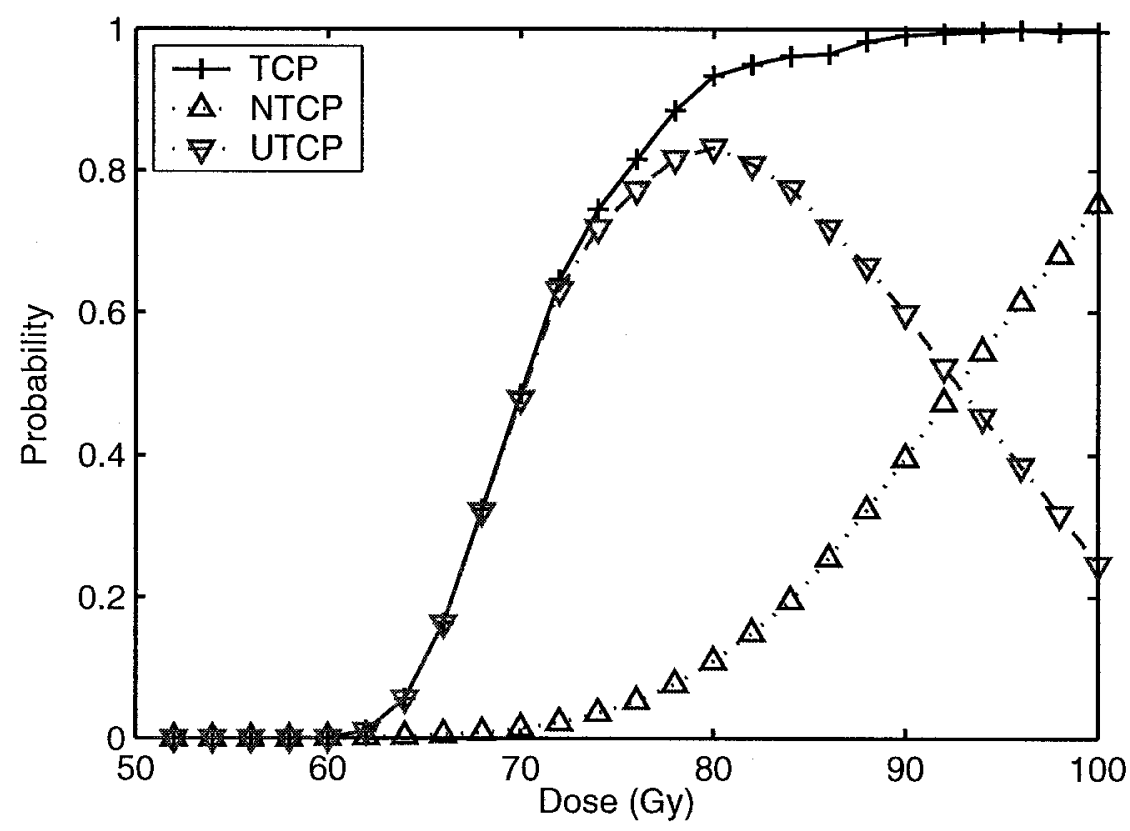

Figure 6.2 Relationship among TCP, NTCP and UTCP 


\subsection{The Effect of Geometric Errors on Local Tumour Control}

Modeling the effect of geometric uncertainties on local control requires both treatment error data and tumour-radiation response data. In this simulation, the treatment error data were obtained from the clinical measurements performed at the TOHRCC and reported earlier in chapter 5. The radiobiological parameters were harvested from the literature. Both sources of data are described in next few sections.

\subsubsection{Geometric Uncertainties}

According to Van Herk ${ }^{[68,103]}$, the global geometric error associated with prostate cancer EBRT can be described by the geometric uncertainties in the preparation or in the delivery stages. Patient positioning uncertainty, inter-and intra-fraction organ motion along with preparation uncertainty are taken into account in this simulation. These data are summarized in table 6.2 and are based on image analysis work reported in chapter 4 for each of four oblique images acquired from 257 fractions delivered to 21 patients. Please note that the uncertainty data along the AP and LR axes were triangulated from the measurements in patient oblique frame ( $L O, R O)$, without consideration for the effect of intra-fraction organ motion.

Table 6.2 Summary of geometric uncertainties in overall treatment process

\begin{tabular}{|c|c|c|c||}
\hline Sources & LR & AP & SI \\
\hline Preparation uncertainty & $2.6 \mathrm{~mm}$ & $4.2 \mathrm{~mm}$ & $4.5 \mathrm{~mm}$ \\
\hline Intra-fraction motion & $0.7 \mathrm{~mm}$ & $1.2 \mathrm{~mm}$ & $1.0 \mathrm{~mm}$ \\
\hline Inter-fraction motion & $1.4 \mathrm{~mm}$ & $2.9 \mathrm{~mm}$ & $2.8 \mathrm{~mm}$ \\
\hline Positioning uncertainty & $1.7 \mathrm{~mm}$ & $2.1 \mathrm{~mm}$ & $2.1 \mathrm{~mm}$ \\
\hline
\end{tabular}




\subsubsection{Radiobiological Parameters}

The radiobiological parameters of interest in the linear-quadratic model include $N_{0}$, the number of initial cancer cells, $\alpha$ and $\alpha / \beta$ ratio.

Several investigators have published different values of parameters for $\alpha$ and $\alpha / \beta$ for prostate cancer cells. The parameter $\alpha$ has been estimated by Nahum ${ }^{[154]}$ as $0.26 \pm 0.07$ $\mathrm{Gy}^{-1}$ and the $\alpha / \beta$ was then determined to be $8.4 \mathrm{~Gy}$ assuming $5 \times 10^{6}$ initial clonogenic cells in the CTV. Wang ${ }^{[144,155]}$ and Brenner ${ }^{[145,146]}$ have also suggested significantly different values for $\alpha$ and $\alpha / \beta$ and the number of initial clonogens. These are all compared in Table 6.3.

Because it has a huge impact on the treatment strategy for prostate cancer radiotherapy, the issue of a very low $\alpha / \beta$ ratio has been intensively debated in the literature. For many years, the value of the $\alpha / \beta$ ratio has been assumed to be $10 \mathrm{~Gy}$ for a typical early responding tumour, but this has recently been challenged. Based on the analysis of two sets of tumour control data, one using EBRT data and the other with permanent seed implants, the sensitivity to changes in fractionation of prostate cancer was extracted. From this Brenner ${ }^{[145]}$ estimated a value of 1.5 for the $\alpha / \beta$ ratio (1999) and later in 2002 estimated an even lower value of $1.2^{[146]}$. His results suggest that the prostate cancer is much more sensitive to fractionation than most tumours $(\alpha / \beta>8 \mathrm{~Gy})$, and in fact his estimated value is also lower than for most late responding normal tissues $(\alpha / \beta \sim 4 \mathrm{~Gy})$.

Table 6.3 Parameter sets used in the calculations of tumour control probability for external beam radiotherapy

\begin{tabular}{|c|c|c|c||}
\hline \multirow{2}{*}{} & \multicolumn{3}{|c|}{ PARAMETER VALUES } \\
\cline { 2 - 4 } & Nahum $^{[154]}$ & Brenner $^{[145]}$ & Wang $^{[155]}$ \\
\hline$\alpha$ & $0.26 \mathrm{~Gy}^{-1}$ & 0.026 & 0.15 \\
\hline$\alpha / \beta$ & $8.4 \mathrm{~Gy}$ & 1.2 & 3.1 \\
\hline$N_{0}$ & $5 \times 10^{6}$ & 138 & $3 \times 10^{6}$ \\
\hline
\end{tabular}


By using an alternative analysis incorporating the effects of hypoxia and inter-patient heterogeneity, Nahum et $\mathrm{al}^{[154]}$ found that a set of parameter values $\alpha=0.26 G y^{-1}$, $\alpha / \beta=8.4 G y$ could also fit the clinical data used by Brenner. Wang ${ }^{[155]}$ suggested a third set of parameter values for the calculation of tumour control probability. In his analysis, the effects of dose rate, sublethal damage repair, and clonogenic proliferation were all considered. He estimated $0.15 \mathrm{~Gy}^{-1}, 3.1 \mathrm{~Gy}$ and $10^{6} \sim 10^{7}$ for $\alpha, \alpha / \beta$ and the number of initial tumour cells $N_{0}$, respectively.

A low $\alpha / \beta$ value for prostate carcinoma implies that the use of a large number of fractions to reduce late responding normal tissue complications might be unsuccessful because the prostate is more sensitive to the change in fraction number than lateresponding normal tissues. This leads to the suggestion for hypofractionation schemes to increase the local tumour control without increasing the risk in late responding normal tissue complication. ${ }^{[146,148]}$ According to Brenner, ${ }^{[156]}$ the benefits of hypofractionation for prostate cancers are summarized as follows:

- Hypofractionation has comparable tumour control probability and late responding normal tissue complication to conventional fractionation;

- Hypofractionation can reduce early urinary complication due to lower BED to the urinary system;

- Hypofractionation can provide more convenience for patients;

- Hypofractionation can reduce the demand for treatment resources;

- Individually determined $\alpha / \beta$ will be one of the indicators for potential biologically-based treatment plan optimizations.

In the work presented here, we have adapted the radiobiological parameter values from Wang ${ }^{[155]}$ based on the following considerations. Although clinical evidences indicate that the $\alpha / \beta$ is low, the number of tumour cells assumed by Brenner is very small $(\sim 100$ cells)and is not appropriate for the voxel based TCP calculation. On the other hand, the 
value of $8.4 \mathrm{~Gy}$ for the $\alpha / \beta$ ratio from Nahum conflicts with existing clinical data. Both were not adopted in our simulations.

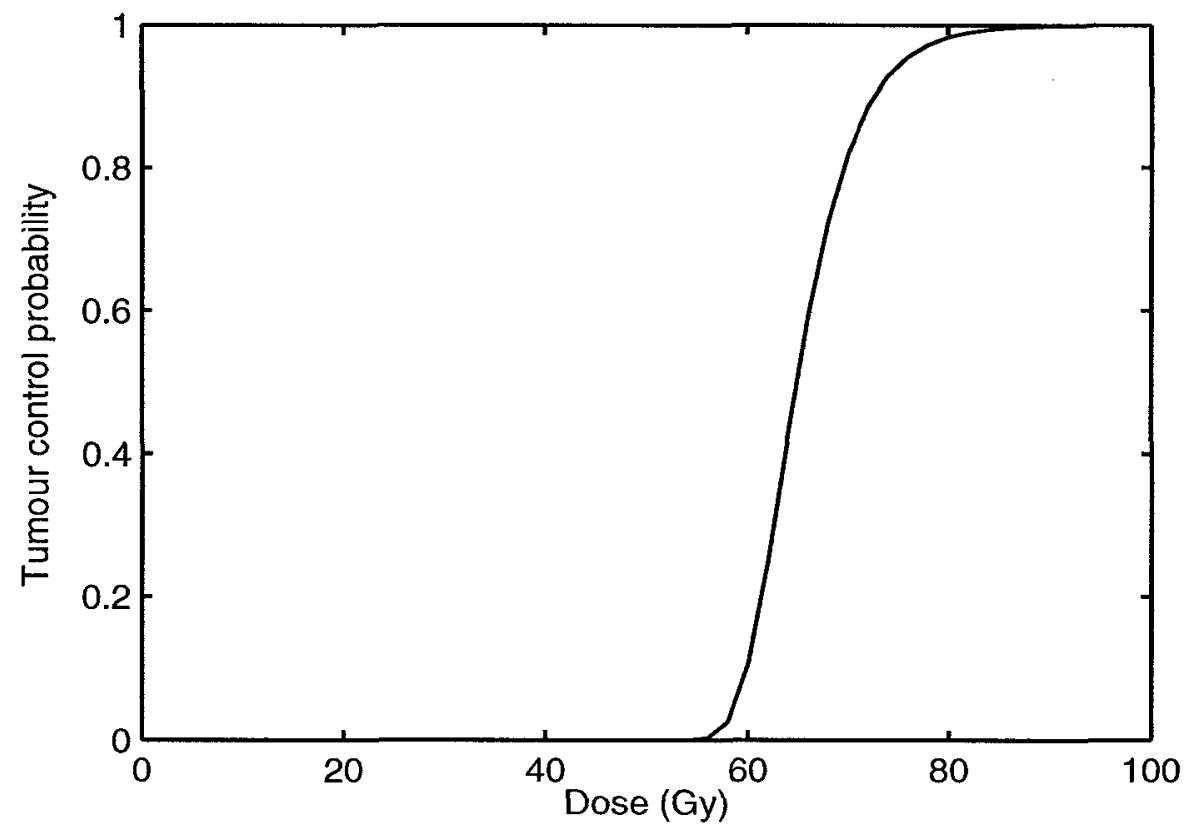

Figure 6.3 TCP as a function of dose

The TCP as a function of total radiation dose using Wang's estimate is illustrated in Figure 6.3 for a fractionated scheme of 2 Gy-per-fraction. Note the number of initial tumour cells is assumed to be $5 \times 10^{6}$. According to the equation 7-4 and 7-11, a value of 0.90 for TCP can be obtained if a radiation dose of $76 \mathrm{~Gy}$ is delivered to the prostate CTV.

\subsubsection{The Dosimetric Consequences of Geometric Error}

The presence of geometric errors in the treatment process not only increases dosimetric uncertainties in the tumour, but also increases the radiation exposure to normal tissues. Methods for dose calculation incorporating geometric uncertainties include convolution and Monte Carlo techniques as discussed below. 


\subsubsection{The Convolution Method}

The convolution method has been used to predict mean dose distribution assuming random uncertainties such as day-to-day patient positioning error and inter-fraction prostate motion ${ }^{[150,157,158]}$. The estimation of mean dose can be accomplished by convolving a static planning dose distribution with a probability distribution function of the geometric errors in three-dimensional space. With the convolution approach and assuming that geometric error has a Guassian distribution, the mean dose distribution D to the tumour target is given by:

$$
D(x, y, z)=\iiint D_{p}\left(x^{\prime}, y^{\prime}, z^{\prime}\right) g_{m}\left(x-x^{\prime}, y-y^{\prime}, z-z^{\prime}\right) d x^{\prime} d y^{\prime} d z^{\prime}
$$

Further, assuming that no correlation exists among the three orthogonal directions, the probability density distribution of errors, can be described as

$$
g_{m}\left(x-x^{\prime}, y-y^{\prime}, z-z^{\prime}\right)=\frac{1}{\sqrt[3]{2 \pi} \sigma_{x} \sigma_{y} \sigma_{z}} \exp \left[-\frac{\left(x-x^{\prime}\right)^{2}}{2 \sigma_{x}^{2}}-\frac{\left(y-y^{\prime}\right)^{2}}{2 \sigma_{y}^{2}}-\frac{\left(z-z^{\prime}\right)^{2}}{2 \sigma_{z}^{2}}\right]
$$

where $\sigma_{x}, \sigma_{y}$ and $\sigma_{z}$ are the standard deviations of the global geometric errors in rightto-left, inferior-to-superior and posterior-to-anterior directions, respectively. The standard deviation of the mean dosimetric error in each dose location is given by:

$$
\sigma_{D}(x, y, z)=\left[\iiint\left[D(x, y, z)-D_{P}\left(x^{\prime}, y^{\prime}, z^{\prime}\right)\right]^{2} g_{m}\left(x-x^{\prime}, y-y^{\prime}, z-z^{\prime}\right)\right]^{1 / 2}
$$

Note that an infinite fraction number is assumed in the convolution technique. However, a realistic fraction number $n_{f}$ usually ranges from 30 to 40 for prostate cancer. The limits of the convolution based dose calculation have been described by Craig. ${ }^{[159]}$ 


\subsubsection{Monte Carlo Technique}

The geometric uncertainty arising in the treatment preparation stage $\left(\boldsymbol{\Sigma}_{T}\right)$ is due to an inaccurate knowledge of the mean tumour position during delivery while the geometric uncertainty in the treatment delivery stage $\left(\sigma_{T}\right)$ is due to the statistical nature of the dayto-day patient positioning error and inter-fraction organ motion. By assuming a global mean position ( 0 for instance) for prostate in the treatment process (including preparation and delivery stages), the prostate position $r_{p}$ with respect to global mean position in planning stage for a patient can be sampled from the distribution defined by the $\Sigma_{T}$ using Monte Carlo technique. Similarly, a daily treatment deviation from the global mean position $\zeta_{i}^{t}$ in delivery stage can also be sampled from the distribution defined by $\sigma_{T}$. Let $N_{f}$ be the number of fractions in a treatment course, the global prostate deviation $\Delta r_{i}$ in fraction $i$ from planned position $r_{p}$ can then be described as:

$$
\Delta r_{i}=\zeta_{i}^{t}-r_{p}
$$

Note that only the position deviation due to a combination of positioning error, intrafraction and inter-fraction prostate motion were simulated in this study. The effects of rotation, target deformation and target delineation were not considered.

For a simulated fraction in a treatment course, the actual dose distribution to the CTV in the presence of geometric error can be calculated with the treatment planning computer (e.g. Theraplan 3.0). Dose accumulation in each voxel of the CTV over all fractions from a treatment course represent the actual dose distribution to the CTV. However this approach is time consuming (20 mins per simulated fraction with our planning computer), and is not realistic for a simulation of a large treatment courses. This approach can be eliminated because our clinical measurements indicate that the variation in the dose to the CTV is less than $1 \%$ in $95 \%$ of cases where the treatment displacement is less than $12 \mathrm{~mm}$. This suggests that the actual dose distribution in prostate CTV can be 
estimated by shifting the planned dose distribution in CTV from its planned position to the daily treated position in its original coordinate system.

We assume that the dose distribution to the CTV changes with the daily global geometric error. In order to calculate the dose to the voxels of prostate CTV during the treatment, the dose deposited to the moving voxels is translated to the stationary voxels in the original coordinate system (or CTV in the planned CT coordinate system). Thus the fraction of dose delivered to voxels of the prostate CTV is still in the dose coordinates, $D^{C T V} \in D_{P}$. This shifts the dose from

$$
D\left(r_{p}\right) \Leftarrow D_{P}\left(r_{p}-\Delta r_{i}\right)
$$

where $\Delta r_{i}$ is the global geometric error of prostate CTV and $r_{p}$ is the CTV position in its original coordinate system. Dose accumulated to the voxels of prostate CTV over 38 fractions represents the actual dose distribution in the CTV for a simulated treatment.

\subsubsection{The Biological Effect}

The effect of geometric error on the local control probability was quantified with TCP after dose reconstruction in the CTV. Assuming that cell killing amongst tumour voxels is uncorrelated and only depends on the actual dose to a particular voxel, the TCP for the entire tumour is given by

$$
T C P=\prod_{i=1}^{N_{f}} \exp \left(-\iiint_{C T V} \rho_{c} C T V(x, y, z) e^{-\alpha d_{d e l}(x, y, z)-\beta d_{d e l}^{2}(x, y, z)} d x d y d z\right) .
$$

where

$$
C T V(x, y, z)=\left\{\begin{array}{ccc}
1 & \text { inside } & C T V \\
0 & \text { outside } & C T V
\end{array}\right.
$$

where $\rho_{c}$ is the homogenous tumour cell density. 
The EUD was determined for the CTV using the equations from 6.6 to 6.12 while the NTCP for the rectal wall was calculated using eq. 6-15 thru 6-18. The flowchart of the simulation is illustrated in Figure 6.4.

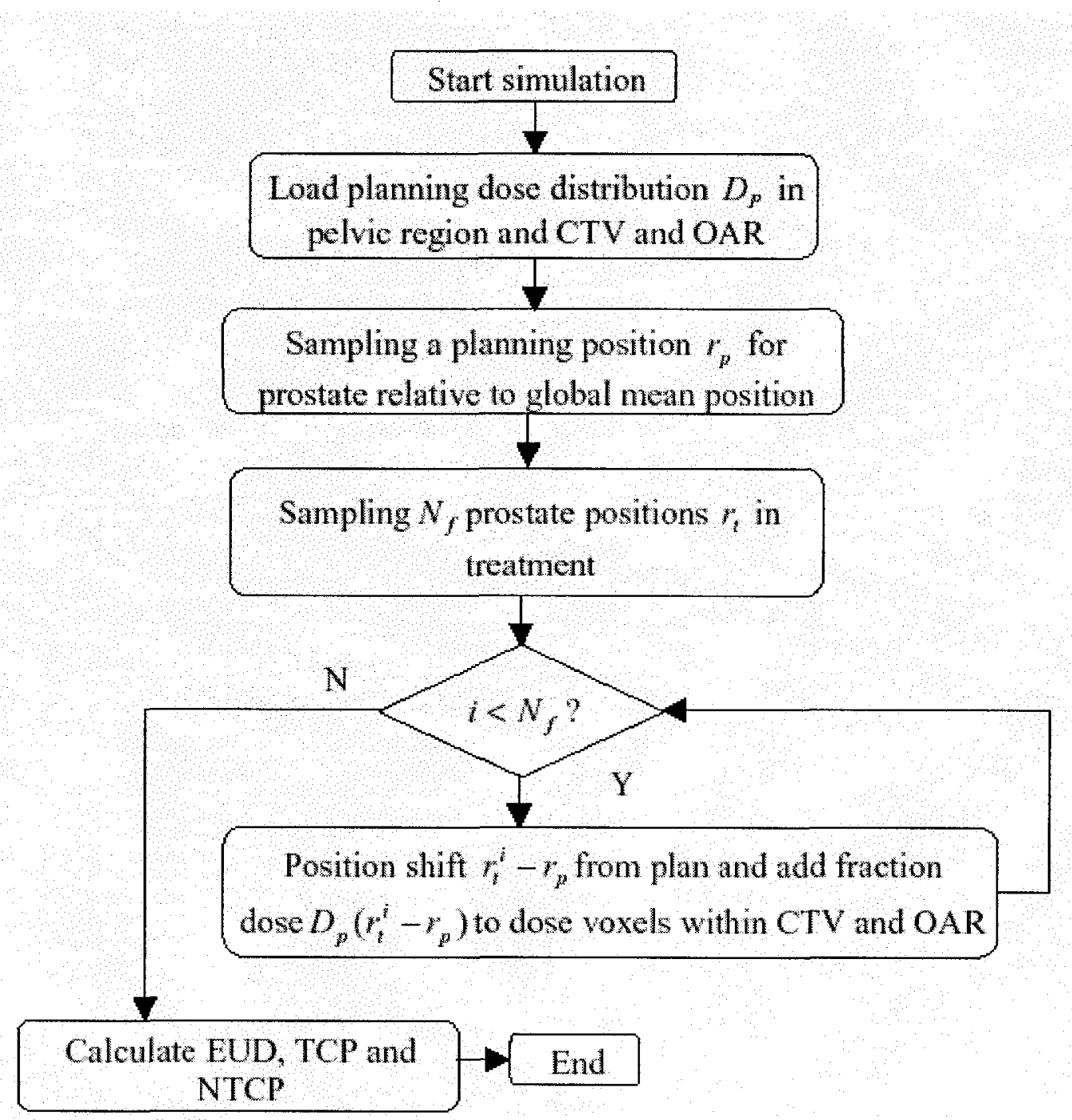

Figure 6.4 Flowchart of the treatment simulation in the presence of geometric errors

\subsection{Results}

\subsubsection{Patient and Treatment Planning}


Clinical data for a six-field XRT prostate cancer patient at TOHRCC were extracted and utilized in this simulation.. Our treatment protocol for six-field XRT technique for intermediate risk patients consists of a total of 76 Gy delivered over 38 daily fractions. The initial component is $56 \mathrm{~Gy}$ with a boost of $20 \mathrm{~Gy}$. The error data comes from our study of patients treated either in a prone position with a Hip-Fix immobilization or in a supine position without fixation. The treatments were delivered with18 MV photons from a Siemens Digital Mevatron linear accelerator. The planning target volume was formed by adding a uniform margin of $10 \mathrm{~mm}$ to the $95 \%$ isodose line of the CTV in the transverse plane for the initial and boost volumes (except a margin of $5 \mathrm{~mm}$ was used for the posterior part of the CTV). This is the presently accepted TOHRCC method to mitigate against the effect of geometric uncertainty, In addition, for the initial volume, 15 and $20 \mathrm{~mm}$ margins are added to the superior and inferior direction respectively. These margins are reduced to 10 and $15 \mathrm{~mm}$ for the boost volume. Under the beam arrangements outlined above, planning dose distributions within the pelvis for two different margins were calculated with a $2 \times 2 \times 2 \mathrm{~mm}$ voxel size using our planning software (Theraplan Plus version 3.0, Nucletron, Netherlands). Both the CTV and OAR volumes were extracted from the planning CTs and exported as Microsoft Excel files for radiobiological modeling. Additionally, planned dose distributions were exported to

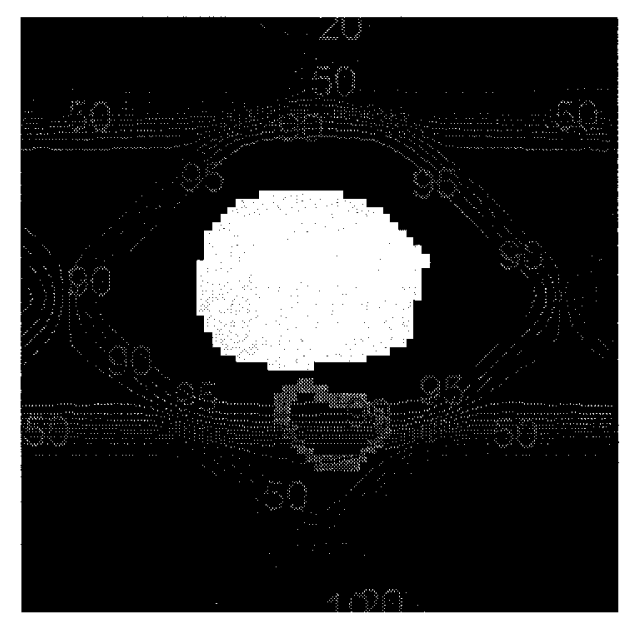

Figure 6.5 Planned dose distribution in the pelvic region. The white represents the prostate CTV while the gray represents the rectal wall. 
Matlab 6.5 for dosimetric analysis. The rectal wall was the only OAR defined for normal tissue toxicity analysis. As an example, Figure 6.5 illustrates the planned isotropic dose distribution in the pelvic region. According to the radiobiological parameters suggested by Wang, a TCP of 0.90 can be achieved if the dose is delivered to the CTV as planned. The NTCP for the rectal wall was calculated to be 0.02 under the prescribed dose.

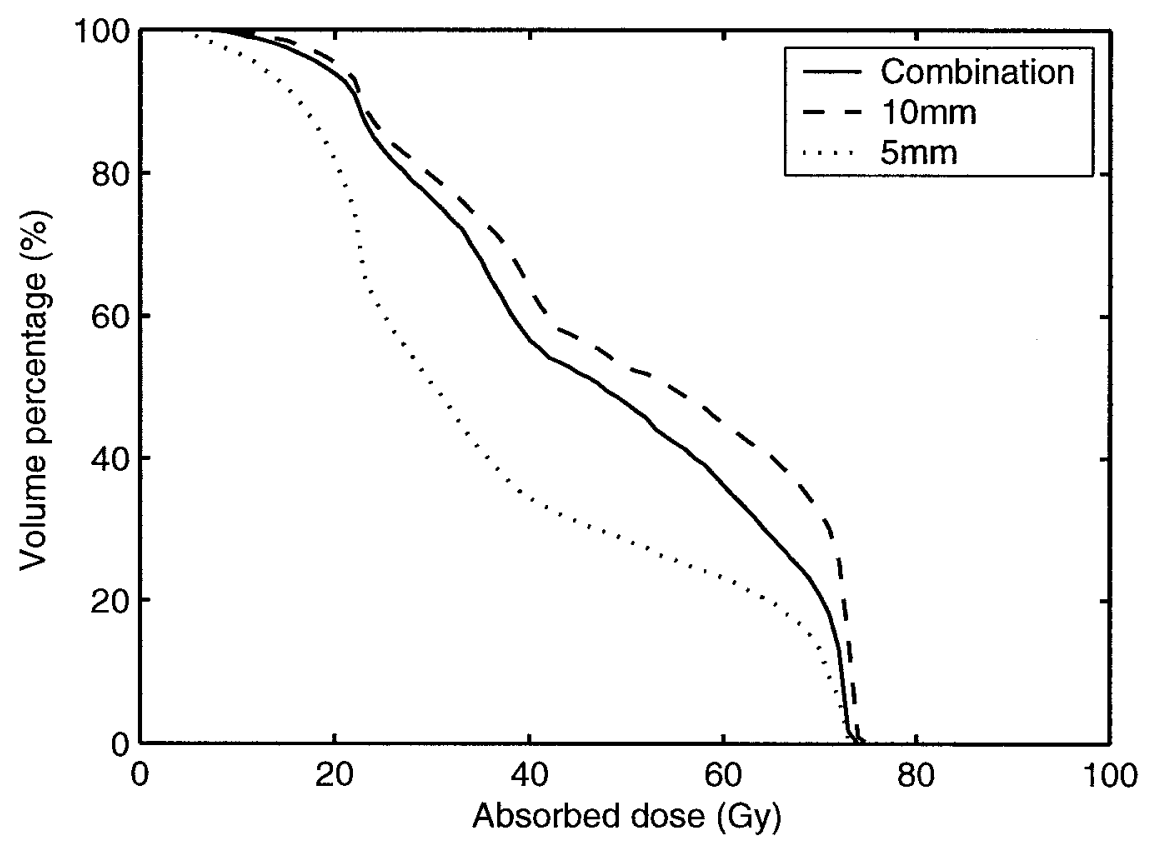

Figure 6.6 Dose-volume histograms for rectal wall

Figure 6.6 and 6.7 are dose-volume histograms for the rectal wall and the prostate CTV for a treatment with a margin of $10 \mathrm{~mm}$, anisotropic $5 \mathrm{~mm}$ and a combination of both ("Combination"). Figure 6.7 shows nearly identical DVH curves for prostate CTV for the three treatment scenarios. But as shown in Figure 6.6, the DVH for the rectal wall differs significantly. 


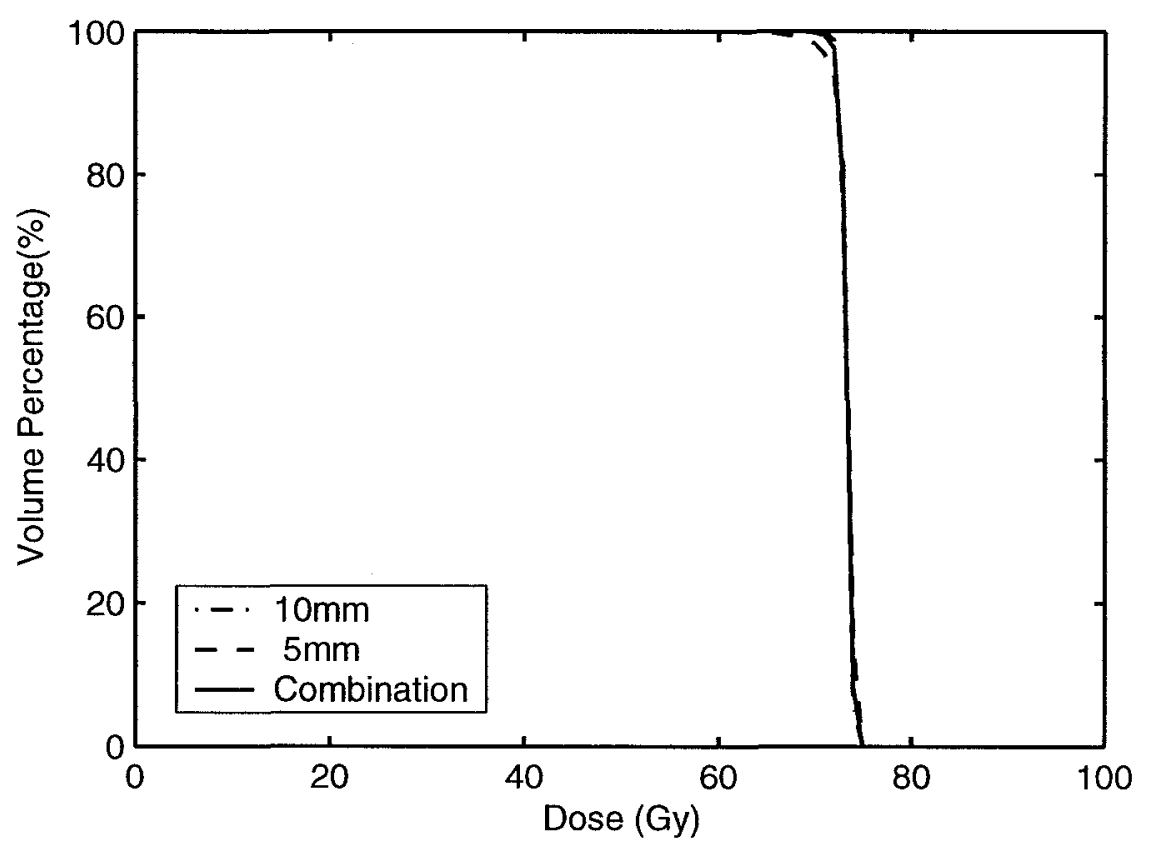

Figure 6.7 Dose-volume histograms for the prostate CTV.

TCP is predicted in the presence of geometric errors for the three different 3DCRT techniques. First it was evaluated for the treatment without clinical intervention, second with positioning error correction, and third with full geometric error correction. The prescribed dose was varied from 50 to $100 \mathrm{~Gy}$ in $2 \mathrm{~Gy}$-per-fraction increments. For each prescribed dose, 200 treatment courses were simulated for both $10 \mathrm{~mm}$ and $5 \mathrm{~mm}$ anisotropic margins. The TCP, NTCP and EUD values were subsequently calculated. For each simulated course, the overall systematic error, positioning error and inter-fraction target motion were independently sampled (see Table 6.1). The actual dose distribution in the prostate CTV and the rectal wall were accumulated over each fraction in a simulated treatment course. NTCP was calculated using the Lyman model, where the effective volume was calculated using Eq. 6.16 based on the actual acquired dose-volume histogram from each simulated course. 


\subsubsection{The Effect of Geometric Errors}

For treatment without clinical intervention to correct for the geometric errors, Figure 6.8 (a) and (b) plot the TCP and the NTCP as a function of prescription dose, respectively. Figure 6.8 (a) demonstrates that the TCP increases with the radiation dose for 10 and $5 \mathrm{~mm}$ anisotropic margins. There are considerable TCP differences between treatments with anisotropic $5 \mathrm{~mm}$ and $10 \mathrm{~mm}$ margins. As an example, TCP decreases from 0.82 to 0.70 when the margin shrinks from $10 \mathrm{~mm}$ to $5 \mathrm{~mm}$ if a prescription dose of 76 Gy is applied. Correspondingly, as shown in Figure 6.8(b), the rectal toxicity is reduced from 0.06 to 0.01 .

With the second technique, the day-to-day positioning error was corrected. Figure 6.9(a) and (b) are plots of T.CP and NTCP, respectively for treatment with two designated margins and as well as for a combined treatment (25 fractions were treated with $10 \mathrm{~mm}$ margin, else with $5 \mathrm{~mm}$ margin). The TCP decreases 0.12 when the margin is reduced from $10 \mathrm{~mm}$ to $5 \mathrm{~mm}$ for a total dose of $76 \mathrm{~Gy}$.

For the third technique, it was assumed that the geometric error had been fully corrected before each fraction. Figure 6.10 illustrates both TCP and NTCP for three margins. The figure demonstrates there is little difference in the TCP curves between the two margins. However, NTCP for $5 \mathrm{~mm}(0.01)$ margin is superior to the $10 \mathrm{~mm}(0.06)$ margin.

There is little NTCP difference among the three treatment techniques if the same margin is applied. This implies that the rectal toxicity is more dependent on treatment margin than on treatment technique. However, the TCP depends both on treatment technique and margin. 


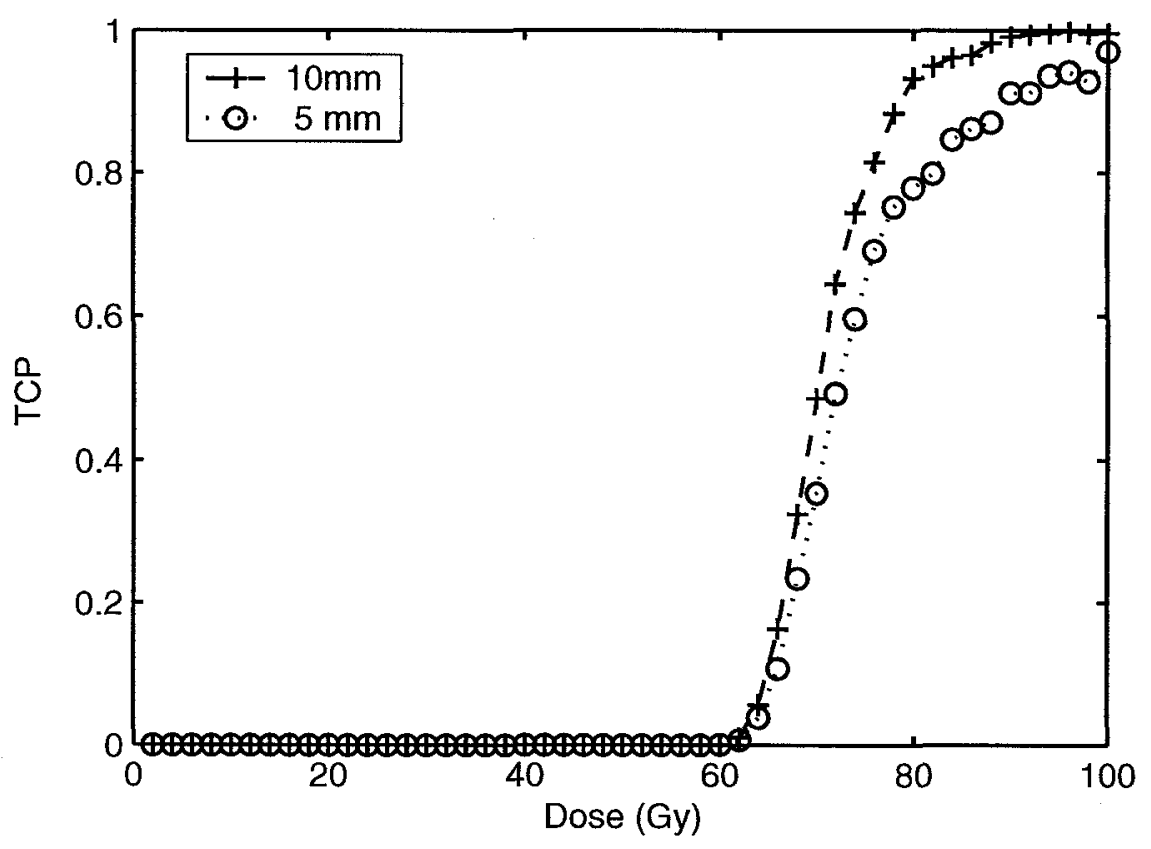

Figure 6.8(a) TCP as a function of prescription dose for two typical margins (No clinical intervention applied)

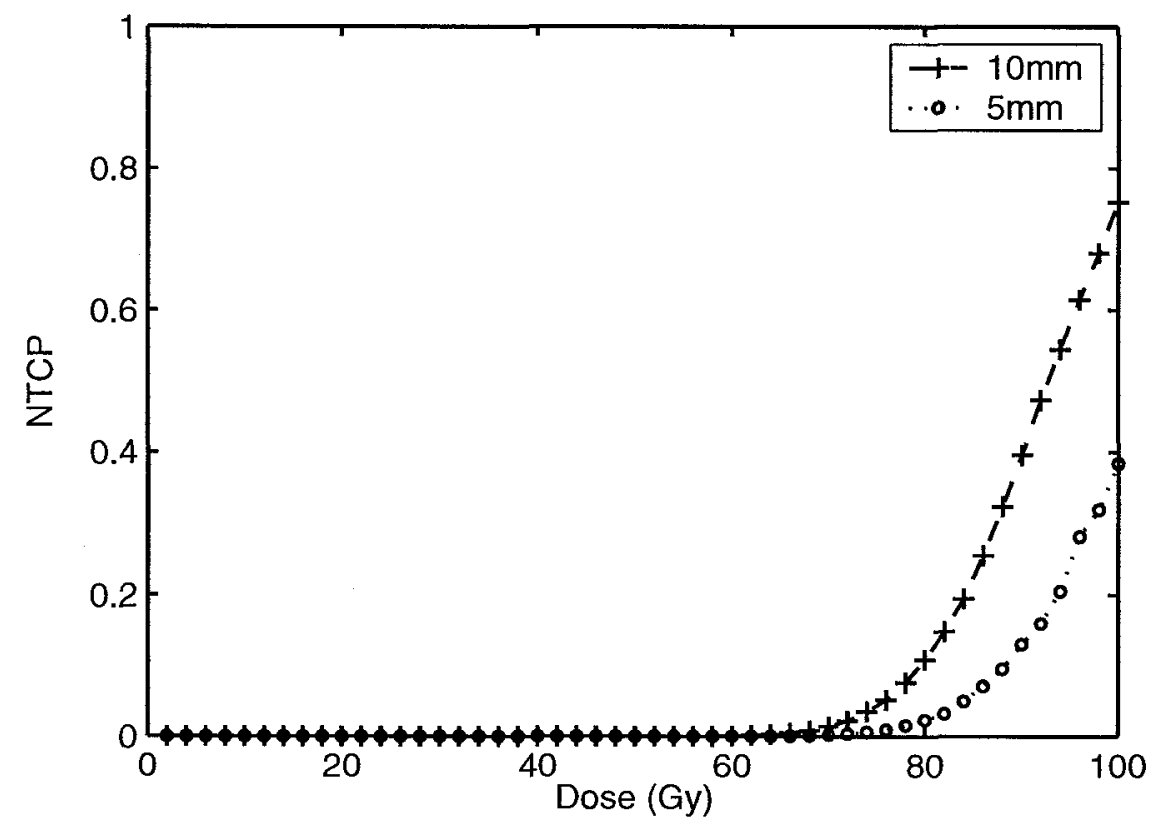

Figure 6.8(b) NTCP as a function of prescription dose for two typical margins (No clinical intervention applied) 


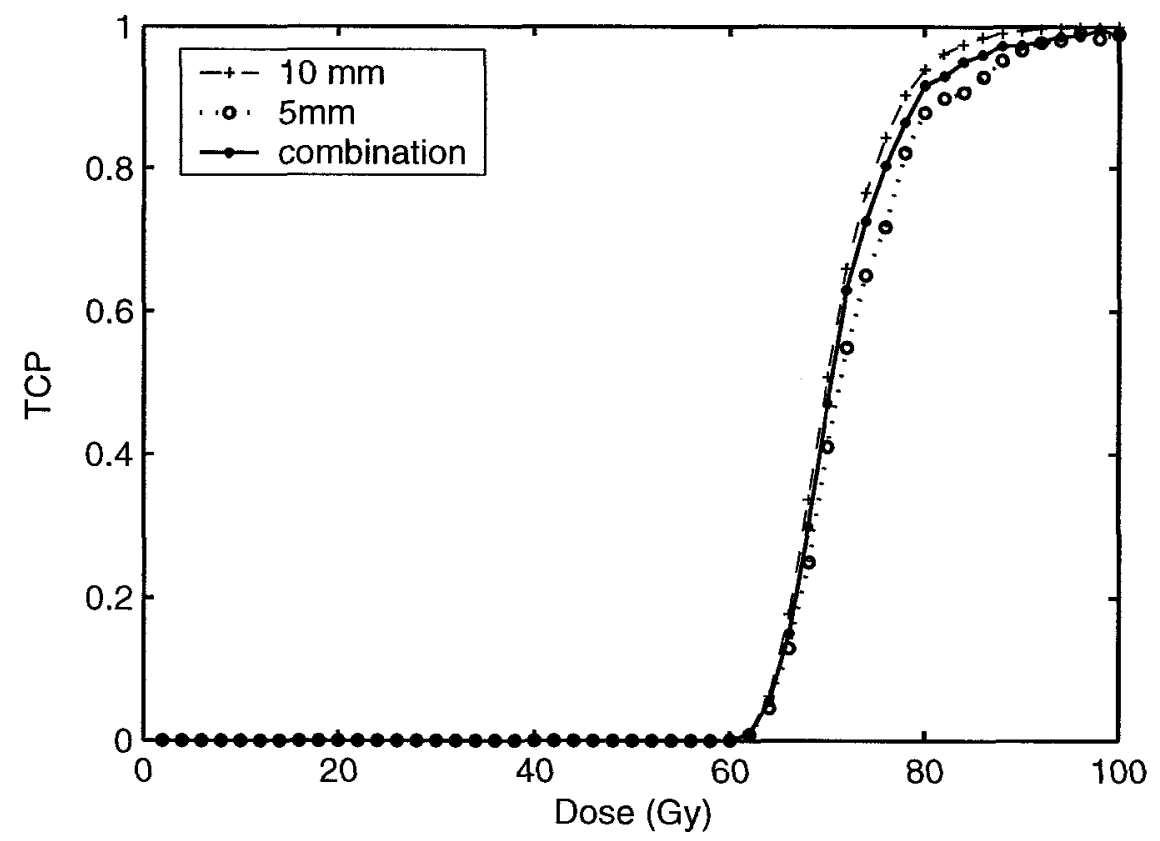

Figure 6.9(a) TCP as a function of radiation dose for three scenarios (Positioning error corrected)

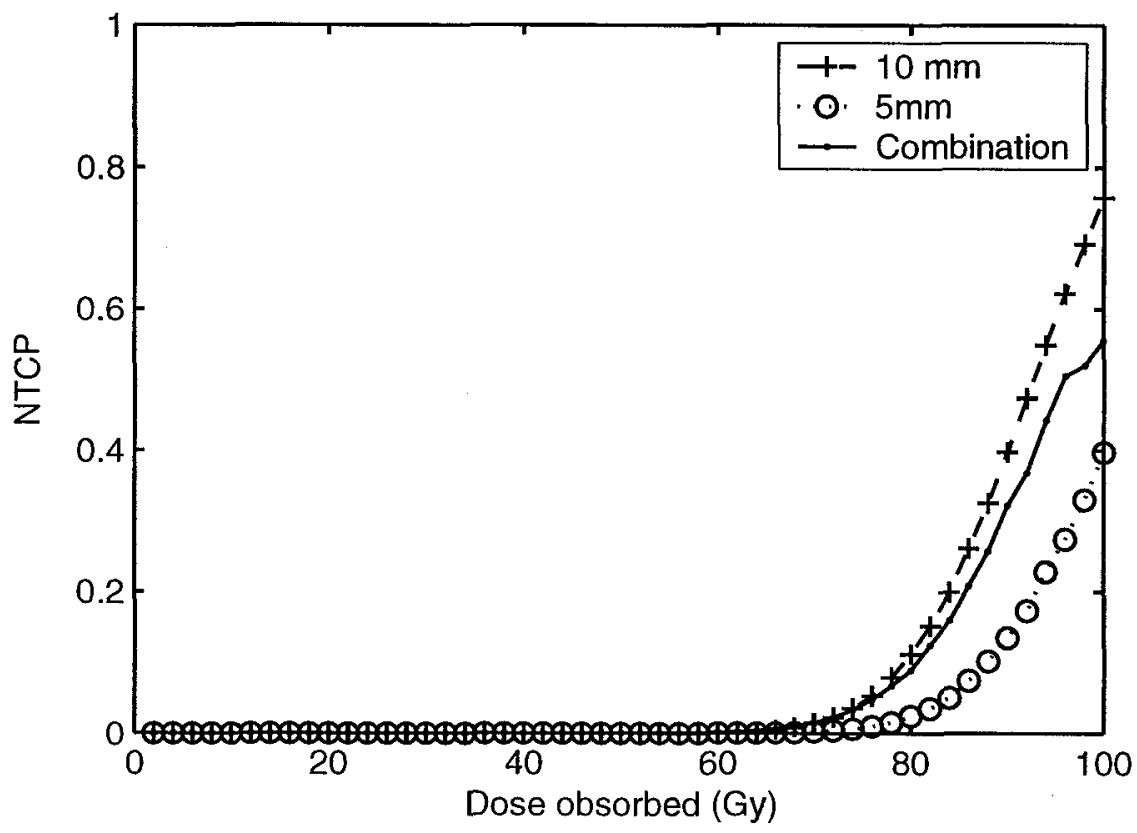

Figure 6.9(b) NTCP as a function of radiation dose for two typical margins (Positioning error corrected) 


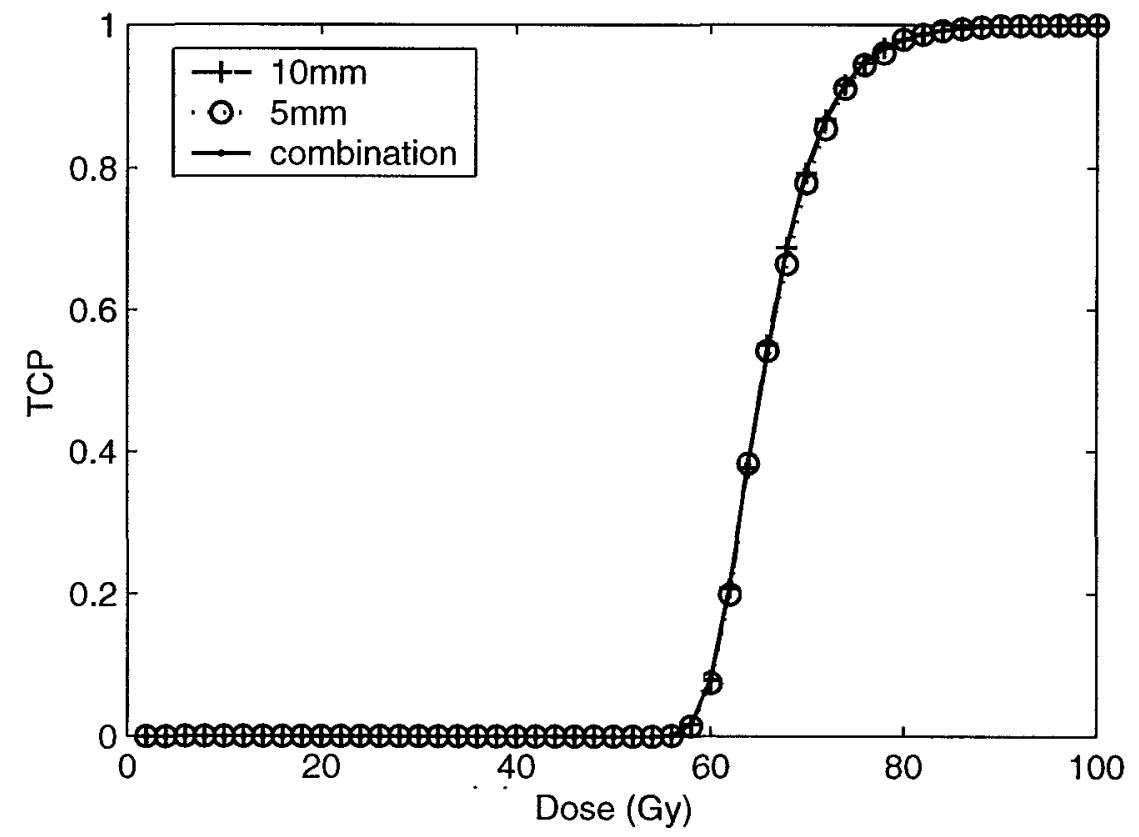

Figure 6.10(a) TCP as a function of radiation dose for two typical margins (full geometric error corrected)

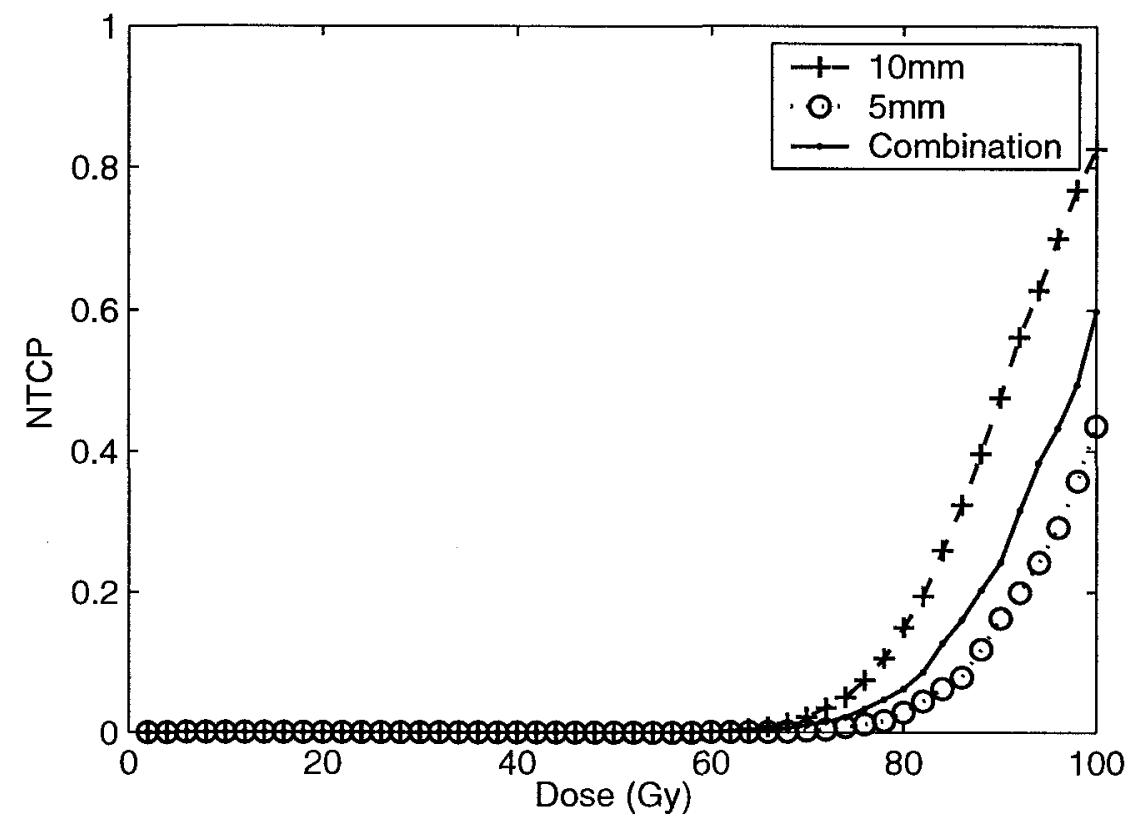

Figure 6.10(b) NTCP as a function of radiation dose for two typical margins (Full geometric error corrected) 


\subsubsection{The Effect of Systematic Error}

The effect of the individual systematic error on the local tumour control was also investigated. In this simulation, it was assumed that $0,20,40,60,80$ and $100 \%$ of the uncertainty arising in the preparation stage, or standard deviation of individual systematic error (see table 6.2) can be corrected. TCP as a function of systematic error was calculated for our 3DCRT technique and the results are shown in Figure 6.11. Note, 2000 treatment courses were simulated for each systematic error. Figure 6.11 demonstrates that the TCP improves as more of the systematic error is removed. Additionally, the uncertainty in TCP decreases with the reduction in the individual systematic error. For instance, the TCP would improve from 0.82 to 0.85 if $40 \%$ of the individual systematic error was removed. Similarly, the effect of random uncertainty was simulated. We found that random uncertainties alone had little impact on. the TCP for the initial treatment with $10 \mathrm{~mm}$ margin.

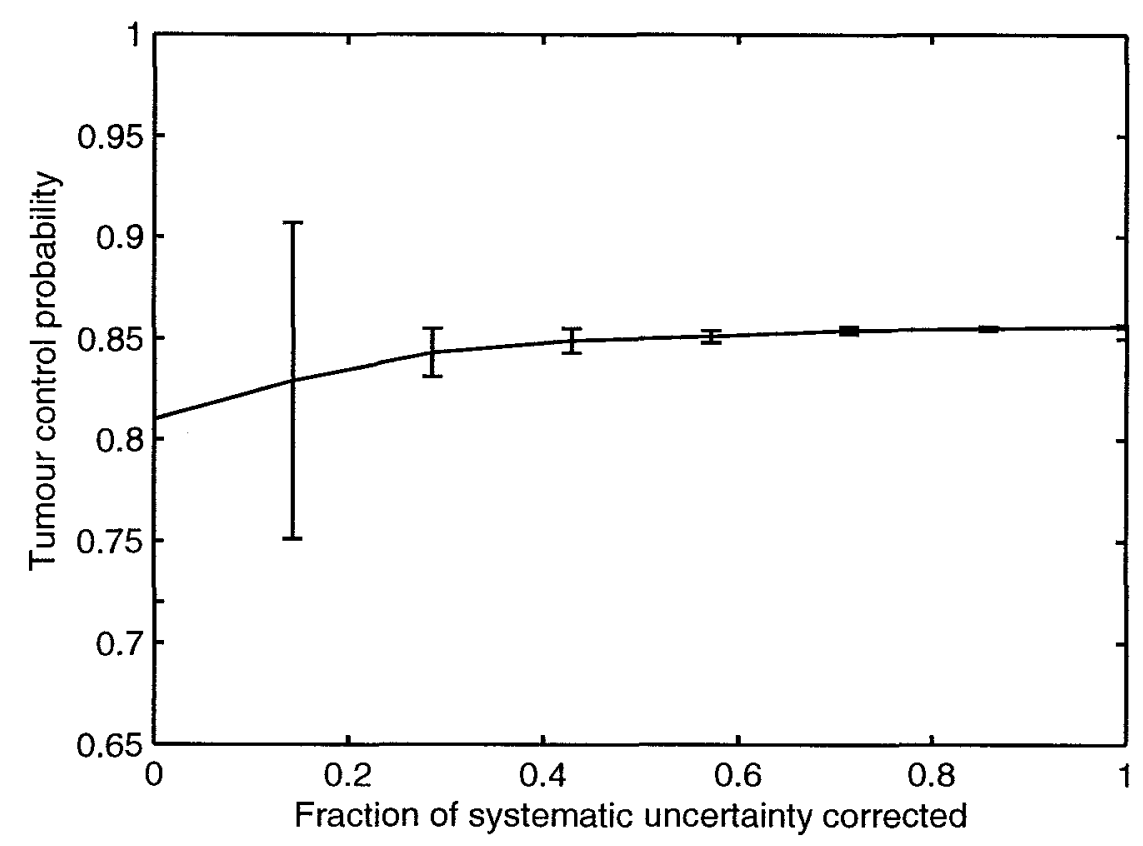

Figure 6.11 TCP as a function of systematic uncertainty 


\subsection{Discussion}

\subsubsection{General Discussion}

For a sophisticated treatment process such as prostate cancer radiotherapy, patient outcome is generally affected by the delivery equipment related uncertainty (e.g. CT and linac), patient related uncertainty (e.g. target definitions, positioning error, tumour motion, etc), and tumour response to the radiation and delivery patterns. Each has been intensively investigated and reported in the literature (see Chapter 2 for detail). The smallest of the geometric uncertainties $(\sim 1 \mathrm{~mm})$ is contributed by the simulation and delivery equipment. Human related uncertainty, on the other hand, has been measured and well quantified with reasonable accuracy. We have demonstrated that the magnitude of the patient related uncertainty $(5 \sim 6 \mathrm{~mm})$ is significantly larger than the machine related uncertainty. It is also commonly agreed that the uncertainty in radiobiological parameters is a signficant source of uncertainty contributing to the treatment quality, but has not been sufficiently emphasized in previous studies. The challenge faced in this study is the lack of knowledge of tumour and normal tissue responses to radiation and its delivery patterns. In the radiotherapy community, we are more interested in the effect of treatment related uncertainty for prostate cancer, in particular machine and patient-related uncertainties. The machine related uncertainty is ignored in this study because of it has been shown here to be significantly smaller than human related uncertainties. in. Tumour heterogeneity and inter-patient radiosensitivity were not considered.

\subsection{2 $\alpha / \beta$ ratio and Hypofractionation}

In the radiobiological modeling of prostate cancer, the choice of $\alpha / \beta$ ratio is always a question that must be addressed because it can significantly affect the conclusions.

If it is true that the $\alpha / \beta$ ratio for prostate cancer is significantly lower than that for typical tumours (8 10 Gy) or late-responding normal tissues (e.g. rectum) (3 4 Gy), prostate cancer would then be very sensitive to changes in the fraction size. In hypofractionation, a large fraction size ( $>2$ Gy per fraction) is delivered daily and the 
total number of fractions is reduced. The use of hypofractionated regimens could be beneficial to prostate patients in terms of convenience and could also be useful to the cancer programs in terms of resource utilization. However, the implementation of hypofractionation for prostate cancer introduces several difficulties. First, early normal responding tissues must be taken into account when a hypofractionation strategy is developed ${ }^{[148]}$. Secondly, the geometric uncertainties and corresponding treatment margins can also affect outcome as has been shown by $\mathrm{Craig}^{[99]}$ and other investigators. $^{[142]}$ The biological effect of underdosage is more dramatic in hypofractionation schemes than in conventional techniques. Several groups have already used various hypofractionated methods to treat prostate cancer patients. This includes a wide range of dose-per-fraction $(2.5-6 \mathrm{~Gy})^{[160-162]}$, treatment times (up to 8 weeks) and boost techniques.

Instead of using an extremely low $\alpha / \beta$ value (1.5) for prostate cancer ${ }^{[99,142]}$, we assumed a moderate value of 3.1 for both rectal wall and prostate CTV. The effect of the geometric uncertainty in combination with two margins was compared for treatments with three fractionation schemes $38 \times 2.0 \mathrm{~Gy}, 21 \times 3.0 \mathrm{~Gy}$ and $7 \times 6.1 \mathrm{~Gy}$. Note all three schemes are approximately equivalent to a dose of 76 Gy delivered with 2 Gy perfraction, or a EUD dose of $123 \mathrm{~Gy}$. Each scheme was simulated 2000 times for two different margins as previously described. The NTCP for the rectal wall and the TCP for the prostate CTV are summarized in table 6.4 and 6.5 , respectively.

The simulation for different schemes suggests that TCP is affected by both margin and treatment technique for all three fractionation schemes. Our simulation shows that the TCP for the hypofractionation scheme $(7 \times 6.1 \mathrm{~Gy})$ is more sensitive to margins than the conventional schemes. For the hypofractionated treatment (6.1 Gy per fraction) without correction for geometric error, the TCP decreases by 0.21 when the margin is reduced. However TCP decreases by only 0.12 if a conventional scheme is utilized. This implies that the treatment margin for hypofractionated schemes must be carefully considered before it is implemented. Rectal toxicity does not change significantly for the various fractionation schemes. This implies that there is no benefit in terms of rectal toxicity 
when a hypofractionation scheme is utilized. This conclusion differs from what has been reported by Craig. In his study, NTCP for rectum could be significantly reduced if the conventional scheme is replaced with the hypofractionation scheme. This difference between Craig's conclusions and ours arises from the difference in fundamental assumption of $\alpha / \beta$ value. Craig assumed an extremely low $\alpha / \beta$ value of 1.5 for prostate and a moderate value of 3 for rectal wall. His simulation goal was to investigate the therapeutic benefit of prostate treatment with hypofractionation. For such a low value for $\alpha / \beta$, the prostate was more sensitive to a change in the number of fractions than the rectum. However, we applied a comparable $\alpha / \beta$ value for both prostate and rectal wall to investigate the effect of geometric uncertainty. As expected we found that no therapeutic benefit can be obtained by changing the fraction size.

As simulated by Craig, a low $\alpha / \beta$ value would be attractive for the treatment of prostate cancer with a hypofractionation scheme because tumour control can be significantly improved while normal tissue complications are relatively unchanged. As an example, a hyperfractionated scheme of $3 \times 6.0$ Gy can theoretically achieve a TCP that is equivalent to a conventional scheme of $38 \times 2 \mathrm{~Gy}$. However, the mean BED dose to rectum is significantly reduced to $17 \mathrm{~Gy}$ from $63 \mathrm{~Gy}$ when a conventional scheme is replaced by the hypofractionated scheme. Hypofractionated treatment schemes have a better therapeutic ratio than conventional schemes in terms of sparing normal tissue complications.

The hypofractionated scheme is superior to a conventional scheme only if the $\alpha / \beta$ value for prostate cancer is lower than that for normal tissues. Although there is evidence that the $\alpha / \beta$ ratio for prostate cancer is lower than for most tumours ( $>8 \mathrm{~Gy}$ ), the lower limit is still heatedly debated in the radiation biology community. Most arguments regarding the $\alpha / \beta$ value for prostate cancer arise as follows. First, the validity of the relationship between TCP and the biological endpoints needs to be verified. For instance, a biological marker such as freedom from biochemical failure at 3 years was used as define successful tumour control (TCP) in several publications. The resulting low $\alpha / \beta$ value becomes 
questionable if it appears a different endpoint (e.g. freedom from biochemical failure at 5 or 10 years) would be more indicative of tumour control. Second, a large uncertainty exists in the clinical data among different population groups, institutions, protocols and treatment methods. These uncertainties are critical to the determination of the $\alpha / \beta$ value. Third, most methods for determining the $\alpha / \beta$ value also involve an estimation of the number of initial cells $N_{0}$ and $\alpha$, which also may bring uncertainty.

Table 6.4 Summary of NTCP for different fractionated schemes

\begin{tabular}{||c|c|c|c|c|c|c|}
\hline \multirow{2}{*}{ Schemes } & \multicolumn{2}{|c|}{ No error corrected } & \multicolumn{2}{c|}{ Setup error corrected } & \multicolumn{2}{c|}{ Full error corrected } \\
\cline { 2 - 7 } & $10 \mathrm{~mm}$. & $5 \mathrm{~mm}$ & $10 \mathrm{~mm}$ & $5 \mathrm{~mm}$ & $10 \mathrm{~mm}$ & $5 \mathrm{~mm}$ \\
\hline $38 \times 2.0 \mathrm{~Gy}$ & $0.05 \pm 0.01$ & $0.010 \pm 0.005$ & $0.055 \pm 0.009$ & $0.010 \pm 0.004$ & $0.055 \pm 0.004$ & $0.011 \pm 0.002$ \\
\hline $21 \times 3.0 \mathrm{~Gy}$ & $0.05 \pm 0.01$ & $0.008 \pm 0.004$ & $0.051 \pm 0.008$ & $0.009 \pm 0.004$ & $0.049 \pm 0.003$ & $0.010 \pm 0.002$ \\
\hline $7 \times 6.1 \mathrm{~Gy}$ & $0.07 \pm 0.01$ & $0.013 \pm 0.006$ & $0.066 \pm 0.011$ & $0.013 \pm 0.005$ & $0.068 \pm 0.005$ & $0.014 \pm 0.002$ \\
\hline
\end{tabular}

Table 6.5 Summary of TCP for different fractionated schemes

\begin{tabular}{||c|c|c|c|c|c|c||}
\hline \multirow{2}{*}{ Schemes } & \multicolumn{2}{|c|}{ No error corrected } & Setup error corrected & \multicolumn{2}{c||}{ Full error corrected } \\
\cline { 2 - 7 } & $10 \mathrm{~mm}$ & $5 \mathrm{~mm}$ & $10 \mathrm{~mm}$ & $5 \mathrm{~mm}$ & $10 \mathrm{~mm}$ & $5 \mathrm{~mm}$ \\
\hline $38 \times 2.0 \mathrm{~Gy}$ & $0.82 \pm 0.10$ & $0.70 \pm 0.28$ & $0.85 \pm 0.05$ & $0.72 \pm 0.21$ & $0.860 \pm 0.004$ & $0.840 \pm 0.004$ \\
\hline $21 \times 3.0 \mathrm{~Gy}$ & $0.79 \pm 0.13$ & $0.62 \pm 0.29$ & $0.82 \pm 0.04$ & $0.69 \pm 0.23$ & $0.830 \pm 0.003$ & $0.800 \pm 0.003$ \\
\hline $7 \times 6.1 \mathrm{~Gy}$ & $0.84 \pm 0.13$ & $0.63 \pm 0.33$ & $0.86 \pm 0.06$ & $0.71 \pm 0.26$ & $0.880 \pm 0.005$ & $0.830 \pm 0.009$ \\
\hline
\end{tabular}

\subsubsection{Adaptive Radiotherapy}

Dose escalation to the prostate CTV is a challenge in the presence of geometric errors owing to patient positioning variation and inter-fraction prostate motion during the preparation and delivery stages. In principle, dose escalation can be achieved only if the treatment margin is reasonably reduced. However, further margin reduction requires that geometric uncertainty be correspondingly reduced. Several strategies including adaptive radiotherapy techniques have been proposed to correct for the treatment geometric error. 
These techniques include image-guided techniques and online target repositioning techniques and have been shown to reduce a significant proportion of the geometric errors $^{[163]}$. The remaining uncertainty can be addressed with a relatively small margin. However, the clinical use of image-guided techniques for prostate cancer is expensive the cost of an IGRT unit such as Tomotherapy HiArt is 3 4 million dollars, twice the cost of a conventional linac. The maintenance and operation of a Tomotherapy unit requires a large amount of QA workload along with sophisticated calibration procedures. Image Guided treatment delivery takes significantly longer than normal treatment delivery. For example in the experience of the TOHRCC, it takes approximately 12 minutes to setup and delivery a conventional six-beam prostate treatment, but approximately 30 minutes is required to set up, image register and delivery treatment with the Tomotherapy unit. Because the patient throughput of the IGRT is very low, most IGRT devices are only used for research purposes and clinical trials. Commercialization of IGRT technologies requires both efficiency improvements and a reduction in treatment costs.

We have demonstrated that the TCP would be improved if the systematic error could be reduced. We also know that the systematic error is mainly due to an inaccurate knowledge of the mean prostate position prior to radiation delivery. In the adaptive radiation therapy approach, treatment can be delivered using IGRT techniques for the first several fractions. Each of these fractions could then be used to estimate the mean prostate position and apply this mean correction to the reminder of fractions, delivered without image guidance. The adaptive therapy procedure was described in chapter 4 .

The following scenario was simulated. In the first $n_{I G R T}$ fractions, a patient is treated with the image guidance using a $5 \mathrm{~mm}$ margin, which assumes that full geometric error is corrected before radiation delivery. The mean prostate position is then recalculated following $n_{I G R T}$ measurements. The isocentre is then shifted to reflect the updated mean prostate position. The remaining fractions of radiation were subsequently delivered with a conventional technique using a $10 \mathrm{~mm}$ margin. In total, we simulated 2000 instances for each $n_{I G R T}$ (from 1 to 38 ). The mean TCP and NTCP as a function of $n_{I G R T}$ are shown in 
Figures 6.12 and Figure6.13, respectively. For instance, an $N_{I G R T}=0$ means that all treatments are delivered without correction and with a $10 \mathrm{~mm}$ margin. On the other hand, an $N_{\text {IGRT }}=38$ means that all fractions are delivered with IGRT and with $5 \mathrm{~mm}$ margin. For $0<N_{I G R T}<38$, the adaptive strategy discussed above is applied. The data of Figure 6.12 suggest that a treatment is optimized if $7 \sim 10$ fractions of radiation are delivered with image-guidance and the remaining fractions are delivered with conventional techniques, but with the systematic error corrected. The change in TCP as a function of $n_{\text {IGRT }}$ as shown in Figure 6.12 can be explained as follows. TCP is more sensitive to systematic error (as per Van Herk) and hence to the margin than it is to random geometric uncertainty. When a larger portion of the treatments are delivered with IGRT, the estimated mean prostate position gets closer to its actual mean position. Consequently, the reduction in systematic uncertainty leads to TCP increase. As $n_{\text {IGRT }}$ increases, the systematic error approaches zero, which leaves no potential to increase TCP by reducing geometric uncertainty. Meanwhile, the reduced margin tends to decrease TCP.

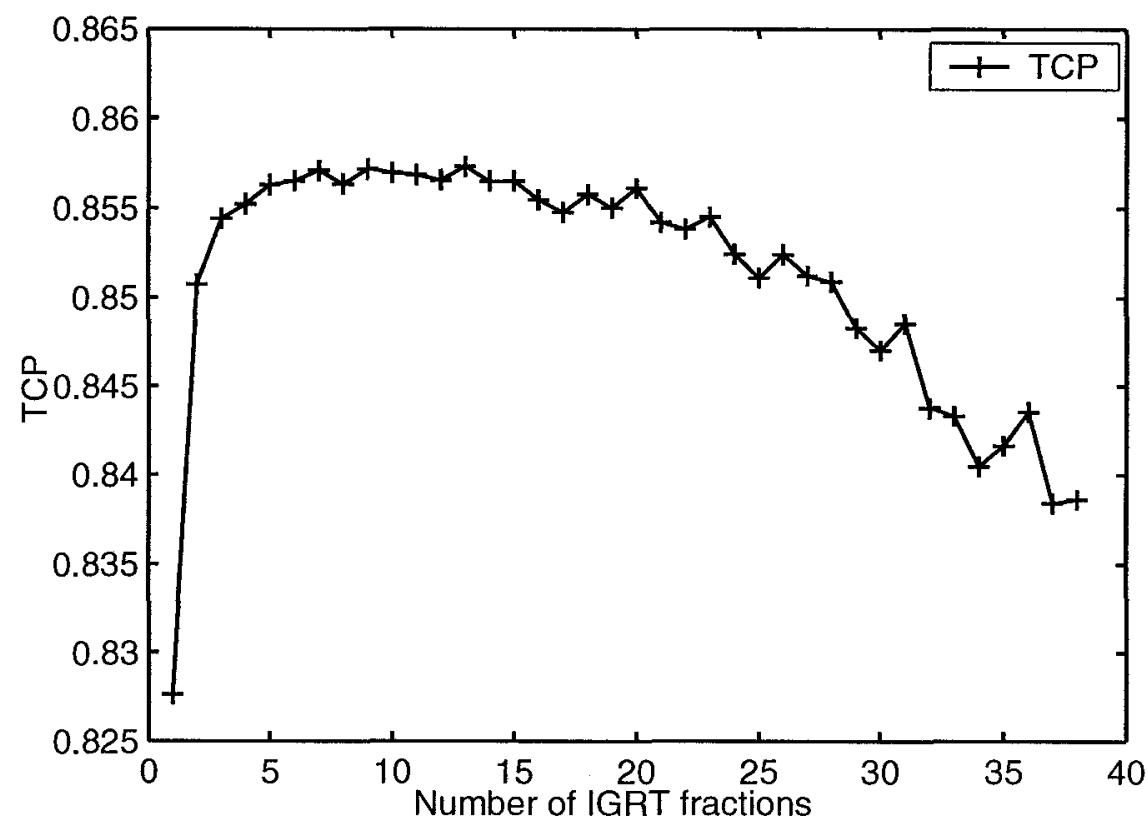

Figure 6.12 TCP as a function of $N_{\text {IGRT }}$ 


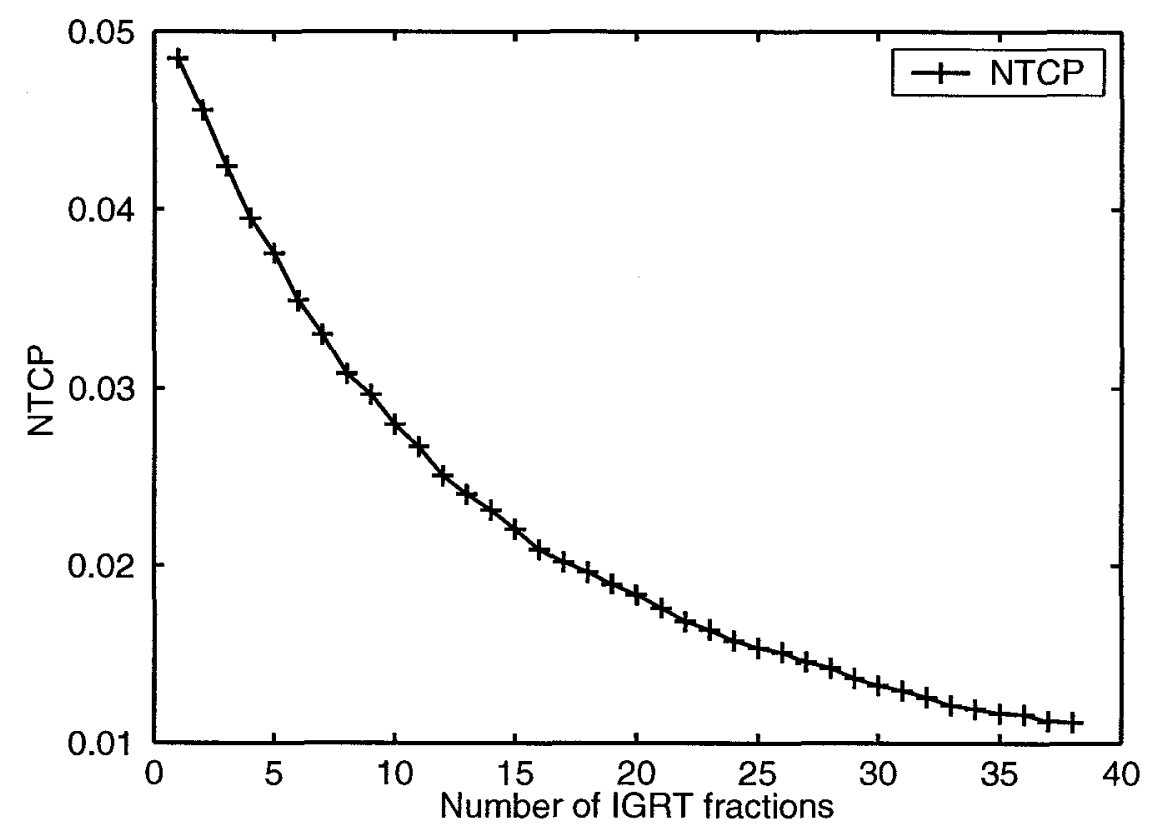

Figure 6.13 NTCP as a function of $N_{\text {IGRT }}$

The use of IGRT in prostate cancer treatments has two influences. Firstly, a large treatment margin is avoided because most of the geometric error can be corrected. Secondly, if IGRT is used as an adaptive technique, systematic error can be reduced. Increasing $n_{I G R T}$ means less systematic error and a smaller treatment margin and the combination of both can consistently reduce the normal tissue toxicity. This trend can be seen in Figure 6.13.

If the goal of IGRT is to escalate radiation dose to the tumour and subsequently achieve better tumour control probability while maintaining a consistent NTCP tolerance of 5\%, then our simulation indicates that $80 \mathrm{~Gy}$ can be safely delivered to the prostate CTV by performing 7 fractions with IGRT and 33 fractions with conventional technique. 


\subsection{Conclusions}

We have simulated the effects of geometric uncertainty on local tumour control for combinations of two margins and three treatment techniques. Several conclusions can be made from this simulation

First, the tumour control probability is sensitive to radiobiological parameters, in particular to the value of $\alpha / \beta$. If estimates of $\alpha / \beta=1.5$ for prostate cancer are true, hypofractionation schemes can improve therapeutic gains without increasing normal tissue toxicity. In other words, radiation dose can be delivered to the target with a few fractions and a large fraction size. The advantages of hypofractionation techniques are mainly from two aspects. They increase the therapeutic ratio to the tumour and reduce the patient's total treatment time, potentially saving radiotherapy resources. Since the $\alpha / \beta$ values are still under heated debated, the range for $\alpha / \beta$ values is relative wide $(0.8 \sim 10)^{[145,146,154-156]}$. If this variation is due to the difference from patient to patient, hypofractionation may be effective for patients with a lower $\alpha / \beta$ value $(\sim 1.5 \mathrm{~Gy})$ but not for those with a higher value ( $>8 \mathrm{~Gy}$ ).

Second, if the $\alpha / \beta$ value for prostate cancer is lower than for most cancers ( $>8 \mathrm{~Gy}$ ), but is not as low as 1.5 , there will be no therapeutic gain by using hypofractionation schemes. For instance, the value for prostate cancer is comparable to the late responding normal tissues ( $3.1 \mathrm{~Gy}$ ). In this case, the treatment margins must be optimized and the geometric uncertainty must be reasonably reduced to improve the TCP.

Third, systematic uncertainty has a greater impact on TCP than random uncertainty. With a conventional $10 \mathrm{~mm}$ margin, the effect of random uncertainty can be ignored. TCP could be improved by up to $3 \%$ if systematic error were fully removed.

IGRT can be employed efficiently using an adaptive technique. We have found that a treatment is optimized if 7 10 fractions of radiation are delivered with IGRT adaptive techniques. 


\section{CHAPTER 7 \\ General Conclusions and Scope for Future Work}

\subsection{General Conclusions}

\subsubsection{Conclusions from Geometric Uncertainty Measurements}

In this work, we have investigated the independent geometric errors associated with prostate cancer 3DCRT. The following conclusions are made based on data extracted from portal images acquired from 257 fractions of radiation delivery to 21 patients (14 prone patients with immobilization and 7 supine patients).

The global geometric error is the vector sum of all treatment preparation (simulation, planning) and delivery (positioning error, intra-fraction patient motion, inter- and intrafraction prostate movement within the pelvis) as well as linac geometric errors. The intrafraction organ motion is the position variation of the target within a treatment fraction. Global geometric error represents the maximum error one could expect in our practice and similarly intra-fraction organ motion represents the lower limit of the treatment error that we could be achieved. Based on our measurements, the global random geometric uncertainty was estimated to be $5.3 \mathrm{~mm}$ for all observed directions (LO, RO and SI). The intra-fraction organ motion was found to be within $1 \mathrm{~mm}$ for all observed directions, which is small with respect to overall geometric uncertainty and therefore the effect of intra-fraction prostate motion can be ignored for 3DCRT Note however, that this is significant when developing treatment margins for IGRT. 
Treatment preparation uncertainty or the distribution of individual systematic error describes the distribution of the geometric errors which arise prior to treatment delivery, while treatment delivery uncertainty describes the geometric uncertainty which occurs during radiation delivery. We have found that the treatment preparation uncertainty is generally larger than treatment delivery uncertainty.

Based on our analysis of global geometric error, we have found that the effectiveness of check films to mitigate against treatment deviations is very low (they can correct for only about $7 \%$ of all significant deviations) because they only correct for the random component of global geometric error such as setup error or internal target motion, but do not address the dominant systematic error.

The individual systematic error for a patient results from an inaccurate knowledge of the mean target position prior to the dose delivery. This can be effectively reduced if more independent treatment simulations are performed and averaged. Alternatively, in a technique known as adaptive radiation therapy, the mean target position is estimated over the first several treatment fractions, providing a more accurate estimate of mean target position. Subsequently a new isocentre or setup position is adopted for the remainder of treatment fractions the new data.

In this work we compared the difference in geometric uncertainties between supine and prone (with Hip-Fix immobilization) patient setup position. It was found that there is no significant difference in geometric uncertainty between the two techniques, however, a supine setup is superior to prone in terms of comfort and resource utilization.

\subsubsection{Target Delineation}

In this study, a gold standard prostate volume for the visible human male was established by a group of experts based on anatomical photographic images. The prostate volume for the same subject was also contoured on co-registered CT images by six different observers, each observer repeating the target delineation a total of 20 times. In total 120 
prostate delineations were obtained. Subsequently the CT and anatomical images were fused and following image registration measures of agreement between the two sets were calculated.

The radiation oncologist-delineated prostate volume on CT is on average $30 \%$ larger than the "true" gold standard volume. The agreement between CT delineated and true prostate volumes was used to describe the goodness of prostate definition. We have found that on average a CT prostate definition includes $84 \%( \pm 3 \%)$ of the true prostate volume, at the cost of including of $9.0 \mathrm{~cm}^{3}$ of normal tissues as part of the prostate definition.

Our results also indicated that there was a systematic difference in the geometric centre between physician-delineated prostate and true prostate, with a mean shift of $3.9 \mathrm{~mm}$ towards the patient's anterior direction. Our data suggests that radiation oncologists are more concerned with the accidental inclusion of rectal tissue than they are in missing prostate volume.. In contrast, they are likely to overextend the anterior boundary of the prostate to encompass normal tissue such as the bladder. This has also been verified by our gap analysis in which it was found that there was a missing gap of $2.8 \mathrm{~mm}$ in posterior direction and an overestimate (negative gap) of $5.8 \mathrm{~mm}$ in anterior direction between the physician-delineated and "true" prostate volume. The gap analysis indicated that posteriorly, although there is a significant systematic error, the physicians are very precise in their segmentation in this region, again suggesting that physicians are more worried about including rectum in their target volumes than they are in missing prostate volume. Conversely, in the anterior direction, radiation oncologists appear more worried about missing target volume than incorrectly including normal tissue (bladder) in the target volume.

Finally we observed that in general the inter-observer variation is larger than intraobservater variation for prostate volume definition, reflecting that the self-difference in target delineation is small compared to the group difference. The intra-observer variation may result from the radiation oncologists being inconsistent in their selection of window 
and level settings while the inter-observer variation is probably due to differences in the experience among the different observers.

In this work we have developed a tool we refer to as Probability Volume Histogram (PVH). Examining these PVHs, we found that a uniform margin of $5 \mathrm{~mm}$ around the CT delineated prostate volume can include $95 \%$ of the "true" prostate volume with a probability of $90 \%$.

\subsubsection{Effect of geometric uncertainty for local control and normal tissue complication}

We have simulated the effect of geometric uncertainty on local tumour control for combinations of two margins and three treatment techniques. Several conclusions were drawn from these simulations.

First, tumour control probability is sensitive to radiobiological parameters, in particular to the value of the $\alpha / \beta$ ratio. If prostate cancers do have a low value of $\alpha / \beta=1.5$, then hypo-fractionated schemes provide a biological advantage by improving the therapeutic gain for prostate cancer without increasing normal tissue toxicity. In other words, radiation dose can be delivered to the tumour target with a small number of large fractions. Under this scenario, there are two principal advantages of the hypofractionation technique: the increase in the therapeutic ratio to the tumour and a reduction in the patient's total treatment time which would likely, save on radiotherapy resources. The true value of the $\alpha / \beta$ ratio is still under heated debated with arguments ranging from 0.8 to 10 . Ultimately hypo-fractionation may be an effective treatment strategy for prostate cancer patients if a lower $\alpha / \beta$ value $(1.5 \mathrm{~Gy})$ can be demonstrated but would not be indicated if the $\alpha / \beta$ value turns to be a higher value ( $>8 \mathrm{~Gy}$ ).

If $\alpha / \beta$ for prostate cancer is found to be comparable to that of late responding normal tissues (3.1 Gy), our studies have shown that an appropriate treatment strategy is for the margin to be optimized and the geometric uncertainty reasonably reduced. The effect of 
geometric uncertainty and treatment margins were emphasized in our simulations. From this study, we found that both TCP and NTCP are sensitive to the choice of treatment margins rather than the random geometric uncertainty.

The treatment preparation uncertainty, sometimes referred to as the distribution of individual systematic error has a greater impact on TCP than random uncertainty. When a conventional $10 \mathrm{~mm}$ margin is used the effect of random uncertainty can be ignored even in the presence of the geometric error. It was also shown that the TCP can be improved by up to $3 \%$ if systematic error can be fully corrected.

\subsection{Scope for Future Work}

\subsubsection{Algorithm Application}

Although the objective of this thesis was to measure the independent geometric errors in the prostate cancer 3DCRT, much of the effort was directed to improving the quality of the portal images (contrast and distortion) acquired from Siemens Beamview T1 imager. The algorithms developed for this research could easily be applied to filmless linac QA. In a radiotherapy department, there are always intensive requirements to verify the radiation field shaped by MLC. This task is often accomplished with conventional films. An EPID is an ideal device for film less quantity assurance in radiotherapy practice, but has not been implemented in most centres. It was reported that by the end of 2002, only $5 \%$ of cancer centres have a filmless QA program based on an EPI. There are several barriers to the implementation of an EPID for routine QA: physicists are more familiar with QA using conventional films, and it will take time to complete the transformation from conventional methods to filmless. EPID image quality is still poor and cannot compete with film for overall quality although it has been improved significantly over the last few years. A lack of dedicated software for processing portal images might be the major problem limiting the EPID application in the QA program. 
As an example, the calibration of the MLC leaves with EPID devices is suggested as follows: For each gantry angle, the beam isocentre is determined using the Radon transform method and inter-leaf radiation leakage as described in Chapter 3. Both axis are so defined by two exposures acquired for collimator $0^{\circ}$ and $90^{\circ}$. Since the beam isocentre and leaf position along the solid jaw traveling direction is well defined, the edges for both the $\mathrm{X} 1$ and $\mathrm{X} 2$ banks can be obtained through local thresholding technique. The comparison of the measured leaf end position to the planned edges can provide the basis for an independent leaf calibration. The advantage of this approach is that both banks can be independently calibrated and the systematic error for each bank can be measured. It will be useful to integrate these algorithms into a routine linac QA program.

\subsubsection{Target repositioning}

The initial objective of this study was to investigate the feasibility of the prostate repositioning by using two orthogonal portal images prior to the treatment delivery. There are several barriers to online target positioning. Target repositioning requires algorithms for automatic seed localization. However, automatic seed detection is compromised by the poor image contrast and the small field sizes used for prostate cancer treatment. We found that in approximately $10 \%$ of all treatment fractions at least one seed is not in the treated field or is close to the field boundary, which makes it impossible to detect seeds in the image and to perform seed triangulation. In order to perform automatic target repositioning with two orthogonal images, we suggest that imager device should be upgraded to get reasonable image contrast for automatic seed detection. A relatively large portal should be used to obtain seed location prior to the target repositioning. Both may be further investigated for future application.

\subsubsection{Target delineation}

By taking advantage of the anatomical data from the VHP, we created a gold standard for our target delineation study. We measured the difference between CT delineated prostate 
volumes and the gold standard, and found a significant systematic difference between the CT delineated volume and the "true" prostate. It is important to note that this systematic error cannot be measured in a real cancer patient. Determining a systematic error is only possible because of the indexed photographic data set providing a "gold" standard. To our knowledge, all other clinical studies of target delineations have used the population average as the gold standard. It is worthwhile to note that the VHP data also includes a complete set of MRI images for the visible human male. A future study of MRI based target delineation may also provide valuable information on prostate target delineation, particularly in light of the fact that MRI is known to provide better soft tissue contrast than CT. We suggest that a study should be performed to investigate the delineation difference between the photographic anatomical images and the MRI images. This study will address the question as to whether the systematic error is physician related or modality related. If the systematic error between MRI delineated volume and "true" prostate volume is reduced, this will be valuable evidence to pursue MRI as the routine modality for prostate target delineation. If a systematic error exists between MRI delineated and the "true" target volume, it could conclude that the systematic error is due to the radiation oncologist's performance rather than the modality. The comparison of prostate delineation between MRI and photographic images will be a valuable supplement to our target delineation study. 


\section{References}

[1] Prostate enlargement : benign prostatic hyperplasia, National Kidney and Urologic Diseases Information Clearinghouse,NIH-06-3012, Bethesda, MD (2006)

[2] Cancer Statistics Canada, Canadian Cancer Society, Toronto (2004).

[3] Chen, L., Price, R. A., Jr., Nguyen, T. B., Wang, L., Li, J. S., Qin, L., Ding, M., Palacio, E., Ma, C. M., and Pollack, A., Dosimetric evaluation of MRI-based treatment planning for prostate cancer, Phys. Med. Biol., 49:5157-5170 (2004)

[4] Bradley, J., Thorstad, W. L., Mutic, S., Miller, T. R., Dehdashti, F., Siegel, B. A., Bosch, W., and Bertrand, R. J., Impact of FDG-PET on radiation therapy volume delineation in non-small-cell lung cancer, Int.J. Radiat. Oncol. Biol. Phys., 59:78-86 (2004)

[5] Sannazzari, G. L., Ragona, R., Ruo Redda, M. G., Giglioli, F. R., Isolato, G., and Guarneri, A., CT-MRI image fusion for delineation of volumes in threedimensional conformal radiation therapy in the treatment of localized prostate cancer, Br. J. Radiol., 75:603-607 (2002)

[6] Lu, W., Olivera, G. H., Chen, M. L., Reckwerdt, P. J., and Mackie, T. R., Accurate convolution/superposition for multi-resolution dose calculation using cumulative tabulated kernels, Phys. Med. Biol., 50:655-680 (2005)

[7] Bucci, M. K., Bevan, A., and Roach, M., III, Advances in Radiation Therapy: Conventional to 3D, to IMRT, to 4D, and Beyond, CA Cancer J. Clin., 55:117-134 (2005)

[8] Chauvet, I., Gaboriaud, G., Pontvert, D., Zefkili, S., Giraud, P., Rosenwald, J. C., and Cosset, J. M., Constraints and dosage for prostate cancer patients treated with conformal radiotherapy and intensity modulated radiation therapy, Cancer Radiother., 8:337-351 (2004)

[9] Almond, P. R., Biggs, P. J., Coursey, B. M., Hanson, W. F., Huq, M. S., Nath, R., and Rogers, D. W., AAPM's TG-51 protocol for clinical reference dosimetry of high-energy photon and electron beams, Med. Phys., 26:1847-1870 (1999)

[10] Nath, R., Biggs, P. J., Bova, F. J., Ling, C. C., Purdy, J. A., van de, Geijn J., and Weinhous, M. S., AAPM code of practice for radiotherapy accelerators: report of AAPM Radiation Therapy Task Group No. 45, Med. Phys., 21:1093-1121 (1994)

[11] Wu, J., Haycocks, T., Alasti, H., Ottewell, G., Middlemiss, N., Abdolell, M., Warde, P., Toi, A., and Catton, C., Positioning errors and prostate motion during 
conformal prostate radiotherapy using on-line isocentre set-up verification and implanted prostate markers, Radiother. Oncol., 61:127-133 (2001)

[12] Shimizu, S., Shirato, H., Kitamura, K., Shinohara, N., Harabayashi, T., Tsukamoto, T., Koyanagi, T., and Miyasaka, K., Use of an implanted marker and real-time tracking of the marker for the positioning of prostate and bladder cancers, Int. J. Radiat. Oncol. Biol. Phys., 48:1591-1597 (2000)

[13] Murphy, M. J., Fiducial-based targeting accuracy for external-beam radiotherapy, Med. Phys., 29:334-344 (2002)

[14] Kneebone, A., Gebski, V., Hogendoorn, N., and Turner, S., A randomized trial evaluating rigid immobilization for pelvic irradiation, Int. J. Radiat. Oncol. Biol. Phys., 56:1105-1111 (2003)

[15] Baumert, B. G., Zagralioglu, O., Davis, J. B., Reiner, B., Luetolf, U. M., and Ciernik, I. F., The use of a leg holder immobilisation device in 3D-conformal radiation therapy of prostate cancer, Radiother. Oncol., 65:47-52 (2002)

[16] Girouard, L. M., Pouliot, J., Maldague, X., and Zaccarin, A., Automatic setup deviation measurements with electronic portal images for pelvic fields, Med. Phys., 25:1180-1185 (1998)

[17] Hunt, M. A., Schultheiss, T. E., Desobry, G. E., Hakki, M., and Hanks, G. E., An evaluation of setup uncertainties for patients treated to pelvic sites, Int. J. Radiat. Oncol. Biol. Phys., 32:227-233 (1995)

[18] Fielding, A. L., Evans, P. M., and Clark, C. H., The use of electronic portal imaging to verify patient position during intensity-modulated radiotherapy delivered by the dynamic MLC technique, Int. J. Radiat. Oncol. Biol. Phys., 54:1225-1234 (2002)

[19] ICRU report 50 prescribing, recording, and reporting photon beam therapy, International Commission on Radiation Units and Measurements, Bethesda, Maryland (1993).

[20] Wambersie, Andre, ICRU report 62 prescribing, recording and reporting photon beam therapy (supplement to ICRU report 50), Bethesda, Maryland (1999).

[21] Ackerman, M. J., Spitzer, V. M., Scherzinger, A. L., and Whitlock, D. G., The Visible Human data set: an image resource for anatomical visualization, Med info., 8 Pt 2:1195-1198 (1995)

[22] Faiz M.Khan, the physics of radiation therapy, $3^{\text {rd }}$ edition, Lippincott Williams \& Wilkins, Baltimore, Maryland (2003)

[23] Meijer, G. J., Rasch, C., Remeijer, P., and Lebesque, J. V., Three-dimensional analysis of delineation errors, setup errors, and organ motion during radiotherapy of bladder cancer, Int. J. Radiat. Oncol. Biol. Phys., 55:1277-1287 (2003) 
[24] Remeijer, P., Rasch, C., Lebesque, J. V., and van Herk, M., A general methodology for three-dimensional analysis of variation in target volume delineation, Med. Phys., 26:931-940 (1999)

[25] Leunens, G., Menten, J., Weltens, C., Verstraete, J., and van der, Schueren E., Quality assessment of medical decision making in radiation oncology: variability in target volume delineation for brain tumours, Radiother.Oncol., 29:169-175 (1993)

[26] Rasch, C., Barillot, I., Remeijer, P., Touw, A., van Herk, M., and Lebesque, J. V., Definition of the prostate in CT and MRI: a multi-observer study, Int. J. Radiat. Oncol. Biol. Phys., 43:57-66 (1999)

[27] Fiorino, C., Reni, M., Bolognesi, A., Cattaneo, G. M., and Calandrino, R., Intraand inter-observer variability in contouring prostate and seminal vesicles: implications for conformal treatment planning, Radiother.Oncol., 47:285-292 (1998)

[28] Hurkmans, C. W., Borger, J. H., Pieters, B. R., Russell, N. S., Jansen, E. P., and Mijnheer, B. J., Variability in target volume delineation on CT scans of the breast, Int. J. Radiat. Oncol. Biol. Phys., 50:1366-1372 (2001)

[29] Kestin, L., Goldstein, N., Vicini, F., Yan, D., Korman, H., and Martinez, A., Treatment of prostate cancer with radiotherapy: should the entire seminal vesicles be included in the clinical target volume?, Int. J. Radiat. Oncol. Biol. Phys., 54:686697 (2002)

[30] Logue, J. P., Sharrock, C. L., Cowan, R. A., Read, G., Marrs, J., and Mott, D., Clinical variability of target volume description in conformal radiotherapy planning, Int. J. Radiat. Oncol. Biol. Phys., 41:929-931 (1998)

[31] Saarnak, A. E., Boersma, M., van Bunningen, B. N., Wolterink, R., and Steggerda, M. J., Inter-observer variation in delineation of bladder and rectum contours for brachytherapy of cervical cancer, Radiother. Oncol., 56:37-42 (2000)

[32] Weiss, E., Richter, S., Krauss, T., Metzelthin, S. I., Hille, A., Pradier, O., Siekmeyer, B., Vorwerk, H., and Hess, C. F., Conformal radiotherapy planning of cervix carcinoma: differences in the delineation of the clinical target volume. A comparison between gynaecologic and radiation oncologists, Radiother. Oncol., 67:87-95 (2003)

[33] Cazzaniga, L. F., Marinoni, M. A., Bossi, A., Bianchi, E., Cagna, E., Cosentino, D., Scandolaro, L., Valli, M., and Frigerio, M., Interphysician variability in defining the planning target volume in the irradiation of prostate and seminal vesicles, Radiother. Oncol., 47:293-296 (1998)

[34] Hurkmans, C. W., Remeijer, P., Lebesque, J. V., and Mijnheer, B. J., Set-up verification using portal imaging; review of current clinical practice, Radiother. Oncol., 58:105-120 (2001) 
[35] Yan, D., Lockman, D., Brabbins, D., Tyburski, L., and Martinez, A., An off-line strategy for constructing a patient-specific planning target volume in adaptive treatment process for prostate cancer, Int. J. Radiat. Oncol. Biol. Phys., 48:289-302 (2000)

[36] Yan, D. and Lockman, D., Organ/patient geometric variation in external beam radiotherapy and its effects, Med. Phys., 28:593-602 (2001)

[37] Booth, J, Modelling the impact of treatment uncertainties in radiotherapy, Ph.D thesis, University of Adelaide, Australia (2002).

[38] el Gayed, A. A., Bel, A., Vijlbrief, R., Bartelink, H., and Lebesque, J. V., Time trend of patient setup deviations during pelvic irradiation using electronic portal imaging, Radiother. Oncol., 26:162-171 (1993)

[39] Bijhold, J., Gilhuijs, K. G., and van Herk, M., Automatic verification of radiation field shape using digital portal images, Med. Phys., 19:1007-1014 (1992)

[40] Bayley, A. J., Catton, C. N., Haycocks, T., Kelly, V., Alasti, H., Bristow, R., Catton, P., Crook, J., Gospodarowicz, M. K., McLean, M., Milosevic, M., and Warde, P., A randomized trial of supine vs. prone positioning in patients undergoing escalated dose conformal radiotherapy for prostate cancer, Radiother. Oncol., 70:37-44 (2004)

[41] Alasti, H., Petric, M. P., Catton, C. N., and Warde, P. R., Portal imaging for evaluation of daily on-line setup errors and off-line organ motion during conformal irradiation of carcinoma of the prostate, Int. J. Radiat. Oncol. Biol. Phys., 49:869$884(2001)$

[42] Creutzberg, C. L., Althof, V. G., de Hoog, M. D., Visser, A. G., Huizenga, H., Wijnmaalen, A., and Levendag, P. C., A quality control study of the accuracy of patient positioning in irradiation of pelvic fields, Int. J. Radiat. Oncol. Biol. Phys., 34:697-708 (1996)

[43] Mackie, T. R., Kapatoes, J., Ruchala, K., Lu, W., Wu, C., Olivera, G., Forrest, L., Tome, W., Welsh, J., Jeraj, R., Harari, P., Reckwerdt, P., Paliwal, B., Ritter, M., Keller, H., Fowler, J., and Mehta, M., Image guidance for precise conformal radiotherapy, Int. J. Radiat. Oncol. Biol. Phys., 56:89-105 (2003)

[44] Huang, E., Dong, L., Chandra, A., Kuban, D. A., Rosen, I. I., Evans, A., and Pollack, A., Intrafraction prostate motion during IMRT for prostate cancer, Int. J. Radiat. Oncol. Biol. Phys., 53:261-268 (2002)

[45] Crook, J. M., Raymond, Y., Salhani, D., Yang, H., and Esche, B., Prostate motion during standard radiotherapy as assessed by fiducial markers, Radiother. Oncol., $37: 35-42(1995)$ 
[46] Mageras, G. S. and Yorke, E., Deep inspiration breath hold and respiratory gating strategies for reducing organ motion in radiation treatment, Semin.Radiat.Oncol., 14:65-75 (2004)

[47] Remeijer, P., Geerlof, E., Ploeger, L., Gilhuijs, K., van Herk, M., and Lebesque, J. V., 3-D portal image analysis in clinical practice: an evaluation of 2-D and 3-D analysis techniques as applied to 30 prostate cancer patients, Int. J. Radiat. Oncol. Biol. Phys., 46:1281-1290 (2000)

[48] Mageras, G. S., Fuks, Z., Leibel, S. A., Ling, C. C., Zelefsky, M. J., Kooy, H. M., van Herk, M., and Kutcher, G. J., Computerized design of target margins for treatment uncertainties in conformal radiotherapy, Int. J. Radiat. Oncol. Biol. Phys., 43:437-445 (1999)

[49] van Herk, M., Gilhuijs, K. G., de Munck, J., and Touw, A., Effect of image artifacts, organ motion, and poor segmentation on the reliability and accuracy of three-dimensional chamfer matching, Comput. Aided Surg., 2:346-355 (1997)

[50] Bel, A., van Herk, M., and Lebesque, J. V., Target margins for random geometrical treatment uncertainties in conformal radiotherapy, Med. Phys., 23:1537-1545 (1996)

[51] van Herk, M., Bruce, A., Kroes, A. P., Shouman, T., Touw, A., and Lebesque, J. V., Quantification of organ motion during conformal radiotherapy of the prostate by three dimensional image registration, Int. J. Radiat. Oncol. Biol. Phys., 33:13111320 (1995)

[52] Bijhold, J., van Herk, M., Vijlbrief, R., and Lebesque, J. V., Fast evaluation of patient set-up during radiotherapy by aligning features in portal and simulator images, Phys.Med.Biol., 36:1665-1679 (1991)

[53] Jones, B., Aird, E., Colyer, H., Dobbs, J., Harris, R., Hoskin, P., McKenzie, A., and West, C., United Kingdom Radiation Oncology 1 Conference (UKRO 1): accuracy and uncertainty in radiotherapy, Br.J.Radiol., 75:297-306 (2002)

[54] Bel, A., van Herk, M., Bartelink, H., and Lebesque, J. V., A verification procedure to improve patient set-up accuracy using portal images, Radiother. Oncol., 29:253$260(1993)$

[55] Bijhold, J., Gilhuijs, K. G., van Herk, M., and Meertens, H., Radiation field edge detection in portal images, Phys.Med.Biol., 36:1705-1710 (1991)

[56] Bijhold, J., Gilhuijs, K. G., and van Herk, M., Automatic verification of radiation field shape using digital portal images, Med. Phys., 19:1007-1014 (1992)

[57] Bortfeld, T., van Herk, M., and Jiang, S. B., When should systematic patient positioning errors in radiotherapy be corrected?, Phys. Med. Biol., 47:N297-N302 (2002) 
[58] Cho, B. C., van Herk, M., Mijnheer, B. J., and Bartelink, H., The effect of set-up uncertainties, contour changes, and tissue inhomogeneities on target dose-volume histograms, Med. Phys., 29:2305-2318 (2002)

[59] Deurloo, K. E., Steenbakkers, R. J., Zijp, L. J., de Bois, J. A., Nowak, P. J., Rasch, C. R., and van Herk, M., Quantification of shape variation of prostate and seminal vesicles during external beam radiotherapy, Int. J. Radiat. Oncol. Biol. Phys., 61:228-238 (2005)

[60] Hoogeman, M. S., van Herk, M., de Bois, J., and Lebesque, J. V., Strategies to reduce the systematic error due to tumor and rectum motion in radiotherapy of prostate cancer, Radiother. Oncol., 74:177-185 (2005)

[61] Lotz, H. T., Remeijer, P., van Herk, M., Lebesque, J. V., de Bois, J. A., Zijp, L. J., and Moonen, L. M., A model to predict bladder shapes from changes in bladder and rectal filling, Med. Phys., 31:1415-1423 (2004)

[62] McKenzie, A., van Herk, M., and Mijnheer, B., Margins for geometric uncertainty around organs at risk in radiotherapy, Radiother. Oncol., 62:299-307 (2002)

[63] McKenzie, A. L., van Herk, M., and Mijnheer, B., The width of margins in radiotherapy treatment plans, Phys. Med. Biol., 45:3331-3342 (2000)

[64] Remeijer, P., Rasch, C., Lebesque, J. V., and van Herk, M., Margins for translational and rotational uncertainties: a probability-based approach, Int. J. Radiat. Oncol. Biol. Phys., 53:464-474 (2002)

[65] Zelefsky, M. J., Crean, D., Mageras, G. S., Lyass, O., Happersett, L., Ling, C. C., Leibel, S. A., Fuks, Z., Bull, S., Kooy, H. M., van Herk, M., and Kutcher, G. J., Quantification and predictors of prostate position variability in 50 patients evaluated with multiple CT scans during conformal radiotherapy, Radiother. Oncol., 50:225-234 (1999)

[66] Langen, K. M. and Jones, D. T., Organ motion and its management, Int. J. Radiat. Oncol. Biol. Phys., 50:265-278 (2001)

[67] Yan, D., Ziaja, E., Jaffray, D., Wong, J., Brabbins, D., Vicini, F., and Martinez, A., The use of adaptive radiation therapy to reduce setup error: a prospective clinical study, Int. J. Radiat. Oncol. Biol. Phys., 41:715-720 (1998)

[68] van Herk, M., Errors and margins in radiotherapy, Semin. Radiat. Oncol., 14:52-64 (2004)

[69] Malone, S., Crook, J. M., Kendal, W. S., and Szanto, J., Respiratory-induced prostate motion: quantification and characterization, Int. J. Radiat. Oncol. Biol. Phys., 48:105-109 (2000) 
[70] Goitein, M., Organ and tumor motion: an overview, Semin. Radiat. Oncol., 14:2-9 (2004)

[71] Ten Haken, R. K., Forman, J. D., Heimburger, D. K., Gerhardsson, A., McShan, D. L., Perez-Tamayo, C., Schoeppel, S. L., and Lichter, A. S., Treatment planning issues related to prostate movement in response to differential filling of the rectum and bladder, Int. J. Radiat. Oncol. Biol. Phys., 20:1317-1324 (1991)

[72] Schild, S. E., Casale, H. E., and Bellefontaine, L. P., Movements of the prostate due to rectal and bladder distension: implications for radiotherapy, Med.Dosim., 18:1315 (1993)

[73] Dehnad, H., Nederveen, A. J., van der Heide, U. A., van Moorselaar, R. J., Hofman, P., and Lagendijk, J. J., Clinical feasibility study for the use of implanted gold seeds in the prostate as reliable positioning markers during megavoltage irradiation, Radiother. Oncol., 67:295-302 (2003)

[74] Aubry, J. F., Beaulieu, L., Girouard, L. M., Aubin, S., Tremblay, D., Laverdiere, J., and Vigneault, E., Measurements of intrafraction motion and interfraction and intrafraction rotation of prostate by three-dimensional analysis of daily portal imaging with radiopaque markers, Int. J. Radiat. Oncol. Biol. Phys., 60:30-39 (2004)

[75] Balter, J. M., Lam, K. L., Sandler, H. M., Littles, J. F., Bree, R. L., and Ten Haken, R. K., Automated localization of the prostate at the time of treatment using implanted radiopaque markers: technical feasibility, Int. J. Radiat. Oncol. Biol. Phys., 33:1281-1286 (1995)

[76] Buck, D., Alber, M., and Nusslin, F., Potential and limitations of the automatic detection of fiducial markers using an amorphous silicon flat-panel imager, Phys. Med. Biol., 48:763-774 (2003)

[77] Vigneault, E., Pouliot, J., Laverdiere, J., Roy, J., and Dorion, M., Electronic portal imaging device detection of radioopaque markers for the evaluation of prostate position during megavoltage irradiation: a clinical study, Int. J. Radiat. Oncol. Biol. Phys., 37:205-212 (1997)

[78] Malone, S., Donker, R., Dahrouge, S., Eapen, L., Aref, I., Perry, G., and Szanto, J., Treatment planning aids in prostate cancer: friend or foe?, Int. J. Radiat. Oncol. Biol. Phys., 51:49-55 (2001)

[79] Beavis, A. W., Is tomotherapy the future of IMRT?, Br.J.Radiol., 77:285-295 (2004)

[80] Orton, N. P. and Tome, W. A., The impact of daily shifts on prostate IMRT dose distributions, Med. Phys., 31:2845-2848 (2004) 
[81] Webb, S. and Nahum, A. E., A model for calculating tumour control probability in radiotherapy including the effects of inhomogeneous distributions of dose and clonogenic cell density, Phys. Med. Biol., 38:653-666 (1993)

[82] van Herk, M., Remeijer, P., and Lebesque, J. V., Inclusion of geometric uncertainties in treatment plan evaluation, Int. J. Radiat. Oncol. Biol. Phys., 52:1407-1422 (2002)

[83] van Herk, M., Witte, M., van der, Geer J., Schneider, C., and Lebesque, J. V., Biologic and physical fractionation effects of random geometric errors, Int. J. Radiat. Oncol. Biol. Phys., 57:1460-1471 (2003)

[84] Stroom, J. C., de Boer, H. C., Huizenga, H., and Visser, A. G., Inclusion of geometrical uncertainties in radiotherapy treatment planning by means of coverage probability, Int. J. Radiat. Oncol. Biol. Phys., 43:905-919 (1999)

[85] Stroom, J. C. and Heijmen, B. J., Geometrical uncertainties, radiotherapy planning margins, and the ICRU-62 report, Radiother. Oncol., 64:75-83 (2002)

[86] Song, W. and Dunscombe, P., EUD-based margin selection in the presence of setup uncertainties, Med. Phys., 31:849-859 (2004)

[87] Tubic, D., Zaccarin, A., Pouliot, J., and Beaulieu, L., Automated seed detection and three-dimensional reconstruction. I. Seed localization from fluoroscopic images or radiographs, Med. Phys., 28:2265-2271 (2001)

[88] Paskalev, K., Ma, C. M., Jacob, R., Price, R., McNeeley, S., Wang, L., Movsas, B., and Pollack, A., Daily target localization for prostate patients based on 3D image correlation, Phys. Med. Biol., 49:931-939 (2004)

[89] Pang, G., Beachey, D. J., O'Brien, P. F., and Rowlands, J. A., Imaging of 1.0-mmdiameter radiopaque markers with megavoltage $\mathrm{X}$-rays: an improved online imaging system, Int. J. Radiat. Oncol. Biol. Phys., 52:532-537 (2002)

[90] Antolak, J. A., Rosen, I. I., Childress, C. H., Zagars, G. K., and Pollack, A., Prostate target volume variations during a course of radiotherapy, Int. J. Radiat. Oncol. Biol. Phys., 42:661-672 (1998)

[91] Zellars, R. C., Roberson, P. L., Strawderman, M., Zhang, D., Sandler, H. M., Ten Haken, R. K., Osher, D., and McLaughlin, P. W., Prostate position late in the course of external beam therapy: patterns and predictors, Int. J. Radiat. Oncol. Biol. Phys., 47:655-660 (2000)

[92] Boyer, A. L., Antonuk, L., Fenster, A., van Herk, M., Meertens, H., Munro, P., Reinstein, L. E., and Wong, J., A review of electronic portal imaging devices (EPIDs), Med. Phys., 19:1-16 (1992) 
[93] Lattanzi, J., McNeely, S., Hanlon, A., Das, I., Schultheiss, T. E., and Hanks, G. E., Daily CT localization for correcting portal errors in the treatment of prostate cancer, Int. J. Radiat. Oncol. Biol. Phys., 41:1079-1086 (1998)

[94] Fung, A. Y., Grimm, S. Y., Wong, J. R., and Uematsu, M., Computed tomography localization of radiation treatment delivery versus conventional localization with bony landmarks, J. Appl. Clin. Med. Phys., 4:112-119 (2003)

[95] Uematsu, M., Fukui, T., Shioda, A., Tokumitsu, H., Takai, K., Kojima, T., Asai, Y., and Kusano, S., A dual computed tomography linear accelerator unit for stereotactic radiation therapy: a new approach without cranially fixated stereotactic frames, Int. J. Radiat. Oncol. Biol. Phys., 35:587-592 (1996)

[96] Boyer, A. L., Antonuk, L., Fenster, A., van Herk, M., Meertens, H., Munro, P., Reinstein, L. E., and Wong, J., A review of electronic portal imaging devices (EPIDs), Med. Phys., 19:1-16 (1992)

[97] Jaffray, D. A., Siewerdsen, J. H., Wong, J. W., and Martinez, A. A., Flat-panel cone-beam computed tomography for image-guided radiation therapy, Int. J. Radiat. Onciol. Biol. Phys., 53:1337-1349 (2002)

[98] Nederveen, A., Lagendijk, J., and Hofman, P., Detection of fiducial gold markers for automatic on-line megavoltage position verification using a marker extraction kernel (MEK), Int. J. Radiat. Oncol. Biol. Phys., 47:1435-1442 (2000)

[99] Craig, T., Moiseenko, V., Battista, J., and Van Dyk, J., The impact of geometric uncertainty on hypofractionated external beam radiation therapy of prostate cancer, Int. J. Radiat. Oncol. Biol. Phys., 57:833-842 (2003)

[100]Kutcher, G. J., Mageras, G. S., and Liebel, S. A., Control, Correction, and Modeling of Setup Errors and Organ Motion, Semin. Radiat. Oncol., 5:134-145 (1995)

[101] Antolak, J. A. and Rosen, I. I., Planning target volumes for radiotherapy: how much margin is needed?, Int. J. Radiat. Oncol. Biol. Phys., 44:1165-1170 (1999)

[102] Goitein, M., Calculation of the uncertainty in the dose delivered during radiation therapy, Med. Phys., 12:608-612 (1985)

[103] van Herk, M., Remeijer, P., Rasch, C., and Lebesque, J. V., The probability of correct target dosage: dose-population histograms for deriving treatment margins in radiotherapy, Int. J. Radiat. Oncol. Biol. Phys., 47:1121-1135 (2000)

[104]Brahme, A., Optimization of radiation therapy and the development of multi-leaf collimation, Int. J. Radiat. Oncol. Biol. Phys., 25:373-375 (1993)

[105]Webb, S., Intensity-Modulated Radiation Therapy, Institute of Physics Publishing, Bristol (2001) 
[106] Intensity-modulated radiotherapy: current status and issues of interest, Int. J. Radiat. Oncol. Biol. Phys., 51:880-914 (2001)

[107]Bortfeld, T., Jiang, S. B., and Rietzel, E., Effects of motion on the total dose distribution, Semin.Radiat.Oncol., 14:41-51 (2004)

[108]Keall, P. J., Todor, A. D., Vedam, S. S., Bartee, C. L., Siebers, J. V., Kini, V. R., and Mohan, R., On the use of EPID-based implanted marker tracking for 4D radiotherapy, Med. Phys., 31:3492-3499 (2004)

[109]Mackie, T. R., Holmes, T., Swerdloff, S., Reckwerdt, P., Deasy, J. O., Yang, J., Paliwal, B., and Kinsella, T., Tomotherapy: a new concept for the delivery of dynamic conformal radiotherapy, Med. Phys., 20:1709-1719 (1993)

[110]Balog, J., Lucas, D., DeSouza, C., and Crilly, R., Helical tomotherapy radiation leakage and shielding considerations, Med. Phys., 32:710-719 (2005)

[111] Keall, P., 4-dimensional computed tomography imaging and treatment planning, Semin.Radiat.Oncol., 14:81-90 (2004)

[112]Shirato, H., Shimizu, S., Kunieda, T., Kitamura, K., van Herk, M., Kagei, K., Nishioka, T., Hashimoto, S., Fujita, K., Aoyama, H., Tsuchiya, K., Kudo, K., and Miyasaka, K., Physical aspects of a real-time tumor-tracking system for gated radiotherapy, Int. J. Radiat. Oncol. Biol. Phys., 48:1187-1195 (2000)

[113]Serago, C. F., Chungbin, S. J., Buskirk, S. J., Ezzell, G. A., Collie, A. C., and Vora, S. A., Initial experience with ultrasound localization for positioning prostate cancer patients for external beam radiotherapy, Int. J. Radiat. Oncol. Biol. Phys., 53:1130$1138(2002)$

[114]Minohara, S., Kanai, T., Endo, M., Noda, K., and Kanazawa, M., Respiratory gated irradiation system for heavy-ion radiotherapy, Int. J. Radiat. Oncol. Biol. Phys., 47:1097-1103 (2000)

[115]Tada, T., Hosono, M., Fujioka, T., Fukuda, H., Nakajima, T., and Inoue, Y., Monitoring of respiratory movement of the diaphragm for gated radiotherapy, Radiat.Med., 23:10-13 (2005)

[116] Van Dyk J., the modern Technology of Radiation Oncology: A Compendium for medical physicists and radiation oncologists, medical physics publishing, Madison, WI (1999).

[117]Herman, M. G., Balter, J. M., Jaffray, D. A., McGee, K. P., Munro, P., Shalev, S., van Herk, M., and Wong, J. W., Clinical use of electronic portal imaging: report of AAPM Radiation Therapy Committee Task Group 58, Med. Phys., 28:712-737 (2001)

[118]Hubbell, J and S.M.Seltzer, Ionizing Radiation Division, Physics Laboratory 
National Institute of Standards and Technology. NISTIR 5632, Gaithersburgy, MD (1996).

[119] Antonuk, L. E., Electronic portal imaging devices: a review and historical perspective of contemporary technologies and research, Phys.Med.Biol., 47:R31R65 (2002)

[120] Gonzalez and Woods, Digital Image Processing, 2nd edition, Prentice Hall, (2002)

[121]Ronald N.Bracewell, The Fourier Transform and Its Applications, McGraw-Hill, New York, NY (1986)

[122]Dong, L. and Boyer, A. L., A portal image alignment and patient setup verification procedure using moments and correlation techniques, Phys. Med. Biol. 41:697-723 (1996)

[123]Winkler, P., Bergmann, H., Stuecklschweiger, G., and Guss, H., Introducing a system for automated control of rotation axes, collimator and laser adjustment for a medical linear accelerator, Phys.Med.Biol., 48:1123-1132 (2003)

[124]S.Zucker, Cross-correlation and maximum-likelihood analysis: a new approach to combining cross-correlation functions, Monthly Notices of the Royal Astronomical Society 342:1291-1298 (2003)

[125]Toft, Peter, The Radon Transform - Theory and Implementation, PhD thesis. Denmark Technology University, Denmark (1996).

[126]Khamene, A., Bloch, P., Wein, W., Svatos, M., and Sauer, F., Automatic registration of portal images and volumetric CT for patient positioning in radiation therapy, Med. Image Anal., 2005)

[127]Fraass, B., Doppke, K., Hunt, M., Kutcher, G., Starkschall, G., Stern, R., and Van Dyke, J., American Association of Physicists in Medicine Radiation Therapy Committee Task Group 53: quality assurance for clinical radiotherapy treatment planning, Med. Phys., 25:1773-1829 (1998)

[128]Gronenschild, E., Correction for geometric image distortion in the x-ray imaging chain: local technique versus global technique, Med. Phys., 26:2602-2616 (1999)

[129]Urie, M. M., Goitein, M., Doppke, K., Kutcher, J. G., LoSasso, T., Mohan, R., Munzenrider, J. E., Sontag, M., and Wong, J. W., The role of uncertainty analysis in treatment planning, Int. J. Radiat. Oncol. Biol. Phys., 21:91-107 (1991)

[130]Bayouth, J. E., Wendt, D., and Morrill, S. M., MLC quality assurance techniques for IMRT applications, Med. Phys., 30:743-750 (2003) 
[131]Tai, P., Van Dyk, J., Yu, E., Battista, J., Stitt, L., and Coad, T., Variability of target volume delineation in cervical esophageal cancer, Int. J. Radiat. Oncol. Biol. Phys., 42:277-288 (1998)

[132]Weltens, C., Menten, J., Feron, M., Bellon, E., Demaerel, P., Maes, F., Van den, Bogaert W., and van der, Schueren E., Interobserver variations in gross tumor volume delineation of brain tumors on computed tomography and impact of magnetic resonance imaging, Radiother. Oncol., 60:49-59 (2001)

[133] Wilt, T. J., Uncertainty in prostate cancer care: the physician's role in clearing the confusion, JAMA, 283:3258-3260 (2000)

[134]Rasch, C., Remeijer, P., Koper, P. C., Meijer, G. J., Stroom, J. C., van Herk, M., and Lebesque, J. V., Comparison of prostate cancer treatment in two institutions: a quality control study, Int. J. Radiat. Oncol. Biol. Phys., 45:1055-1062 (1999)

[135]Hanks, G. E., Hanlon, A. L., Epstein, B., and Horwitz, E. M., Dose response in prostate cancer with 8-12 years' follow-up, Int. J. Radiat. Oncol. Biol. Phys., $54: 427-435$ (2002)

[136] Pollack, A., prostate cancer radiation dose response: results of the M. D> Anderson phase III randomized trial, Urologic Oncology 21:306-316 (2003)

[137]Zelefsky, $M$ and Steven A.Leibel, Paul Gaundin, Dose escalation with threedimentional conformal radiation therapy affects the outcome in prostate cancer, Int. J. Radiat. Oncol. Biol. Phys. 41:491-500 (2004)

[138]Padhani, A. R., Khoo, V. S., Suckling, J., Husband, J. E., Leach, M. O., and Dearnaley, D. P., Evaluating the effect of rectal distension and rectal movement on prostate gland position using cine MRI, Int. J. Radiat. Oncol. Biol. Phys., 44:525533 (1999)

[139] Malone, S., Szanto, J., Perry, G., Gerig, L., Manion, S., Dahrouge, S., and Crook, J., A prospective comparison of three systems of patient immobilization for prostate radiotherapy, Int. J. Radiat. Oncol. Biol. Phys., 48:657-665 (2000)

[140]Hall, Eric, Radiobiology for the Radiologist, Fourth,edition J. B. Lippincott Company, Philadelphia, PA(1993)

[141]Chadwick, K. H. and Leenhouts, H. P., A molecular theory of cell survival, Phys.Med.Biol., 18:78-87 (1973)

[142]Song, W., Schaly, B., Bauman, G., Battista, J., and Van Dyk, J., Image-guided adaptive radiation therapy (IGART): Radiobiological and dose escalation considerations for localized carcinoma of the prostate, Med. Phys., 32:2193-2203 (2005) 
[143] Sachs, R. K., Hahnfeld, P., and Brenner, D. J., The link between low-LET doseresponse relations and the underlying kinetics of damage production/repair/misrepair, Int.J.Radiat.Biol., 72:351-374 (1997)

[144]Wang, J. Z., Li, X. A., D'Souza, W. D., and Stewart, R. D., Impact of prolonged fraction delivery times on tumor control: a note of caution for intensity-modulated radiation therapy (IMRT), Int. J. Radiat. Oncol. Biol. Phys., 57:543-552 (2003)

[145]Brenner, D. J. and Hall, E. J., Fractionation and protraction for radiotherapy of prostate carcinoma, Int. J. Radiat. Oncol. Biol. Phys., 43:1095-1101 (1999)

[146]Brenner, D. J., Martinez, A. A., Edmundson, G. K., Mitchell, C., Thames, H. D., and Armour, E. P., Direct evidence that prostate tumors show high sensitivity to fractionation (low alpha/beta ratio), similar to late-responding normal tissue, Int. J. Radiat. Oncol. Biol. Phys., 52:6-13 (2002)

[147]Barendsen, G. W., Dose fractionation, dose rate and iso-effect relationships for normal tissue responses, Int. J. Radiat. Oncol. Biol. Phys., 8:1981-1997 (1982)

[148]Fowler, J. F., The linear-quadratic formula and progress in fractionated radiotherapy, Br.J.Radiol., 62:679-694 (1989)

[149]Niemierko, A., Reporting and analyzing dose distributions: a concept of equivalent uniform dose, Med. Phys., 24:103-110 (1997)

[150]Song, W., Battista, J., and Van Dyk, J., Limitations of a convolution method for modeling geometric uncertainties in radiation therapy: the radiobiological dose-perfraction effect, Med. Phys., 31:3034-3045 (2004)

[151]Lyman, J. T., Complication probability as assessed from dose-volume histograms, Radiat.Res.Suppl, 8:S13-S19 (1985)

[152]Burman, C., Kutcher, G. J., Emami, B., and Goitein, M., Fitting of normal tissue tolerance data to an analytic function, Int. J. Radiat. Oncol. Biol. Phys., 21:123-135 (1991)

[153]Kutcher, G. J. and Burman, C., Calculation of complication probability factors for non-uniform normal tissue irradiation: the effective volume method, Int. J. Radiat. Oncol. Biol. Phys., 16:1623-1630 (1989)

[154]Nahum, A. E., Movsas, B., Horwitz, E. M., Stobbe, C. C., and Chapman, J. D., Incorporating clinical measurements of hypoxia into tumor local control modeling of prostate cancer: implications for the alpha/beta ratio, Int. J. Radiat. Oncol. Biol. Phys., 57:391-401 (2003) 
[155]Wang, J. Z., Guerrero, M., and Li, X. A., How low is the alpha/beta ratio for prostate cancer?, Int. J. Radiat. Oncol. Biol. Phys., 55:194-203 (2003)

[156]Brenner, D. J., Fractionation and late rectal toxicity, Int. J. Radiat. Oncol. Biol. Phys., 60:1013-1015 (2004)

[157]Lujan, A. E., Ten Haken, R. K., Larsen, E. W., and Balter, J. M., Quantization of setup uncertainties in 3-D dose calculations, Med. Phys., 26:2397-2402 (1999)

[158]Leong, J., Implementation of random positioning error in computerised radiation treatment planning systems as a result of fractionation, Phys.Med.Biol., 32:327-334 (1987)

[159]Craig, T., Battista, J., and Van Dyk, J., Limitations of a convolution method for modeling geometric uncertainties in radiation therapy. I. The effect of shift invariance, Med. Phys., 30:2001-2011 (2003)

[160]Lloyd-Davies, R. W., Collins, C. D., and Swan, A. V., Carcinoma of prostate treated by radical external beam radiotherapy using hypofractionation. Twenty-two years' experience (1962-1984), Urology, 36:107-111 (1990)

[161]Spanos, W. J., Jr., Wasserman, T., Meoz, R., Sala, J., Kong, J., and Stetz, J., Palliation of advanced pelvic malignant disease with large fraction pelvic radiation and misonidazole: final report of RTOG phase I/II study, Int. J. Radiat. Oncol. Biol. Phys., 13:1479-1482 (1987)

[162]Logue, J. P., Cowan, R. A., and Hendry, J. H., Hypofractionation for prostate cancer, Int. J. Radiat. Oncol. Biol. Phys., 49:1522-1523 (2001)

[163] Yan, D., Vicini, F., Wong, J., and Martinez, A., Adaptive radiation therapy, Phys. Med. Biol., 42:123-132 (1997) 